\title{
MEASUREMENT OF THE LEFT-RIGHT ASYMMETRY \\ IN $Z$ BOSON PRODUCTION \\ BY ELECTRON-POSITRON COLLISIONS ${ }^{*}$
}

\author{
Robert Dean Elia \\ Stanford Linear Accelerator Center \\ Stanford University \\ Stanford, CA 94309
}

April 1994

Prepared for the Department of Energy

under contract number DE-AC03-76SF00515

Printed in the United States of America. Available from the National Technical Information Service, U.S. Department of Commerce, 5285 Port Royal Road, Springfield, VA 22161.

${ }^{\star}$ Ph.D. thesis 


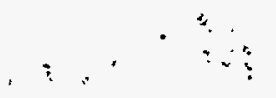




\section{DISCLAIMER}

This report was prepared as an account of work sponsored by an agency of the United States Government. Neither the United States Government nor any agency thereof, nor any of their employees, make any warranty, express or implied, or assumes any legal liability or responsibility for the accuracy, completeness, or usefulness of any information, apparatus, product, or process disclosed, or represents that its use would not infringe privately owned rights. Reference herein to any specific commercial product, process, or service by trade name, trademark, manufacturer, or otherwise does not necessarily constitute or imply its endorsement, recommendation, or favoring by the United States Government or any agency thereof. The views and opinions of authors expressed herein do not necessarily state or reflect those of the United States Government or any agency thereof. 


\section{DISCLAIMER}

Portions of this document may be illegible in electronic image products. Images are produced from the best available original document. 


\section{Abstract}

We present the first measurement of the left-right cross-section asymmetry $\left(A_{L R}\right)$ in $Z$ boson production observed at the SLAC Linear Collider. The left-right asymmetry provides a direct measurement of the $e^{-}-Z$ coupling and thus of the effective weak mixing angle; in addition, $A_{L R}$ is sensitive to the unknown top-quark and Higgs-boson masses. In 1992 the SLD detector recorded $10224 Z$ events produced by the collision of longitudinally polarized electrons with an unpolarized positron beam at a center-of-mass energy of $91.55 \mathrm{GeV}$. The average electron beam polarization during the run was $(22.4 \pm 0.6) \%$. We measure $A_{L R}$ to be $0.101 \pm 0.044$ (stat.) \pm 0.004 (syst.), which determines the effective weak mixing angle to be $\sin ^{2} \theta_{W}^{\text {eff }}=0.2377 \pm 0.0056$ (stat.) \pm 0.0005 (syst.). This measurement of $A_{L R}$ is in agreement with comparable measurements and is consistent with Standard-Model predictions for allowed top and Higgs masses. 



\section{Acknowledgements}

Hey-Bruce, you owe me \$20. Well, I can't believe it's over, but it's over, and if the next step is half as fun as this has been, I should have stayed here. Thanks go first to my adviser Morris Swartz, who knows more than anyone about anything of interest and plenty more besides. I consider myself fortunate to have learned from someone of his enormous intellect so early in my career. I also look forward to feeling less dumb in the future. Thanks also to Gary Feldman for adviserly duties in the Mark II era, and to his successor in B198, Charlie Prescott, for generous advice and guidance. I thank Marty Breidenbach for the use of his detector and for his words of wisdom. Working with Herb Steiner at LBL has been very rewarding, and I thank him for the experience. Special gratitude goes to Ken Moffeit, without whose tireless efforts and eternal optimism this whole project would still be SLAC-PROPOSAL-SLC-UPGRADE-01. Ken has been a true leader and source of inspiration to me, and his good nature and warm spirit have encouraged us all.

Of course, grad school wasn't all work. In fact, most of it was standing in the hall talking about bodily functions. Some of my more memorable discussions have been with Bruce Schumm, postdoc/mentor/storyteller/blues harpist extraordinaire. I couldn't have asked for more in a surrogate adviser, and Bruce couldn't have given more: knowledge, help, advice (physics and otherwise), extended jokes with disappointing punchlines, you name it. Working with Bruce has been a highlight of my grad school years, and I will miss him tremendously. To future jukes! Peter Rowson contributed his own inimitable brand of humor, as well as a boundless enthusiasm that was occasionally infectious. Peter took ample advantage of my open-door policy-I shudder to think of the physics that could have been accomplished in the countless hours we wasted. Mike "The Only One With Kids" Fero was the real conscience of the group, although I'm sure he'd rather be remembered as the surfer guitarist. I'll remember the sober veneer that always broke into self-deprecating laughter. Dave Calloway shared my keen interest in freak-watching, a hobby easily indulged at SLAC. Dave 
serves as living proof that physicists need not watch Star Trek and, in rare instances, can even possess a "personality", complete with "emotions" and "sensitivity". I am indebted to Mike Woods for his perseverence and dedication to the project, as well as his guidance, not to mention the softball coaching. Working with my fellow grad students Ross King and Amit Lath has been both a pleasure and a challenge. Needless to say, their efforts are reflected in this thesis; I hope I can return the favor, and I wish them both luck and speed. I am grateful to my former officemate Mina Petradza for her encouragement and help, and also to the current, Robin Erbacher, for the chuckles. In the Publications department, Sylvia MacBride worked heroically to provide the pictures for this thesis. Armin Wolff, Ed Tillmann, and Walter Kapica of the Cryo group have all been extremely helpful and kind over the years. I especially thank Group H's last secretary, June Hu, and her successor, Julie Rogers, for their professional excellence and friendship. I would like to thank the Petri ${ }^{\mathrm{TM}}$ Cookie company and Canteen Vending Service for the physical and spiritual sustenance their nourishing products have provided over the years.

My friends, both old and new, have somehow stuck around through this ordeal. Adrian Lee, my first and closest Stanford friend, ceaselessly tried against all odds to get me out of the house to enjoy myself. I truly appreciate it, along with all the support and companionship. Thanks also to Adrian and Michael Cohen for all the therapeutic jams. Jon Labs was a great, if troll-like, roommate for many years. Don Marr and Chris Silvey have to accept my apologies for the years of neglect and my thanks for being the best friends anyone could hope for. Ditto for Blake Edgar and Kevin Mayeda, my venerable ski buddies. How about Vermont next year? Special thanks go to Jennie Bishop for her wisdom, laughter, and friendship. I think I learned more from her than in all my classes.

My family has continued to be overwhelmingly supportive in every way. It is hard to express the debt of gratitude I owe them, since I owe them everything. I feel truly blessed. Speaking of blessed, the last three years with Elaine Sang have been wonderful. Her love and generosity are boundless and humbling. Thank you, Elaine, for everything.

And thanks again to everyone who has touched my life these past $7 \frac{1}{2}$ years. There were moments of pain and frustration, but there were times when everything felt just perfect.

This work is dedicated to my mother and father. 


\section{Contents}

Abstract

Acknowledgements $\quad$ v

1 The Left-Right Asymmetry 1

1.1 The Electroweak Standard Model . . . . . . . . . . . . . . . . . . . 1

1.1.1 The electroweak interaction . . . . . . . . . . . . . . 1

1.1.2 Higgs mechanism and boson masses $\ldots \ldots \ldots \ldots \ldots \ldots$

1.1.3 Electroweak parameters . . . . . . . . . . . . . . . 6

$1.2 \quad Z$ Production and Decay . . . . . . . . . . . . . . . 7

1.2.1 The electroweak neutral current $\ldots \ldots \ldots \ldots \ldots \ldots \ldots$

$1.2 .2 \quad Z$ cross section $\ldots \ldots \ldots \ldots \ldots \ldots \ldots \ldots \ldots \ldots$

1.3 Radiative Corrections to $Z$ Production . . . . . . . . . . . . . . . . 10

1.3.1 Initial-state radiation $\ldots \ldots \ldots \ldots \ldots \ldots \ldots$

1.3 .2 Virtual corrections . . . . . . . . . . . . . . 12

1.4 Electroweak Asymmetries . . . . . . . . . . . . . . . . . 17

1.4.1 Forward-backward asymmetry $\ldots \ldots \ldots \ldots \ldots \ldots \ldots$

$1.4 .2 \tau$-polarization asymmetry $\ldots \ldots \ldots \ldots \ldots \ldots \ldots \ldots$

1.5 The Left-Right Asymmetry . . . . . . . . . . . . . . . . . 20

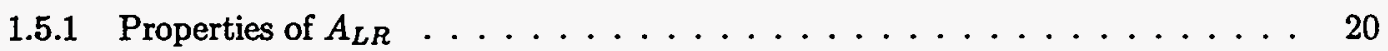

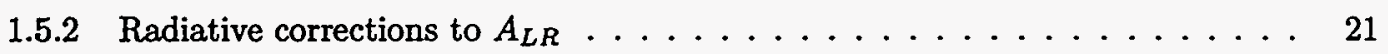

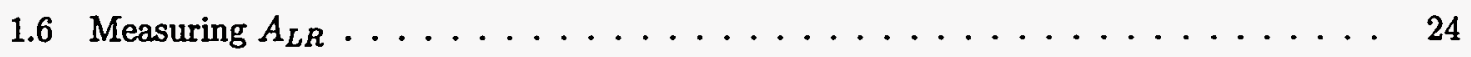

1.6.1 Error equation ...................... 24

1.6.2 Polarization measurement . . . . . . . . . . . . . . . 24 
2 Apparatus 27

2.1 The Polarized SLC . . . . . . . . . . . . . . . . . 27

2.1 .1 Polarized source $\ldots \ldots \ldots \ldots \ldots \ldots \ldots \ldots \ldots$

2.1 .2 Spin rotation $\ldots \ldots \ldots \ldots \ldots \ldots \ldots \ldots \ldots \ldots \ldots \ldots \ldots$

2.1.3 Linac Møller polarimeter . . . . . . . . . . . . . . 36

2.1 .4 Energy spectrometers $\ldots \ldots \ldots \ldots \ldots \ldots \ldots$

2.1.5 Extraction-line Møller polarimeter . . . . . . . . . . . . 38

2.2 The Compton Polarimeter . . . . . . . . . . . . . . . . . . 38

2.2 .1 Light source, transport, and monitoring . . . . . . . . . . . . . 39

2.2 .2 Čerenkov detector $\ldots \ldots \ldots \ldots \ldots \ldots \ldots \ldots \ldots$

2.2.3 Proportional tube detector $\ldots \ldots \ldots \ldots \ldots \ldots \ldots$

2.2 .4 Electronics and data acquisition $\ldots \ldots \ldots \ldots \ldots \ldots$

2.3 The SLD Detector $\ldots \ldots \ldots \ldots \ldots \ldots \ldots \ldots \ldots \ldots$

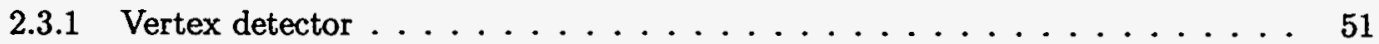

2.3 .2 Drift chambers . . . . . . . . . . . . . . . 51

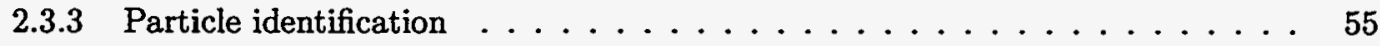

2.3 .4 Calorimetry ........................ 56

2.3.5 Luminosity monitoring/small-angle detection . . . . . . . . . . . 59

3 Polarization Measurement $\quad 61$

3.1 Compton Polarimetry . . . . . . . . . . . . . . . . 61

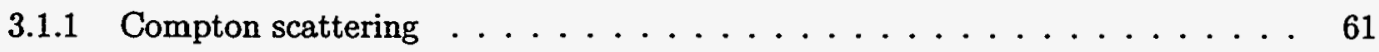

3.1 .2 Experimental asymmetry $\ldots \ldots \ldots \ldots \ldots \ldots \ldots$

3.2 Monte Carlo Simulation . . . . . . . . . . . . . . . . . . 66

3.2.1 The EGS4 Monte Carlo program . . . . . . . . . . . . . . . 66

3.2 .2 Čerenkov detector modeling . . . . . . . . . . . . . . 66

3.2 .3 Čerenkov response functions . . . . . . . . . . . . . . . 69

3.3 Polarization Determination $\ldots \ldots \ldots \ldots \ldots \ldots \ldots \ldots \ldots \ldots$

3.3 .1 Detector calibration $\ldots \ldots \ldots \ldots \ldots \ldots \ldots \ldots$

3.3.2 Čerenkov detector linearity . . . . . . . . . . . . . . . 84

3.3.3 Electronic noise and cross-talk . . . . . . . . . . . . . 88

3.3.4 Light polarization measurement . . . . . . . . . . . . . . . . . . 90

3.3.5 Compton asymmetry spectrum $\ldots \ldots \ldots \ldots \ldots \ldots$ 
3.3.6 Luminosity-weighted polarization results . . . . . . . . . . . . . . . . 95

4 Event Selection and Background Estimation $\quad 99$

4.1 Event selection $\ldots \ldots \ldots \ldots \ldots \ldots \ldots \ldots \ldots \ldots \ldots$

4.1 .1 Trigger . . . . . . . . . . . . . . . . . . . 100

4.1 .2 Event topologies . . . . . . . . . . . . . . . . . . 100

4.1 .3 Stage 1 filter . . . . . . . . . . . . . . . . . . . 103

4.1 .4 Stage 2 filter . . . . . . . . . . . . . . . . . . . . 104

4.1 .5 Stage 3 filter $\ldots \ldots \ldots \ldots \ldots \ldots \ldots \ldots \ldots$

4.1 .6 Polarization-related cuts . . . . . . . . . . . . . . . . . 113

4.1 .7 Measured asymmetry . . . . . . . . . . . . . . . . . . . 113

4.2 Background estimation . . . . . . . . . . . . . . . . . 113

4.2.1 Background estimation procedure and results . . . . . . . . . . . . 114

4.2 .2 Background asymmetry . . . . . . . . . . . . . . 116

5 Systematic Error Evaluation and Results $\quad 118$

5.1 Secondary Systematic Errors $\ldots \ldots \ldots \ldots \ldots \ldots \ldots \ldots \ldots$

5.1 .1 Background fraction . . . . . . . . . . . . . . . . . 119

5.1 .2 Polarization asymmetry . . . . . . . . . . . . . . . . . . . 119

5.1 .3 Energy asymmetry . . . . . . . . . . . . . . . . . . . . 120

5.1.4 Acceptance-efficiency product asymmetry . . . . . . . . . . . . . 120

5.1 .5 Luminosity asymmetry . . . . . . . . . . . . . . . . . . . . . . 121

5.2 Further Systematic Error Checks . . . . . . . . . . . . . . . . . . 122

5.2 .1 SLD-Compton spin precession . . . . . . . . . . . . . . . . 122

5.2 .2 Helicity bit transmission integrity . . . . . . . . . . . . . . . 123

5.2.3 Data acquisition synchronization . . . . . . . . . . . . . . 123

5.2 .4 Random number generator bias . . . . . . . . . . . . . . . . 126

5.2 .5 Sign of asymmetry $\ldots \ldots \ldots \ldots \ldots \ldots \ldots \ldots \ldots$

5.2 .6 Total systematic uncertainty $\ldots \ldots \ldots \ldots \ldots \ldots \ldots$

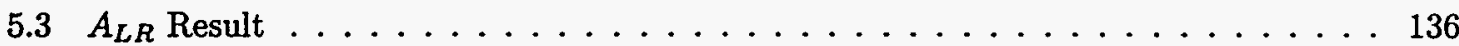

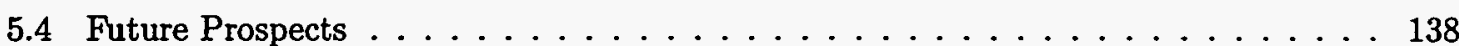

$\begin{array}{ll}\text { Bibliography } & 140\end{array}$ 


\section{List of Tables}

1.1 The known fermions grouped in left-handed weak isodoublets and right-handed weak isosinglets. . . . . . . . . . . . . . . . . . 4

1.2 Complete set of physical parameters specifying the tree-level Standard Model. . . . . 6

1.3 Fermion $Z$ vector and axial-vector couplings. . . . . . . . . . 8

3.1 Calculated analyzing powers for the Cerenkov detector. . . . . . . . . 79

3.2 A summary of the invasive measurements of $\mathcal{P}^{\gamma}$ made along the laser beam line. . . 91

3.3 Calculated analyzing powers and average measured raw asymmetries for each Cerenkov channel with $7.6 \mathrm{~mm}$ lead. . . . . . . . . . . . . . . . . . . 94

3.4 Contributions to systematic error in measurement of luminosity-weighted average beam polarization. . . . . . . . . . . . . . . . . 98

4.1 Results of the two independent hand scans. . . . . . . . . . . . . . . . 114

4.2 Results of background estimation techniques and expected numbers of misidentified

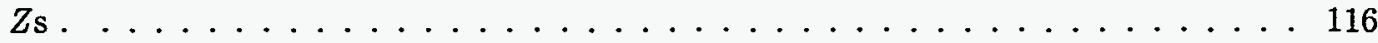

4.3 Left-right asymmetry in identified background events. . . . . . . . . . . . 117

5.1 Relationship between Compton laser bit pattern in data acquisition system and photon helicity at Compton IP. . . . . . . . . . . . . . . . . 128

5.2 Pockels cell sign for Compton Pockels cells used in 1992 run. . . . . . . . . . . 133

5.3 Evolution of electron helicity beam-code assignment throughout 1992 SLD run. . . . 134

5.4 Contributions to the systematic error in the measurement of $A_{L R} \ldots \ldots \ldots$ 


\section{List of Figures}

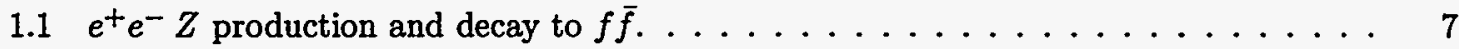

1.2 Total cross section for $e^{+} e^{-} \rightarrow \mu^{+} \mu^{-}$as a function of center-of-mass energy. . . . . 8

1.3 Leading-order QED initial-state radiative corrections to the $Z$ line shape. . . . . . 11

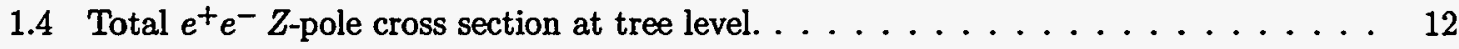

1.5 First-order virtual QED corrections to the process $e^{+} e^{-} \rightarrow f \bar{f}$ at the $Z$ pole. . . . 15

1.6 First-order virtual electroweak corrections to $e^{+} e^{-} \rightarrow f \bar{f}$. . . . . . . . 15

1.7 Dependence of $\sin ^{2} \theta_{W}^{\text {eff }}\left(M_{Z}^{2}\right)$ on top and Higgs masses. . . . . . . . . . 18

1.8 Dependence of $\left.\sin ^{2} \theta_{W}\right|_{S}$ on top and Higgs masses. .............. 18

$1.9 A_{L R}$ as a function of center-of-mass energy with and without initial-state radiation. 22

1.10 The fully corrected $A_{L R}$ as a function of top and Higgs mass. . . . . . . . . 23

1.11 Error in $A_{L R}$ measurement as a function of $Z$ sample, polarization, and polarization measurement error. . . . . . . . . . . . . . . . . . . 25

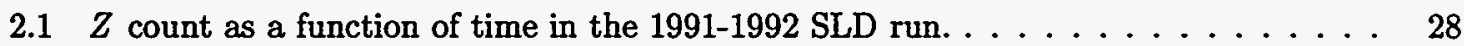

2.2 The polarized SLC. . . . . . . . . . . . . . . . . . . . 29

2.3 The SLC polarized source. . . . . . . . . . . . . . . . . 30

2.4 The band structure and energy-level diagram for GaAs. . . . . . . . . . . . 31

2.5 The SLC polarized electron gun. . . . . . . . . . . . . . . . . 32

2.6 Polarization vs. wavelength for various GaAs photocathodes. . . . . . . . . . . 32

2.7 The north damping ring and spin-rotation solenoids. . . . . . . . . . . . . . 34

2.8 Longitudinal polarization at the IP as a function of north arc vertical launch angle

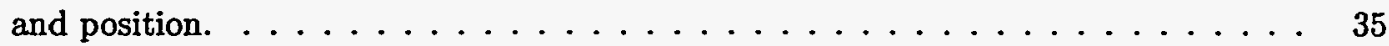

2.9 The linac Møller polarimeter. . . . . . . . . . . . . . . . . 36

2.10 Schematic of the extraction-line energy spectrometer. . . . . . . . . . . . 37 
2.11 Schematic diagram of the Compton polarimeter. . . . . . . . . . . . . 39

2.12 The Compton source laser bench layout. . . . . . . . . . . . . . . 40

2.13 The Compton $\mathbb{P}$ region. . . . . . . . . . . . . . . . . . 42

2.14 Overhead view of the Compton Čerenkov and proportional tube detectors. . . . . . 43

2.15 Schematic of Čerenkov detector phototube base. . . . . . . . . . . . 45

2.16 The normalized linearity of a typical phototube/base combination for $\epsilon_{2}=600 \mathrm{~V}$ and three different $\epsilon_{1}$ values. . . . . . . . . . . . . . . . . . 47

2.17 Linearity of same phototube/base with $\epsilon_{2}=1000 \mathrm{~V}$ for different $\epsilon_{1}$ values. . . . . . 47

2.18 The Compton proportional tube detector. . . . . . . . . . . . . . 48

2.19 Quadrant schematic view of the SLD detector. . . . . . . . . . . . 52

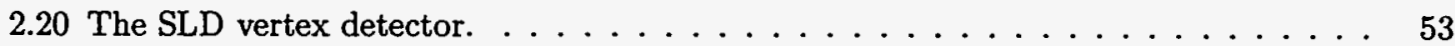

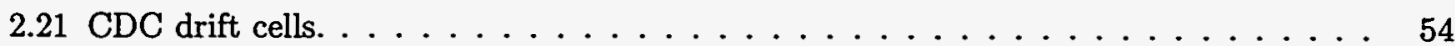

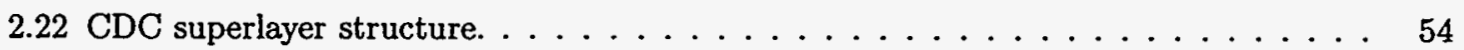

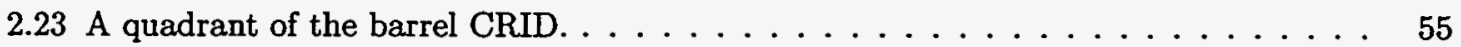

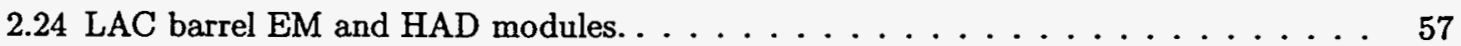

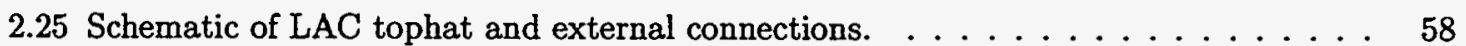

2.26 Section detail of the WIC $\ldots \ldots \ldots \ldots \ldots \ldots \ldots \ldots \ldots$

2.27 The LMSAT and MASC small angle calorimeters. . . . . . . . . . . 60

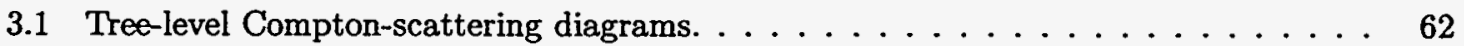

3.2 The unpolarized cross section and longitudinal and transverse asymmetries for Compton scattering of a $2.33 \mathrm{eV}$ photon with a $46 \mathrm{GeV}$ electron. . . . . . . . . . 64

3.3 Construction drawing of the front section of the Cerenkov detector. . . . . . . . 67

3.4 Passage of a $28 \mathrm{GeV}$ electron through Čerenkov channel 1 as viewed from above. . . 70

3.5 The same interaction viewed from the side. . . . . . . . . . . . 70

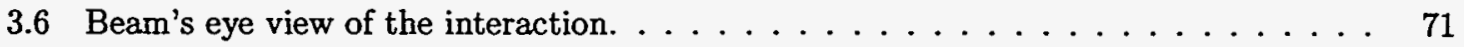

3.7 EGS-derived response function for Čerenkov channel 6 with no $\mathrm{Pb}$ shield. . . . . . 72

3.8 Response function for channel 6 with $7.6 \mathrm{~mm} \mathrm{~Pb}$ shielding. . . . . . . . . . 72

3.9 The Compton asymmetry and unpolarized cross section plotted vs. transverse distance from the undeflected beamline. . . . . . . . . . . . . . . . 73

3.10 Cubic spline fit to channel 6 edge scan data with lead shielding in front of detector. . 76

3.11 Fit of Monte Carlo to spline fit of data. . . . . . . . . . . . . . . . 76

3.12 Cubic spline fit to channel 6 edge scan data with no lead shielding in front of detector. 77 


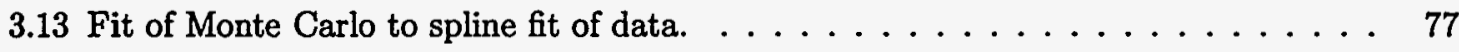

3.14 The $+5 \mathrm{mr}$ simulated $8.5 \mathrm{~mm}$ lead channel 6 edge scan (circles) is placed on the same horizontal scale with the best fit of aligned simulated edge scan to data fit. . . . . 82

$3.15 \mathrm{The}+5 \mathrm{mr}$ edge scan (circles) shifted to provide the best fit with data fit. . . . . 82

3.16 Plot of channel 6 analyzing power as a function of channel misalignment angle. . . . 83

3.17 Channel 7 analyzing power $v s$. misalignment angle. . . . . . . . . . 83

3.18 Time history of the ratio of background-subtracted pulse heights in channels 7 and 2 plotted vs. polarimeter run number. . . . . . . . . . . . . . 84

$3.19 A_{0}$ plotted as a function of polarimeter run number. $\ldots \ldots \ldots \ldots$

3.20 The ratio of polarization measured in PTD to that in Cerenkov detector plotted $v s$. PTD signal-plus-background pulse height averaged over seven PTD channels. . . . . 86

3.21 Measured Compton asymmetry in Čerenkov channel 6 relative to corrected measured PTD polarization, plotted $v$ s. channel 6 pulse height. . . . . . . . . . .

3.22 Measured Compton asymmetry in Cerenkov channel 7 relative to corrected measured PTD polarization, plotted $v s$. channel 7 pulse height. . . . . . . . . . 87

3.23 Relative asymmetry measured in Čerenkov channel 6 in the nominal pulse-height range. 89

3.24 Relative asymmetry measured in Čerenkov channel 7 in the nominal pulse-height range. 89

3.25 Schematic of experimental setup for measuring light circular polarization and handedness. . . . . . . . . . . . . . . . . . . 90

3.26 Schematic of the laser optical transport line. . . . . . . . . . . . . 92

3.27 Circular light polarization measured on the laser bench throughout the 1992 run. . . 93

3.28 Comparison of observed Čerenkov channel asymmetries, averaged over all polarizedbeam running, with the theoretical Compton asymmetry function. . . . . . . 95

3.29 (a) Time history of electron polarization associated in time with each $Z$ event. (b) Distribution of electron polarization associated in time with each $Z$ event. . . . . .

4.1 (a) Distribution of $N_{L A C}$ for all triggered events of four sample runs. (b) Distribution of $E_{L A C}$ for the runs. (c) $E_{T O T} v s . E_{W I C}^{e n d}$ after cuts 1 and $2 \ldots \ldots \ldots \ldots$

4.2 (a) Distribution of $E_{I M B}$ after cuts 1, 2, and 3. (b) $E_{I M B}$ vs. $S P H E$ after cuts 1, 2,

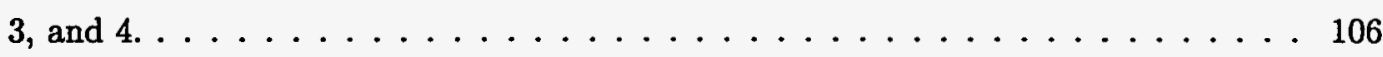

4.3 (a) Distribution of $N_{L A C}$ for events passing stage 1 filter. (b) Distribution of $E_{L A C}$ for these events. (c) $E_{T O T} v s$. $E_{W I C}^{e n d}$ for the same events. (d) $E_{I M B} v s$. SPHE for the events. . . . . . . . . . . . . . . . . . . . . . . . 107 


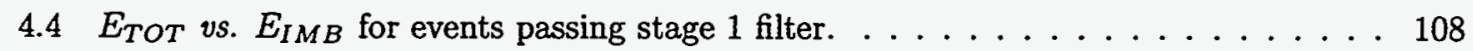

$4.5 S_{4}$ vs. $\Theta_{\max }$ for all events passing stage 1 filter. . . . . . . . . . . . 109

4.6 (a) Distribution of $E_{T O T}$ for events identified by second-stage filter as wide-angle Bhabhas in the region $\Theta_{\text {max }} \leq 44$. (b) Distribution for those wide-angle Bhabhas

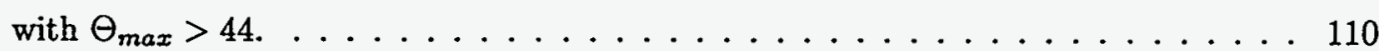

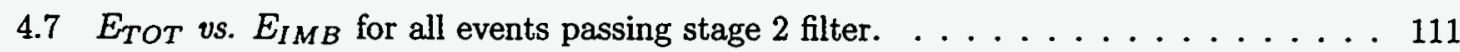

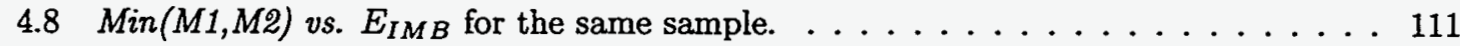

4.9 Top: Distribution of $E_{T O T}$ in the final event sample. Center: Distribution of $E_{I M B}$.

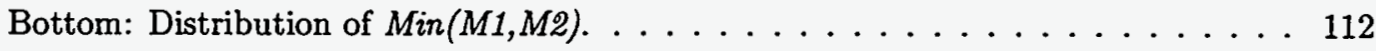

4.10 Likelihood function for number of background events. . . . . . . . . . . 115

5.1 Experimental setup for test of Pockels cell sign. . . . . . . . . . . . . . . . 129

5.2 Orientation required of quarter-wave plate to convert horizontal linear light (into page) to left-handed light. . . . . . . . . . . . . . . . . . . 129

5.3 Experimental setup for Fresnel-method slow-axis determination. . . . . . . . . . 131

5.4 Left: Linear polarization orientation before total internal reflection. Right: Quarterwave-plate orientation for extinction. . . . . . . . . . . . . . . 131

5.5 Experimental setup for CD-method slow-axis determination. . . . . . . . . . . . 132

5.6 Čerenkov channel 7 raw signal for 01 electron beam code and 01 and 10 laser beam code. . . . . . . . . . . . . . . . . . . . . . . . . . . 135

5.71992 SLD $A_{L R}$ measurement compared with recent average $\tau$-polarization measurements from the four LEP experiments. . . . . . . . . . . . . . . . . 138

5.8 Precision in the measurement of $\sin ^{2} \theta_{W}$ as a function of $Z$ count, polarization, and polarization-measurement precision. . . . . . . . . . . . . . . . . 139 


\section{Chapter 1}

\section{The Left-Right Asymmetry}

The left-right asymmetry $A_{L R}$ is a direct and sensitive probe of the Standard Model of electroweak interactions. The measurement of $A_{L R}$ is the principal goal of the ongoing SLC/SLD program, and provides the world's most precise experimental determination of the effective weak mixing angle at the $Z$ pole [1].

\subsection{The Electroweak Standard Model}

Since its inception in the 1960s, the Standard Model of electroweak interactions [2] has proved to be a numbingly successful theory. All experimental tests of the model have become verification of its correctness, and have determined its several unpredicted parameters with ever-increasing precision.

\subsubsection{The electroweak interaction}

The Standard Model uses an "isospin" triplet of gauge fields, constructed with the Pauli spin matrices, in conjunction with a "hypercharge" singlet field, analogous to the electromagnetic interaction, to create charged and neutral weak currents [3]. The weak hypercharge $Y$ and third component $T^{3}$ of weak isospin are related to electric charge by the expression

$$
Q=T^{3}+\frac{Y}{2},
$$

yielding the current relation

$$
j_{\mu}^{e m}=J_{\mu}^{3}+\frac{1}{2} j_{\mu}^{Y}
$$


among the electromagnetic current $j^{e m}$, the neutral weak isospin current $J_{\mu}^{3}=\bar{\psi}_{f} \gamma_{\mu} \frac{1}{2}\left(1-\gamma^{5}\right) T^{3} \psi_{f}$, and the weak hypercharge current $j_{\mu}^{Y}=\bar{\psi}_{f} \gamma_{\mu} Y \psi_{f}$. The basic electroweak interaction is then taken to be

$$
-i g\left(J^{i}\right)^{\mu} W_{\mu}^{i}-i \frac{g^{\prime}}{2}\left(j^{Y}\right)^{\mu} B_{\mu}
$$

where $W_{\mu}^{i}$ is a vector isotriplet coupled to the left-handed $S U(2)_{L}$ weak isospin current $J_{\mu}^{i}$ with strength $g$, and $B_{\mu}$ is a vector singlet coupled to the $U(1)$ weak hypercharge current $j_{\mu}^{Y}$ with coupling constant $g^{\prime}$. The charged weak vector bosons $\mathrm{W}^{ \pm}$, which couple only to left-handed fermions, are described by a composition of the isotriplet fields $W_{\mu}^{1}$ and $W_{\mu}^{2}$ :

$$
W_{\mu}^{ \pm}=\sqrt{\frac{1}{2}}\left(W_{\mu}^{1} \mp W_{\mu}^{2}\right)
$$

We describe the mass eigenstates $A_{\mu}$ and $Z_{\mu}$ of the photon and the neutral weak vector boson, the $Z$, as mixtures of the neutral fields $W_{\mu}^{3}$ and $B_{\mu}$ which we will find to be rotations in the $\left(W_{\mu}^{3}, B_{\mu}\right)$ plane by the angle $\theta_{W}$ :

$$
\begin{aligned}
& A_{\mu}=B_{\mu} \cos \theta_{W}+W_{\mu}^{3} \sin \theta_{W} \\
& Z_{\mu}=-B_{\mu} \sin \theta_{W}+W_{\mu}^{3} \cos \theta_{W} .
\end{aligned}
$$

The value of the weak mixing angle $\theta_{W}$ is not predicted by the Standard Model and must be determined experimentally. The photon is observed to couple to right-handed as well as left-handed fermions; therefore, since $Z_{\mu}$ is composed of the same fields as $A_{\mu}$, the $Z$ must also couple to both handednesses. Using the parametrization of Eq. 1.3, we may write the electroweak neutral-current interaction as

$$
\begin{aligned}
-i g J_{\mu}^{3}\left(W^{3}\right)^{\mu}-i \frac{g^{\prime}}{2} j_{\mu}^{Y} B^{\mu} & = \\
& -i\left(g \sin \theta_{W} J_{\mu}^{3}+g^{\prime} \cos \theta_{W} \frac{j_{\mu}^{Y}}{2}\right) A^{\mu} \\
& -i\left(g \cos \theta_{W} J_{\mu}^{3}-g^{\prime} \sin \theta_{W} \frac{j_{\mu}^{Y}}{2}\right) Z^{\mu}
\end{aligned}
$$

Identifying the first line above as the electromagnetic interaction and comparing with Eq. 1.1 yields the relation

$$
g \sin \theta_{W}=g^{\prime} \cos \theta_{W}=e .
$$


Using Eqs. 1.1 and 1.5, we may express the weak neutral-current interaction of Eq. 1.4 as

$$
-i \frac{g}{\cos \theta_{W}} J_{\mu}^{N C} Z^{\mu}
$$

in terms of a weak neutral current $J_{\mu}^{N C}$ given by

$$
J_{\mu}^{N C} \equiv J_{\mu}^{3}-\sin ^{2} \theta_{W} j_{\mu}^{e m}
$$

Eqs. 1.1 and 1.7 complete the specification of the weak neutral current in terms of the neutral weak isospin and weak hypercharge currents.

Low-energy charged-current phenomena are usually described using the empirical invariant amplitude

$$
\mathcal{M}^{C C}=\frac{4 G_{F}}{\sqrt{2}} J^{\mu} J_{\mu}^{\dagger}
$$

where $J_{\mu} \equiv \frac{1}{2}\left(J_{\mu}^{1}+i J_{\mu}^{2}\right)$ and $G_{F}=1.16637(2) \times 10^{-5}(\mathrm{GeV})^{-2}$ is the Fermi constant, one of the best-measured physical constants of nature. Rewriting the charged-current interaction of Eq. 1.2 as

$$
-i \frac{g}{\sqrt{2}}\left(J^{\mu} W_{\mu}^{+}+J^{\mu \dagger} W_{\mu}^{-}\right)
$$

leads to the amplitude

$$
\mathcal{M}^{C C}=\left(\frac{g}{\sqrt{2}} J_{\mu}\right)\left(\frac{1}{M_{W}^{2}}\right)\left(\frac{g}{\sqrt{2}} J^{\mu \dagger}\right)
$$

for the low- $q^{2} W$-mediated charged-current interaction. Comparison of Eq. 1.9 with Eq. 1.8 yields the tree-level relationship

$$
\frac{G_{F}}{\sqrt{2}}=\frac{g^{2}}{8 M_{W}^{2}}
$$

The known quarks and leptons are shown in Table 1.1. The left-handed fermions are arranged in weak isodoublets with $T^{3}=+\frac{1}{2}$ and $T^{3}=-\frac{1}{2}$ for the top and bottom entries respectively. The righthanded fermions are arranged in weak isosinglets with $T^{3}=0$. The top quark, as yet undiscovered, is shown with its expected quantum numbers.

\subsubsection{Higgs mechanism and boson masses}

Thus far, we have demonstrated the possibility of expressing the observed charged and neutral weak currents in terms of $S U(2)_{L}$ and $U(1)_{Y}$ currents, with no mention of particle masses or explanation of the rotation of Eq. 1.3. Of course, the success of the theory depends on the correct prediction or 


$$
\begin{aligned}
& \left(\begin{array}{c}
\nu_{e} \\
e
\end{array}\right)_{L}\left(\begin{array}{l}
u \\
d
\end{array}\right)_{L}(e)_{R} \quad(u)_{R} \quad(d)_{R} \\
& \left(\begin{array}{c}
\nu_{\mu} \\
\mu
\end{array}\right)_{L}\left(\begin{array}{l}
c \\
s
\end{array}\right)_{L}(\mu)_{R} \quad(c)_{R} \quad(s)_{R} \\
& \left(\begin{array}{c}
\nu_{\tau} \\
\tau
\end{array}\right)_{L}\left(\begin{array}{l}
t \\
b
\end{array}\right)_{L}(\tau)_{R} \quad(t)_{R} \quad(b)_{R}
\end{aligned}
$$

Table 1.1: The known fermions grouped in left-handed weak isodoublets and right-handed weak isosinglets.

accommodation of the observed masses. Requirement of local gauge invariance and renormalizability forbids the introduction of mass terms such as $M^{2} W_{\mu} W^{\mu}$ into the electroweak Lagrangian. A way to properly generate particle masses is through the technique of spontaneous symmetry breaking. The Higgs mechanism is the proposal to introduce four scalar fields $\phi_{i}$ and add to the Lagrangian an $S U(2)_{L} \times U(1)_{Y}$ gauge-invariant term

$$
\mathcal{L}=\left|\left(i \partial_{\mu}-g \vec{T} \cdot \vec{W}_{\mu}-g^{\prime} \frac{Y}{2} B_{\mu}\right) \phi\right|^{2}-V(\phi),
$$

where the $\vec{T}$ matrices are the weak isospin generators. Gauge invariance of this expanded Lagrangian requires that the $\phi_{i}$ inhabit $S U(2)_{L} \times U(1)_{Y}$ multiplets, most conveniently chosen to be the $Y=1$ isodoublet

$$
\phi=\left(\begin{array}{l}
\left(\phi_{1}+i \phi_{2}\right) / \sqrt{2} \\
\left(\phi_{3}+i \phi_{4}\right) / \sqrt{2}
\end{array}\right)
$$

We choose the Higgs potential

$$
V(\phi)=\mu^{2} \phi^{\dagger} \phi+\lambda\left(\phi^{\dagger} \phi\right)^{2}
$$

with $\mu^{2}<0$ and $\lambda>0$, creating an $S U(2)$-invariant locus of $V(\phi)$ minima for values of $\phi$ such that

$$
\phi^{\dagger} \phi=\frac{1}{2}\left(\phi_{1}^{2}+\phi_{2}^{2}+\phi_{3}^{2}+\phi_{4}^{2}\right)=-\frac{\mu^{2}}{2 \lambda} \equiv \frac{1}{2} v^{2} .
$$

It is easily shown that four independent fields $\theta_{1}, \theta_{2}, \theta_{3}$, and $h$ can be used to parametrize fluctuations from the vacuum $\phi_{0}$,

$$
\phi_{0} \equiv \sqrt{\frac{1}{2}}\left(\begin{array}{l}
0 \\
v
\end{array}\right)
$$


with the form

$$
\phi(x)=e^{i 2 \vec{T} \cdot \vec{\theta}(x) / v}\left(\begin{array}{c}
0 \\
\frac{v+h(x)}{\sqrt{2}}
\end{array}\right) ;
$$

then we are free to gauge away the three fields $\theta_{i}$, leaving

$$
\phi(x)=\sqrt{\frac{1}{2}}\left(\begin{array}{c}
0 \\
v+h(x)
\end{array}\right)
$$

By substituting this form of the scalar field into the Lagrangian of Eq. 1.11, we find the terms $\frac{1}{2}\left(\partial_{\mu} h\right)^{2}$ and $-\lambda v^{2} h^{2}$, corresponding to kinetic energy and mass of the scalar particle $h$, the Higgs particle, as well as Higgs interactions with the gauge bosons. The masses of the gauge bosons are obtained upon substitution of the vacuum expectation value from Eq. 1.12 into the Lagrangian:

$$
\left(\frac{1}{2} v g\right)^{2} W_{\mu}^{+} W^{-\mu}+\frac{1}{8} v^{2}\left[g W_{\mu}^{3}-g^{\prime} B_{\mu}\right]^{2}+0\left[g^{\prime} W_{\mu}^{3}+g B_{\mu}\right]^{2}
$$

where the first term is to be compared with the expected $M_{W}^{2} W^{+} W^{-}$for a charged boson, giving

$$
M_{W}=\frac{1}{2} v g,
$$

and the last two terms are chosen to be orthogonal in the $\left(W_{\mu}^{3}, B_{\mu}\right)$ basis and are identified with the $Z_{\mu}$ and $A_{\mu}$ mass terms $\frac{1}{2} M_{Z}^{2} Z_{\mu}^{2}$ and $\frac{1}{2} M_{A}^{2} A_{\mu}^{2}$, yielding, upon normalization,

$$
\begin{aligned}
& A_{\mu}=\frac{g^{\prime} W_{\mu}^{3}+g B_{\mu}}{\sqrt{g^{2}+g^{\prime 2}}} \text { with } M_{A}=0 \\
& Z_{\mu}=\frac{g W_{\mu}^{3}-g^{\prime} B_{\mu}}{\sqrt{g^{2}+g^{\prime 2}}} \text { with } M_{Z}=\frac{1}{2} v \sqrt{g^{2}+g^{2}} .
\end{aligned}
$$

Using the relationships of Eq. 1.5 to express the couplings $g$ and $g^{\prime}$ in terms of the weak mixing angle, we validate the ad hoc rotation of Eq. 1.3. In addition, using the masses of Eqs. 1.14 and 1.15 , we obtain the result

$$
\frac{M_{W}}{M_{Z}}=\cos \theta_{W}
$$

Thus, the Higgs mechanism not only provides a mixing of the weak isospin and hypercharge fields to produce the observable gauge bosons, but also makes a testable prediction of the ratio of the masses of the charged and neutral gauge bosons. Unfortunately, the masses themselves, as well as the fermion and Higgs masses, are not predicted by the theory and must be determined experimentally. 


\subsubsection{Electroweak parameters}

As we have seen in the preceding sections, all tree-level electroweak phenomena can be described using three parameters: the $S U(2)_{L}$ and $U(1)$ coupling constants $g$ and $g^{\prime}$ and the Higgs field vacuum expectation value $\left\langle\phi_{0}\right\rangle$. In order to precisely test the Standard Model, we must accurately determine these parameters and use them to make a testable prediction. Since $g, g^{\prime}$, and $\left\langle\phi_{0}\right\rangle$ are not directly measurable, we use the tree-level Standard Model relations to define a complete physical set of parameters. As it is advantageous to use observables with the smallest associated measurement uncertainties, we choose the standard set of physical electroweak parameters as follows: from Eq. 1.5, we have

$$
\alpha=\frac{g^{2} g^{\prime 2}}{4 \pi\left(g^{2}+g^{\prime 2}\right)},
$$

where $\alpha=e^{2} / 4 \pi$ is the electromagnetic fine-structure constant; from Eqs. 1.10, 1.12, and 1.14,

$$
G_{F}=\frac{1}{2 \sqrt{2}\left\langle\phi_{0}\right\rangle^{2}}
$$

and from Eqs. 1.12 and 1.15 we have

$$
M_{Z}=\frac{1}{\sqrt{2}}\left\langle\phi_{0}\right\rangle \sqrt{g^{2}+g^{\prime 2}}
$$

The measured values of these observables [4] are shown in Table 1.2. We will return later in the chapter to the use of $\sin ^{2} \theta_{W}$ as a Standard Model test.

\begin{tabular}{|c|c|c|}
\hline Parameter & Measured Value & Precision \\
\hline$\alpha$ & $1 / 137.0359895(61)$ & $4.5 \times 10^{-8}$ \\
$G_{F}$ & $1.16637(2) \times 10^{-5}(\mathrm{GeV})^{-2}$ & $1.7 \times 10^{-5}$ \\
$M_{Z}$ & $91.187(7) \mathrm{GeV}$ & $7.7 \times 10^{-5}$ \\
\hline
\end{tabular}

Table 1.2: Complete set of physical parameters specifying the tree-level Standard Model.

In addition to these tree-level parameters, the fermion masses, as well as the Higgs mass and couplings, are not specified in the Standard Model. These quantities appear in radiative corrections to the zero-order processes and hence affect the value of precision electroweak measurements. Thus, we can gain insight into some of these still-unknown parameters, such as the top-quark and Higgs masses, through comparison of experimental results with higher-order theoretical predictions. 


\subsection{Z Production and Decay}

With the advent of SLC and LEP, the electroweak interaction is now directly accessible through resonant production of the $Z$ boson. The $Z$ is a rich source of physics, producing all known fermions in its decay, and provides information on the structure of the Standard Model through its couplings to the initial- and final-state particles. The process $e^{+} e^{-} \rightarrow Z \rightarrow f \bar{f}$ is pictured in Fig. 1.1.

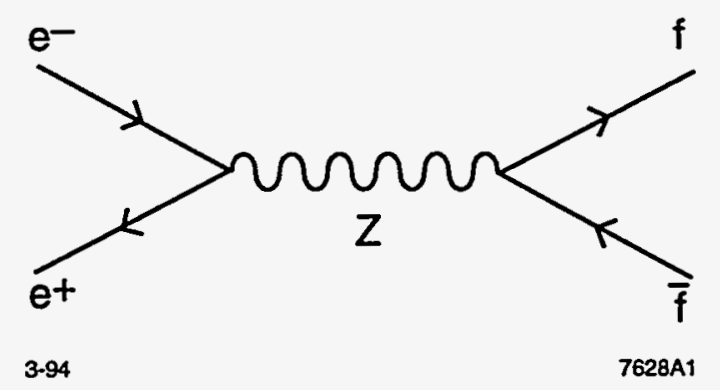

Figure 1.1: $e^{+} e^{-} Z$ production and decay to $f \bar{f}$.

\subsubsection{The electroweak neutral current}

From Eqs. 1.6 and 1.7 we have the $Z \rightarrow f \bar{f}$ neutral-current interaction

$$
-i \frac{g}{\cos \theta_{W}}\left(J_{\mu}^{3}-\sin ^{2} \theta_{W} j_{\mu}^{e m}\right) Z^{\mu}
$$

or, in terms of the electroweak quantum numbers,

$$
-i \frac{g}{\cos \theta_{W}} \bar{\psi}_{f} \gamma^{\mu}\left[\frac{1}{2}\left(1-\gamma^{5}\right) T_{f}^{3}-\sin ^{2} \theta_{W} Q_{f}\right] \psi_{f} Z_{\mu}
$$

The vertex factor is conventionally expressed in terms of vector and axial-vector couplings to the $Z$ :

$$
-i \frac{g}{\cos \theta_{W}} \gamma^{\mu} \frac{1}{2}\left(c_{V}^{f}-c_{A}^{f} \gamma^{5}\right)
$$

where

$$
\begin{aligned}
c_{V}^{f} & =T_{f}^{3}-2 \sin ^{2} \theta_{W} Q_{f} \\
c_{A}^{f} & =T_{f}^{3}
\end{aligned}
$$


with $Q_{f}$ and $T_{f}^{3}$ the charge and third component of weak isospin of the fermion. The vector and axial-vector couplings of the fermions are listed in Table 1.3. As is evident from the $c_{V}^{f}-c_{A}^{f} \gamma^{5}$ form of 1.18 , nonzero values of $c_{V}^{e}$ and $c_{A}^{e}$ guarantee parity violation in electronic $Z$ production.

\begin{tabular}{|c|r|c|}
\hline$f$ & $c_{A}^{f}$ & \multicolumn{1}{|c|}{$c_{V}^{f}$} \\
\hline$\nu_{e}, \nu_{\mu}, \nu_{\tau}$ & $\frac{1}{2}$ & $\frac{1}{2}$ \\
$e^{-}, \mu, \tau^{-}$ & $-\frac{1}{2}$ & $-\frac{1}{2}+2 \sin ^{2} \theta_{W}$ \\
$\mathrm{u}, \mathrm{c}, \mathrm{t}$ & $\frac{1}{2}$ & $\frac{1}{2}-\frac{4}{3} \sin ^{2} \theta_{W}$ \\
$\mathrm{~d}, \mathrm{~s}, \mathrm{~b}$ & $-\frac{1}{2}$ & $-\frac{1}{2}+\frac{2}{3} \sin ^{2} \theta_{W}$ \\
\hline
\end{tabular}

Table 1.3: Fermion- $Z$ vector and axial-vector couplings.

\subsection{2 $Z$ cross section}

The process $e^{+} e^{-} \rightarrow Z \rightarrow f \bar{f}$ gives rise to a large resonance in the $e^{+} e^{-}$cross section at the $Z$ mass. The energy dependence of the $e^{+} e^{-}$cross section to $\mu$ pairs is shown in Fig. 1.2. As is clear from

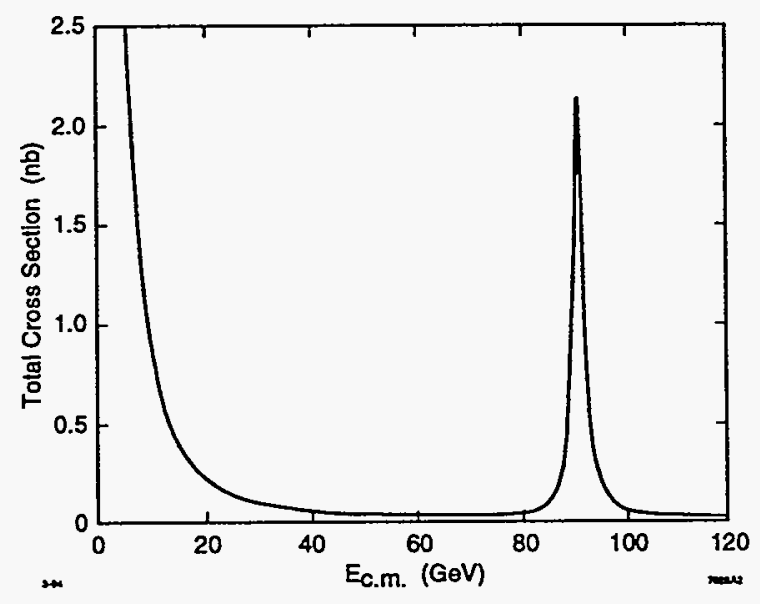

Figure 1.2: Total cross section for $e^{+} e^{-} \rightarrow \mu^{+} \mu^{-}$as a function of center-of-mass energy.

the figure, the $Z$ peak dominates the photon-mediated tail of the cross section. This factor of $\sim 800$ in cross section allows the neglect of the QED processes in the calculation of the peak cross section. In addition, at the $Z$ pole, the $\gamma-Z$ interference terms vanish or become negligible, leaving only the pure $Z$-exchange terms. Our purposes require a polarization-dependent cross section, where we define the beam polarization $\mathcal{P}$ in a direction $\hat{n}$ as the difference between the number of electron (or 
positron) spins parallel and antiparallel to $\hat{n}$ divided by the total number of particles in the beam:

$$
\mathcal{P}(\hat{n})=\frac{N_{e}(\text { spins parallel to } \hat{n})-N_{e}(\text { spins antiparallel to } \hat{n})}{N_{e}(\text { spins parallel to } \hat{n})+N_{e}(\text { spins antiparallel to } \hat{n})}
$$

The beam polarization $\mathcal{P}$ is taken to be $\mathcal{P}(\hat{s})$ where $\hat{s}$ is the direction for which $\mathcal{P}(\hat{n})$ is maximized.

With these simplifications and definitions, we may now write the polarization-dependent treelevel $Z$-pole differential cross section for $e^{+} e^{-} \rightarrow f \bar{f}$ :

$$
\begin{aligned}
\frac{d \sigma}{d \Omega}= & \frac{\alpha^{2}}{4 \sin ^{4} 2 \theta_{W}} \frac{s}{\left(s-M_{Z}^{2}\right)^{2}+\Gamma_{Z}^{2} s^{2} / M_{Z}^{2}} \times \\
& \left\{\left(1-\mathcal{P}_{z}^{+} \mathcal{P}_{z}^{-}\right)\left[\left(1+c^{2}\right)\left(c_{V}^{e}+c_{A}^{e^{2}}\right)\left(c_{V}^{f^{2}}+c_{A}^{f^{2}}\right)-8 c c_{V}^{e} c_{A}^{e} c_{V}^{f} c_{A}^{f}\right]\right. \\
& +\left(\mathcal{P}_{z}^{+}-\mathcal{P}_{z}^{-}\right)\left[2\left(1+c^{2}\right) c_{V}^{e} c_{A}^{e}\left(c_{V}^{f^{2}}+c_{A}^{f^{2}}\right)+4 c\left(c_{V}^{e}{ }^{2}+c_{A}^{e}\right) c_{V}^{f} c_{A}^{f}\right] \\
& \left.+\mathcal{P}_{t}^{+} \mathcal{P}_{t}^{-} \cos \Phi\left(1-c^{2}\right)\left(c_{V}^{e^{2}}-{c_{A}^{e}}^{2}\right)\left(c_{V}^{f^{2}}+c_{A}^{f^{2}}\right)\right\}
\end{aligned}
$$

where $\alpha=e^{2} / 4 \pi$ is the electromagnetic fine-structure constant; $s$ is the square of the center-of-mass collision energy; $\Gamma_{Z}$ is the $Z$ resonance width; $c$ is the cosine of the polar angle of the outgoing fermion; $\mathcal{P}_{z}^{+}$and $\mathcal{P}_{z}^{-}$are the signed longitudinal positron and electron beam polarizations, with $\mathcal{P}_{z}= \pm 1$ corresponding to right- and left-handed particles respectively; $\mathcal{P}_{t}^{+}$and $\mathcal{P}_{t}^{-}$are the transverse positron and electron polarizations; and $\Phi$ is defined by $\Phi=2 \phi-\phi^{-}-\phi^{+}$, with $\phi$ the azimuthal angle of the outgoing fermion and $\phi^{ \pm}$the azimuth of positron and electron transverse polarization directions. The cross section simplifies somewhat in the case of negligible transverse and positron polarization:

$$
\begin{aligned}
\frac{d \sigma}{d \Omega} & =k\left\{\left[\left(1+c^{2}\right)\left(c_{V}^{e}{ }^{2}+c_{A}^{e}{ }^{2}\right)\left(c_{V}^{f^{2}}+c_{A}^{f^{2}}\right)-8 c c_{V}^{e} c_{A}^{e} c_{V}^{f} c_{A}^{f}\right]\right. \\
-\mathcal{P}_{z}^{-} & {\left.\left[2\left(1+c^{2}\right) c_{V}^{e} c_{A}^{e}\left(c_{V}^{f^{2}}+c_{A}^{f^{2}}\right)+4 c\left(c_{V}^{e}+c_{A}^{e}\right) c_{V}^{f} c_{A}^{f}\right]\right\}, }
\end{aligned}
$$

with

$$
k \equiv \frac{\alpha^{2}}{4 \sin ^{4} 2 \theta_{W}} \frac{s}{\left(s-M_{Z}^{2}\right)^{2}+\Gamma_{Z}^{2} s^{2} / M_{Z}^{2}}
$$


The effects of photon- $Z$ interference are incorporated by adding to this result the interference crosssection terms

$$
\left.\frac{d \sigma}{d \Omega}\right|_{\gamma Z}=-2 Q_{f}\left(1-\frac{M_{Z}^{2}}{s}\right) k\left\{\left[\left(1+c^{2}\right) c_{V}^{e} c_{V}^{f}+2 c c_{A}^{e} c_{A}^{f}\right]-\mathcal{P}_{z}^{-}\left[\left(1+c^{2}\right) c_{A}^{e} c_{V}^{f}+2 c c_{V}^{e} c_{A}^{f}\right]\right\}
$$

where $Q_{f}$ is the electric charge of the fermion. We see, as noted earlier, that the interference cross section vanishes at the $Z$ pole; however, the difference in energy dependence between the interference cross section and the $Z$-exchange cross section of Eq. 1.20 implies an energy dependence in the left-right asymmetry, to be discussed later in the chapter.

The cross section of Eq. 1.20 has a polarization-dependent part; this fact, coupled with the requirement of opposite-helicity $e^{+} e^{-}$to create the spin-1 $Z$, confirms our prediction of parity violation in electronic $Z$ production. In addition, both the unpolarized and polarization-dependent parts contain polar-angle-symmetric and -antisymmetric terms, implying that even unpolarized $Z$ decays will manifest a difference in fermionic cross section between the forward and backward hemispheres.

\subsection{Radiative Corrections to $Z$ Production}

The discussion thus far has included electroweak effects at only tree level. The cross section created and measured in the laboratory differs from the zero-order process by initial-state radiation and by virtual corrections to the $e^{+} e^{-}-Z$ vertex and $Z$ propagator. These radiative corrections must be understood and incorporated to make a meaningful comparison of experimental data with theory [5].

\subsubsection{Initial-state radiation}

The effect of initial-state QED radiation on the $Z$ line shape is a topic of crucial importance to the experimental extraction of the resonance parameters and the understanding of other precision electroweak results. The tree-level initial-state QED processes that affect the $Z$ line shape are shown in Fig. 1.3. The general influence of these diagrams is to smear out and lower the energy of the initial $e^{+} e^{-}$, pushing the $Z$ peak to higher energy and changing the shape of the resonance. We let $1-z_{1}$ and $1-z_{2}$ represent the fraction of beam energy radiated by the electron and positron prior to collision. The corrected cross section can then be written in terms of $z_{1}$ - and $z_{2}$-dependent

distribution functions $D_{e}(z)$, indicating the probability of the electron or positron to have a fraction 


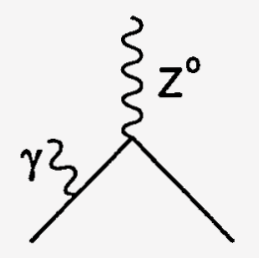

3-90

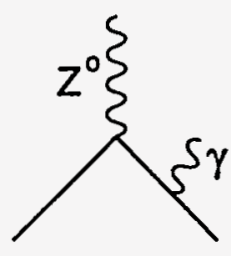

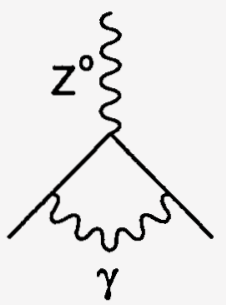

6581A10

Figure 1.3: Leading-order QED initial-state radiative corrections to the $Z$ line shape.

$z$ of its initial energy, and the uncorrected cross section $\sigma_{0}$ :

$$
\sigma_{c o r r}=\int_{0}^{1} d z_{1} D_{e}\left(z_{1}\right) \int_{0}^{1} d z_{2} D_{e}\left(z_{2}\right) \sigma_{0}(\hat{s})
$$

where $\hat{s}=z_{1} z_{2} s$. Evaluation of this expression, including the first-order diagrams of Fig. 1.3, with the functions

$$
\begin{aligned}
D_{e}(z) & =\frac{\beta}{2}(1-z)^{\frac{\beta}{2}-1} \\
\beta & =\left.\frac{2 \alpha}{\pi}\left(\log \frac{s}{m_{e}^{2}}-1\right)\right|_{s=M_{Z}^{2}}=0.108
\end{aligned}
$$

leads to a $-29 \%$ correction in the peak cross section [6]. This large correction suggests that the second-order diagrams be included; we do so by substituting the expanded function [7]

$$
D_{e}(z)=\frac{\beta}{2}(1-z)^{\frac{\beta}{2}-1}\left(1+\frac{3}{8} \beta\right)-\frac{1}{4} \beta(1+z)
$$

into Eq. 1.22 to obtain the corrected cross section

$$
\sigma=\int_{0}^{1} d x\left[\beta x^{\beta-1}\left(1+\frac{3}{4} \beta\right)-\beta\left(1-\frac{x}{2}\right)\right] \cdot\left[1+\frac{2 \alpha}{\pi}\left(\frac{\pi^{2}}{6}-\frac{1}{4}\right)\right] \sigma_{0}(s(1-x)),
$$

where $x$ is the total fractional radiated energy. A comparison is made in Fig. 1.4 of the uncorrected total $e^{+} e^{-} Z$-pole cross section with the first- and second-order-corrected cross sections. As we will see, the energy shift in the line shape also affects the measurement of electroweak asymmetries, due to the energy dependence from photon- $Z$ interference. 


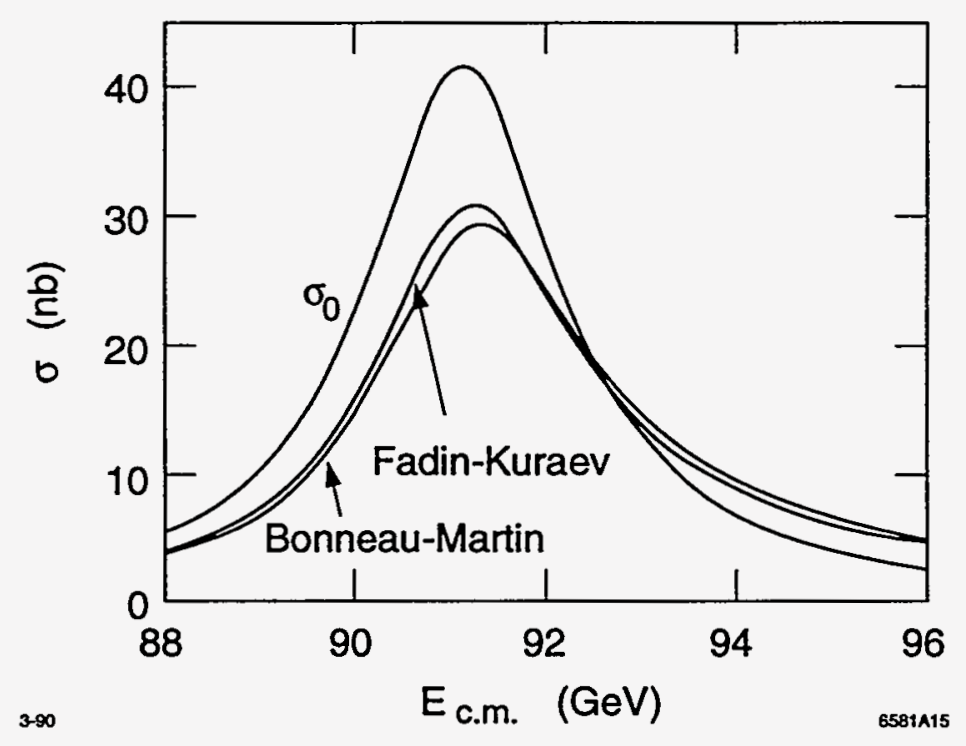

Figure 1.4: Total $e^{+} e^{-} Z$-pole cross section at tree level, with first-order initial-state corrections using the Bonneau-Martin distribution function of Eq. 1.23, and with second-order corrections using the Fadin-Kuraev function of Eq. 1.24.

\subsubsection{Virtual corrections}

While the initial-state QED radiative corrections have a large effect on precision electroweak measurements, the virtual propagator and vertex corrections are more interesting in that these loop diagrams renormalize the measured electroweak parameters. In particular, the electroweak propagator corrections provide information on the still-unknown top and Higgs masses through the interactions of these particles with the gauge bosons.

$\sin ^{2} \theta_{w}:$ definitions and relationships

The study of electroweak radiative corrections inevitably includes a discussion of $\sin ^{2} \theta_{W}$. In order to compare measurements of different electroweak quantities with each other and with Standard Model predictions, it is helpful to relate the measurements to a common parameter. The chosen parameter is the weak mixing angle $\theta_{W}$; unfortunately, a proliferation of $\sin ^{2} \theta_{W}$ definitions in the literature confuses the issue. It is clear from Eqs. 1.5, 1.10, and 1.16 that a number of expressions for $\sin ^{2} \theta_{W}$ are possible using different combinations of the measurable electroweak parameters $\alpha, G_{F}, M_{Z}$, and $M_{W}$. These definitions of the weak mixing angle clearly yield equal values of $\sin ^{2} \theta_{W}$ at lowest order, as they are related to each other through tree-level Standard Model results. Radiative corrections 
to $Z$ production, however, cause the values of these $\sin ^{2} \theta_{W}$ definitions to deviate from the tree-level number and also from each other. For our purposes, it is advantageous to choose a $\sin ^{2} \theta_{W}$ that can be precisely determined at the $Z$ pole, including only corrections due to known physics. In this way, we can interpret a precise measurement of $\sin ^{2} \theta_{W}$ in terms of unknown quantities such as the top and Higgs masses, and, if reconciliation within the Standard Model is not possible, gain understanding of possible extra-Standard-Model effects.

The most common definition of $\sin ^{2} \theta_{W}$ is that due to Sirlin [8], in which the tree-level Standard Model result of Eq. 1.16 is used to define $\sin ^{2} \theta_{W}$ :

$$
\left.\sin ^{2} \theta_{W}\right|_{S} \equiv 1-\frac{M_{W}^{2}}{M_{Z}^{2}}
$$

This definition has the disadvantage that the $W$ mass is not well measured; thus, to achieve an accurate Standard Model prediction of $\left.\sin ^{2} \theta_{W}\right|_{S}$, we must calculate the renormalized $M_{W}$ using $M_{Z}, \alpha$, and $G_{F}$. A clearer and more direct definition is to express $\theta_{W}$ in terms of these three precisely measured electroweak parameters:

$$
\left.\sin 2 \theta_{W}\right|_{Z} \equiv\left[\frac{4 \pi \alpha_{*}\left(M_{Z}^{2}\right)}{\sqrt{2} G_{F} M_{Z}^{2}}\right]^{\frac{1}{2}},
$$

where $\alpha_{*}\left(M_{Z}^{2}\right)$ is the value of the electromagnetic fine-structure constant at the $Z$ mass, renormalized using only the effects of known physics. In this way, the shift of $\left.\sin 2 \theta_{W}\right|_{Z}$ due to corrections from the top quark, Higgs boson, and non-Standard-Model effects can be straightforwardly evaluated, while $\left.\sin 2 \theta_{W}\right|_{Z}$ itself provides an extremely precise reference value of $\sin ^{2} \theta_{W}$. Using the best experimental values of $G_{F}$ and $M_{Z}$ from Table 1.2,

$$
\begin{aligned}
G_{F} & =1.16637(2) \times 10^{-5}(\mathrm{GeV})^{-2} \\
M_{Z} & =91.187(7) \mathrm{GeV}
\end{aligned}
$$

and the current theoretical value for $\alpha$ renormalized to the $Z$ mass [9],

$$
\alpha_{*}^{-1}\left(M_{Z}^{2}\right)=128.80 \pm 0.12
$$

we have

$$
\left.\sin ^{2} \theta_{W}\right|_{z}=0.23136 \pm 0.00031
$$


where the uncertainty is dominated by the theoretical error in computing $\alpha_{*}\left(M_{Z}^{2}\right)$. This uncertainty of 0.00031 in $\left.\sin ^{2} \theta_{W}\right|_{Z}$ represents the current level of understanding of the Standard Model and hence the precision with which it can be tested.

\section{Effective neutral-current interaction}

The vertex factor in the neutral-current interaction of Eq. 1.17 is valid for the tree-level $Z$-exchange process. This process is corrected by the first-order diagrams shown in Figs. 1.5 and 1.6. Fig. 1.5 shows the QED corrections to $e^{+} e^{-} \rightarrow f \bar{f}$ at the $Z$ pole, while Fig. 1.6 shows the electroweak corrections. We discuss the effects of these corrections separately [10].

QED corrections As noted earlier, the tree-level QED vertex factor,

$$
-i e Q_{f} \gamma^{\mu}
$$

leads to a negligible cross section at the $Z$ pole. The effect of the diagrams of Fig. 1.5 is to modify this factor by a correction to the electric coupling and the addition of vector- and axial-vector form factors, yielding

$$
-i \frac{e Q_{f}}{1+\Delta r} \gamma^{\mu}\left[\left(1-F_{A}^{\gamma e} \gamma^{5}\right)+F_{V}^{\gamma e}\right]
$$

The factor $\Delta r$ arises from the photon propagator self-energy, implicitly included in the Fig. 1.5 diagrams, and is of the magnitude

$$
\Delta r \sim-0.03
$$

this shift in the value of $e$ is the source of the renormalized value of $\alpha$ in Eq. 1.26. The typical size of the photonic form factors is

$$
F_{A}^{\gamma e}\left(M_{Z}^{2}\right) \sim F_{V}^{\gamma e}\left(M_{Z}^{2}\right) \sim 10^{-3}
$$

at the $Z$ peak these factors are negligibly small. In addition, the box diagrams of Fig. 1.5, while necessary for gauge invariance, are numerically insignificant.

Electroweak corrections The diagrams of Fig. 1.6 similarly lead to a modification of the vertex factor of Eq. 1.17. Rewriting this tree-level vertex factor in terms of the precisely measured parameters 

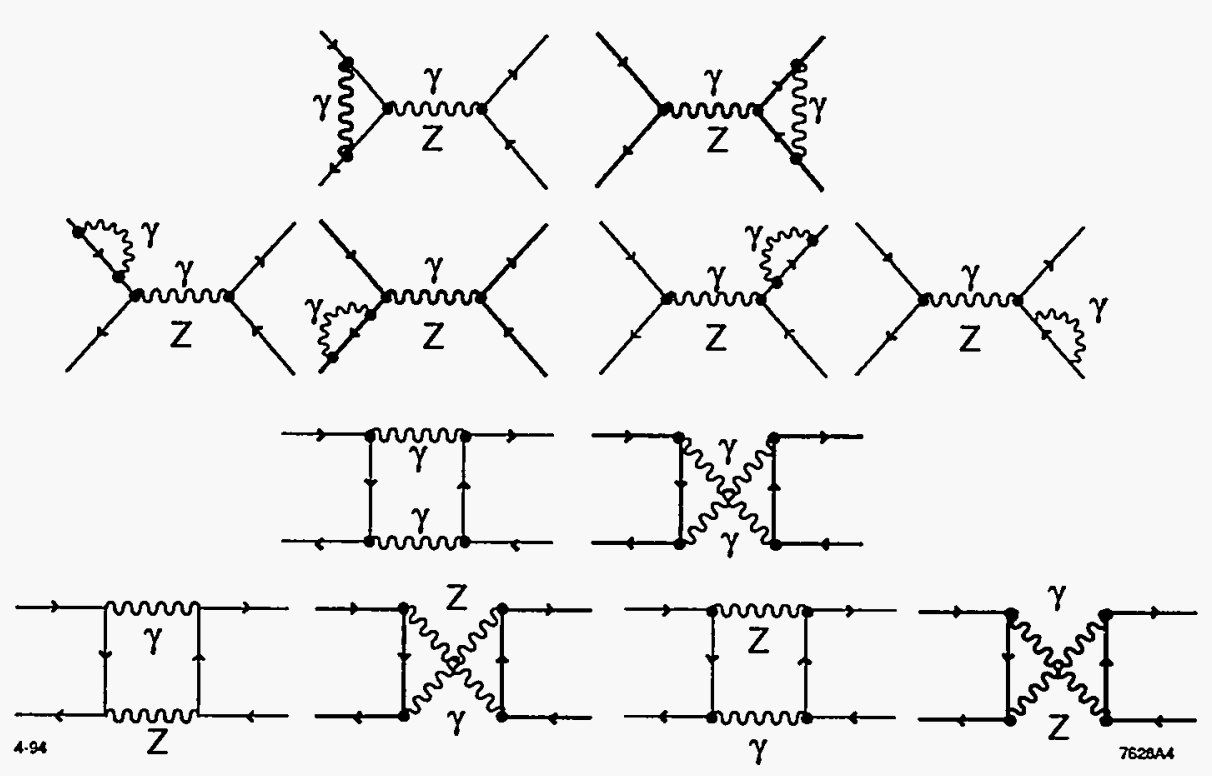

Figure 1.5: First-order virtual QED corrections to the process $e^{+} e^{-} \rightarrow f \bar{f}$ at the $Z$ pole.
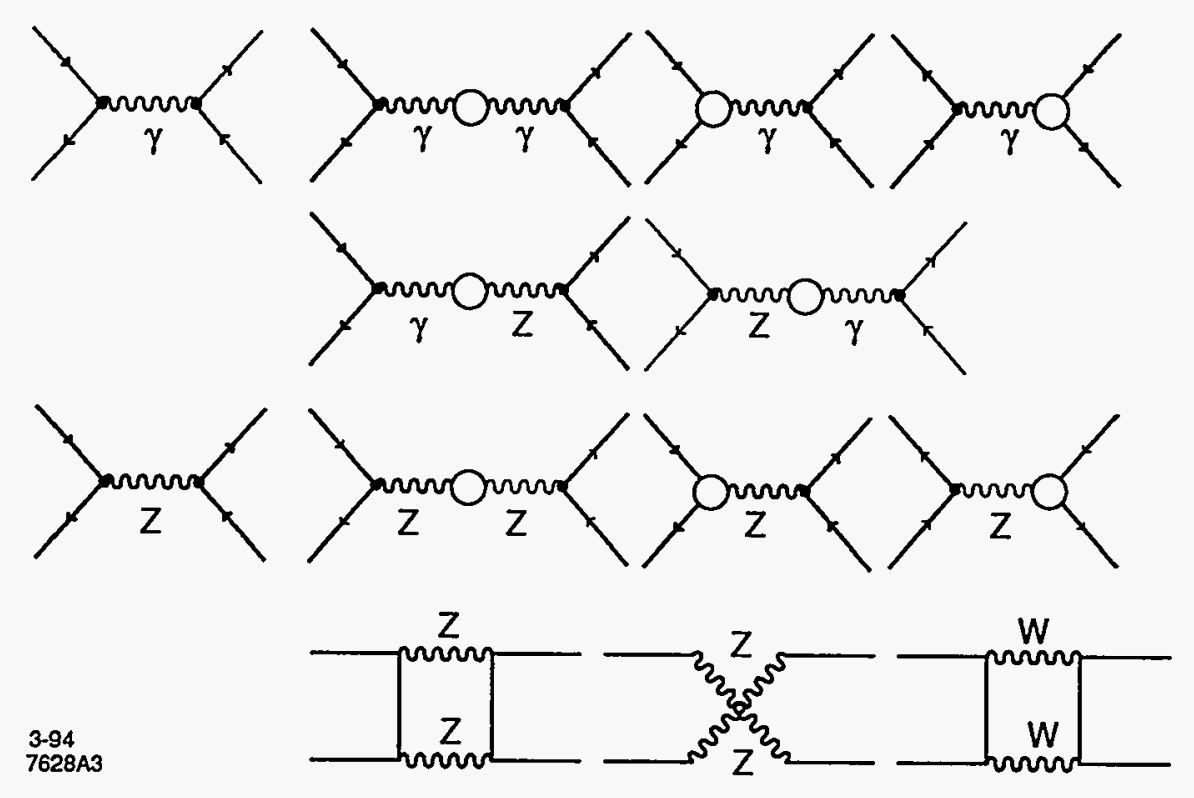

Figure 1.6: First-order virtual electroweak corrections to $e^{+} e^{-} \rightarrow f \bar{f}$. 
$G_{F}$ and $M_{Z}$, we have

$$
-i M_{Z}\left[4 \sqrt{2} G_{F}\right]^{\frac{1}{2}} \gamma^{\mu}\left[\frac{1}{2}\left(1-\gamma^{5}\right) T_{f}^{3}-\left.\sin ^{2} \theta_{W}\right|_{S} Q_{f}\right]
$$

where we have used the Sirlin definition of $\sin ^{2} \theta_{W}$ at tree level. This result is modified in two ways by the electroweak loop corrections. First, the amplitude is renormalized, principally by the propagator loop corrections; second, the vector coupling receives an independent renormalization, due again mainly to the propagator corrections. The form of the vertex factor, however, remains identical to the tree-level result with the addition of the propagator and vector form factors $\rho_{f}$ and $\kappa_{f}:$

$$
-i M_{Z}\left[4 \sqrt{2} G_{F} \rho_{f}\right]^{\frac{1}{2}} \gamma^{\mu}\left[\frac{1}{2}\left(1-\gamma^{5}\right) T_{f}^{3}-\left.\kappa_{f} \sin ^{2} \theta_{W}\right|_{S} Q_{f}\right]
$$

This convenient factoring of the corrections is valid only with the neglect of the box diagrams, which again remain small for the electroweak corrections. The form factors $\rho_{f}$ and $\kappa_{f}$ can be separated into a "universal" part, independent of fermion species, and a "non-universal" part, which depends on the species:

$$
\begin{aligned}
& \rho_{f}=1+(\Delta \rho)_{u n i v}+(\Delta \rho)_{n o n-u n i v} \\
& \kappa_{f}=1+(\Delta \kappa)_{u n i v}+(\Delta \kappa)_{n o n-u n i v}
\end{aligned}
$$

The universal parts, which are due to the propagator corrections, dominate the non-universal parts, which arise from the vertex corrections and the fermion self-energies in the external lines. The leading terms of the universal parts of $\rho$ and $\kappa$ are given by

$$
\begin{aligned}
(\Delta \rho)_{\text {univ }} & \sim \Delta \rho \\
(\Delta \kappa)_{\text {univ }} & \sim \frac{\left.\cos ^{2} \theta_{W}\right|_{S}}{\left.\sin ^{2} \theta_{W}\right|_{S}} \Delta \rho
\end{aligned}
$$

where

$$
\Delta \rho=\frac{3 \alpha}{\left.\left.16 \pi \sin ^{2} \theta_{W}\right|_{S} \cos ^{2} \theta_{W}\right|_{S}} \frac{m_{t}^{2}}{M_{Z}^{2}}
$$

and $\left.\sin ^{2} \theta_{W}\right|_{S}$ is the Sirlin definition of $\sin ^{2} \theta_{W}$ with $\left.\cos ^{2} \theta_{W}\right|_{S}=1-\left.\sin ^{2} \theta_{W}\right|_{S}$. Due to the preservation of the form of the tree-level vertex factor, Eq. 1.27 suggests an "effective" (renormalized) $\sin ^{2} \theta_{W}$

$$
\left.\left.\sin ^{2} \theta_{W}^{\text {eff }} \equiv \kappa_{f} \sin ^{2} \theta_{W}\right|_{S} \equiv \sin ^{2} \theta_{W}\right|_{u n i v}+\left.(\Delta \kappa)_{\text {ron-univ }} \sin ^{2} \theta_{W}\right|_{S}
$$


so that we may rewrite Eq. 1.27 as

$$
-i M_{Z}\left[4 \sqrt{2} G_{F} \rho_{f}\right]^{\frac{1}{2}} \gamma^{\mu}\left[\frac{1}{2}\left(1-\gamma^{5}\right) T_{f}^{3}-\sin ^{2} \theta_{W f}^{\mathrm{eff}} Q_{f}\right]
$$

In Eq. 1.28 we have also introduced a "universal" weak mixing angle for all fermion species given in leading order by

$$
\left.\left.\sin ^{2} \theta_{W}\right|_{\text {univ }} \equiv \sin ^{2} \theta_{W}\right|_{S}+\left.\cos ^{2} \theta_{W}\right|_{S} \Delta \rho
$$

The universal weak mixing angle is equivalent to another popular $\sin ^{2} \theta_{W}$, the $s_{*}^{2}$ of Kennedy and Lynn [10]. It is clear from the preceding, however, that $\left.\sin ^{2} \theta_{W}\right|_{u n i v}$ does not include the renormalization of $\sin ^{2} \theta_{W}$ due to vertex corrections; these corrections are typically of order $\Delta \sin ^{2} \theta_{W} \sim 0.0004$, and so are necessary only in comparing with results of high-precision electroweak measurements.

Several programs exist [11] to compute the effects of initial-state radiation and first-order QED and electroweak loop diagrams on $e^{+} e^{-}$annihilation at the $Z$ pole. A calculation based on a modified version [12] of the program ZSHAPE yields the top- and Higgs-mass dependence of $\sin ^{2} \theta_{W}^{\mathrm{eff}}\left(M_{Z}^{2}\right)$ shown in Fig. 1.7. For this plot, $\sin ^{2} \theta_{W}^{\text {eff }}\left(M_{Z}^{2}\right)$ has been averaged over fermion species weighted by their $Z$ partial width. Using the same program, we can calculate $M_{W}$ and hence find $\left.\sin ^{2} \theta_{W}\right|_{S}$ as a function of top and Higgs mass; this graph is shown in Fig. 1.8. We can use these graphs to translate between the two definitions of $\sin ^{2} \theta_{W}$ at any value of top and Higgs mass.

\subsection{Electroweak Asymmetries}

The structure of the polarized differential $Z$ cross section allows several tests of the couplings of the initial- and final-state fermions to the $Z$. An advantageous technique in precision electroweak physics is to avoid the systematic error inherent in measuring absolute cross sections by forming ratios of cross sections in which different initial- or final-state characteristics, such as polarization or decay angle, have been selected. Several such asymmetries provide important measurements of electroweak couplings.

\subsubsection{Forward-backward asymmetry}

Forward-backward asymmetries have long been employed to observe $\gamma-Z$ interference at energies below the $Z$ pole $[13,14]$. We define the forward-backward asymmetry for the process $e^{+} e^{-} \rightarrow f \bar{f}$ 


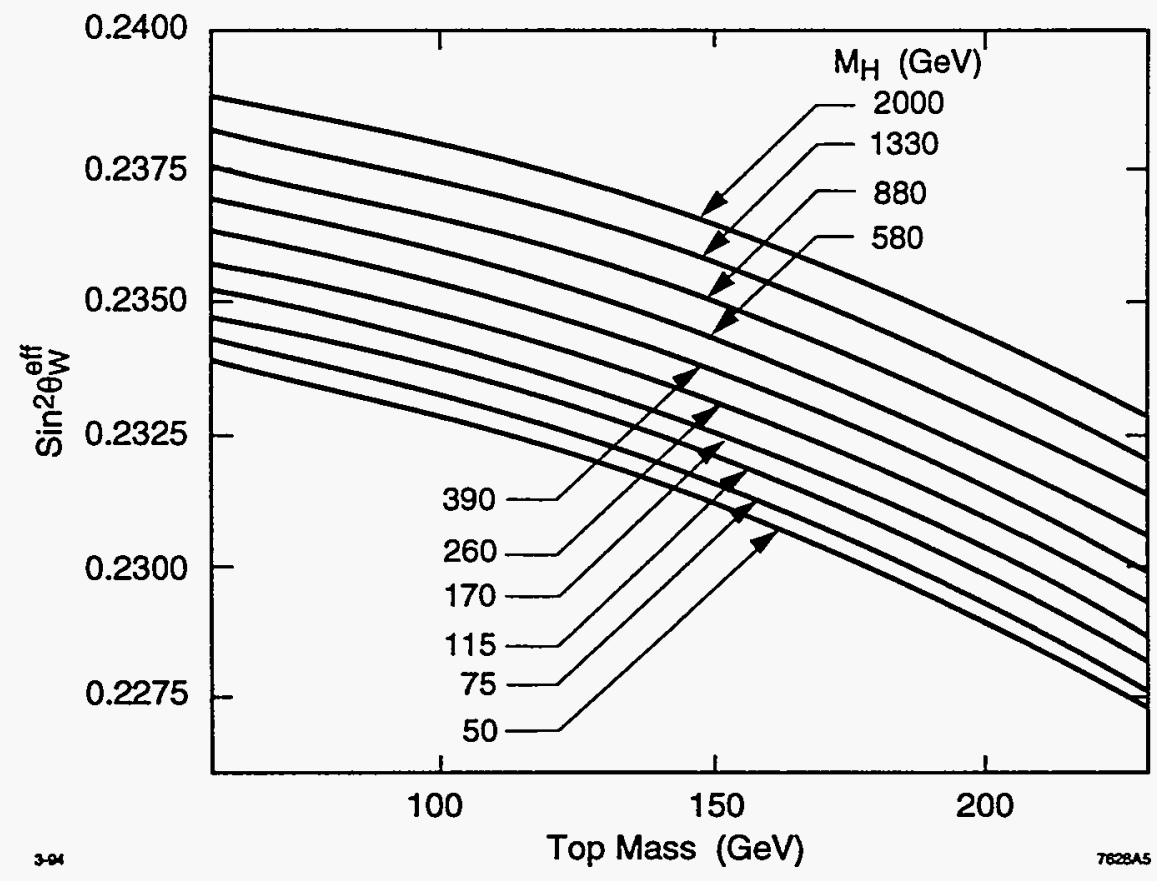

Figure 1.7: Dependence of $\sin ^{2} \theta_{W}^{\mathrm{eff}}\left(M_{Z}^{2}\right)$ on top and Higgs masses.

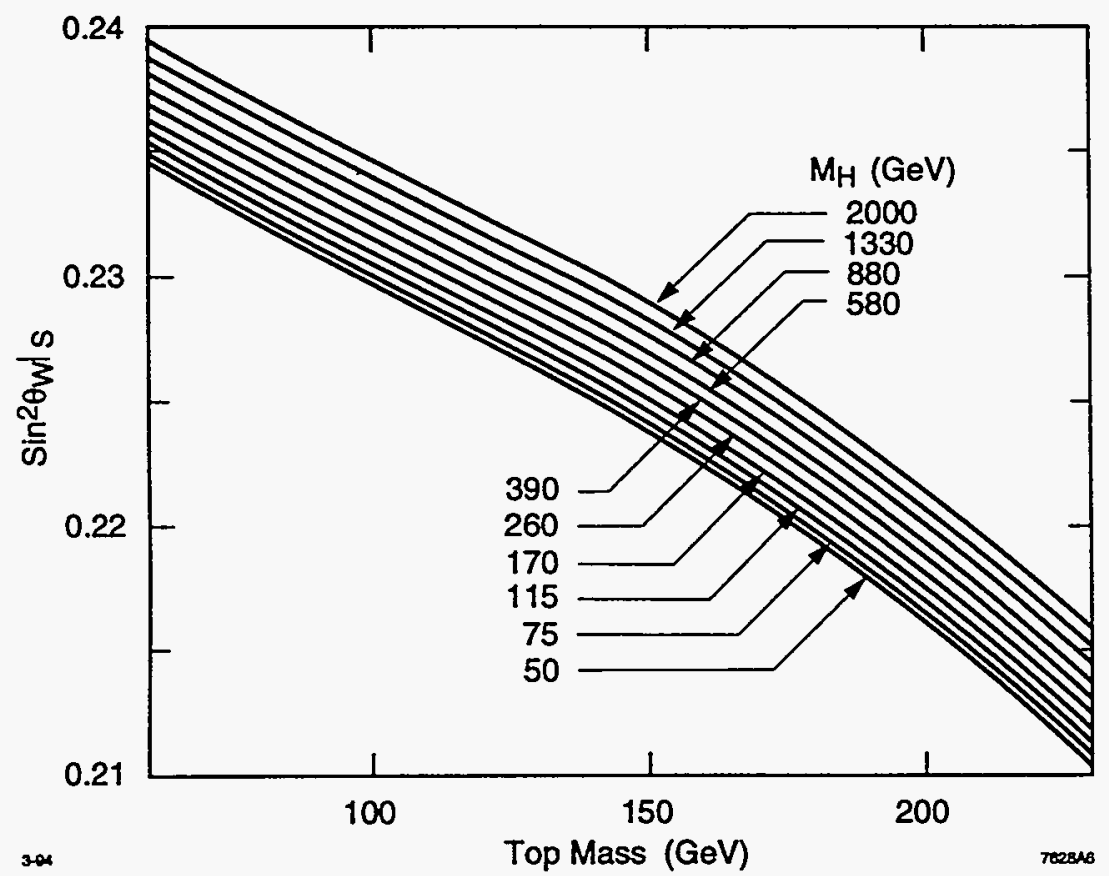

Figure 1.8: Dependence of $\left.\sin ^{2} \theta_{W}\right|_{S}$ on top and Higgs masses. 
as

$$
A_{F B}^{f} \equiv \frac{\int_{0}^{c_{0}} d c \frac{d \sigma_{f}}{d c}-\int_{-c_{0}}^{0} d c \frac{d \sigma_{f}}{d c}}{\int_{-c_{0}}^{c_{0}} d c \frac{d \sigma_{f}}{d c}},
$$

where $c_{0}$ is a detector integration limit. Substitution of the Z-peak cross section of Eq. 1.20 yields

$$
A_{F B}^{f}=\frac{4 c_{0}}{3+c_{0}^{2}} \cdot \frac{3 c_{A}^{e} c_{V}^{e} c_{A}^{f} c_{V}^{f}}{\left(c_{A}^{e}+c_{V}^{e}\right)\left(c_{A}^{f^{2}}+c_{V}^{f^{2}}\right)}
$$

making the useful notational substitution

$$
A_{L R}^{f} \equiv \frac{2 c_{A}^{f} c_{V}^{f}}{c_{A}^{f^{2}}+c_{V}^{f^{2}}}
$$

to be explained in the next section, we have

$$
A_{F B}^{f}=\frac{3}{4} \cdot \frac{4 c_{0}}{3+c_{0}^{2}} \cdot A_{L R}^{e} \cdot A_{L R}^{f}
$$

The forward-backward asymmetry of Eqs. 1.31 and 1.32 has several important features. The appearance of the final-state fermion couplings requires that the measurement be made with an identifiable decay channel, usually chosen to be muons or $b$ quarks. The relative experimental ease of tagging leptons for this measurement is offset by the small value of $A_{F B}^{l}$ of $\sim 2 \%$, compared with $\sim 11 \%$ for $A_{F B}^{b}$. In addition, the sensitivity of $A_{F B}$ to the weak mixing angle is much smaller for leptons than for $b$ quarks: we have, at tree level,

$$
\begin{aligned}
& \frac{d A_{F B}^{b}}{d \sin ^{2} \theta_{W}}=-5.58 \\
& \frac{d A_{F B}^{l}}{d \sin ^{2} \theta_{W}}=-1.87 .
\end{aligned}
$$

\subsection{2 $\tau$-polarization asymmetry}

The ability to determine the $Z$ spin direction allows the possibility of forming a polarization asymmetry with the $Z$ sample. With longitudinally unpolarized colliding beams, this spin discrimination can still be made by examining the $Z$ final state. Defining the final-state polarization of a $Z$ decay at a particular fermionic polar angle as

$$
\mathcal{P}_{f}(\cos \theta) \equiv \frac{\frac{d \sigma}{d \Omega}\left(f_{L}\right)-\frac{d \sigma}{d \Omega}\left(f_{R}\right)}{\frac{d \sigma}{d \Omega}\left(f_{L}\right)+\frac{d \sigma}{d \Omega}\left(f_{R}\right)}
$$


where $f_{L}$ and $f_{R}$ denote left- and right-handed fermions, we substitute the unpolarized part of the $Z$ cross section from Eq. 1.20 to obtain

$$
\mathcal{P}_{f}(\cos \theta)=\frac{2 A_{L R}^{e} \cos \theta+A_{L R}^{f}\left(1+\cos ^{2} \theta\right)}{\left(1+\cos ^{2} \theta\right)+2 A_{L R}^{e} A_{L R}^{f} \cos \theta}
$$

Integration of this expression over symmetric $\cos \theta$ limits removes the initial-state couplings and leaves the simple result

$$
\left\langle\mathcal{P}_{f}\right\rangle=A_{L R}^{f}
$$

The final-state polarization tag is made with the $\tau$ lepton, which decays via a $V-A$ current into a $\tau$-polarization-dependent angular distribution.

The average $\tau$-polarization $\left\langle P_{\tau}\right\rangle$ is larger than the forward-backward asymmetries, at $\sim 14 \%$; it is insensitive to initial-state radiative effects; and it is very sensitive to electroweak radiative corrections from the top quark and Higgs boson and to the weak mixing angle:

$$
\frac{d\left\langle\mathcal{P}_{\tau}\right\rangle}{d \sin ^{2} \theta_{W}}=-7.84
$$

The sample size is limited, however, by the small $\tau$ partial width and selection efficiency to $\sim 2 \%$ of the $Z$ sample.

\subsection{The Left-Right Asymmetry}

The availability of longitudinal beam polarization permits a more direct probe of the structure of electroweak currents. By preparing the initial spin state of the electrons, it is possible to make a simpler and more statistically powerful measurement of the $e^{-}-Z$ coupling.

\subsubsection{Properties of $A_{L R}$}

The left-right asymmetry $A_{L R}$ for $Z$ decays to a particular final-state fermion pair $f \bar{f}$ is defined as

$$
A_{L R} \equiv \frac{\sigma_{E}\left(e_{R}^{+} e_{L}^{-} \rightarrow Z \rightarrow f \bar{f}\right)-\sigma_{E}\left(e_{L}^{+} e_{R}^{-} \rightarrow Z \rightarrow f \bar{f}\right)}{\sigma_{E}\left(e_{R}^{+} e_{L}^{-} \rightarrow Z \rightarrow f \bar{f}\right)+\sigma_{E}\left(e_{L}^{+} e_{R}^{-} \rightarrow Z \rightarrow f \bar{f}\right)}
$$

where $\sigma_{E}$ is the total $Z$ cross section at center-of-mass energy $E$. We can evaluate $A_{L R}$ at tree level on the $Z$ pole by integrating Eq. 1.20 and substituting into the $A_{L R}$ definition, regaining the form 
of Eq. 1.31 for $f=e^{-}$:

$$
A_{L R}=\frac{2 c_{V}^{e} c_{A}^{e}}{c_{V}^{e^{2}}+c_{A}^{e}}
$$

Thus, assuming lepton universality, the left-right asymmetry is equal to the average $\tau$-polarization $\left\langle P_{\tau}\right\rangle$. We see that we are justified in omitting reference to the final-state fermion in our notation $A_{L R}$ : at tree level, there is no final-state-coupling dependence in $A_{L R}$. This feature leaves the asymmetry with only a single power of $c_{V}^{e}$ in the numerator, giving $A_{L R}$ a magnitude of $\sim 14 \%$; more importantly, the lack of final-state dependence allows the use of all $Z$ decays for the $A_{L R}$ measurement, save Bhabha events, which can proceed via $t$-channel photon exchange. In addition, as will be developed in Chapter 5, the form of Eq. 1.34 is independent of the integration limit chosen for Eq. 1.20; in fact, given equal local efficiencies for fermionic and antifermionic detection, $A_{L R}$ is completely independent of detector acceptance. If symmetric integration limits are chosen, the result of Eq. 1.34 is still obtained when the $\left(1+c^{2}\right)$ - and $c$-dependent terms of Eq. 1.20 are replaced with arbitrary symmetric and antisymmetric functions, implying that $A_{L R}$ is independent of final-state strong interactions [15]. The left-right asymmetry has the same high sensitivity to $\sin ^{2} \theta_{W}$ as $\left\langle P_{\tau}\right\rangle$ :

$$
\frac{d A_{L R}}{d \sin ^{2} \theta_{W}}=-7.84
$$

These qualities allow $A_{L R}$ to provide a precise test of the Standard Model with a modest $Z$ event sample.

\subsubsection{Radiative corrections to $A_{\text {LR }}$}

The inclusion of higher-order corrections to $A_{L R}$ follows directly from the earlier development of radiative corrections to $Z$ production. We must consider the effects of initial-state radiation and virtual QED and electroweak corrections.

\section{Initial-state radiation}

To investigate the influence of initial-state radiation on $A_{L R}$, we insert the cross-section correction of Eq. 1.25 and the photon- $Z$ interference terms of Eq. 1.21 into the $e^{+} e^{-} \rightarrow Z$ cross section. The results of calculating $A_{L R}$ with this corrected cross section are shown in Fig. 1.9. We see that the effect on $A_{L R}$ is to shift the energy dependence by a few hundred MeV. The weak energy dependence of $A_{L R}$ keeps the initial-state-radiation corrections small. 


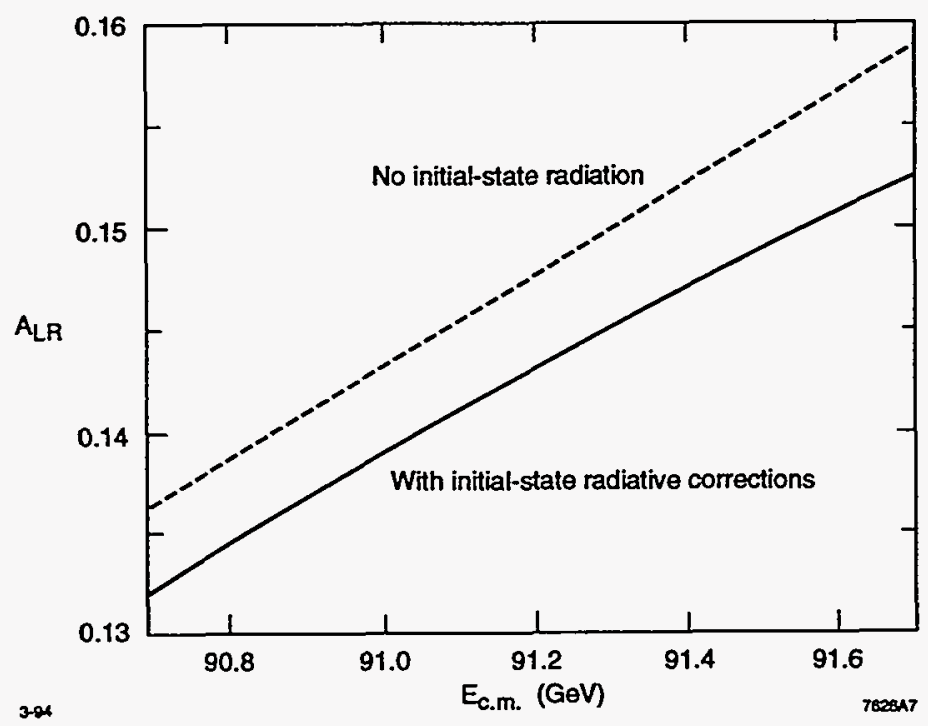

Figure 1.9: $A_{L R}$ as a function of center-of-mass energy with and without initial-state radiation.

\section{Virtual corrections}

As we saw in the $\sin ^{2} \theta_{W}$ discussion, virtual loop corrections can be completely incorporated into the cross section by the vertex factor renormalization of Eq. 1.27. Therefore, the effect on $A_{L R}$ of these corrections is included by calculating $A_{L R}$ using the cross section with the renormalized vertex factor. Using Eqs. 1.19 and 1.34, we rewrite $A_{L R}$ in terms of the zero-order $\sin ^{2} \theta_{W}$ as

$$
A_{L R}^{0}=\frac{2\left(1-4 \sin ^{2} \theta_{W}\right)}{1+\left(1-4 \sin ^{2} \theta_{W}\right)^{2}} ;
$$

use of the renormalized vertex factor yields the corrected $A_{L R}$

$$
A_{L R}=\frac{2\left[1-4 \sin ^{2} \theta_{W}^{\mathrm{eff}}\left(M_{Z}^{2}\right)\right]}{1+\left[1-4 \sin ^{2} \theta_{W}^{\mathrm{eff}}\left(M_{Z}^{2}\right)\right]^{2}} .
$$

We see that the vertex amplitude renormalization factor $\rho$ cancels in the asymmetry and the effect of the loop corrections on $A_{L R}$ is simply to substitute the effective weak mixing angle for the tree-level value. 


\section{Corrected $A_{L R}$ result}

Using the virtual loop corrections to $\sin ^{2} \theta_{W}^{\text {eff }}$ of Fig. 1.7 and the initial-state-radiation correction from Fig. 1.9, we can now plot the fully corrected, experimentally measurable value of $A_{L R}$ as a function of top and Higgs mass; this plot is shown in Fig. 1.10. From this graph, we see that $A_{L R}$ has some

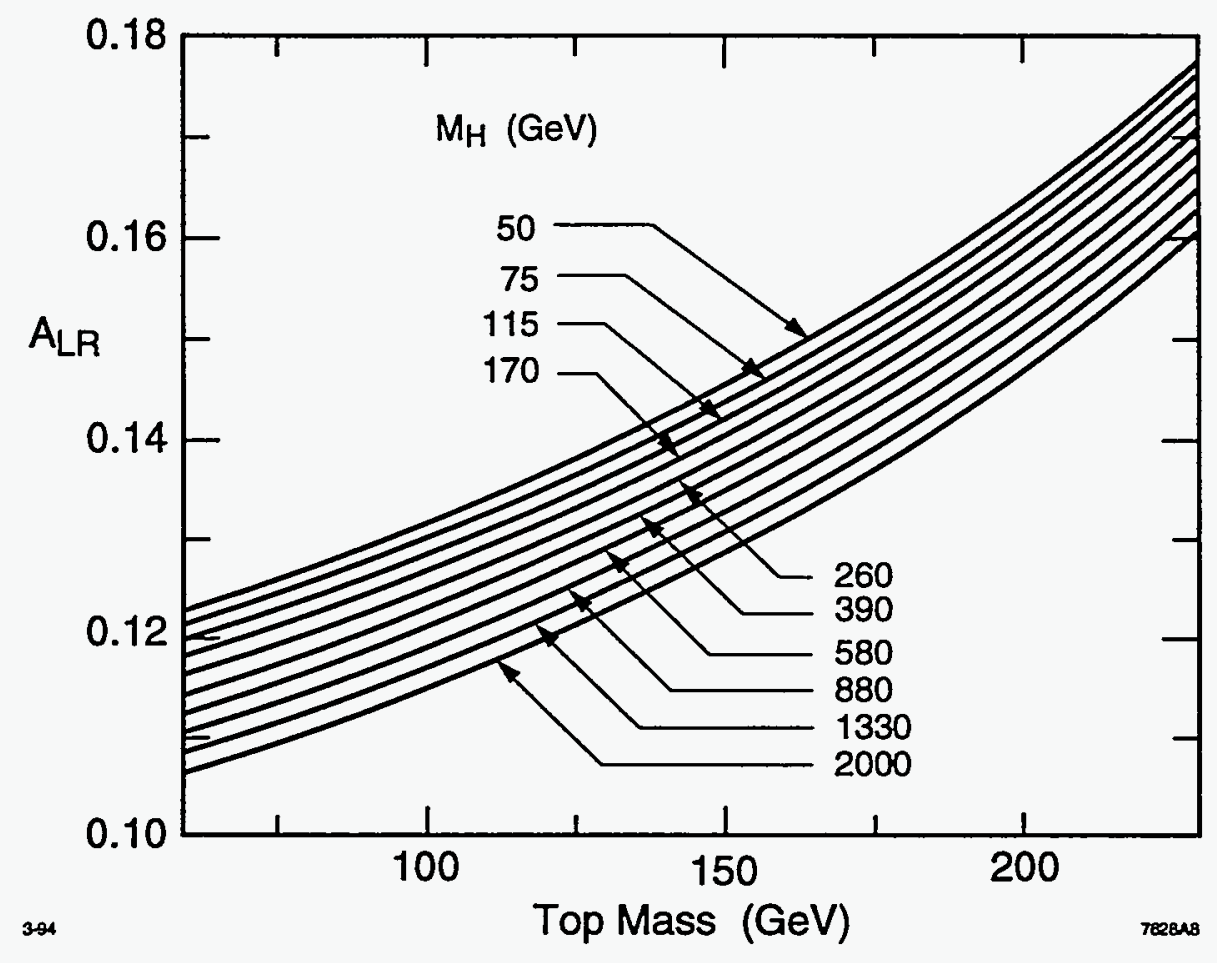

Figure 1.10: The fully corrected $A_{L R}$ as a function of top and Higgs mass.

sensitivity to the unknown masses; however, this steep dependence allows a large range of possible Standard Model $A_{L R}$ values. Thus, a measurement of $A_{L R}$ serves to constrain a combination of top and Higgs masses but cannot by itself determine either unknown parameter or precisely test the Standard Model. $A_{L R}$ must be compared with other precision electroweak measurements, such as the $Z$ width, with different trajectories in the top-Higgs mass plane. The intersection of these curves determines the unknown masses within the Standard Model, and, by comparison with other mass measurements, provides tests of the Standard Model and alternative theories [16]. 


\subsection{Measuring $A_{L R}$}

A major advantage of $A_{L R}$ over competing precision electroweak measurements is its relative experimental ease. At heart, $A_{L R}$ is a $Z$-counting experiment in which the beam polarization is measured and bunch helicity recorded for each event. We develop here the fundamental elements of the measurement.

\subsubsection{Error equation}

From Eq. 1.33, it is clear that with complete electron-beam polarization and equal left- and righthanded luminosities, $A_{L R}$ is given simply by the measured $Z$ asymmetry:

$$
\left.A_{L R}\right|_{\mathcal{P}=1}=\frac{N_{L}-N_{R}}{N_{L}+N_{R}} \equiv A_{m}
$$

where $N_{L}$ and $N_{R}$ are the number of $Z$ s made with left- and right-handed electron beam. More generally, in the case of beam with partial polarization $\mathcal{P}^{e}$, the relationship between $A_{L R}$ and $A_{m}$ becomes

$$
A_{L R}=\frac{1}{p e} A_{m} .
$$

This result immediately yields the $A_{L R}$ error equation

$$
\delta A_{L R}=\left[\frac{1}{\mathcal{P}^{2} N}+A_{L R}^{2}\left(\frac{\delta \mathcal{P}}{\mathcal{P}}\right)^{2}\right]^{\frac{1}{2}},
$$

where $N$ is the total number of $Z s$ in the sample and where we have assumed $\left(\mathcal{P}^{e} A_{L R}\right)^{2} \ll 1$. The equation contains a statistical part, with the expected $N^{-1 / 2}$ dependence modulated by a factor $\mathcal{P}^{-1}$, and a systematic part related to the measurement precision of the beam polarization. Thus, we see that the measurement benefits by a large $Z$ sample, but also by high and well-measured polarization. The systematic part includes a factor of $A_{L R}^{2} \sim 2 \%$, ensuring that this term will remain small compared with the statistical error even for a precision experiment. The $A_{L R}$ uncertainty is plotted in Fig. 1.11 vs. $Z$-sample size for various values of polarization and $\delta \mathcal{P} / \mathcal{P}$.

\subsubsection{Polarization measurement}

The measurement of $A_{L R}$ requires knowing the polarization and helicity of the electron bunch that creates each $Z$. Unfortunately, no polarimetry device can measure with sufficient statistical accuracy 


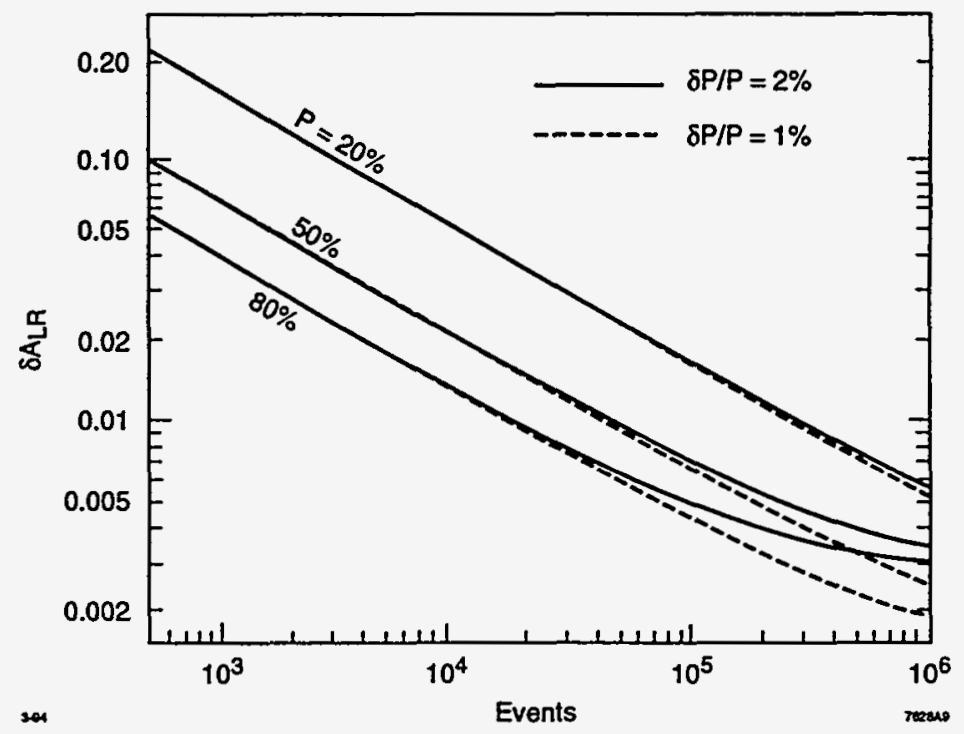

Figure 1.11: Error in $A_{L R}$ measurement as a function of $Z$ sample, polarization, and polarization measurement error.

the polarization of a single electron bunch. Therefore, we measure the polarization continually in several-minute runs and rely on the assumption that there is no short-term relationship between luminosity and beam polarization.

Eq. 1.36 provides the relationship between $A_{L R}$ and the measured $Z$ asymmetry via the beam polarization. In practice, the polarization has some variation over the length of the experimental program. Therefore, we require a version of Eq. 1.36 that allows for a time-dependent beam polarization. It is easily shown that if the beam polarization does not fluctuate greatly, we have the result

$$
A_{L R}=\frac{A_{m}}{\overline{\mathcal{P}^{e}}}
$$

where $\overline{\mathcal{P}^{e}}$ is the luminosity-weighted average beam polarization over the run:

$$
\overline{\mathcal{P}^{e}} \equiv \frac{\int d t \mathcal{L}(t) \mathcal{P}(t)}{\int d t \mathcal{L}(t)}
$$

Other expressions are possible to increase statistical power in combining blocks of data with greatly different average polarizations, e.g., in combining runs made with different polarized sources [17]. Using the $Z$ count to measure luminosity, and assuming a negligible left-right luminosity asymmetry, we can rewrite Eq. 1.37 as a sum over the $N Z$ events, where $\mathcal{P}_{i}$ is the polarization measured in the 
polarimeter run coincident with the creation of the $i^{\text {th }} Z$ :

$$
\overline{\mathcal{P}^{e}}=\frac{1}{N} \sum_{i=1}^{N} \mathcal{P}_{i}
$$

It is clear from the form of Eq. 1.38 that the value of $\overline{\mathcal{P}^{e}}$ is independent of the chosen length of the polarimeter run. Shorter polarimeter runs, however, increase the statistical uncertainty of the measured polarization, and hence widen the resulting $\mathcal{P}_{i}$ distribution. In any case, the error in the mean of the distribution will be entirely negligible compared with the overall polarization systematic error. Experimentally, it is possible to obtain a statistical uncertainty of $\delta \mathcal{P} \sim 1 \%$ in a three-minute run, while $\overline{\mathcal{P}^{e}}$ is found by averaging over the entire physics run of several months duration. 


\section{Chapter 2}

\section{Apparatus}

The SLAC Linear Collider (SLC) is an $e^{+} e^{-}$machine designed to produce the $Z$ boson copiously in a low-background environment. The SLC was completed in 1987 and began producing $Z$ bosons in 1989 with the Mark II detector, which accumulated 850 events before its retirement in 1990 . The new SLD detector replaced the Mark II in 1991 and began its run with fourfold-enhanced luminosity and greater SLC reliability. For the 1992 run, the data from which are used in this measurement, the SLC began creating $Z$ bosons with a longitudinally polarized electron beam [18, 19], a capability unique among $Z$-producing machines. This advance, along with another fourfold increase in luminosity, allowed the SLC/SLD to begin making precision electroweak measurements. The integrated luminosity for the 1992 run in numbers of $Z s$ is shown in Fig. 2.1.

\subsection{The Polarized SLC}

Even without polarization, the SLC [20] is a challenging and innovative design. It is the first and only linear $e^{+} e^{-}$collider in the world, built upon the foundation of the SLAC linear accelerator (linac). The polarized SLC, shown in Fig. 2.2, represents a further technological breakthrough.

Polarized electron bunches are created by photoemission from a gallium arsenide cathode and are injected into the linac. A spin- rotation solenoid then brings the electron spins into vertical orientation for storage in the north damping ring for one machine cycle, after which two more spin rotators reorient the spin for transport down the linac. A Møller polarimeter at the end of the linac can be inserted diagnostically to monitor polarization before entry into the north arc. The polarized electrons then travel to the interaction point, collide with an unpolarized positron bunch, 


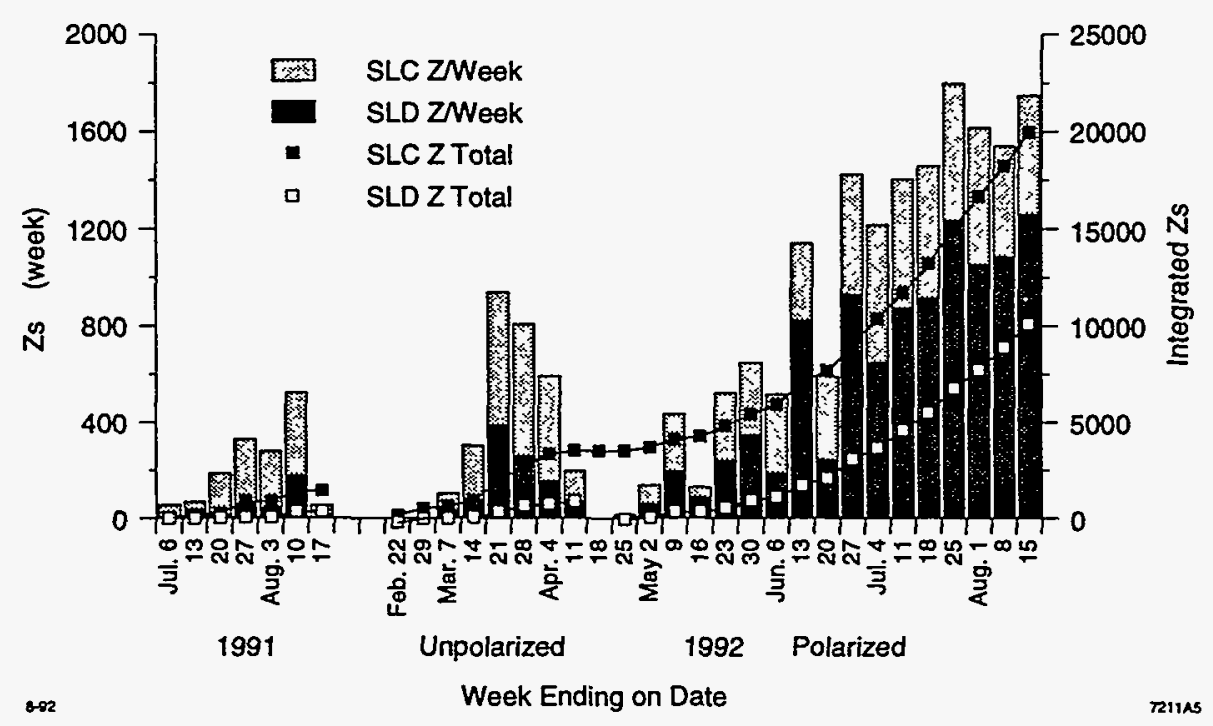

Figure 2.1: $Z$ count as a function of time in the 1991-1992 SLD run. Polarized running began at the end of April 1992.

and pass to a Compton polarimeter, where the beam polarization is analyzed. Both the electron and positron bunches traverse precision energy spectrometers before they are kicked out of the beampipe to dumps.

\subsubsection{Polarized source}

The phenomenon of polarized electron emission from a gallium arsenide crystal was discovered in 1976 [21] and has since been exploited for use in accelerator experiments. The system used at SLC is a modified form of the polarized source for the groundbreaking parity-violation experiment at SLAC in 1978 [22]. The SLC polarized source layout is shown in Fig. 2.3.

A flashlamp-pumped dye laser operating at $120 \mathrm{~Hz}$ and $715 \mathrm{~nm}$, in conjunction with a Pockelscell-based Laser Pulse Chopper, make two $2 \mathrm{~ns}, 25 \mu \mathrm{J}$ light pulses separated by $60 \mathrm{~ns}$. The Pockels cell, a high-voltage electro-optic device whose birefringence is voltage-dependent, is driven by a custom source capable of producing $3 \mathrm{kV}, 2$ ns pulses. The first light pulse is used to make electrons for $e^{+} e^{-}$collisions; the second produces an electron bunch that makes positrons for the next machine cycle. The pulses pass through a Bunch Intensity Controller to stabilize long-term light intensity on the photocathode. At this point the light is linearly polarized, and it now meets the Circular 


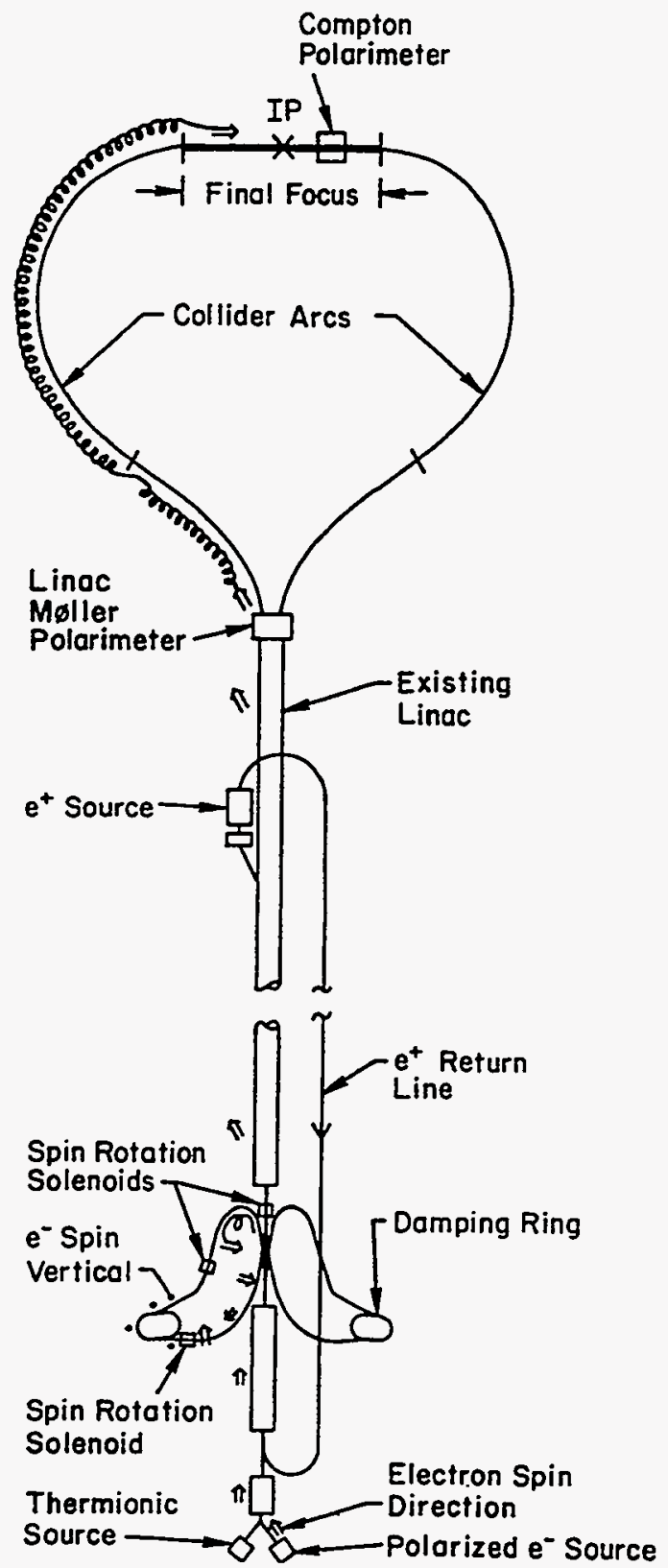

POLARIZATION IN THE OVERALL SLC LAYOUT

$10-86$

557114

Figure 2.2: The polarized SLC. The electron spin direction is indicated by the double arrow. 


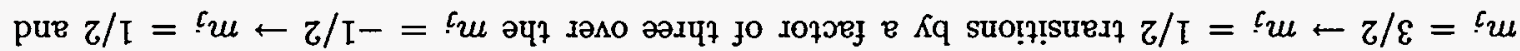

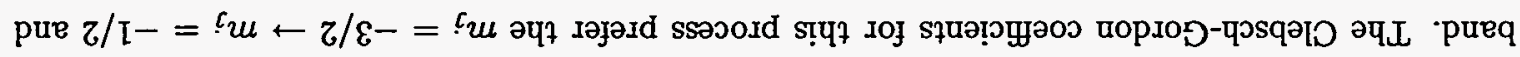

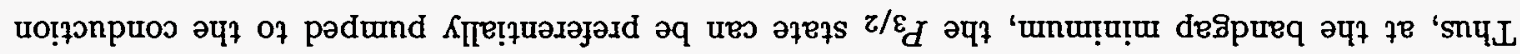

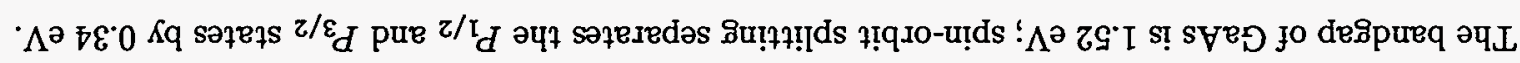
'7. $Z$ '

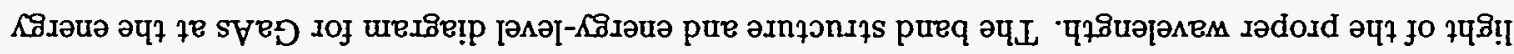

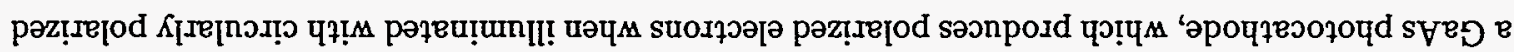

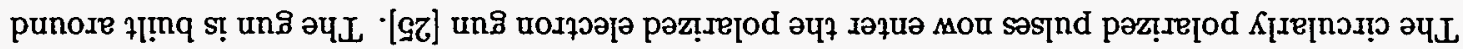

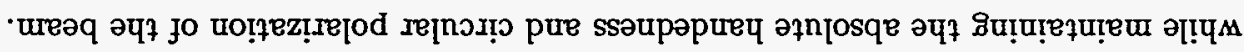

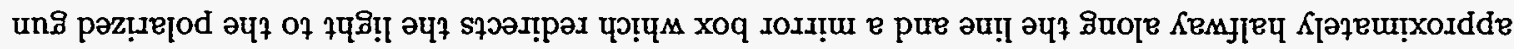

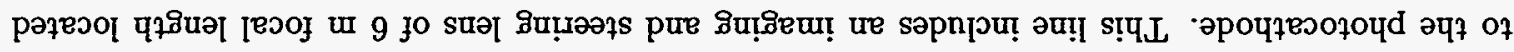

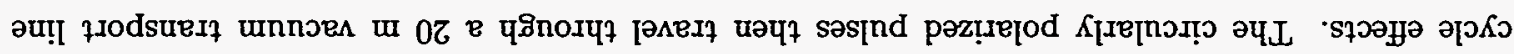

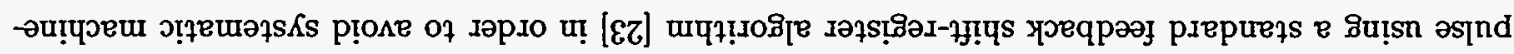

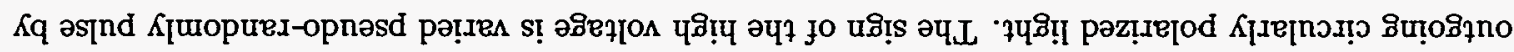

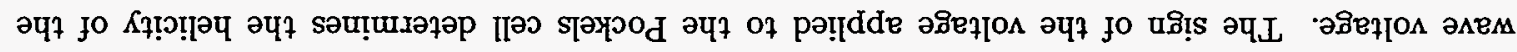

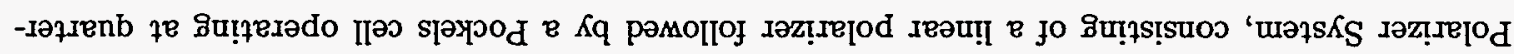

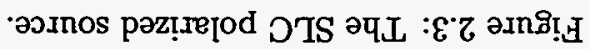

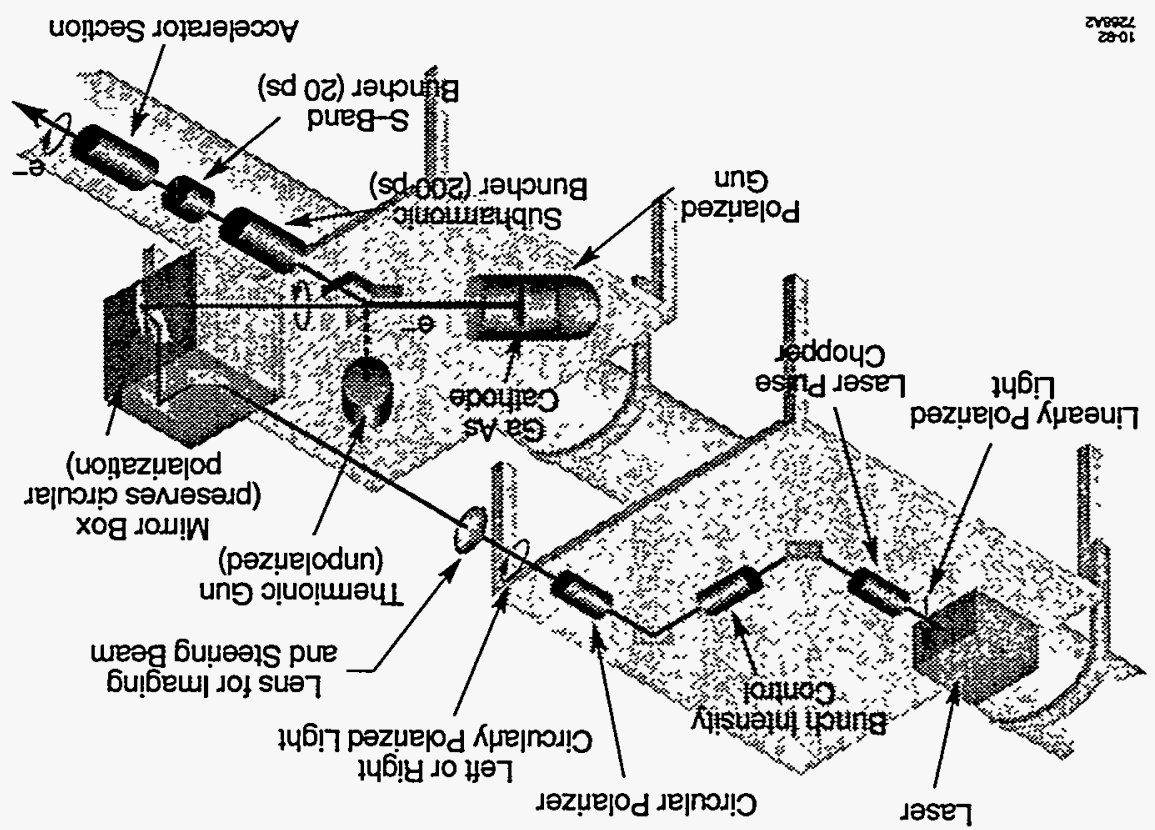

$0 \varepsilon$ 


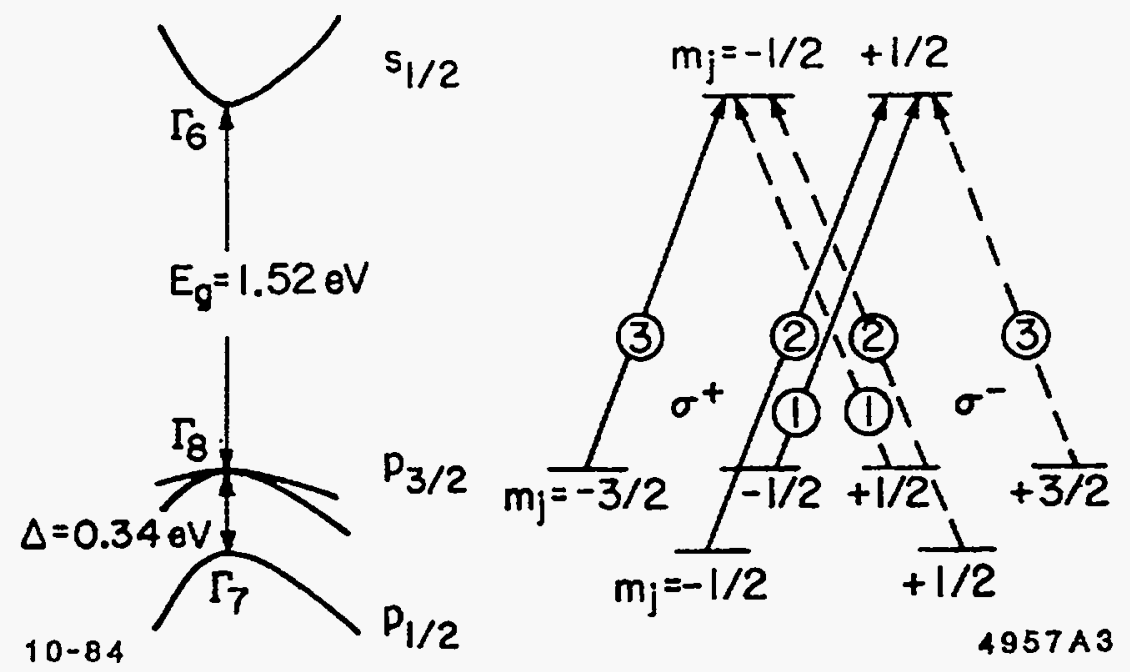

Figure 2.4: The band structure and energy-level diagram for GaAs. Allowed transitions for the absorption of right (left) circularly polarized photons are shown as solid (dashed) arrows. The circled numbers indicate the relative transition rates.

$m_{j}=1 / 2 \rightarrow m_{j}=-1 / 2$ transitions. The maximum electron polarization from this source is then ${ }^{1}$

$$
\mathcal{P}_{\text {max }}=\frac{3-1}{3+1}=50 \%
$$

By treating the surface of the photocathode with cesium, the work function of the GaAs can be brought to zero or even made negative. The polarized electron gun itself (Fig. 2.5) is a diode design operating at $120 \mathrm{kV}$. A retractable effusion-cell cesium dispenser is used periodically to deposit a layer of $\mathrm{CsF}$ on the photocathode during the run. The interior of the gun must be held in ultra-high vacuum to maintain a negative-electron-affinity photocathode surface. The gun typically provided $\sim 7 \times 10^{10}$ electrons in the $2 \mathrm{~ns}$ light pulse, an instantaneous current of $\sim 6 \mathrm{~A}$, with quantum efficiencies of up to $\sim 10 \%$. A charge limit phenomenon [26] was observed with low quantum efficiencies at bunch sizes less than the expected space-charge limit of $\sim 10^{11}$ electrons. The polarization achieved from the photocathode was $\mathcal{P} \sim 28 \%$, measured prior to installation with a Mott polarimeter and a similar source [27]. This value is less than the theoretical maximum of $50 \%$ due to the $715 \mathrm{~nm}$ laser wavelength (see Fig. 2.6) and spin diffusion in the bulk GaAs cathode used. ${ }^{2}$

\footnotetext{
${ }^{1}$ A "strained-lattice" GaAs cathode can achieve $100 \%$ polarization theoretically; such a cathode is now in use at the SLC [24].

${ }^{2}$ Other "thin" $(\sim 0.2 \mu \mathrm{m})$ photocathodes of GaAs and AlGaAs produce higher polarization but are not as robust as the "thick" ( $635 \mu \mathrm{m})$ photocathode used.
} 


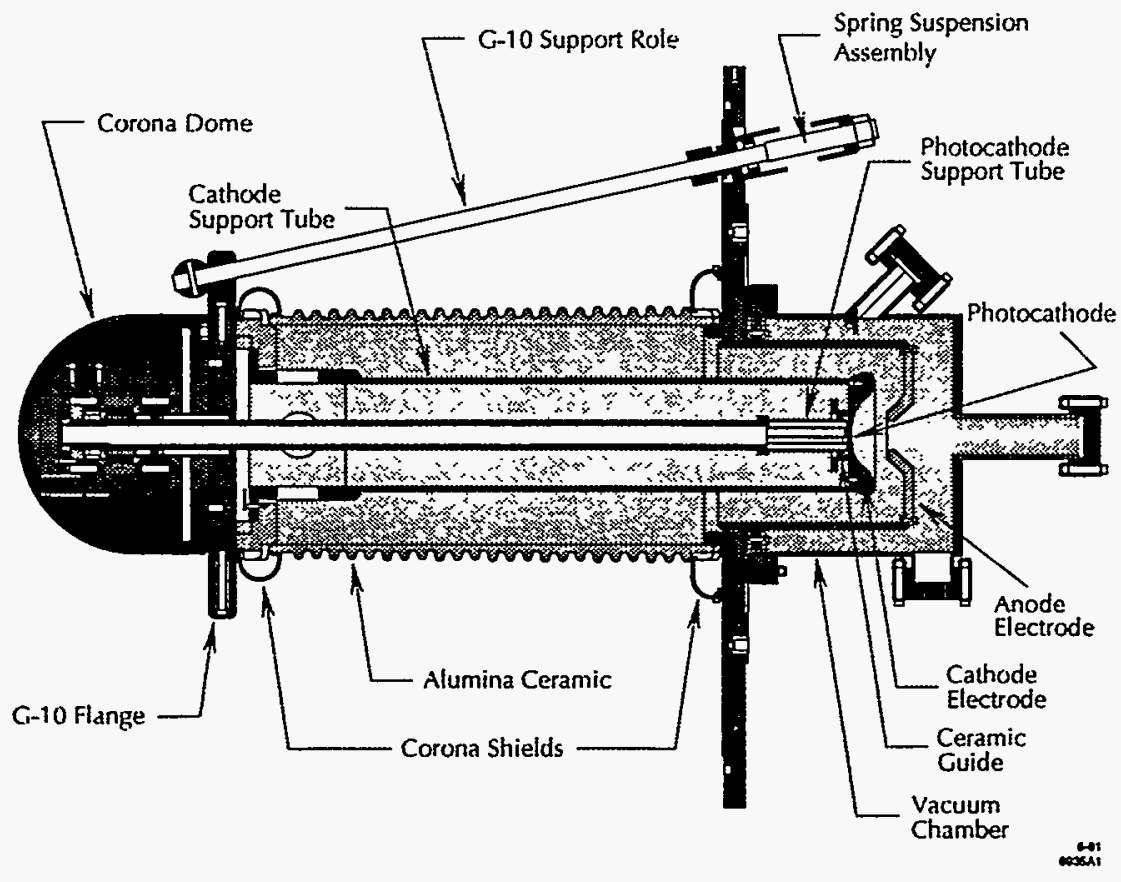

Figure 2.5: The SLC polarized electron gun.

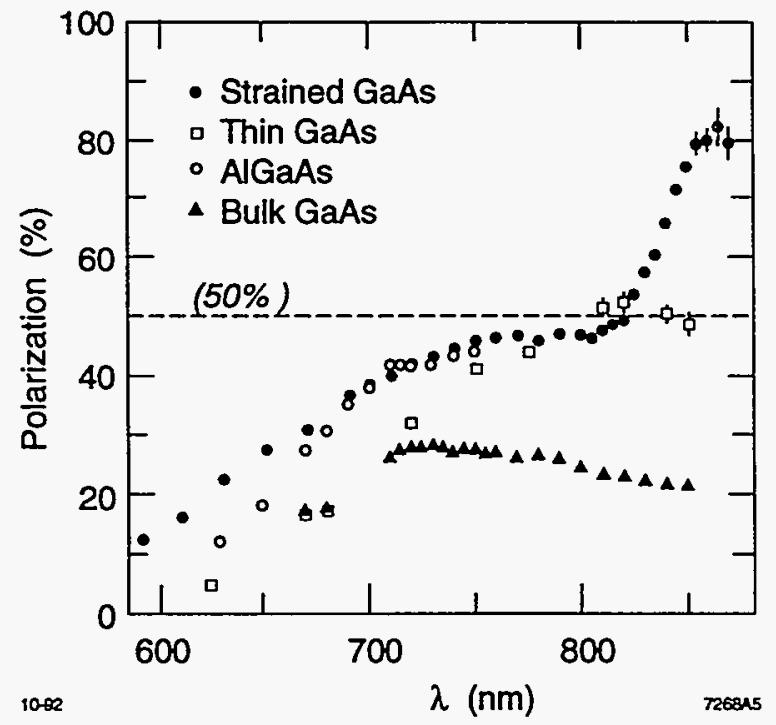

Figure 2.6: Polarization vs. wavelength for various GaAs photocathodes. This measurement was made with the bulk GaAs photocathode. 


\subsubsection{Spin rotation}

The phenomenon of spin precession in an electromagnetic field, first described by Thomas [28], confounds the delivery of longitudinally polarized beam to the interaction point (IP). The relationship between angular spin precession and precession of the momentum vector, for planar motion of the particle, is given by

$$
\theta_{\text {spin }}=\frac{\gamma(g-2)}{2} \theta_{e^{-}}
$$

where $\theta_{\text {spin }}$ is the angular spin precession with respect to the electron momentum vector, $\gamma$ is the Lorentz factor, $g$ is the gyromagnetic ratio of the electron, and $\theta_{e^{-}}$is the angular precession of the electron momentum vector. The energy dependence in this expression implies that any energy spread in the beam will lead to incoherent spin precession within the bunch, and the polarization will be lost if the beam is required to turn a large number of times in the SLC guide field. Such is the case in the north damping ring, where the electrons spend $\sim 8 \mathrm{~ms}$ and make tens of thousands of revolutions. In order to preserve the beam polarization, a system of solenoids is used to rotate the electron spins to vertical orientation upon entrance to the damping ring, so that there is no spin component in the horizontal bend plane, and then to reorient the spin vector in an arbitrary direction after leaving the ring. The north damping ring and spin-rotation solenoids are shown in Fig. 2.7.

The three spin rotators are identical 6.34 $\mathrm{T}-\mathrm{m}$ helium-cooled superconducting solenoids. This integrated field is chosen to rotate the spin of an electron at the nominal damping ring energy of $1.21 \mathrm{GeV}$ by $90^{\circ}$ about the solenoid axis. This energy also causes a spin precession of $90^{\circ}$ relative to the electron momentum precession in the plane of the damping ring for every $32.8^{\circ}$ horizontal bend of the electron. The first solenoid is located in the linac-to-ring (LTR) line, following a bend relative to the linac of $5 \times 32.8^{\circ}$. Hence, the electron spin at this point is horizontal and perpendicular to the beamline, and the LTR solenoid rotates the electron spins vertical. After the bunch is radiationdamped in the ring for one machine cycle, it is kicked out and passes through the ring-to-linac (RTL) solenoid. After exiting the RTL line, the beam traverses the linac solenoid, located $3 \times 32.8^{\circ}$ past the RTL spin rotator. Thus, depending on the field chosen for the RTL solenoid, the electron spin at the linac solenoid will have no transverse horizontal component and an arbitrary inclination. The linac solenoid can then choose any spin azimuth to create an arbitrary spin orientation down the linac.

In 1992 running, the damping rings were operated at $1.16 \mathrm{GeV}$, less than the design value, due to magnet saturation. This energy restriction had two effects. First, the spin at the LTR solenoid was 


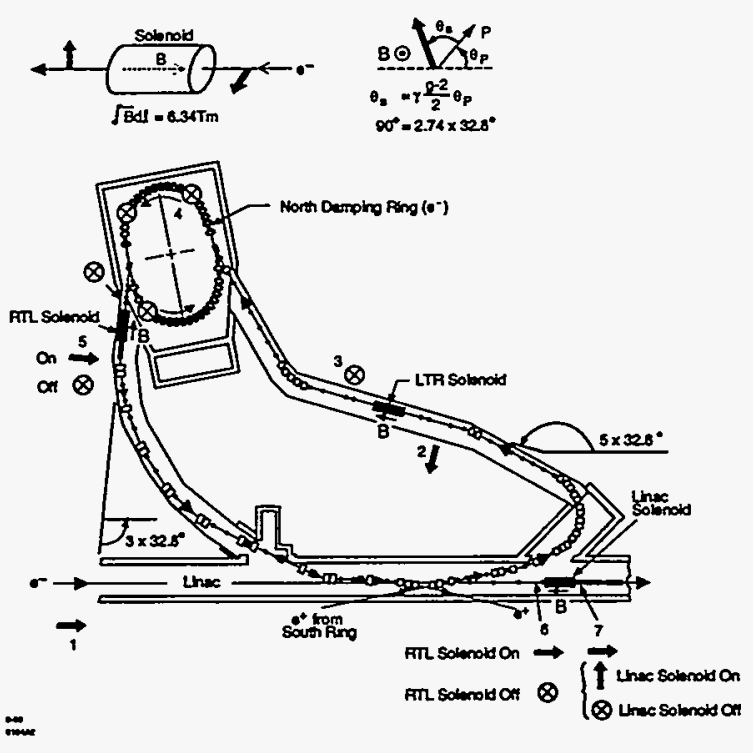

Figure 2.7: The north damping ring and spin-rotation solenoids. The arrow indicates the electron spin direction along the beam trajectory.

not completely transverse, as it precessed less than the required $5 \times 90^{\circ}$ from the linac to the LTR solenoid, leaving some longitudinal component, and so it could not be flipped exactly vertical. At the 1992 damping ring energy of $1.16 \mathrm{GeV}$, this horizontal spin component led to a depolarization $\left(\delta \mathcal{P}_{e} / \mathcal{P}_{e}\right)$ of $5 \%$. Second, completely longitudinal polarization could not be achieved down the linac, since the precession from the RTL solenoid to the linac solenoid was less than $3 \times 90^{\circ}$. This effect did not prove to be a limitation in the 1992 run. In general, freedom in choosing the spin orientation in the linac is necessary, as the horizontal and vertical rolls in the north arc lead to an unpredictable spin precession. In practice, the determination of the spin rotator currents was made empirically by measuring the longitudinal polarization at the IP for each of three orthogonal spin rotator setting combinations and using the resulting spin transfer matrix to set the solenoid currents to maximize the longitudinal IP polarization.

It was discovered early in the run that the north arc had another large effect on the transport of polarized beam. The betatron advance of one of the arc's 23 achromats is $1085^{\circ}$, while the spin precession in one of these achromats is $1080^{\circ}$. This coincidence causes a coupling of horizontal and longitudinal spin components to the vertical, and this coupling can build up through the arc to create a large vertical spin component at the IP [29]. The result is that the longitudinal polarization at the IP depends sensitively on the arc setup. An example is given in Fig. 2.8, where the longitudinal 
IP polarization is measured while the launch conditions at the entrance to the north arc are varied. The arcs proved difficult to maintain in an optimal configuration, and the longitudinal polarization

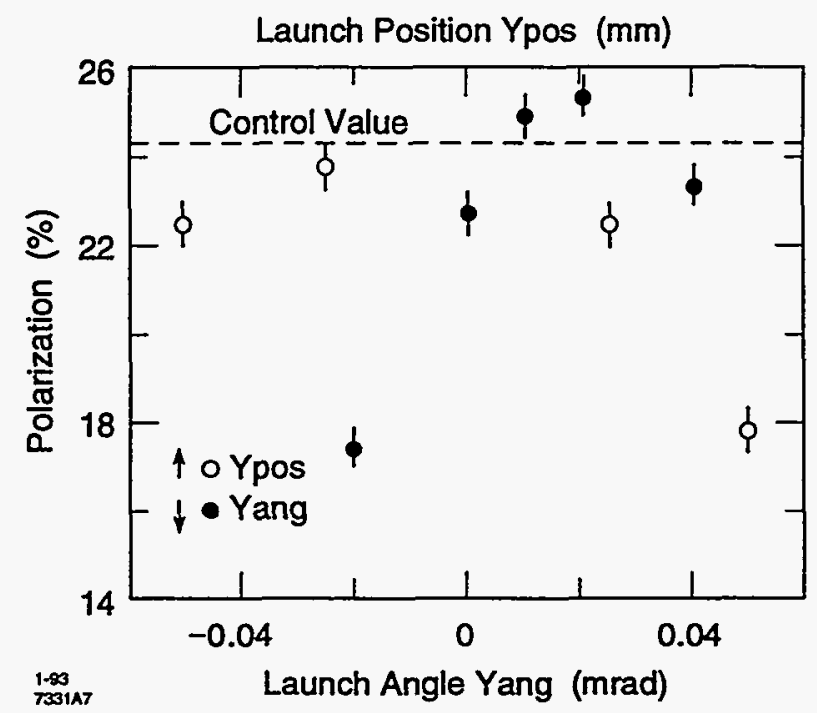

Figure 2.8: Longitudinal polarization at the IP as a function of north arc vertical launch angle (yang) and position (ypos).

at the IP accordingly took large $\left(\sigma_{\mathcal{P}} \sim 2 \%\right.$ ) excursions throughout the run.

There are a number of depolarizing effects in the process of transporting the beam that must be considered in comparing the measured polarization at the IP with the initial polarization from the gun. The biggest effect is the $5 \%$ relative polarization loss in the north damping ring; this depolarization was confirmed with large (5\%) errors by linac Møller polarimeter measurements on beam that had bypassed the north damping ring and on normally damped beam [27]. We expect another $\sim 5 \%$ depolarization in the north arc due to the inherent energy spread in the beam of $\sim 0.3 \%$. Other effects, including Sokolov-Ternov depolarization in the damping ring [30, 31], depolarization in the linac [32], and beam-beam depolarization at the IP [33], are all expected to be small $(<1 \%)$. Thus, we would expect a polarization of $28 \%$ from the gun to result in a longitudinal polarization at the IP of $0.95 \times 0.95 \times 0.28 \sim 25 \%$. The highest longitudinal polarization consistently measured at the IP was $\sim 26 \%$, while the all-run average was $22.4 \%$. This low average is attributed to the aforementioned sensitivity to arc setup. The conditions resulting in the highest longitudinal polarization at the IP proved too delicate to maintain for long periods of running. 


\subsubsection{Linac Møller polarimeter}

A polarimeter based on Møller scattering, located at the end of the linac in the PEP extraction line, is used for diagnostic measurements of the polarization before the beam enters the north arc. The device (Fig. 2.9) measures the polarization asymmetry in the cross section for polarized beam electrons on polarized electrons in an insertable magnetized iron target. The Møller-scattered

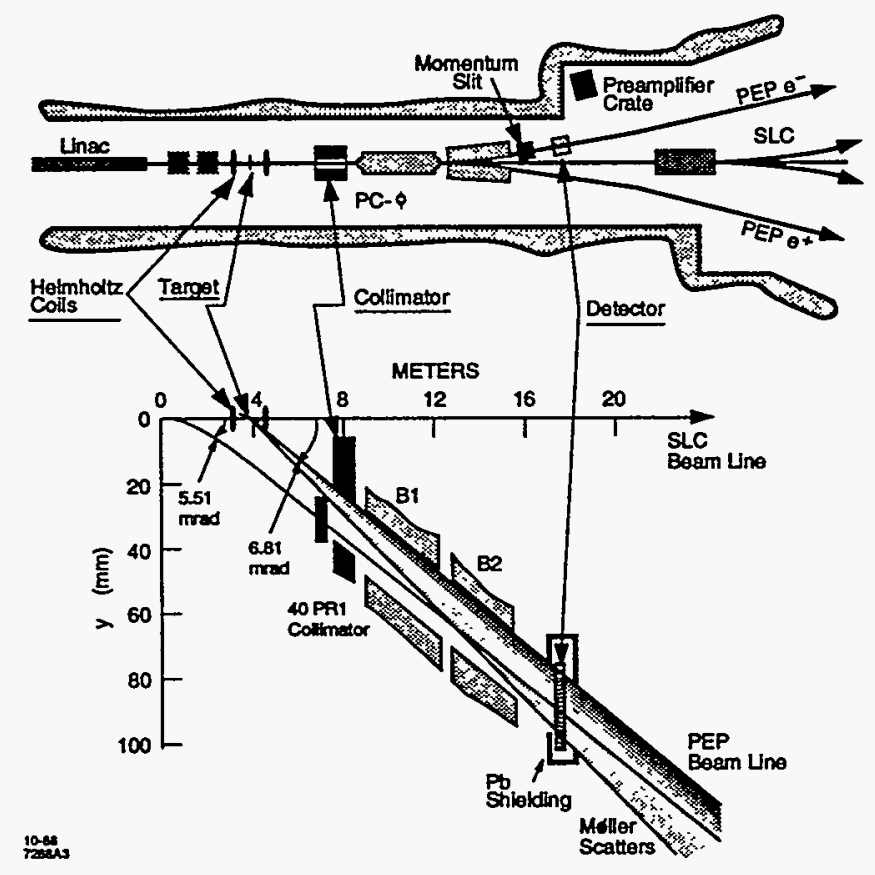

Figure 2.9: The linac Møller polarimeter.

electrons are momentum-resolved with the PEP extraction line magnets, and a collimator selects 10-15 electrons of $14-15 \mathrm{GeV}$ energy ( $110^{\circ}$ scattering angle in the cm frame) to enter a silicon strip detector. The beam and target polarizations are varied, and the longitudinal beam polarization is extracted:

$$
\mathcal{P}_{z}^{\text {beam }}=\frac{A^{\text {meas }}}{\mathcal{P}_{z}^{\text {trgt }} A_{z}}
$$

where $A^{\text {meas }}$ is the measured Møller asymmetry, $\mathcal{P}_{z}^{\text {trgt }}$ is the longitudinal target polarization, and $A_{z}$ is the longitudinal Møller asymmetry at the selected momentum (0.74 in this case). In principle, the polarimeter can also measure transverse beam polarization components, as there is a transverse Møller asymmetry, but the statistical precision of this measurement is much lower for a given run length. 
The dominant systematic errors in the Møller measurement are in the determination of the target polarization and the background. A variety of iron foil target thicknesses was available during the run; most often, the thin $(50.8 \mu \mathrm{m})$ foil was used. The measured polarization for this target was $8.49 \pm 0.26 \%$, contributing a $3.3 \%$ systematic error to the total of $\sim 5.5 \%$. The statistical error in a 30 -minute measurement was $\sim 2 \%$.

\subsubsection{Energy spectrometers}

After the electron beam collides with the positrons, it passes through the Compton polarimeter, which is discussed below, and then $120 \mathrm{~m}$ past the polarimeter reaches the south energy spectrometer [34]. The positrons are measured in a similar spectrometer on the north side. In the spectrometer (Fig. 2.10), the beam passes through a succession of dipole magnets. The first small bend sweeps

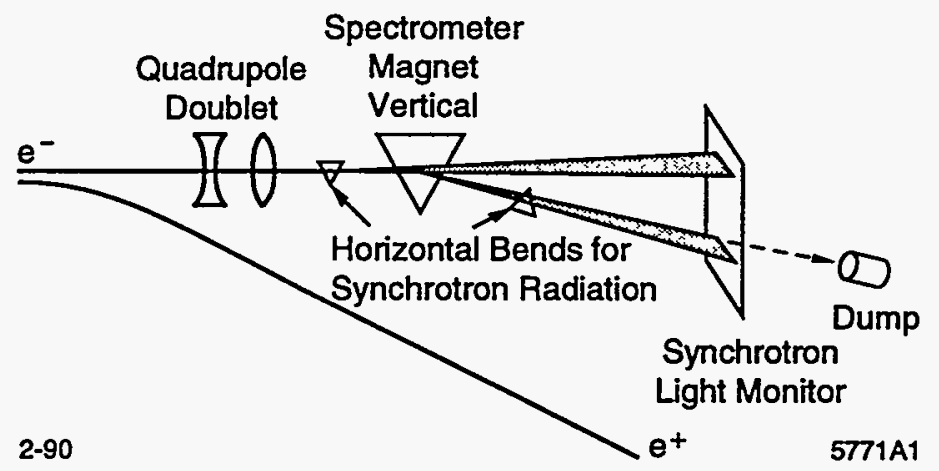

Figure 2.10: Schematic of the extraction-line energy spectrometer.

the beam horizontally by $3.1 \mathrm{mr}$, creating a swath of synchrotron radiation which is imaged onto a wire detector $15 \mathrm{~m}$ downstream. Then the precisely measured large vertical magnet bends the beam down by $\sim 18.3 \mathrm{mr}$ for the SLC beam energy of $\sim 45.6 \mathrm{GeV}$. Finally, the last small dipole sweeps the beam horizontally by $3.1 \mathrm{mr}$ in the other direction, creating another synchrotron radiation stripe on the wire-imaging detector. The distance between the stripes $(\sim 26 \mathrm{~cm})$, along with knowledge of the vertical bend strength and the distance to the center of the vertical magnet, then determines the beam momentum. In this way, the electron and positron beam energies are measured continually and $E_{c m}$ is known to $\pm 35 \mathrm{MeV}$. During the 1992 run, the average center-of-mass energy was $91.55 \pm 0.04 \mathrm{GeV}$, and the average electron energy was $45.68 \pm 0.03 \mathrm{GeV}$ [35]. 


\subsubsection{Extraction-line Møller polarimeter}

A second Møller polarimeter occupies the same location in the south extraction line as the south energy spectrometer. This polarimeter was intended to provide a check on the Compton polarimeter, as well as to measure transverse polarization components. The iron-foil and magnet target assembly is identical to that of the linac Møller polarimeter. The extraction-line device selects scattered beam electrons of half the beam energy, corresponding to $90^{\circ}$ scattering in the $\mathrm{cm}$ frame. This angle corresponds to the maximum longitudinal asymmetry of 0.78 . The detector is a sixteen-channel proportional-tube device with small-diameter tubes $(2 \mathrm{~mm})$ for high angle resolution.

The location of the south energy spectrometer bend magnets between the Møller target and detector caused ultimately insurmountable problems for the polarimeter. Off-energy beam electrons were bent in the spectrometer magnet fields and scattered from various beamline components, creating high backgrounds that overwhelmed the Møller detector. Thus, the device was never used to measure beam polarization. However, turning off the energy spectrometer magnets and repositioning the detector allowed the polarimeter to distinguish between left- and right-handed electron beam and hence provide a determination of absolute beam helicity [36].

\subsection{The Compton Polarimeter}

In order to extract $A_{L R}$ from the measured raw $Z$ asymmetry, the longitudinal beam polarization at the SLC IP must be known at the time each $Z$ is created. This measurement is the task of the Compton polarimeter, shown in Fig. 2.11. Circularly polarized optical photons collide with longitudinally polarized beam electrons at a Compton IP $33 \mathrm{~m}$ downstream of the SLC IP. The Compton cross section for doubly polarized electron-photon scattering has the energy and longitudinal polarization dependence

$$
\left(\frac{d \sigma}{d x}\right)_{\text {Comp }}=\left(\frac{d \sigma}{d x}\right)_{\text {unpol }}\left[1-\mathcal{P}^{\gamma} \mathcal{P}_{z}^{e} A_{z}^{e \gamma}(x)\right]
$$

where $x$ is the normalized scattered photon energy fraction, $\mathcal{P}^{\gamma}$ and $\mathcal{P}^{e}$ are the photon and electron polarizations, $A_{z}^{e \gamma}(x)$ is the Compton asymmetry function, and $\left(\frac{d \sigma}{d x}\right)_{\text {unpol }}$ is the unpolarized Compton cross section; these functions are shown in Fig. 3.2 in Chapter 3, where the Comptonscattering process is developed in detail. The scattered electrons continue forward and are swept by a dipole magnet into the Compton detectors, which analyze the polarization-dependent momentum spectrum of the electrons, from which the longitudinal beam polarization is determined. The Compton polarimeter continually operates in runs of a few minutes so that the slowly varying beam 


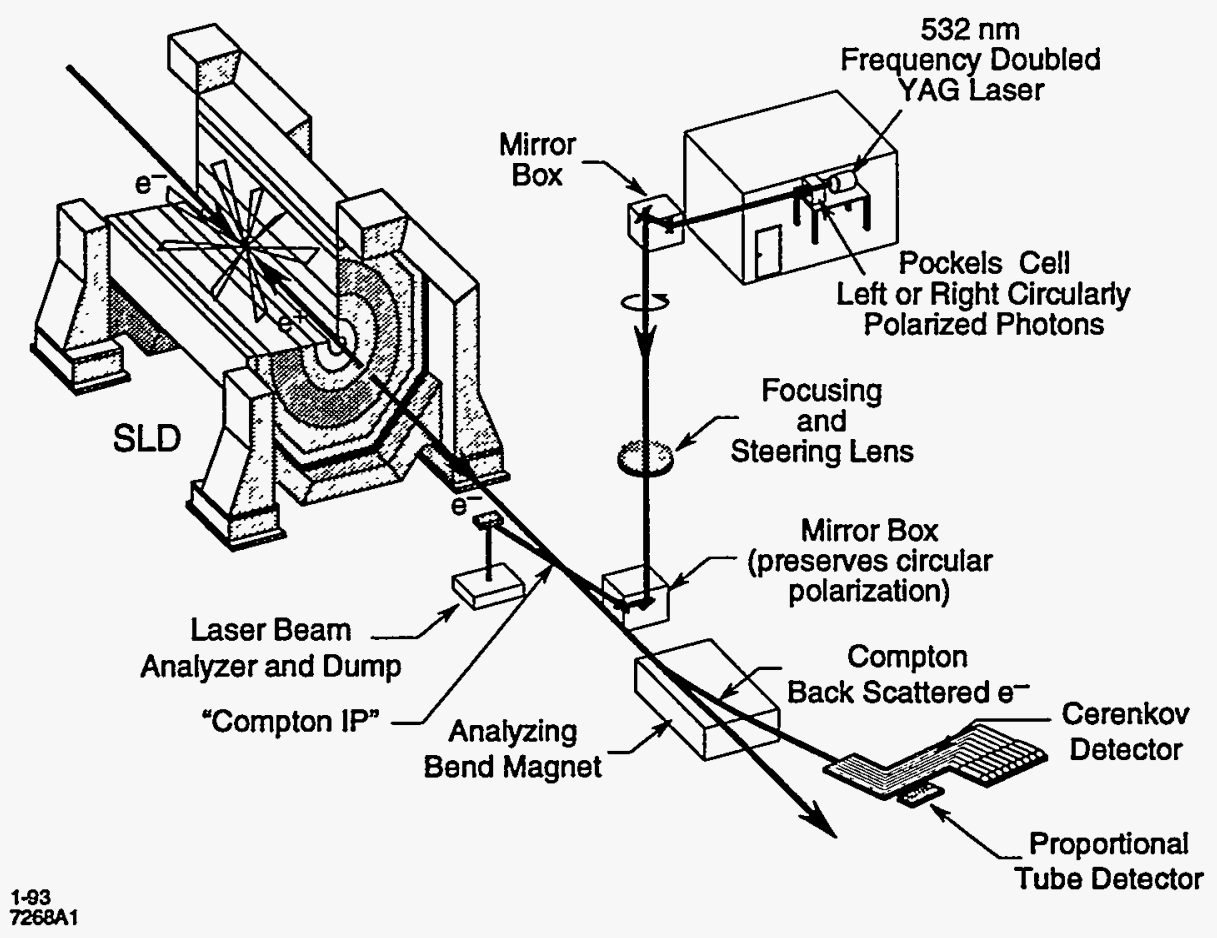

Figure 2.11: Schematic diagram of the Compton polarimeter.

polarization is known close in time to each $Z$ logged by the SLD. Compton polarimetry has the advantages over the Møller technique of high measured asymmetry (due to almost complete target polarization), large acceptance, no intrinsic background process, and smaller error in measuring the target polarization.

\subsubsection{Light source, transport, and monitoring}

The Compton laser light source provides high-intensity pulses of circularly polarized light which must then be transported with minimal loss of polarization to the Compton IP. In addition, the circular polarization of the light must be well measured and monitored throughout the run.

The Compton laser and associated source optics and polarimeter electronics reside in a trailer south of the Collider Experimental Hall. The laser bench, holding the laser and optics, is shown in Fig. 2.12. The laser is a Spectra Physics GCR-11 frequency-doubled Nd:YAG device producing $532 \mathrm{~nm}$ (green) light. The laser is run in Q-switched mode during polarimeter operation, giving a pulse width of $7 \mathrm{~ns}$ (FWHM) and a pulse energy of $45 \mathrm{~mJ}$. The laser is triggered every eleventh beam crossing using the timing pulse from an SLC base rate trigger (TRBR). This signal passes through a 


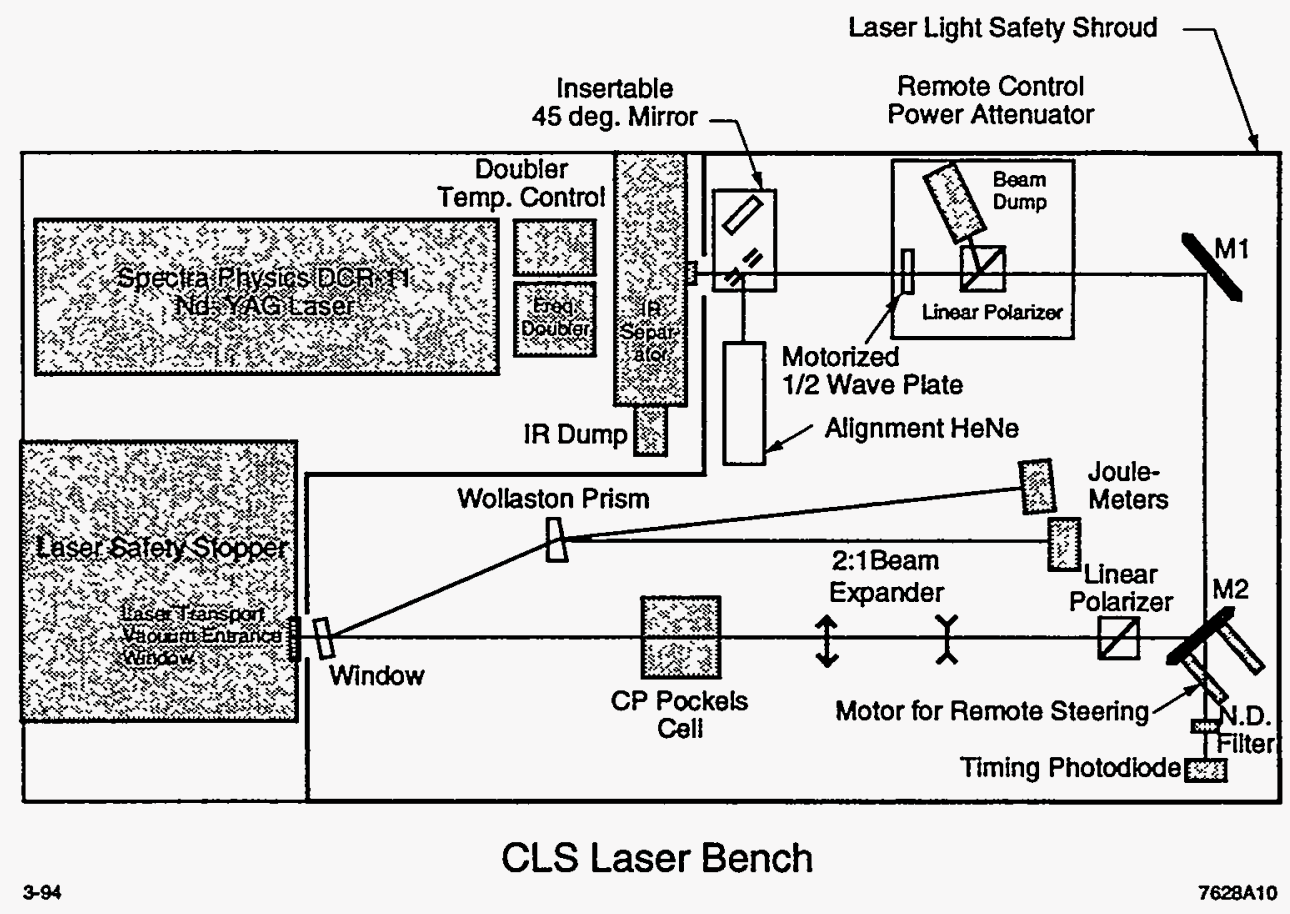

Figure 2.12: The Compton source laser bench layout.

CAMAC programmable delayed gate generator (LeCroy 2323), allowing the use of automated timing scans to synchronize the light and electron beams and thereby maximize the Compton signal. The light emerges from the laser linearly polarized. A remotely adjustable half-wave plate, in conjunction with a linear polarizer, attenuates the beam to the desired intensity to avoid damaging optical components on the bench and in the transfer line. A second linear polarizer follows two dielectric mirrors to clean up the beam. The beam is then expanded to $2 \mathrm{~cm}$ diameter to reduce the intensity on the optics and provide a collimated beam through the transport line to the Compton IP. At this point, the beam enters a Pockels cell to create circularly polarized light. This Pockels cell is operated in the same way as the polarized source Pockels cell in terms of both high-voltage control and pulse-by-pulse pseudo-random helicity selection $[23,37]$. After exiting the Pockels cell, the beam is partially (4\%) reflected from a canted glass window and through a Wollaston prism, which separates the two perpendicular light polarization components and directs them into two joulemeters. The ratio of intensities $R$ observed in the joulemeters provides a continual measurement of the circular polarization through the relation

$$
\mathcal{P}^{\gamma}=\frac{2 \sqrt{R}}{1+R}
$$


The light polarization measurement is discussed in the next chapter.

The light beam now enters the evacuated transport line, whose purpose is to preserve the circular polarization of the beam while delivering it to the Compton IP with the correct size and position. The beam first undergoes reflections in three sets of matched circular-polarization-preserving pairs of mirrors, and then passes through a lens of $5 \mathrm{~m}$ focal length to focus and steer the beam into collision with the electrons. The light beam was steered in this manner regularly throughout the run. After one more compensated reflection, the light enters the SLC vacuum at a $10 \mathrm{mr}$ angle with respect to the electrons. At the Compton IP the photon beam has an RMS size of $500 \mu \mathrm{m} v s$. $350 \mu \mathrm{m}$ for the electrons. After interacting with the electrons, the photons exit the SLC vacuum and, after an uncompensated reflection, enter an analysis box where a pair of joulemeters monitors the circular polarization using the same method as on the laser bench. This measurement is useful only to record changes in the circular polarization, not as an absolute determination, due to the last uncompensated reflection and a non-orthogonal Wollaston prism orientation with respect to the polarization ellipse. The light beam traverses a total of four vacuum windows, all of which were measured to have negligible birefringence both with the transport line at atmospheric pressure and evacuated.

The transport line vacuum can be broken to measure the light polarization at the Compton IP. This measurement was made before and after the run and will be discussed in detail in the next chapter. The photon polarization at both times was $93 \%$.

\subsection{2 Čerenkov detector}

The polarized electrons lose up to $28 \mathrm{GeV}$ with a characteristic polarization-dependent distribution in their interactions with the photon beam. This energy spectrum must be carefully measured in order to extract the electron beam polarization. A detector for this purpose should have excellent position resolution and good background suppression. A simple Čerenkov design was selected with these goals in mind.

\section{Analyzing magnets}

A layout of the Compton IP region is shown in Fig. 2.13. The electrons at the Compton IP have a beam divergence of $\sim 50 \mu$ r. The Compton process does not increase this divergence and so the scattered electrons remain with the unscattered beam until the set of analyzing magnets SB1 and B1 is reached. These magnets are the original bend dipoles for the incoming positron beam. The hard 


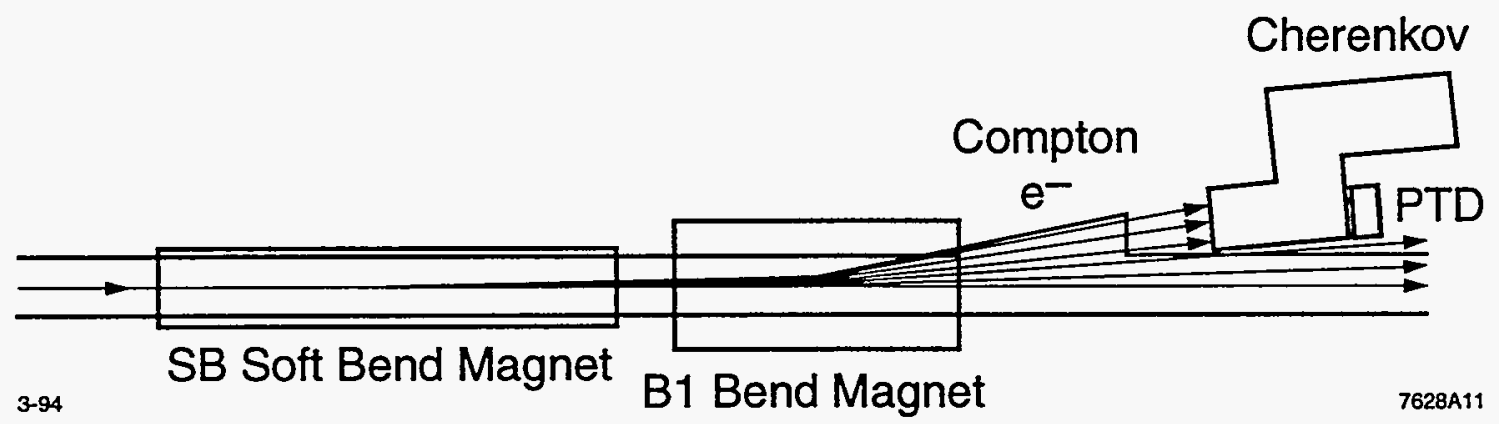

Figure 2.13: The Compton IP region. Electrons enter from the left.

bend $\mathrm{B} 1$ is designed to produce a deflection of $17.27 \mathrm{mr}$ for the on-energy beam, while the soft bend SB1 adds $1.00 \mathrm{mr}$ for this energy. The pair can be considered a single dipole with effective nominal bend strength of $833.2 \mathrm{MeV}$ transverse momentum kick and a unique effective center-of-bend point.

\section{Detector body}

About 3.5 meters past the effective bend point, the Compton-scattered electrons arrive at the Cerenkov detector. The detector, shown in Fig. 2.14, is a transversely segmented nine-channel device starting $\sim 4 \mathrm{~cm}$ from the beampipe, or $\sim 10 \mathrm{~cm}$ from the undeflected beamline extrapolated from the Compton IP.

The initial radiator section of the detector consists of channels $1 \mathrm{~cm}$ wide and $20 \mathrm{~cm}$ long separated by $250 \mu \mathrm{m}$-thick aluminum 6061 walls. All reflective surfaces throughout the detector interior are buffed along the channel axis and coated with $1000 \AA$ pure aluminum. The detector is canted relative to the beampipe and the walls in the front section are projective to point back to the effective bend point and hence to better define channel acceptances. Čerenkov radiation created in the space between the detector body and the start of each channel is blocked by thin aluminum tabs at the beginning of the channels. Polished, aluminum-coated stainless steel mirrors reflect Cerenkov photons created in the front section into the bend section, which moves the back end of the detector out of the path of the Compton electrons, and then into the final straight section. The channel height in all sections is $1.5 \mathrm{~cm}$, completely accepting the vertical electron spread of a few hundred microns; the width increases to $1.4 \mathrm{~cm}$ in the bend section and to $1.7 \mathrm{~cm}$ in the back section to reduce the number of photon reflections. The transmission efficiency through the detector for photons of $253.7 \mathrm{~nm}$ at the Čerenkov angle of $50 \mathrm{mr}$ has been measured to be $\sim 55 \%$. The interior of the detector is filled with the Čerenkov medium, cis and trans-2-butene at atmospheric temperature 


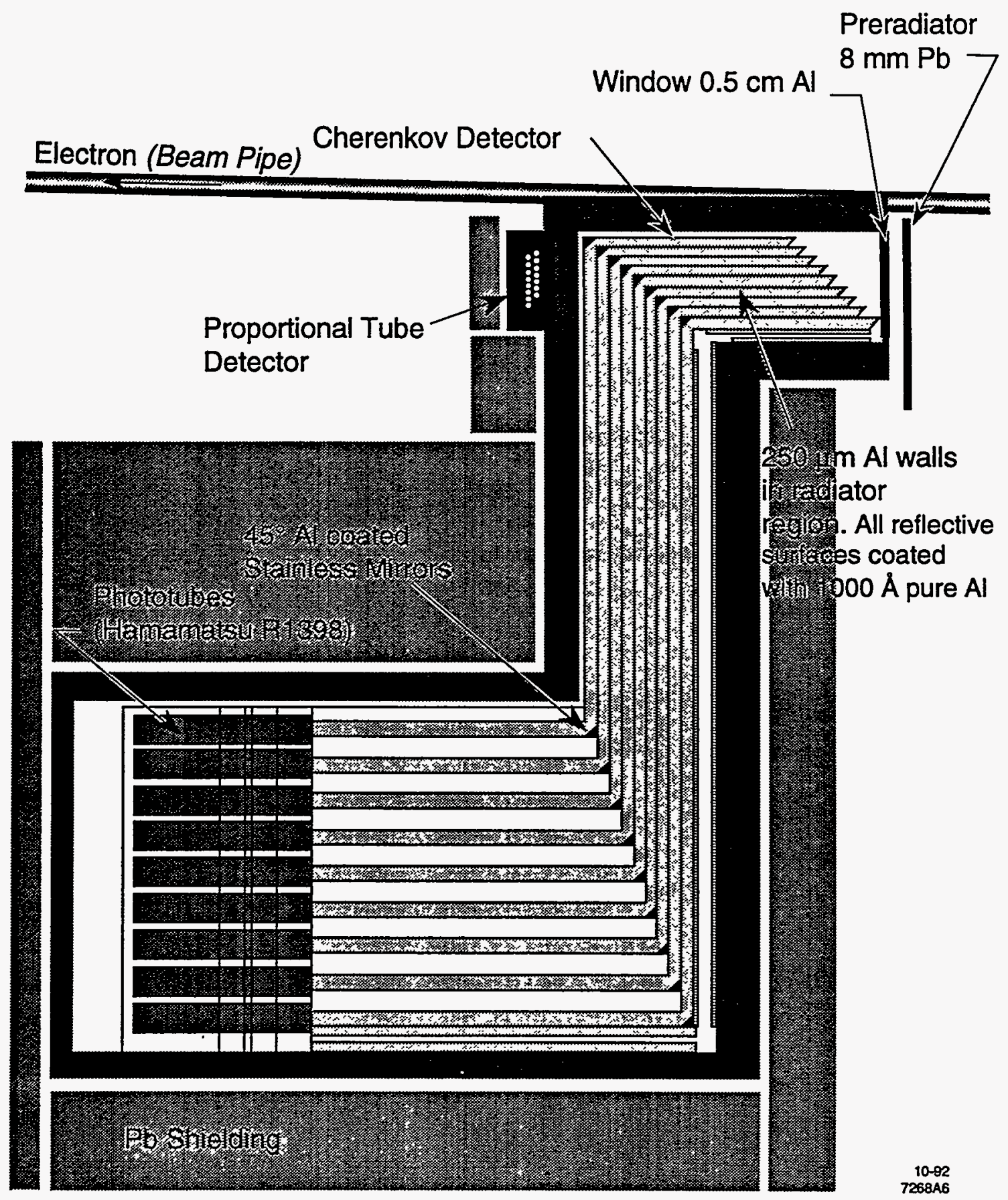

Figure 2.14: Overhead view of the Compton Cerenkov and proportional tube detectors. The electrons enter from the right. 
and pressure. This gas was chosen for its low scintillation and high ( $\sim 10 \mathrm{MeV})$ Čerenkov threshold. High-energy electrons produce $\sim 5$ Čerenkov photons per $\mathrm{cm}$ of travel through the gas. The vacuumtight detector body is constructed of aluminum 6061 with a $0.5 \mathrm{~cm}$-thick entrance window and gas and electronics ports at the back end. The entire detector past the front radiator section is shielded with several inches of lead on all surfaces. The Čerenkov detector, lead shielding, and proportional tube detector sit on a remotely movable table that can be translated horizontally to perform position scans. A precision linear potentiometer readback provides relative position information. In order to shield against collision-related backgrounds (apparently rescattered products of "beamstrahlung" radiation emitted during $e^{+} e^{-}$collision) and increase the signal-to-background ratio, it was necessary to include remotely insertable lead shields of thickness $2.54 \mathrm{~mm}$ and $5.08 \mathrm{~mm}$ immediately before the detector entrance. These lead shields replaced the original plates of thickness $8.5 \mathrm{~mm}$ and $16.9 \mathrm{~mm}$ early in the run when it was observed that $<10 \mathrm{~mm}$ of lead provided sufficient beamstrahlung shielding; however, the lead-in Compton edge scan described in the next chapter was performed using the original set of plates with $8.5 \mathrm{~mm}$ shielding.

\section{Phototubes/bases}

Due to the large detected Compton signal, linearity of the Čerenkov detector is a necessary characteristic. The measurement of the Compton asymmetry makes no requirement on knowledge of absolute gain; however, a nonlinearity in detector or electronics will bias the measured asymmetry. The linearity of the Čerenkov detector was an early concern: in the SLD engineering run in Fall 1991, the detector exhibited a severe drop in response as pulse height increased. This effect was soon discovered to be saturation in the Hamamatsu R292 phototubes used, occurring with as few as $\sim 10$ photons per pulse incident on the photocathode, in contrast to the several thousand per pulse expected in SLC running. A systematic program was launched to develop a tube/base combination that could linearly detect these light levels.

Space-charge saturation in the last stages of phototube multiplication is a well-known phenomenon. The relationship between the maximum current possible without saturation through a gain stage and the voltage across the stage is given by

$$
I_{\max }=k V_{\text {stage }}^{\frac{3}{2}}
$$

where $k$ is a proportionality constant determined by geometry. To avoid saturation, therefore, the voltage at the back of the tube must be high, while the current through the front end is kept low by 
decreasing the gain in the initial stages. The traditional method to achieve these goals is to use a tapered resistor chain that provides a progressively higher field in the later stages. The base design used in the Čerenkov detector (Fig. 2.15) is an improvement on this idea.

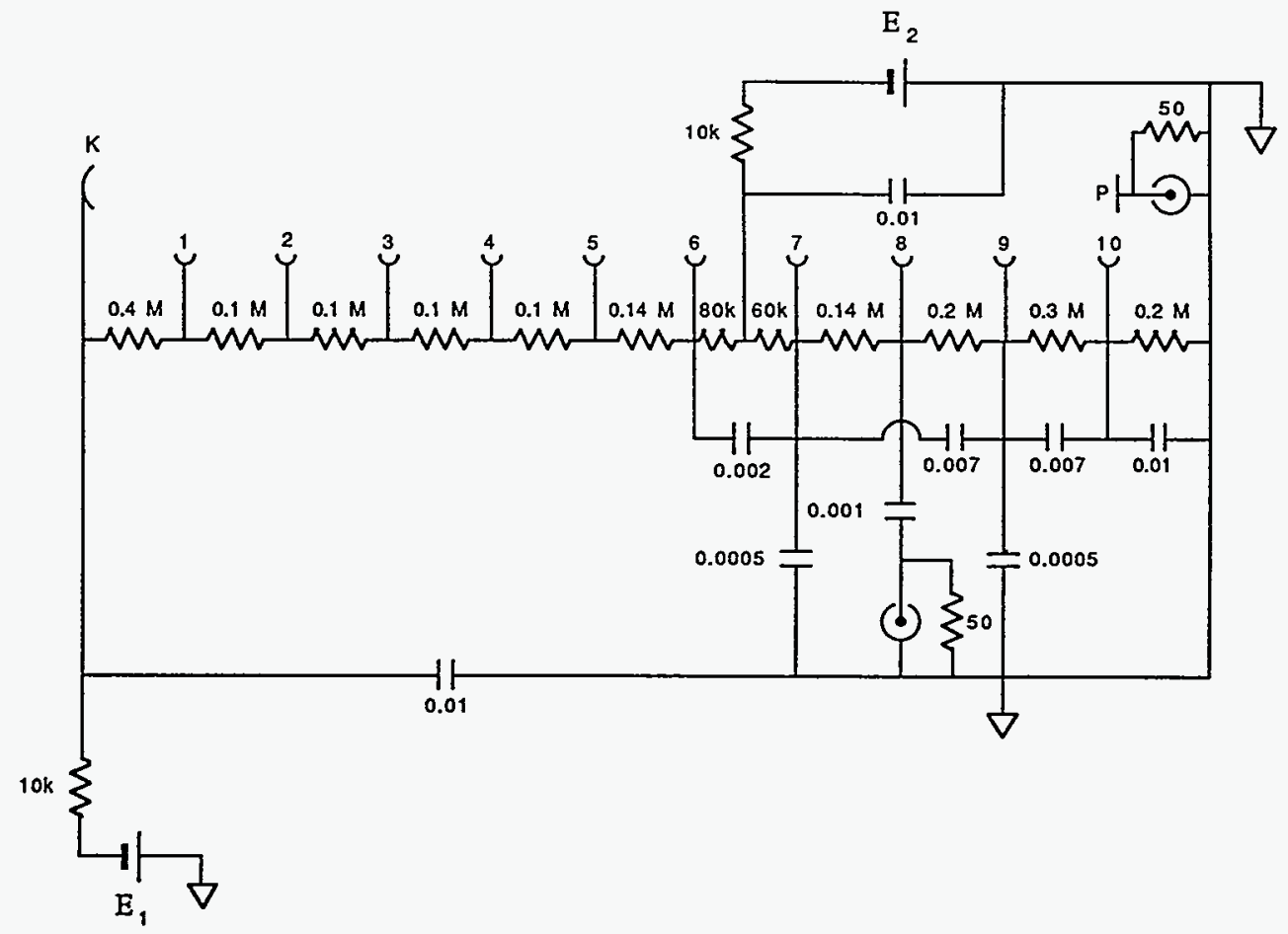

Figure 2.15: Schematic of Cerenkov detector phototube base. $E_{1}$ and $E_{2}$ are the two independent applied voltages. All capacitances are in microfarads.

Central to the base design is the two-voltage scheme, whereby a second independent potential is applied to the back end of the base to allow separate adjustment of the gain in the tube, controlled with $\epsilon_{1}$, and the space-charge clearing voltage, controlled with $\epsilon_{2}$. The resistor chain is a standard tapered design, with small fields in the first stages and a gradual increase through the tube. The resistance progression is chosen so that the voltage increases by less than $50 \%$ in consecutive stages to preserve the designed electric field map in the phototube. The back-end voltage $\epsilon_{2}$ enters in the middle of stage 7 to allow a greater range of $\epsilon_{1} / \epsilon_{2}$ voltage combinations while obeying this $50 \%$ voltage-step rule. Capacitors across the final stages maintain the interdynode potentials even with large instantaneous currents. The RC time is designed to be over ten times shorter than the SLC period of $8 \mathrm{~ms}$, and the circuit was tested at ten times the SLC rate with no observable effect on 
the response. A second output is available for each base at dynode 8; however, the dynode signal proved to be too small to be usable.

The phototubes used in conjunction with the two-voltage base are the Hamamatsu R1398, with a borosilicate glass window admitting photons from 300 to $650 \mathrm{~nm}$, and the otherwise identical Hamamatsu R1668, with a synthetic silica window for response from 160 to $650 \mathrm{~nm}$. These tubes are $11 / 8^{\prime \prime}$, eleven-stage designs with a linear-focused dynode chain for high linearity. The rise time of the tubes is $2.2 \mathrm{~ns}$; since the photon pulse arrives at the photocathode essentially instantaneously in comparison, the rise time defines the leading edge of the output pulse, and the width is $\sim 2 \mathrm{~ns}$. The tubes vary widely in gain, by up to a factor of $\sim 5$, but again absolute gain has no effect on the measured asymmetry. Extensive testing of the tube/base combination was performed in 1991 in order to characterize the linearity of the system as a function of incident light level and $\epsilon_{1} / \epsilon_{2}$ voltage combination. The resistance taper is such that in order to maintain less than a $50 \%$ voltage gain in consecutive stages, $\epsilon_{1}$ must be at least $50 \%$ greater than $\epsilon_{2}$. Since more incident light can be measured linearly the smaller the gain of the tube and the larger the field in the back, it is desirable to set $\epsilon_{1} / \epsilon_{2}$ as little above 1.5 as possible. Figs. 2.16 and 2.17, based on data from 1991 lab tests, support the hypothesis that saturation is due to space-charge in the later stages of the phototube. The point at which nonlinearity occurs in the output pulse is independent of $\epsilon_{1}$ but strongly dependent on $\epsilon_{2}$.

Prior to installation, the bases were potted under vacuum in Stycast to prevent damage due to sparking. Mu-metal shields were placed around the phototubes. An internal LED pulser system was installed to provide a diagnostic check for signal in each phototube. The limited number of high-voltage ports on the detector body (it was designed prior to the two-voltage base) necessitated the use of a single common $\epsilon_{2}$ voltage for all tubes, while $\epsilon_{1}$ could be set independently channelto-channel. Channels 1 and 9 had only $\epsilon_{1}$ connected. Channel voltages changed a number of times during the run due to unstable beam conditions; most of the running was done with $\epsilon_{2}=600 \mathrm{~V}$ and $\epsilon_{1} \sim 1000 \mathrm{~V}$. This voltage combination gives a gain of $\sim 20,000$.

\subsubsection{Proportional tube detector}

The second Compton detector, the Compton proportional tube detector (PTD), samples the scattered electron spectrum immediately after the Čerenkov detector. The position of the PTD is shown in Fig. 2.14; construction details are given in Fig. 2.18.

The PTD is, in essence, "an instrumented lead brick" [38]. The instrumention consists of sixteen 


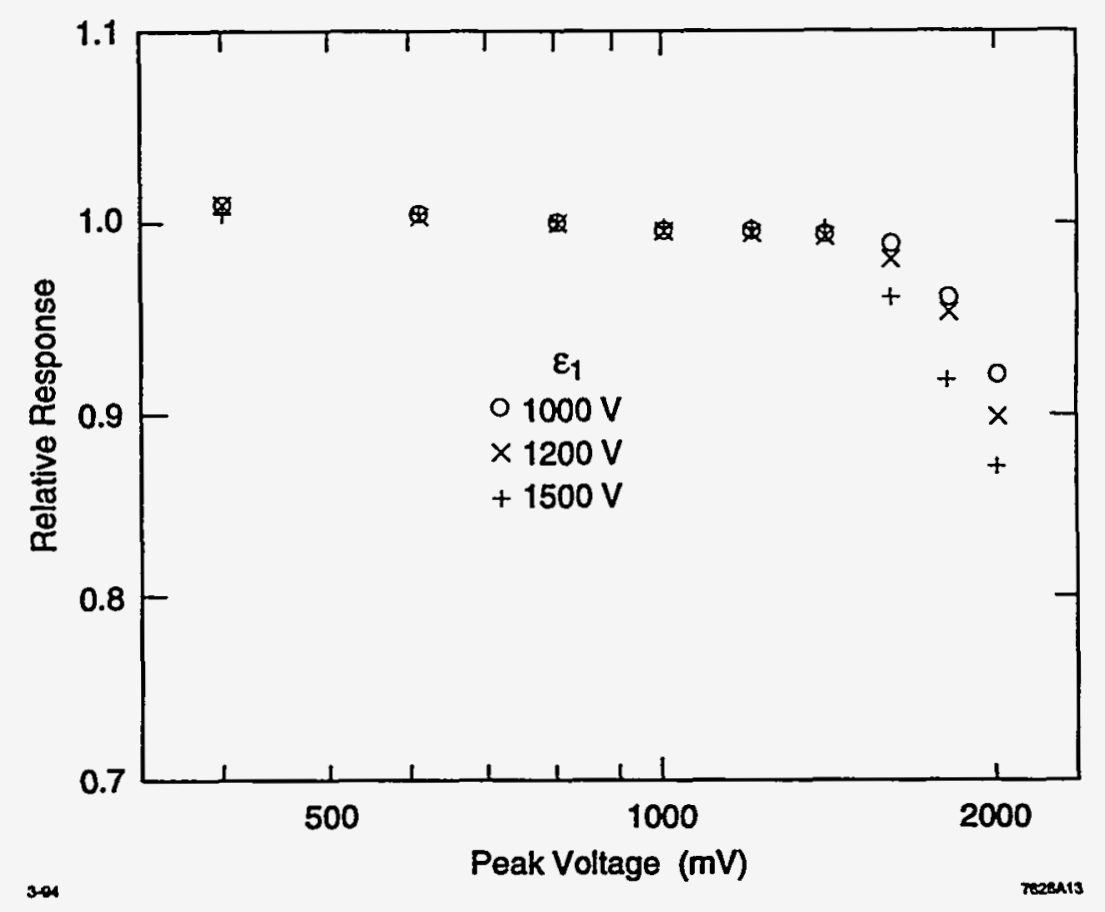

Figure 2.16: The normalized linearity of a typical phototube/base combination for $\epsilon_{2}=600 \mathrm{~V}$ and three different $\epsilon_{1}$ values. The dropoff is independent of $\epsilon_{1}$.

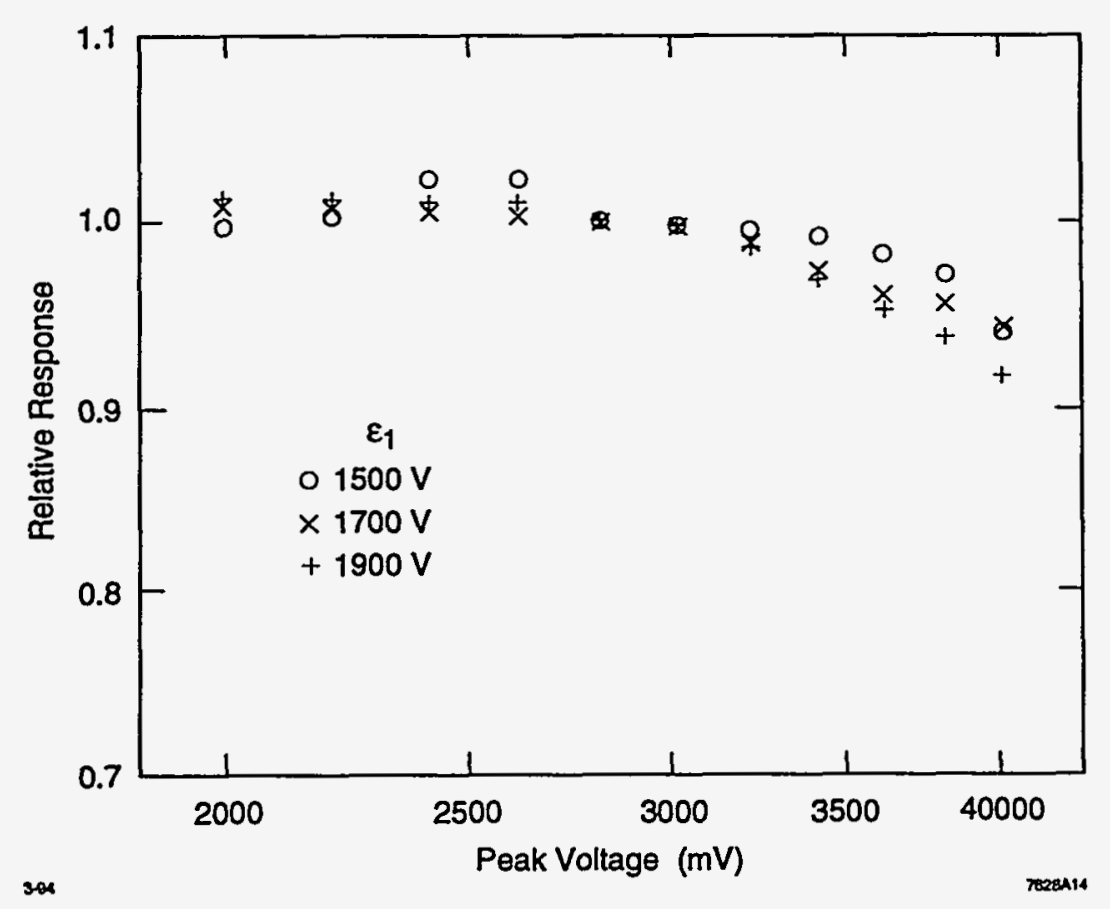

Figure 2.17: Linearity of same phototube/base with $\epsilon_{2}=1000 \mathrm{~V}$ for different $\epsilon_{1}$ values. Again the dropoff is independent of $\epsilon_{1}$ but occurs at a much higher output than with $\epsilon_{2}=600 \mathrm{~V}$. 


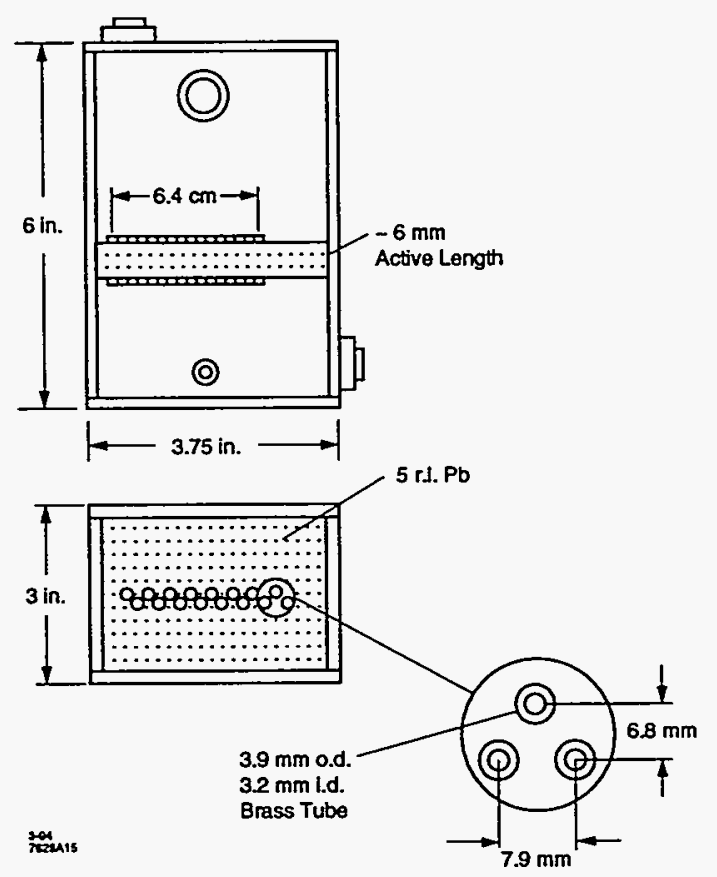

Figure 2.18: The Compton proportional tube detector. The top picture is the back of the detector; the bottom is a view from above with electrons entering from the top.

$26 \mathrm{~mm}$-long brass tubes of inner diameter $3.23 \mathrm{~mm}$ each surrounding a $20 \mu \mathrm{m}$-thick, $10 \mathrm{~mm}$-long field/charge-collection wire. The wire has an active length (flat gain region) of $6 \mathrm{~mm}$. This length was made as short as possible to reduce backgrounds. The tubes are staggered in the longitudinal direction by $6.8 \mathrm{~mm}$ and have an effective transverse spacing of $4.0 \mathrm{~mm}$. The active area of the tubes is embedded in a horizontal lead plate; the electrons shower through five radiation lengths before reaching the tubes. The PTD uses HRS gas, composed of $89 \% \mathrm{Ar}, 10 \% \mathrm{CO}_{2}$, and $1 \% \mathrm{CH}_{4}$, at atmospheric pressure and temperature, bubbled through water to avoid breakdown. The majority of 1992 running was done with $750 \mathrm{~V}$ on the wires, giving a gain of $\sim 60$.

It was discovered during running that the Compton background was systematically mismeasured in the PTD due to slow charge collection on the exposed endplug feedthroughs. For this reason, the detector was not used in the analysis to measure Compton scattering directly, but was used as a calibration point in studies of the Čerenkov detector linearity. The tubes have since been potted and the problem appears to have been solved for the 1993 run. 


\subsubsection{Electronics and data acquisition}

The Compton polarimeter relies on an assortment of electronic signals and devices for timing, control, measurement, and auxiliary information, both within the polarimeter and in conjunction with the SLC/SLD. In addition, a standalone data acquisition system accumulates and processes the data from each polarimeter run and sends summed results and status and machine information to the SLD, where it is written to tape.

\section{Compton electronics}

The Compton electronics system is based on three serial CAMAC crates, two in the laser shack and a third on the mezzanine of the collider hall linked to the first two by fiber optic cable.

The Compton electronics chain begins with the laser trigger. A dedicated SLC TRBR provides a trigger for the laser flashlamp and Pockels cell at $10.9 \mathrm{~Hz}$ (every eleventh beam crossing), while a second dedicated TRBR, passing through a CAMAC programmable delay unit (LeCroy 2323), triggers the laser Q-switch. An automated timing scan based on this unit was used approximately hourly during the run to synchronize the laser pulse with the electrons. A third TRBR, through separate delay gates, controls the timing of the ADCs reading the four laser-bench and analysis-box joulemeters, the nine Čerenkov channel signals and backgrounds, and the 16 PTD channel signals and backgrounds. The ADCs used are the LeCroy $2249 \mathrm{~W}$ (charge-sensing) for the Cerenkov detector and the joulemeters, and the 2259B (peak-sensing) for the PTD. The linearity curves of both ADC types were investigated, with negligible nonlinearity for the $2249 \mathrm{~W}$, but were not used to correct the response of the detectors. In addition, this third TRBR controls the timing of a delay gate for a 2249A $A D C$ receiving pulse-by-pulse north and south beamstrahlung monitor (BSM) data. Beam toroid data (electron and positron currents, north and south) is also read every pulse by a 2259B ADC. Digital information on the source electron helicity is sent to the polarimeter over two redundant lines. The Klystron Veto Module (KVM) line, running the length of the SLC and connected by KVMs, carries the helicity data in two bits parasitically along with other SLC information. A more reliable dedicated cable carries the source helicity bits directly to the Compton data acquisition. The polarimeter Pockels cell status bits are recorded in a bit register. Digital beam dumper information is also stored for data veto purposes. An Isolated Digital Input Module (IDIM) is used to read the status of the two Čerenkov detector lead shielding plates. Analog signals providing laser status information and Čerenkov and PTD table position, via a linear potentiometer, are monitored with a Smart Analog Module (SAM). Finally, several control signals are used in polarimeter operation. An 
Isolated Digital Output Module (IDOM) sends commands to operate the laser remotely via the SLD VAX. An IDOM is also used to insert or remove the Cerenkov detector lead plates via computer, to move the detector table, and to move the laser beam lens in both orthogonal directions to steer the beam and maximize the Compton signal, an adjustment that was made regularly throughout the run with an automated computer scan.

\section{Compton data acquisition}

Data for the Compton polarimeter was acquired with a standalone $\mu \mathrm{VAX}$, the M2ELN, communicating with the three Compton CAMAC crates on the polarimeter side through a Kinetic Systems serial driver and with the SLD VAX for integration into the SLD data structure. Every machine cycle $(120 \mathrm{~Hz})$, the last Compton trigger in the cycle sets a CAMAC LAM which causes the M2ELN to place into a ring buffer the 52 words of data produced by the polarimeter. For each combination of source helicity bit (L, R, Error) and Compton helicity bit (L, R, Off, Error), M2ELN writes the Cerenkov and PTD raw channel data into two arrays, one a sum over the run and the second a sum of squares over the run for error calculation. The joulemeter data is separated only on the basis of the laser state; the BSM and beam toroid data is separated only by electron state. This data also is written to summed and summed squares arrays. Data is not written if a data veto is in effect. These vetoes are: high noise in Čerenkov channel 9; electron dumper in; positron dumper in; low electron current; low positron current. At the end of a polarimeter run, typically 20,000 beam crossings, these data histograms are sent to the POLSERVER process on the SLD VAX, along with the ring buffer for the last 150 beam crossings. POLSERVER then reads the polarimeter SAM data and Pockels cell and Cerenkov lead status bits. This resulting set of histograms and raw data is copied on the SLD VAX into a data bank with a time stamp for association with SLD Zs .

\subsection{The SLD Detector}

The SLD (SLC Large Detector) was designed to be the main detector for SLC $Z$ physics. The SLD design was set forth in 1984 [39] and the detector was completed in 1991, replacing the Mark II detector at the SLC IP. The SLD is typical of "modern" detector designs: it includes a silicon vertex

tracker, a ring imaging Čerenkov detector, and compact electromagnetic and hadronic calorimetry inside the solenoidal magnetic field, in addition to the standard central and endcap drift chambers, luminosity monitoring, and external muon detection. The data is acquired almost exclusively through fastbus, and some preprocessing is done with local electronics mounted on the detector. A 
quadrant of the detector is shown in Fig. 2.19.

\subsubsection{Vertex detector}

The SLD vertex detector [40] is designed to distinguish vertices due to secondary decays of heavy quarks and $\tau$ leptons from the vertex of the primary $Z$ decay. The vertex detector, shown in Fig. 2.20, is composed of 480 silicon charge-coupled devices (CCDs) with a total of 120 Mpixels, each $22 \times 22 \mu \mathrm{m}$ pixel providing an independent measurement of track position. The $9 \times 13 \mathrm{~mm}$ CCDs are laid out in rows of eight along 60 "ladders", which are arranged in four concentric cylinders around the beampipe. The four barrels are situated at radii from $29.5 \mathrm{~mm}$ to $41.5 \mathrm{~mm}$ around the $25.5 \mathrm{~mm}$ SLC beampipe. The total thickness of the detector, including the beampipe, is $5.82 \times 10^{-2}$ radiation lengths. The detector is cooled to $-80^{\circ} \mathrm{C}$ with nitrogen gas to suppress dark current and loss of CCD charge-transfer efficiency from radiation damage. The detector achieves a typical tracking precision of $5 \mu \mathrm{m}$ in each space coordinate. The readout time for a vertex event is $160 \mathrm{~ms}$, or 19 beam crossings at $120 \mathrm{~Hz}$; thus every recorded event has superimposed on it the background from 19 beam crossings. The small pixel size and $20 \mu \mathrm{m}$ active silicon thickness, however, reduce the observed background below that for an equivalent-size microstrip detector.

\subsubsection{Drift chambers}

The SLD drift chamber system is composed of five parts: a central drift chamber (CDC) around the beampipe and two pairs of endcap drift chambers (EDCs) at different longitudinal positions.

\section{Central drift chamber}

The central drift chamber is a $2 \mathrm{~m}$-long cylinder of inner radius $20 \mathrm{~cm}$ and outer radius $1 \mathrm{~m}$. It is composed of $\sim 50 \mathrm{~mm}$-wide cells (Fig. 2.21) forming ten concentric superlayers (Fig. 2.22). Each cell consists of field-shaping wires, guard wires, and eight anode sense wires. The longest drift distance in any cell is $30 \mathrm{~mm}$. Drift distances can be determined to $\sim 100 \mu \mathrm{m}$, defining the transverse position resolution. The sense wires are read out on both sides of the detector, and charge division is used to determine the longitudinal hit position to $\sim 15 \mathrm{~mm}$. To resolve the leftright position ambiguity, six of the superlayers are $\pm 50 \mathrm{mr}$ stereo layers; the remaining four are axial layers. The $\mathrm{CDC}$ uses a $\mathrm{CO}_{2}$ - $\mathrm{Ar}$ gas with $\mathrm{H}_{2} \mathrm{O}$ to reduce carbon deposition on the sense wires and isobutane to increase gain and allow lower-voltage operation. This gas mixture has a slow drift velocity $(10 \mu \mathrm{m} / \mathrm{ns})$ and low diffusion for high time resolution. The analog sense-wire signals are 


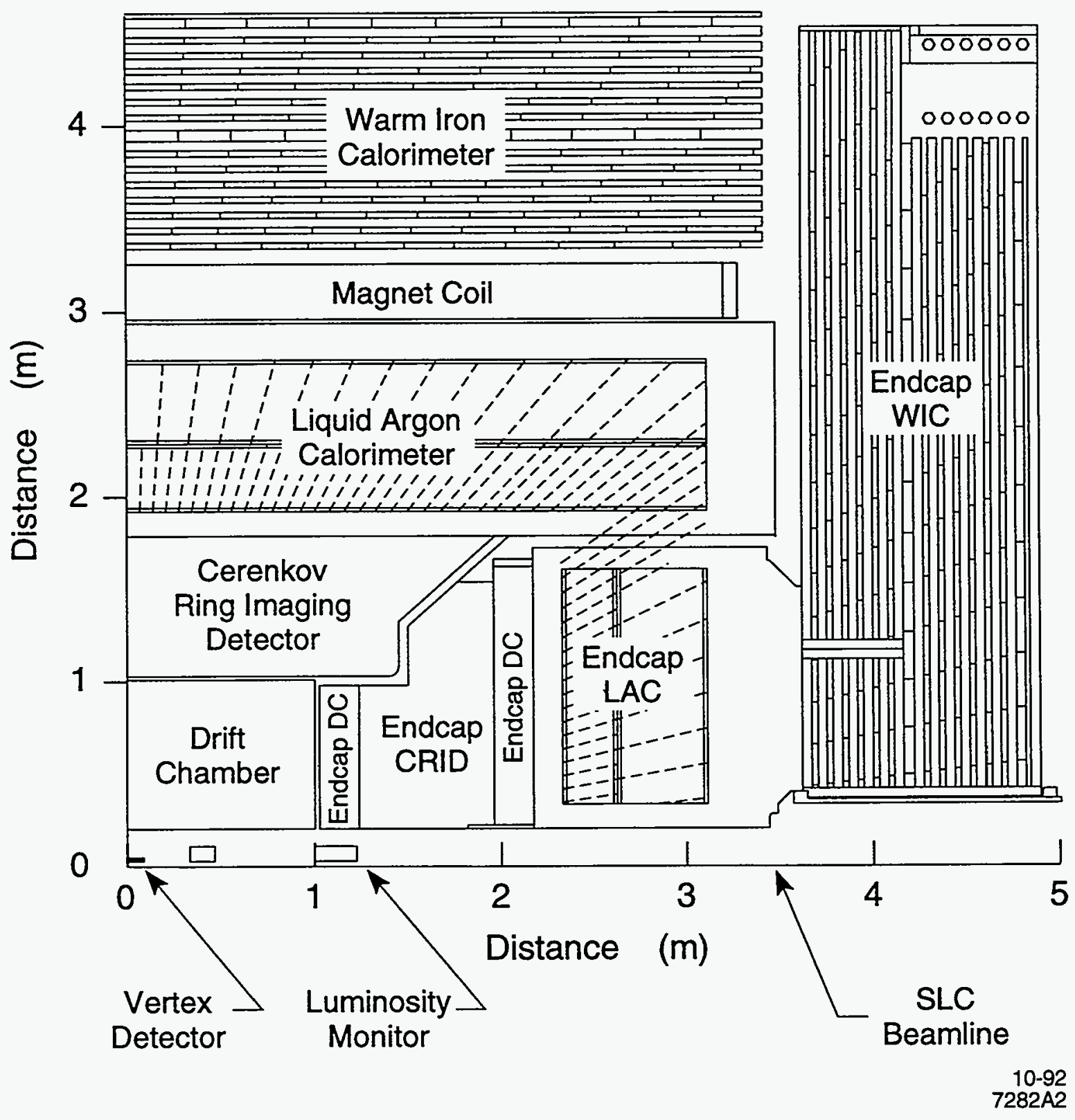

Figure 2.19: Quadrant schematic view of the SLD detector. 


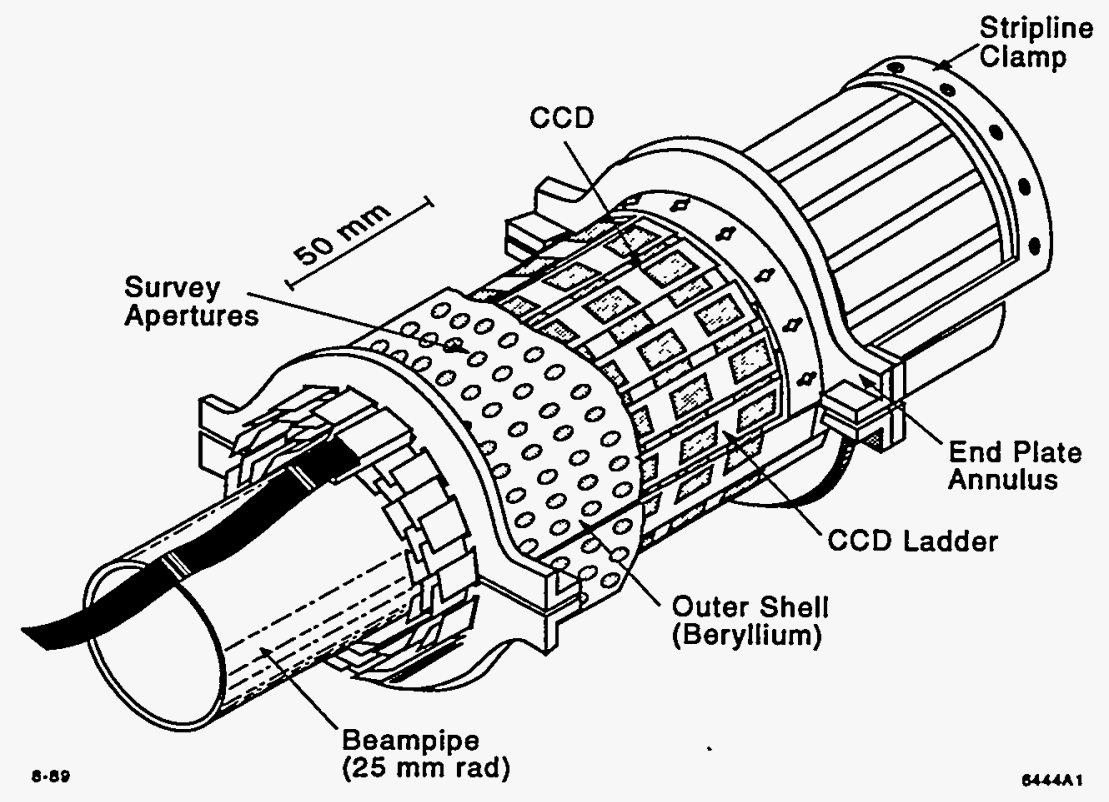

Figure 2.20: The SLD vertex detector.

read with preamplifiers mounted directly on the chamber. This data is then digitized locally and sent via optical fiber to the fastbus system.

\section{Endcap drift chambers}

Tracks with polar angles of less than $30^{\circ}$ are not well measured in the central drift chamber, as these tracks pass through only a small number of layers. The EDCs cover the region between $12^{\circ}$ and $40^{\circ}$ in polar angle. The two pairs of EDCs reside at $z$ positions of $1.12 \mathrm{~m}$ and $2.06 \mathrm{~m}$ from the IP. Both sets have inner radii of $0.2 \mathrm{~m}$, while the outer radii are $0.97 \mathrm{~m}$ and $1.65 \mathrm{~m}$ for the inner and outer EDCs respectively, subtending approximately equal solid angles. Each EDC is composed of three superlayers rotated $120^{\circ}$ with respect to each other. The inner- and outer-chamber superlayers respectively comprise 22 cells and 34 cells each, with six sense wires per cell in both chambers. The maximum drift distance in an EDC cell is $50 \mathrm{~mm}$, and the average resolution over a cell is $\sim 140 \mu \mathrm{m}$. The left-right position ambiguity in the EDC is resolved by staggering the sense wires $150 \mu \mathrm{m}$ from the center of a cell and choosing the reconstructed track with the better $\chi^{2}$. 


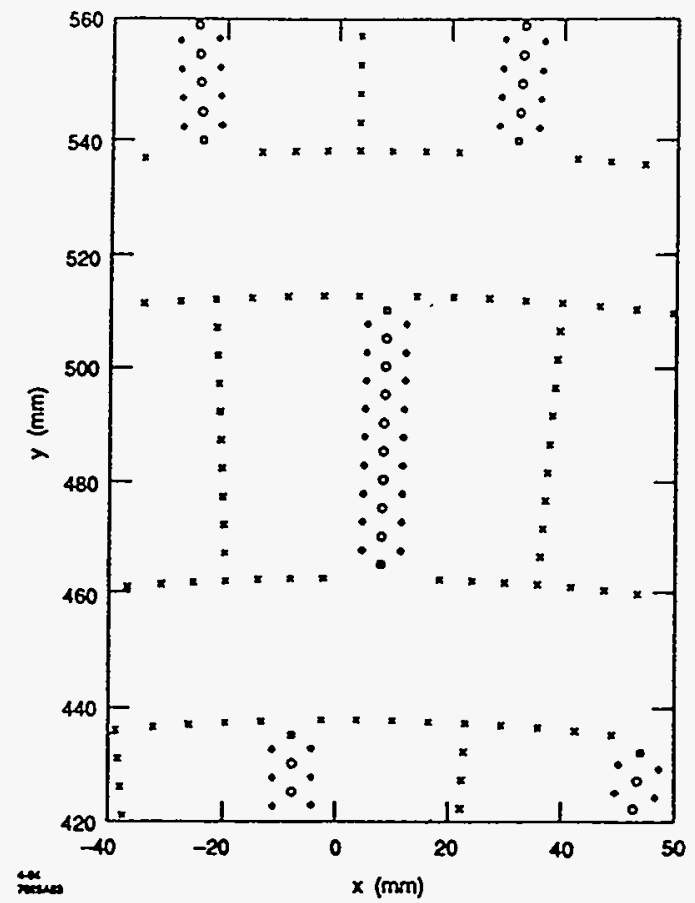

Figure 2.21: CDC drift cells. Circles: sense wires; Diamonds: guard wires; Crosses: field-shaping wires.

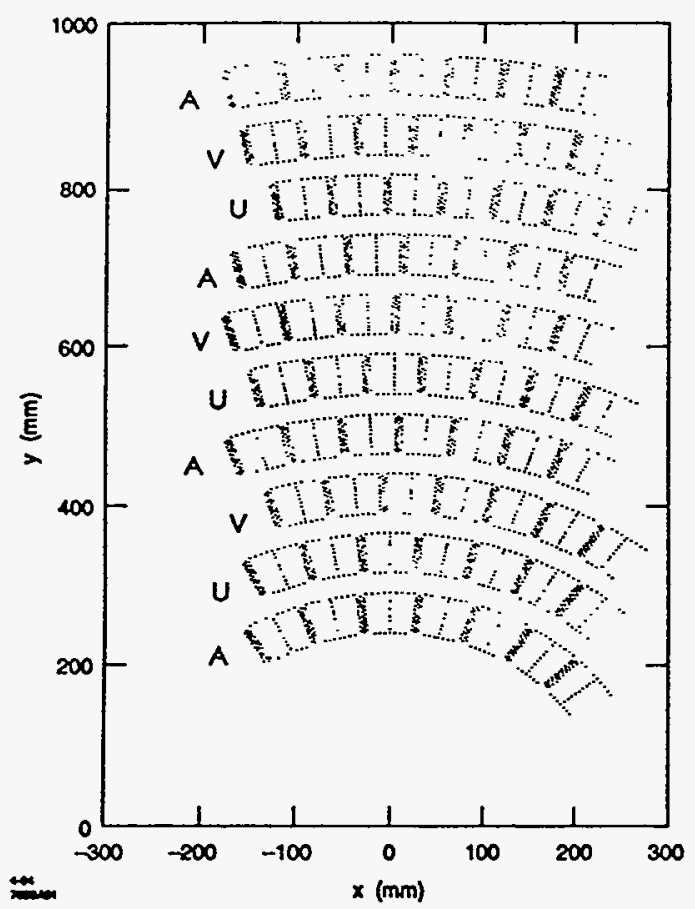

Figure 2.22: $\mathrm{CDC}$ superlayer structure. A denotes axial layers; $\mathrm{U}$ and $\mathrm{V}$ denote stereo layers. 


\subsubsection{Particle identification}

The Čerenkov ring imaging detector (CRID) was designed to allow particle identification in $Z$ decays, in particular to distinguish $\pi / \mathrm{K} / \mathrm{p}$ up to $30 \mathrm{GeV} / \mathrm{c}$ and $\mathrm{e} / \pi$ up to $6 \mathrm{GeV}$. The CRID consists of a barrel surrounding the CDC and an endcap between each EDC pair. A quadrant of the barrel CRID is shown in Fig. 2.23. A charged particle first passes through a $10 \mathrm{~mm}$ layer of $\mathrm{C}_{6} \mathrm{~F}_{14}$ liquid

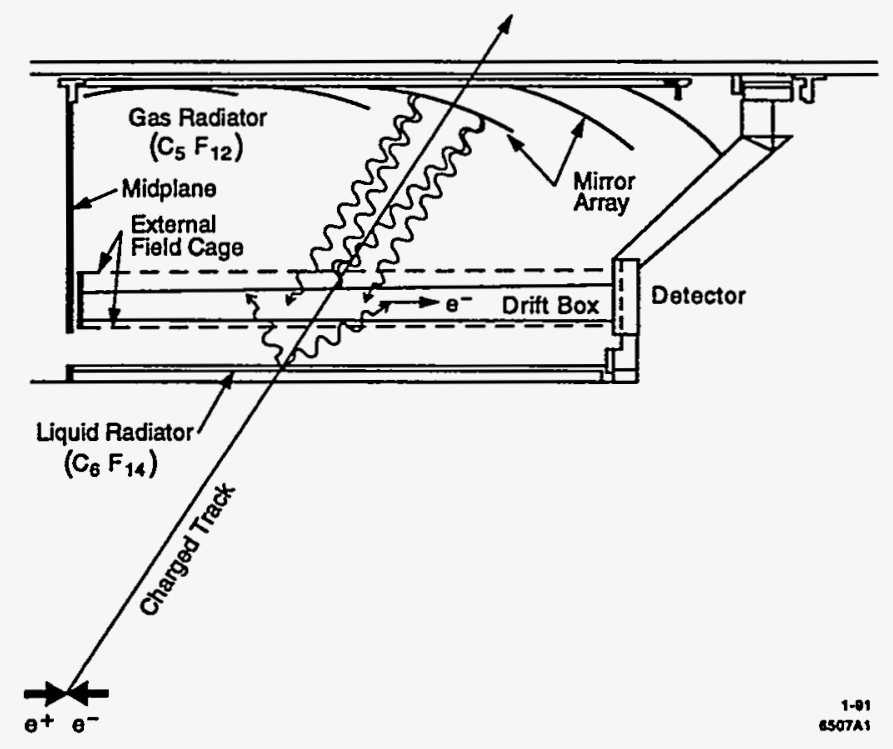

Figure 2.23: A quadrant of the barrel CRID.

radiator, giving a Čerenkov angle of $672 \mathrm{mr}$ for a $\beta=1$ particle. The produced Čerenkov cone then drifts $13 \mathrm{~cm}$ to a photon detector. The charged particle continues through the photon detector into a $45 \mathrm{~cm}$-thick $\mathrm{C}_{5} \mathrm{~F}_{12}$ gas radiator, where the resultant $59 \mathrm{mr}$ Cerenkov cone is reflected back from an imaging mirror array onto the other side of the photon detector. The detector is a time-projection drift box filled with TMAE-saturated ethane. The incident Cerenkov photons eject an electron from the TMAE which then drifts in an electric field to a sense-wire array at the end of the box. The drift time thus measures the $z$ coordinate of the incident Čerenkov photon while the sense wire measures the azimuthal position. In this way, the Cerenkov angle of traversing charged particles can be measured to $\sim 1 \mathrm{mr}$. 


\subsubsection{Calorimetry}

The SLD employs two calorimetry systems, the Liquid Argon Calorimeter (LAC) and the Warm Iron Calorimeter (WIC), to measure the electromagnetic and hadronic energy in tracks and hence to help distinguish $Z$ decay products. The LAC absorbs all electromagnetic energy and most of the hadronic energy in a track; the WIC detects the tails of the hadronic tracks and provides muon identification.

\section{Liquid argon calorimeter}

The LAC [41], shown in Fig. 2.19, is a parallel plate-liquid argon sampling calorimeter composed of a barrel calorimeter surrounding the barrel CRID and two endcap pieces outside the EDC. The barrel LAC is six meters long and has an inner and outer radius of $1.8 \mathrm{~m}$ and $2.9 \mathrm{~m}$, for a $\theta>33^{\circ}$ coverage. The endcaps fit inside the barrel LAC, covering $8^{\circ}<\theta<35^{\circ}$, and can be retracted for access to the detector. Together, the barrel and endcap LAC cover the region $|\cos \theta|<0.98$. The barrel LAC resides within the SLD solenoid, avoiding the resolution degradation suffered in traversing the coil material. A common volume of 35000 liters of liquid argon permeates the LAC, and cooling loops of 10000 liters/day of liquid nitrogen stabilize the liquid argon temperature.

The LAC is composed of 320 modules ( 288 in the barrel and 16 in each endcap), each of which in turn is made up of stacked parallel-plate liquid argon ionization chambers. The chambers consist of stacked lead tiles alternating with lead plates, separated by spacers, with liquid argon flowing between. The lead plates are grounded and each stack of tiles within a module is ganged together across the plates and held at high voltage to form the charge-collecting anodes. In this way, the absorber also serves as the electrodes, allowing a compact calorimeter design. The module geometry of the LAC is shown in Fig. 2.24.

The LAC is composed of two types of modules: electromagnetic (EM) and hadronic (HAD), with the HAD section mounted outside the EM section. In the EM calorimeter, the lead plates and tiles are $2 \mathrm{~mm}$ thick with a $2.75 \mathrm{~mm}$ spacing between for the liquid argon, providing $0.79 \mathrm{X}_{0} / \mathrm{cm}$ to traversing particles and a $\mathrm{dE} / \mathrm{dX}$ sampling fraction of $18 \%$. The EM calorimeter is divided into two radial sections, EM1 (six radiation lengths) and EM2 (fifteen radiation lengths). This total EM thickness contains $50 \mathrm{GeV}$ electrons with $1-2 \%$ energy leakage. The EM energy resolution is $\sim 15 \% / \sqrt{E}$. The HAD calorimeter is made up of $6 \mathrm{~mm}$ lead plates and tiles, separated by $2.75 \mathrm{~mm}$ of liquid argon. This geometry yields a density of $0.044 \lambda / \mathrm{cm}$ and a $\mathrm{dE} / \mathrm{dX}$ sampling fraction of $7 \%$. Each of the two HAD sections, HAD1 and HAD2, is 1 absorption length thick, giving a total EM + 


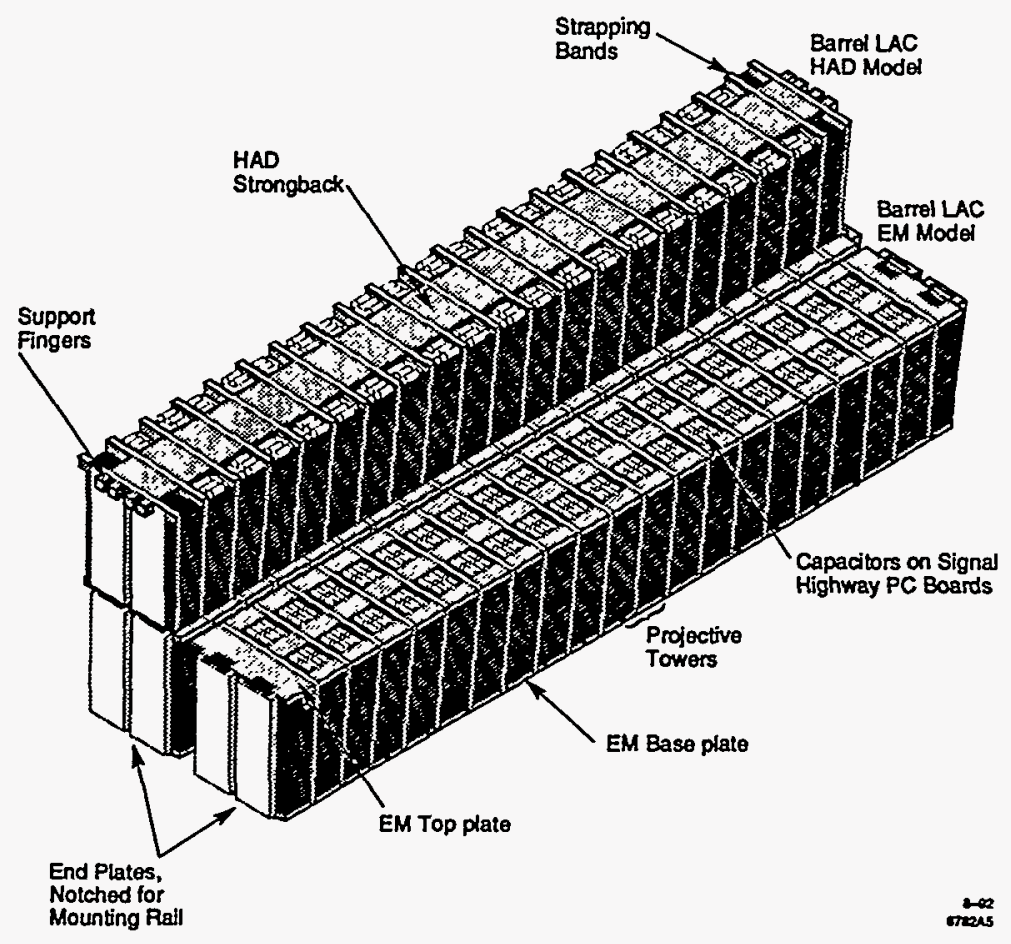

Figure 2.24: LAC barrel EM and HAD modules.

HAD thickness of 2.8 absorption lengths to measure $80-90 \%$ of the energy of a hadron shower. The $\mathrm{HAD}$ energy resolution is $\sim 65 \% / \sqrt{E}$ [42].

The spatial resolution of the LAC is determined by the tile size. The inside of the barrel is divided azimuthally into 192 sections, each subtending $33 \mathrm{mr}$ of azimuth, and is divided in polar angle into 68 sections of size from $21 \mathrm{mr}$ to $36 \mathrm{mr}$. The tile size increases toward the endcaps in order to provide a constant projective area for electromagnetic showers. HAD segmentation is twice as large in both transverse dimensions as in EM sections. A single projective unit of EM or HAD tile stacks is known as a "tower". Each endcap is segmented azimuthally into 192 EM sections at large radii, 96 sections at intermediate radii, and 48 sections at the center, maintaining an approximately constant projective area for electromagnetic showers. The endcaps are segmented into $17 \mathrm{EM}$ sections in polar angle. The HAD segmentation is again twice as large in the endcaps as the EM segmentation.

A number of innovative features have been implemented in the front-end LAC electronics. The electronics reside in units called "tophats" mounted directly on the liquid-argon cryostat. The tophat component schematic is shown in Fig. 2.25. Local amplification of the small LAC signals eliminates the need for low-level signal cables outside the cryostat. The tophat data is converted to light pulses and sent to a FASTBUS crate on top of the SLD via optical fiber. In order to reduce the heat load 


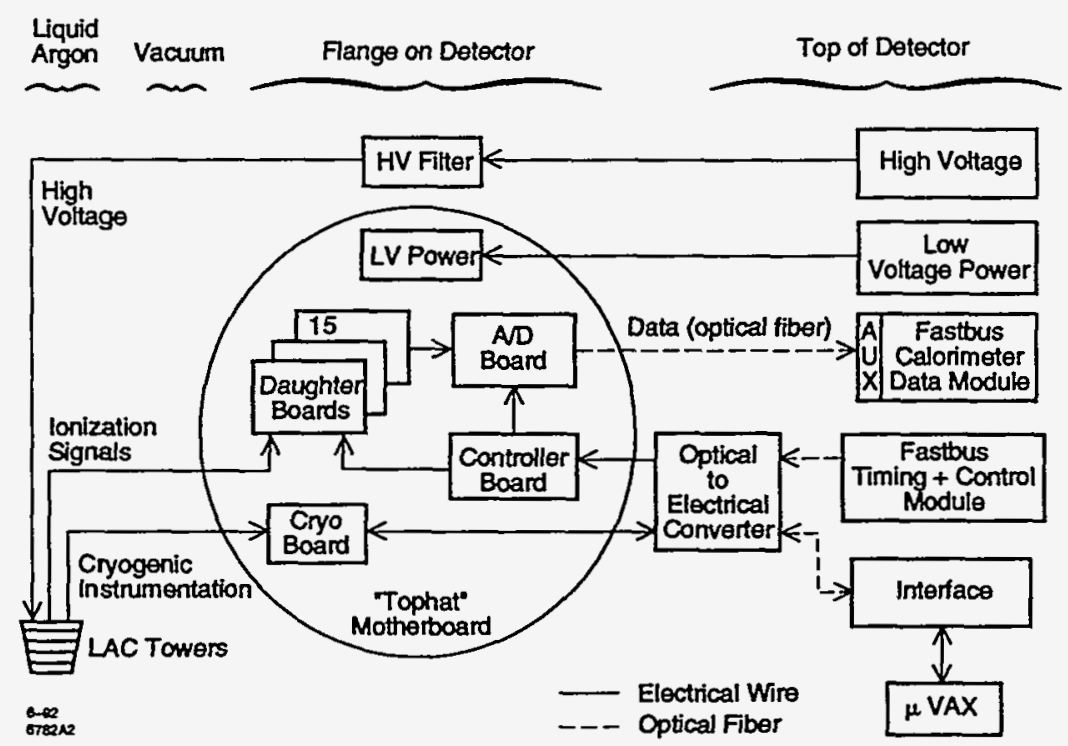

Figure 2.25: Schematic of LAC tophat and external connections.

from the tophats, the preamplifiers are turned off between the $120 \mathrm{~Hz}$ beam crossings, reducing the power consumption from $500 \mathrm{~W}$ per tophat to $60 \mathrm{~W}$.

Warm iron calorimeter

Like the LAC, the WIC [43] consists of a barrel calorimeter and two endcaps. The barrel WIC lies just outside the $0.6 \mathrm{~T}$ SLD solenoid and provides flux return for the magnet. In addition, it and the WIC endcaps are instrumented to measure the $10-20 \%$ hadronic energy leakage from the LAC and to provide muon tracking. The layout of the WIC is shown in Fig. 2.19; construction detail is shown in Fig. 2.26. The eight barrel sections of the WIC contain 14 layers of $50 \mathrm{~mm}$ iron with $32 \mathrm{~mm}$ gaps for instrumentation. The iron presents $>4$ interaction lengths to traversing particles, depending on direction, for a LAC/coil/WIC total of $>8$ interaction lengths. The fifteen gaps are filled with Iarocci streamer tubes laid out azimuthally for $r-\phi$ tracking. The $9 \mathrm{~mm} \times 9 \mathrm{~mm}$ graphite-coated plastic tubes contain a $100 \mu \mathrm{m} \mathrm{BeCu}$ wire at $4.5 \mathrm{kV}$ and a $88 \% \mathrm{CO}_{2} / 9.5 \%$ Isobutane $/ 2.5 \% \mathrm{Ar}$ gas mixture. Each set of tubes faces on one side a copper pad and on the other copper strips laid out along the tubes. Traversing particles create an avalanche that travels to the wire, leaving an ion cloud that induces charge proportional to the number of tracks on the strips and pads. In addition, two of the instrumented layers contain strips perpendicular to the streamer tubes, providing two $z$ coordinates for muon tracking to $\leq 10 \mathrm{mr}$. The endcap WIC contains eight vertical and eight horizontal layers 


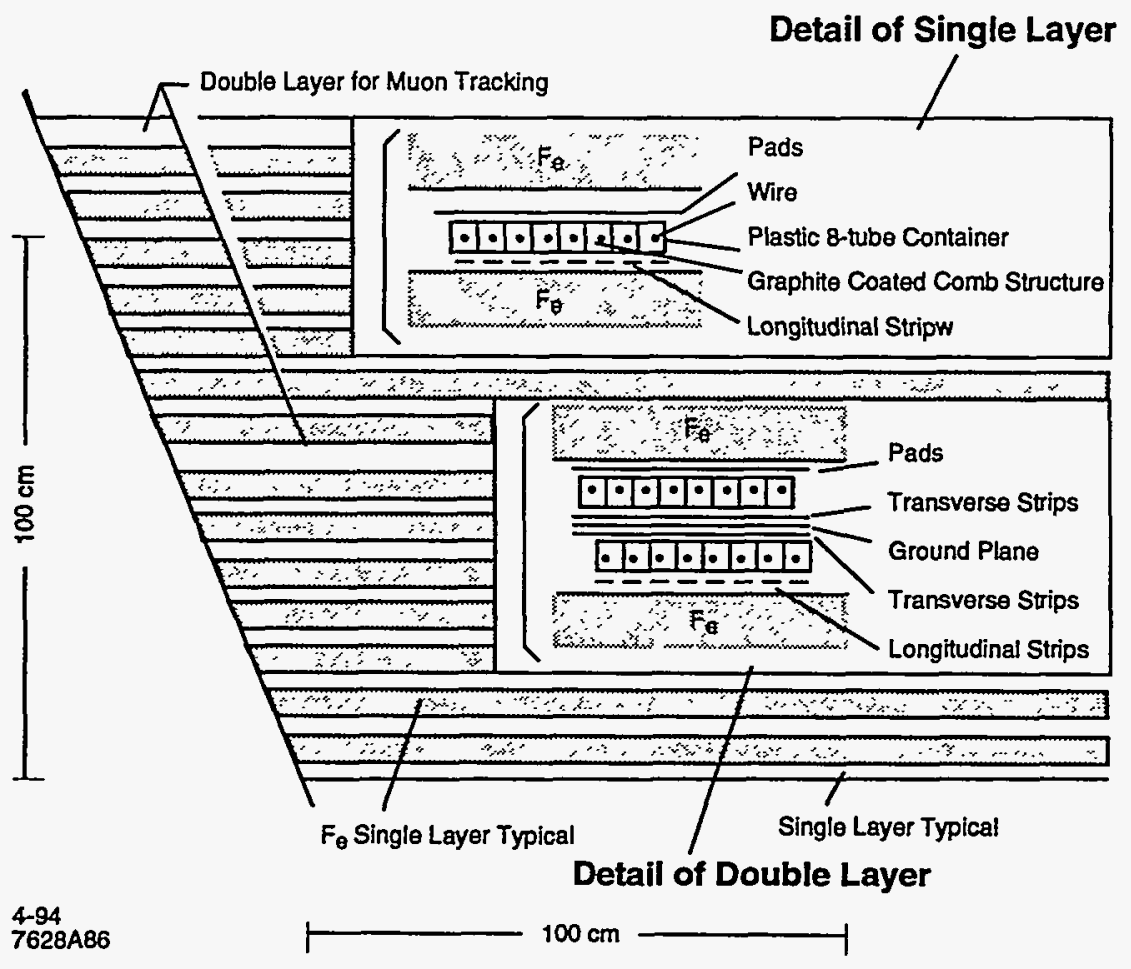

Figure 2.26: Section detail of the WIC.

of tubes. The pad layout in both WIC barrel and endcaps follows the tower structure in the LAC, the pad size increasing outward from $265 \mathrm{~mm} \times 216 \mathrm{~mm}$ to $295 \mathrm{~mm} \times 316 \mathrm{~mm}$ in the barrel. The pads of the inner and outer halves of layers are ganged together to form two radial towers. The energy resolution in the WIC is $\sim 0.8 / \sqrt{E}(\mathrm{GeV})$, for a combined LAC/WIC energy resolution of $\sim 0.5 / \sqrt{E}(\mathrm{GeV})$.

\subsubsection{Luminosity monitoring/small-angle detection}

The luminosity determination at SLD is performed by measuring the rate of $t$-channel Bhabha events into a known acceptance at small polar angle. This measurement is made by the luminosity monitor/small angle tagger (LMSAT) [44], shown in Fig. 2.27. Also shown is the medium angle silicon calorimeter (MASC), used to extend the electromagnetic coverage of SLD to small angles. Both components shown have matching complements opposite the IP. The LMSAT, a silicon sampling calorimeter with tungsten radiator at a distance of $100 \mathrm{~cm}$ from the IP, covers the region from 23 to $68 \mathrm{mr}$ in polar angle. It consists of 23 tungsten plates of $3.5 \mathrm{~mm}$ thickness each, spaced $4.5 \mathrm{~mm}$ apart, for a total of 21 radiation lengths (>99.5\% of a $45 \mathrm{GeV}$ electromagnetic shower). 


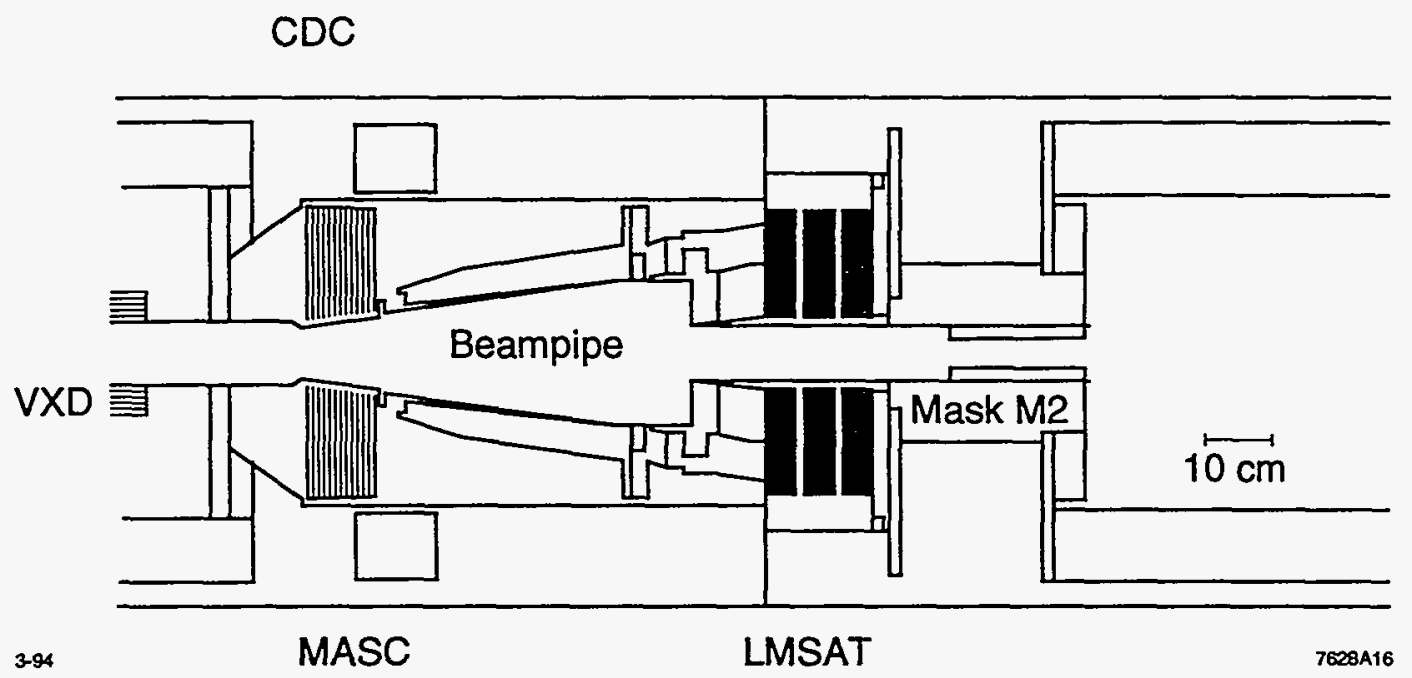

Figure 2.27: The LMSAT and MASC small angle calorimeters.

The instrumentation is provided by interleaved silicon detectors segmented transversely into $\sim 1 \mathrm{~cm}^{2}$ cells. As in the LAC and WIC, projective towers are formed by connecting successive cells in the first six layers and in the remaining 17 layers. The MASC, lying $31 \mathrm{~cm}$ from the IP, extends from 68 to $190 \mathrm{mr}$ and consists of $106.6 \mathrm{~mm}$-thick tungsten plates sandwiched with segmented silicon detectors. The MASC is similarly divided into towers, and the entire LMSAT/MASC system produces a total of 1024 signals. The energy resolution of the LMSAT is $\sim 3.5 \%$ at $50 \mathrm{GeV}$, and the angular resolution is $\sim 200-300 \mu \mathrm{r}$. 


\section{Chapter 3}

\section{Polarization Measurement}

The most relevant and interesting topic in the measurement of $A_{L R}$ is the polarization determination. The beam polarization measurement contributes the dominant systematic error in the experiment and hence requires much care and precision in execution. Ultimately, with several hundred thousand $Z$ s at high polarization, $\mathcal{P}^{e}$ must be determined to $\sim 1 \%$ of itself.

\subsection{Compton Polarimetry}

Doubly polarized Compton scattering, as discussed in the previous chapter, provides a precise beam polarization measurement. Detection of the scattered electrons, as is done at SLC, offers the advantages of a large experimental asymmetry and spatial separation of the kinematic spectrum.

\subsubsection{Compton scattering}

Tree-level Compton scattering proceeds through the $t$ - and $s$-channel diagrams shown in Fig. 3.1. The Compton-scattering cross section is most often derived in the center-of-mass frame or the rest frame of the electron. Unfortunately, in both of these frames, the total energy of the SLC Compton $e^{-}+\gamma$ is less than $1 \mathrm{MeV}$, and hence the electron mass cannot be neglected to simplify the formalism. The expression for the differential cross section in the electron rest frame is [45]

$$
\left(\frac{d \sigma}{d \Omega}\right)=\frac{1}{2} \tau_{\circ}^{2}\left(\frac{k^{\prime}}{k}\right)\left[\frac{\left(k-k^{\prime}\right)^{2}}{k k^{\prime}}+1+\cos ^{2} \theta_{\circ}\right]\left\{1-\mathcal{P}^{\gamma} \mathcal{P}^{e} A^{e \gamma}\left(\vec{k}, \vec{k}^{\prime}\right)\right\}
$$




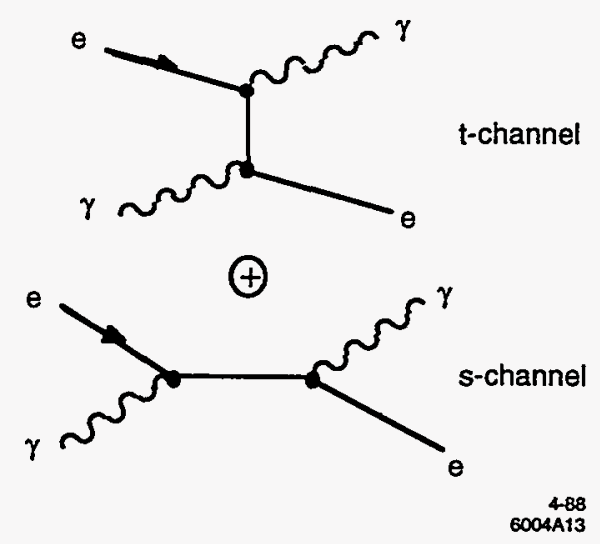

Figure 3.1: Tree-level Compton-scattering diagrams.

where $r_{\circ}=2.82 \times 10^{-13} \mathrm{~cm}$ is the classical radius of the electron; $\vec{k}$ and $\vec{k}^{\prime}$ are the incident and scattered photon momenta; $\theta_{0}$ is the photon scattering angle with respect to the incident photon direction; $\mathcal{P}^{\gamma}$ is the signed circular polarization of the photon, where $\mathcal{P}^{\gamma}>0$ for photon spin along the momentum direction; $\mathcal{P}^{e}$ is the electron polarization; and $A^{e \gamma}\left(\vec{k}, \vec{k}^{\prime}\right)$ is the Compton polarization asymmetry function given by

$$
A^{e \gamma}\left(\vec{k}, \vec{k}^{\prime}\right)=\frac{\left(\frac{1}{k^{\prime}}-\frac{1}{k}\right)\left[\vec{k} \cos \theta_{\circ}+\vec{k}^{\prime}\right] \cdot \hat{s}}{\frac{\left(k-k^{\prime}\right)^{2}}{k k^{\prime}}+1+\cos ^{2} \theta_{\circ}},
$$

where $\hat{s}$ is the electron polarization direction. The signs in the asymmetry term $\mathcal{P}^{\gamma} \mathcal{P}^{e} A^{e \gamma}$ have been chosen so that the Compton cross section is greater for the case of parallel incident photon and electron spins; this result is developed in the final chapter.

Our purposes require an expression in the SLC laboratory frame, where the electron momentum is $2 \times 10^{10}$ times that of the photon. In this case, the scattered photons and electrons travel in the incident electron direction. We define the lab-frame incident and final electron energies and incident and final photon energies as $E, E^{\prime}, K$, and $K^{\prime}$; then with the kinematical variable $y$ defined as

$$
y \equiv\left(1+\frac{4 E K}{m^{2}}\right)^{-1}
$$

we can write

$$
\begin{aligned}
K_{\max }^{\prime} & =E(1-y) \\
E_{\min }^{\prime} & =E y
\end{aligned}
$$


for the final photon and electron energies corresponding to complete backscattering in the lab frame. For the SLC Compton values of $2.33 \mathrm{eV}$ and $45.6 \mathrm{GeV}$ for the photon and electron energies, $y=0.381$, giving a maximum scattered photon energy of $28.3 \mathrm{GeV}$ and a minimum electron energy of $17.4 \mathrm{GeV}$. In addition, the angle $\theta_{K}$ of the scattered photon is given by

$$
K^{\prime}=K_{\max }^{\prime}\left[1+y\left(\frac{E \theta_{K}}{m}\right)^{2}\right]^{-1} \equiv K_{\max }^{\prime} \cdot x
$$

where $m$ is the electron mass. The width of the high-energy photon angular distribution at SLC is a few tens of microradians, which is smaller than the beam divergence; also, the maximum electron scattering angle $\theta_{e}^{\max }$ is given by

$$
\theta_{e}^{\max }=\frac{m_{e}}{2 E} \frac{1-y}{y}=9.1 \mu \mathrm{r}
$$

Therefore, the scattered photons and electrons remain within the unscattered beam. To obtain the Compton cross section in the laboratory frame, we use the following transformation:

$$
\begin{aligned}
K & =\frac{m}{2 E} k \\
x & =\frac{K^{\prime}}{K_{\max }^{\prime}} 1-\cos \theta_{0} \\
& =\frac{\left.1-\cos \theta_{\circ}\right)}{2 y+(1-y)(1-}
\end{aligned}
$$

The resulting expression in the lab frame is $[46,47]$

$$
\left(\frac{d^{2} \sigma}{d x d \phi}\right)_{C o m p}=\left(\frac{d^{2} \sigma}{d x d \phi}\right)_{u n p o l}\left\{1-\mathcal{P}^{\gamma}\left[\mathcal{P}_{z}^{e} A_{z}^{e \gamma}(x)+\mathcal{P}_{t}^{e} \cos \phi A_{t}^{e \gamma}(x)\right]\right\}
$$

where $\phi$ is the azimuth of the photon with respect to the electron transverse polarization, the unpolarized cross section is given by

$$
\left(\frac{d^{2} \sigma}{d x d \phi}\right)_{\text {unpol }}=r_{\circ}^{2} y\left\{\frac{x^{2}(1-y)^{2}}{1-x(1-y)}+1+\left[\frac{1-x(1+y)}{1-x(1-y)}\right]^{2}\right\},
$$

and the longitudinal and transverse asymmetries are defined as

$$
A_{z}^{e \gamma}(x)=r_{\mathrm{o}}^{2} y[1-x(1+y)]\left\{1-\frac{1}{[1-x(1-y)]^{2}}\right\} \cdot\left(\frac{d^{2} \sigma}{d x d \phi}\right)_{\text {unpol }}^{-1}
$$




$$
A_{t}^{e \gamma}(x)=r_{\circ}^{2} y x(1-y) \frac{[4 x y(1-x)]^{1 / 2}}{1-x(1-y)} \cdot\left(\frac{d^{2} \sigma}{d x d \phi}\right)_{\text {unpol }}^{-1}
$$

The polarizations $\mathcal{P}_{z}^{e}$ and $\mathcal{P}^{\gamma}$ in Eq. 3.5 must both be considered signed, so that both are positive for spin direction along the momentum vector. This convention again yields a greater cross section for parallel incident electron and photon spins. These equations are shown in Fig. 3.2 for the photon $(2.33 \mathrm{eV})$ and electron $(46 \mathrm{GeV})$ energies used at the SLC. The longitudinal asymmetry has a

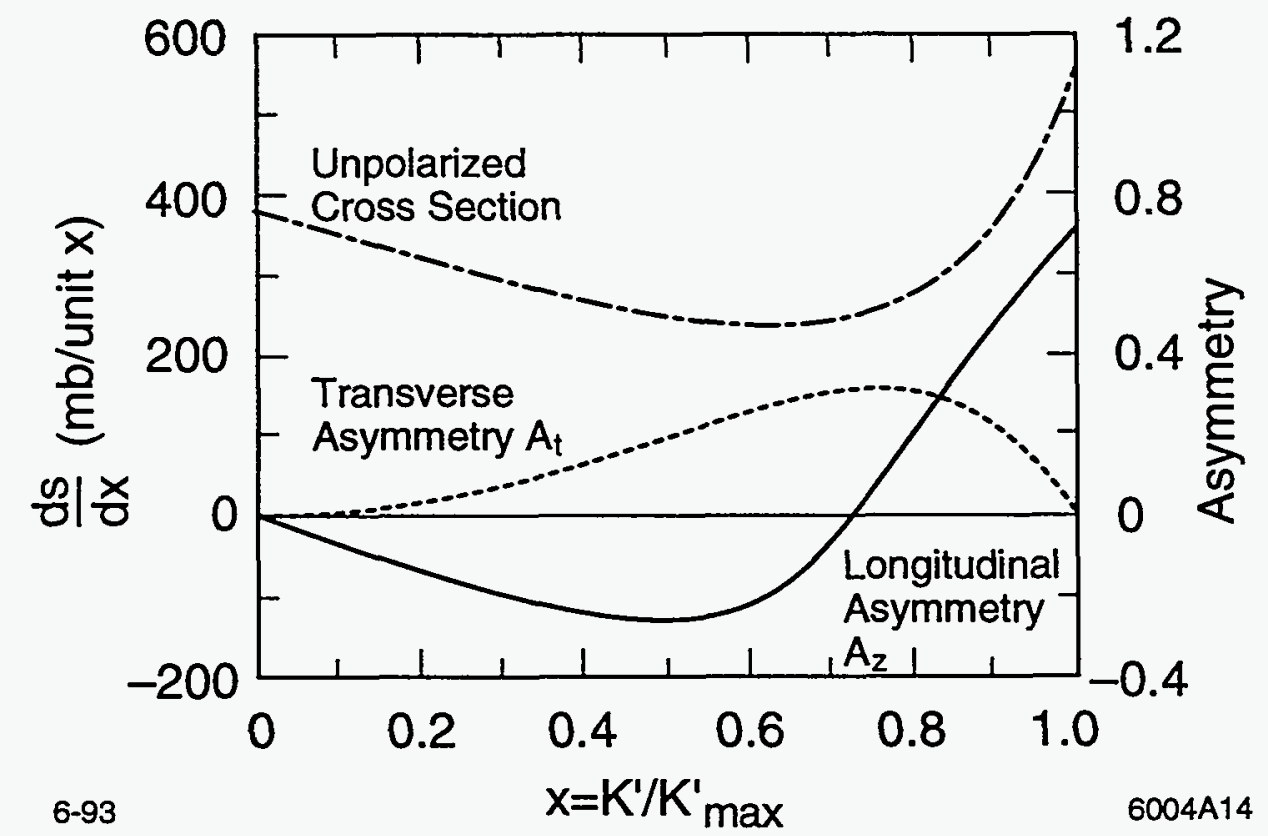

Figure 3.2: The unpolarized cross section and longitudinal and transverse asymmetries for Compton scattering of a $2.33 \mathrm{eV}$ photon with a $46 \mathrm{GeV}$ electron.

maximum of $\sim 75 \%$ at the minimum scattered electron energy (backscattered case), passes through zero at $x=1 / 1+y\left(25.16 \mathrm{GeV}\right.$ Compton $e^{-}$energy at SLC), and becomes negative at small energy transfers. At the SLC, the Compton analyzing dipoles convert the energy dependence into a function of position, and hence the inaccessible kinematic region beyond $x=1$ results in a "Compton edge," which is observable in the Čerenkov detector and is an invaluable calibration feature.

First-order radiative corrections to polarized Compton scattering at the SLC electron and photon energies have been calculated [48]. The effect on the unpolarized differential cross section is $<0.3 \%$ and the correction to the Compton asymmetry function is a shift of $\left|\Delta A_{z}^{e \gamma}\right|<0.0006$ for all scattered electron energies detected at SLC. Therefore, we neglect radiative corrections in the analysis. 


\subsubsection{Experimental asymmetry}

As is evident from Eq. 3.5, the longitudinal electron beam polarization dependence manifests itself in a cross section asymmetry (Eq. 3.7) between parallel and antiparallel photon and electron spin combinations. Therefore, we form an experimental asymmetry between these states in the Cerenkov detector to extract $\mathcal{P}^{e}$. The total signal $N_{\text {anii }}^{\text {par }}$ per parallel- or antiparallel-spin photon-electron beam crossing in the $i^{\text {th }}$ Cerenkov channel is given by

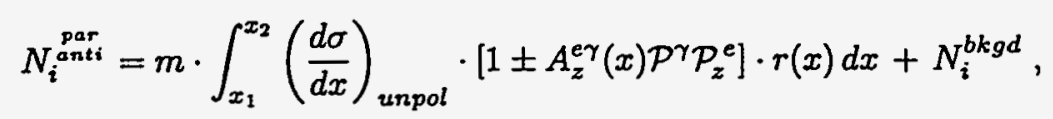

where $m$ is the Compton luminosity per beam crossing, $x_{1}$ and $x_{2}$ are the channel energy limits, and $r(x)$ is the Čerenkov channel response function. In each channel, we form the following experimental asymmetry with the data averaged over each run:

$$
\begin{aligned}
\text { Exp. asym. }_{\cdot i} & =\frac{\langle N\rangle_{i}^{p a r}-\langle N\rangle_{i}^{a n t i}}{\langle N\rangle_{i}^{p a r}+\langle N\rangle_{i}^{a n t i}-2 \cdot\langle N\rangle_{i}^{b k g d}} \\
& =\mathcal{P}^{r} \cdot \mathcal{P}_{z}^{e} \cdot a_{i}
\end{aligned}
$$

where $a_{i}$, the analyzing power of the Cerenkov channel, is defined as

$$
a_{i} \equiv \frac{\int_{x_{1}}^{x_{2}}\left(\frac{d \sigma}{d x}\right)_{u n p o l} A_{z}^{e \gamma}(x) r(x) d x}{\int_{x_{1}}^{x_{2}}\left(\frac{d \sigma}{d x}\right)_{\text {unpol }} r(x) d x},
$$

and hence the electron polarization is simply extracted:

$$
\mathcal{P}_{z}^{e}=\frac{E x p . a s y m_{\cdot i}}{\mathcal{P}^{\gamma} \cdot a_{i}}
$$

The measurement of the electron polarization thus depends on the measured experimental asymmetry in a channel, the calculated analyzing power in that channel, and the measured photon beam circular polarization. Clearly, $\mathcal{P}_{z}^{e}$ can be calculated using any Cerenkov channel once the position of the detector is calibrated (all channels must agree); however, the advantage of using the outermost channels is that they have larger asymmetries and analyzing powers, implying smaller fractional error in measurement and calculation. 


\subsection{Monte Carlo Simulation}

We have seen in the previous section that the determination of the electron polarization depends upon the calculated analyzing power of a Čerenkov channel, which in turns depends upon the Compton cross section and asymmetry function and the channel response function, convoluted and integrated over the channel limits. Therefore, it is crucial to understand both the detector position with respect to the Compton spectrum and the signal produced in the channel by a uniform electron flux. As a high-quality electron test beam is not available for measurements of the latter, the detector response must be evaluated with a Monte Carlo model.

\subsubsection{The EGS4 Monte Carlo program}

The natural choice for detailed modeling of detector response to high-energy electrons is the EGS4 Monte Carlo program [49]. EGS (Electron-Gamma Shower) simulates the interaction of electrons, positrons and photons with matter over a large range of energies ( $\sim 10 \mathrm{keV} \sim 1 \mathrm{TeV})$. The program properly takes into account photoelectric, Compton, and pair-production interactions, as well as bremsstrahlung, Molière multiple scattering, Møller and Bhabha scattering, and $e^{+}$annihilation at rest and in flight. EGS recognizes all elements and most commonly used metal alloys and composite materials. The EGS program is a world-wide standard and has been used in innumerable physics experiments over the years.

\subsection{2 Čerenkov detector modeling}

The Cerenkov detector simulation consists of three stages: formation of the Compton-scattered electron beam; modeling of the detector geometry; and Čerenkov photon production and transport to the phototubes.

\section{Beam modeling}

The detector simulation picks electrons at random from a modeled Compton-scattered electron beam starting at the effective center-of-bend point of the Compton dipole magnets. The electrons are given an initial deviation, random in size and direction, from the center of the beam according to the expected $190 \times 10^{-4} \mathrm{~cm}$ gaussian spot size at the center-of-bend point. In addition, the electrons are given a polar angle, again random in size and azimuth, according to their $50 \mu \mathrm{r}$ divergence [50]. The energy, and hence tranverse bend, of the electrons is chosen randomly from a flat distribution 
and added to the transverse component of the divergence angle to determine the final trajectory. A flat energy distribution is used in order to obtain a cross section-independent detector response function.

\section{Detector geometry}

The purpose of the detector simulation is to model the important geometrical features and materials as accurately as possible for a given Cerenkov channel. The front section of the detector is shown in Fig. 3.3, and general design information was given in the previous chapter. The salient features

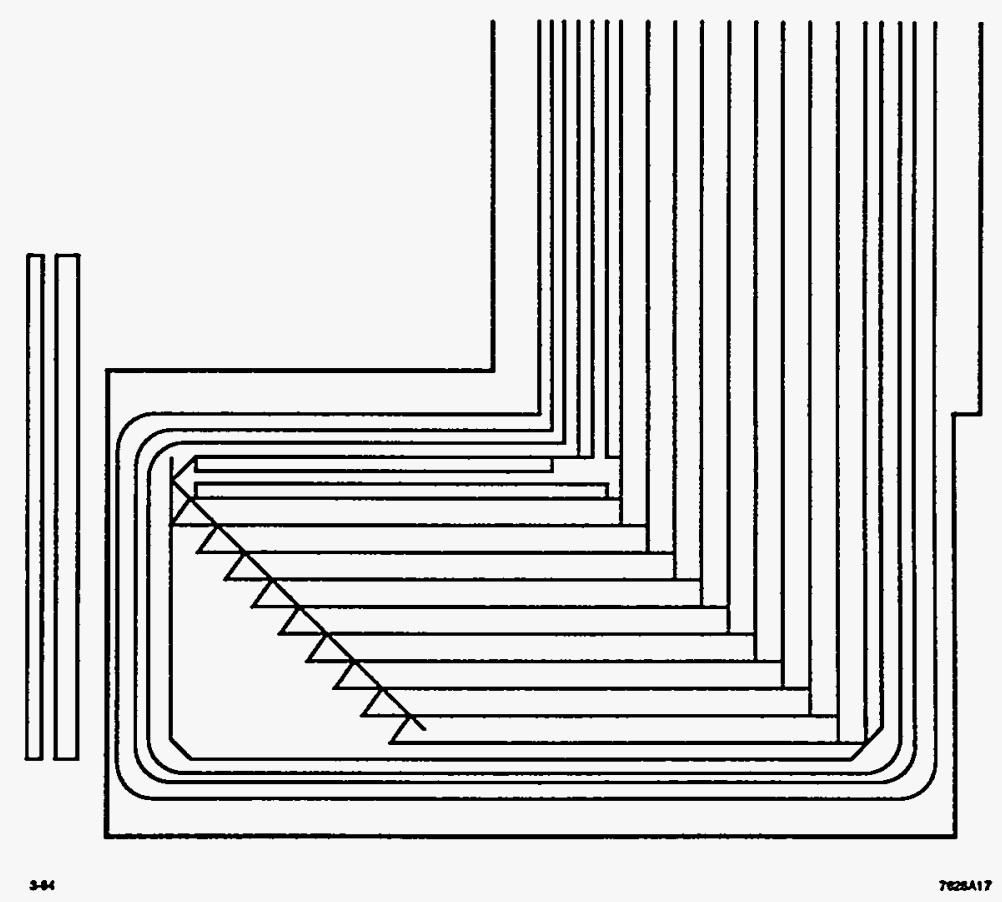

Figure 3.3: Construction drawing of the front section of the Čerenkov detector.

included in the model are the lead preradiator/shielding in front (not shown in the figure), the aluminum front detector wall, ceiling, and floor, and aluminum channel walls. The channels begin at different $z$ positions, to accommodate the transverse bend section, and are projective in the radiator section, with a wedge angle of $2.932 \mathrm{mr}$. In order to maintain a constant acceptance for each channel, the channels must all have slightly different initial widths due to their different initial $z$ positions. The design specifies that the central channel, channel 5 , have a width of $1.000 \mathrm{~cm}$ between 
walls at its front, and all channels have the same width at any fixed $z$ position. The $z$ distance from the effective bend point to the front of channel 5 has been measured to be $355.8 \pm 1 \mathrm{~cm}$. The front of each channel is practically defined by the sloping aluminum tab blocking initial Čerenkov radiation; the simulation assumes a straight line at the average $z$ position of the start of the two channel walls. The error in the calculated analyzing powers due to this simplification has been studied and found to be negligible. In addition, only walls of the channel under study are included in the simulation. The effects due to this omission have also been studied and found to be unobservable. The simulation neglects all detector elements downstream of the radiator section. The Monte Carlo allows rotation of the detector channel in the $x-z$ plane in order to study systematic effects. The detector has been surveyed to within a few milliradians of correct angular alignment, defined as the detector orientation at which the central axis of channel 5 coincides with the Compton electron ray striking the center of channel 5 at the front of the channel. Correct alignment is assumed in the Monte Carlo.

\section{Čerenkov light generation and transport}

Charged shower products emerging from the lead shield traverse the detector channels and create Cerenkov radiation, which bounces down the walls to the phototubes. The detector characteristics relevant to generation, transport, and detection of the Čerenkov light are the gas threshold, reflectivity of the channel, and phototube quantum efficiency.

The threshold energy of the cis- and trans-2-butene Čerenkov gas used is $10.2 \mathrm{MeV}$ at wavelengths of 436-644 nm [51]. However, the sensitive range of the phototubes extends to shorter wavelengths $(160-650 \mathrm{~nm})$, and data on the index of refraction at these wavelengths has not been found. Attempts to deduce the index of refraction from the measured absorption at the shorter wavelengths $[52,53]$ using the dispersion relations are fruitless due to large disagreements between the measurements. Hence, we use the $10.2 \mathrm{MeV}$ value in the simulation with the knowledge that it may be somewhat lower on average due to the fact that the absorption resonance occurs within the phototube response limits [52, 53]. The threshold energy has been varied in the Monte Carlo between $5 \mathrm{MeV}$ and $20 \mathrm{MeV}$; no significant effect is seen in the calculated analyzing powers.

A $10.2 \mathrm{MeV}$ threshold implies that the maximum Cerenkov angle is $50 \mathrm{mr}$ for highly relativistic particles and that the light yield is $\sim 5$ photons/cm (within the PMT response) in the detector. As charged particles pass through the active region of the simulated channel, they create photons with the following characteristics:

- The number of photons is determined by the length of the passage through the channel and 
the energy of the particle;

- The photons are emitted randomly in travel distance through the channel;

- The energy of the photons is randomly chosen from a flat distribution within PMT response limits;

- The polar angle of the photon with respect to the charged particle is determined according to the particle's energy, and the azimuth is random.

The direction of each photon with respect to the channel axis is then calculated. The transport efficiency of the photons through the Čerenkov detector is determined using a lookup table generated by a separate program that considers polar and azimuthal angle, energy, and starting position of the photon, and uses the channel dimensions and the known Fresnel coefficients for reflection from aluminum to calculate the probability of delivery to the phototube. The program also convolutes the phototube quantum efficiency spectrum to find the signal, in photoelectrons, per photon and hence per traversing charged particle. The average transmission through the detector is decreased slightly to agree with lab data for the $55 \%$ transmission of $2537 \mathrm{~nm}$ light at $50 \mathrm{mr}$ incident angle, as absorptive imperfections and cracks exist in the detector channels. Absorption in the Cerenkov gas, however, is likely a bigger, but unknown, factor. In any case, knowledge of the overall gain of the detector is unnecessary for measurement of the asymmetry.

An example of the showering of a single Compton electron in a Čerenkov channel is given in Figs. 3.4, 3.5, and 3.6. The figures show three views of a $28 \mathrm{GeV}$ electron traversing the axis of Čerenkov channel 1 with $7.6 \mathrm{~mm} \mathrm{~Pb}$ shielding. The lead clearly has a large effect on the channel response.

\subsection{3 Čerenkov response functions}

The detector simulation program histograms the average response and uncertainty of the detector to incident Compton electrons as a function of horizontal striking position at the front of the channel. This position scale (transverse distance from the undeflected, on-energy beamline, i.e., the pre-bend electron axis) is one-to-one related to the kinematic energy variable $x$, up to finite spot size, energy spread, and divergence effects, and is more closely related to the experimental situation. Hence, the response functions and analyzing powers are calculated with this position variable.

An example of an EGS-simulated response function for Čerenkov channel 6 with no lead shield is given in Fig. 3.7. The response is close to the ideal step function, with flat response for electrons 


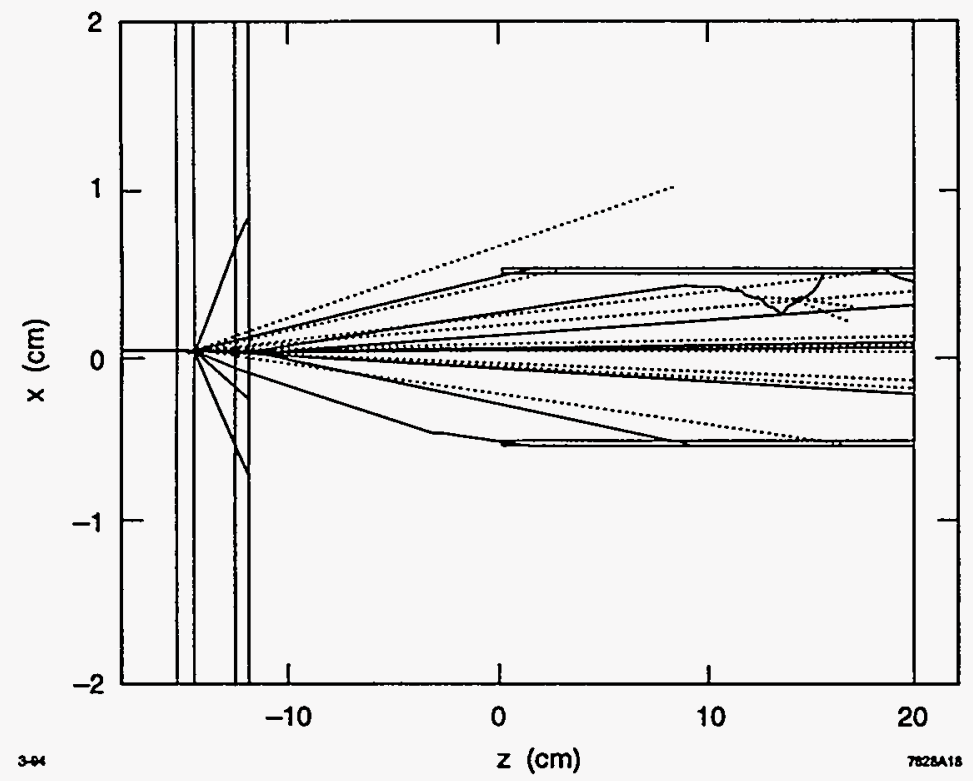

Figure 3.4: Passage of a $28 \mathrm{GeV}$ electron through Čerenkov channel 1 as viewed from above. The lead shield is on the left, followed by the front wall of the detector. Charged particles are shown with solid lines, photons with dotted lines.

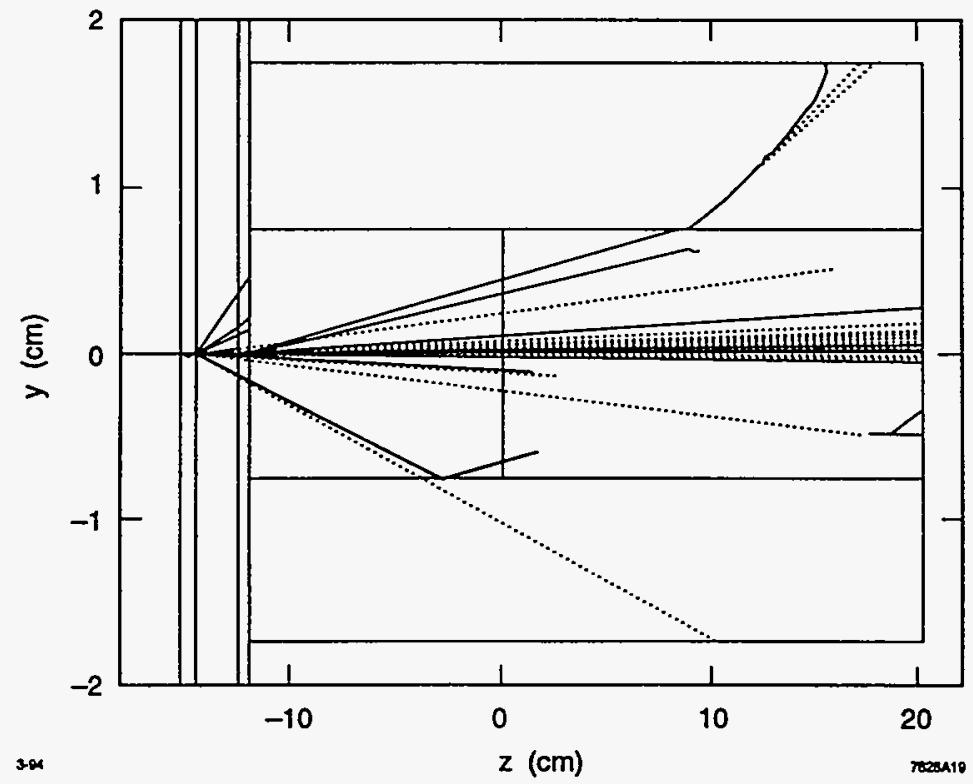

Figure 3.5: The same interaction viewed from the side. 


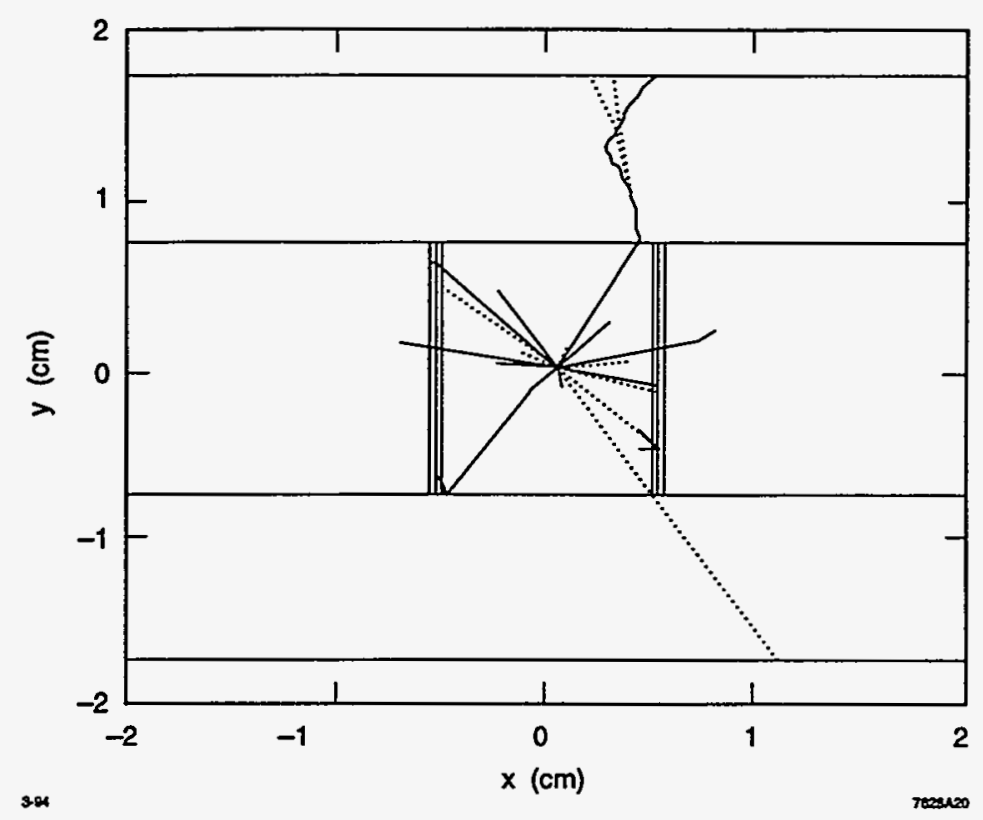

Figure 3.6: Beam's eye view of the interaction.

striking inside the channel and no response for electrons striking outside. The sharp "ears" are due to showering in the $250 \mu \mathrm{m}$ projective aluminum walls. The ears cause a completely negligible correction to the analyzing power of the channel, demonstrating that if no shielding were necessary, simulation could have been avoided altogether. Fig. 3.8 shows the effect of adding $7.6 \mathrm{~mm} \mathrm{~Pb}$ shielding, the standard amount during the run. The showering increases by many times, the response is no longer flat in the center of the channel, and long tails appear on either side, evidence of smearing from adjacent channels. Clearly, the resolution is greatly affected by the lead, and use of the Monte Carlo becomes justified. An absorption-independent check on the signal multiplication with lead is possible by comparing the ratio of these integrated signals with the same experimental ratio. The Monte Carlo gives a multiplication of 4.6; the data, which is minimal without lead, gives a ratio of 4-5. It is the shape of the response function, however, that determines its effect on the analyzing power and detector calibration. A study of the response function shape will be presented in the next section.

The shapes of the Compton asymmetry and unpolarized cross section are shown in Fig. 3.9. The final calibrated positions of the spectra are plotted, as well as the positions of the first seven Cerenkov channels (channels 8 and 9 lie beyond the Compton edge), and their calculated analyzing powers. The Compton edge lands inside channel 7 and the zero-asymmetry point falls inside channel 2 . We see that the effects of smearing and cross section shape on the analyzing powers are calculated to 


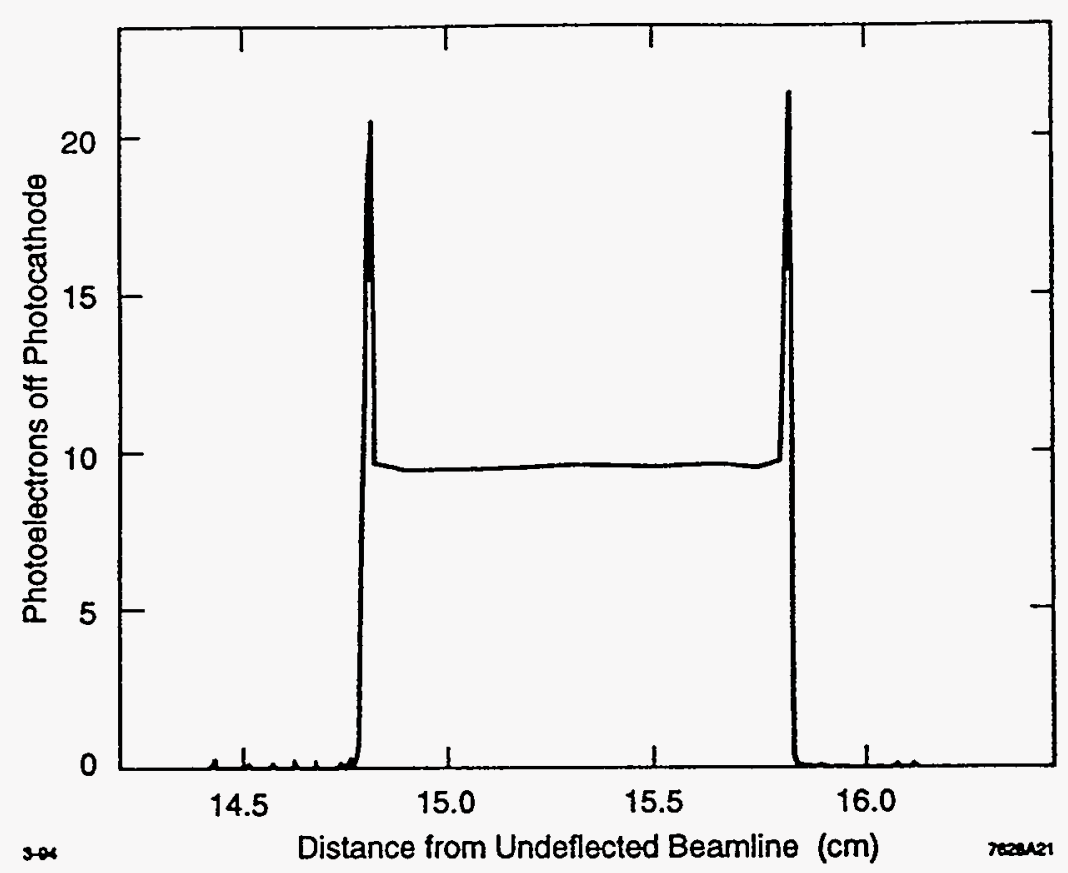

Figure 3.7: EGS-derived response function for Čerenkov channel 6 with no $\mathrm{Pb}$ shield.

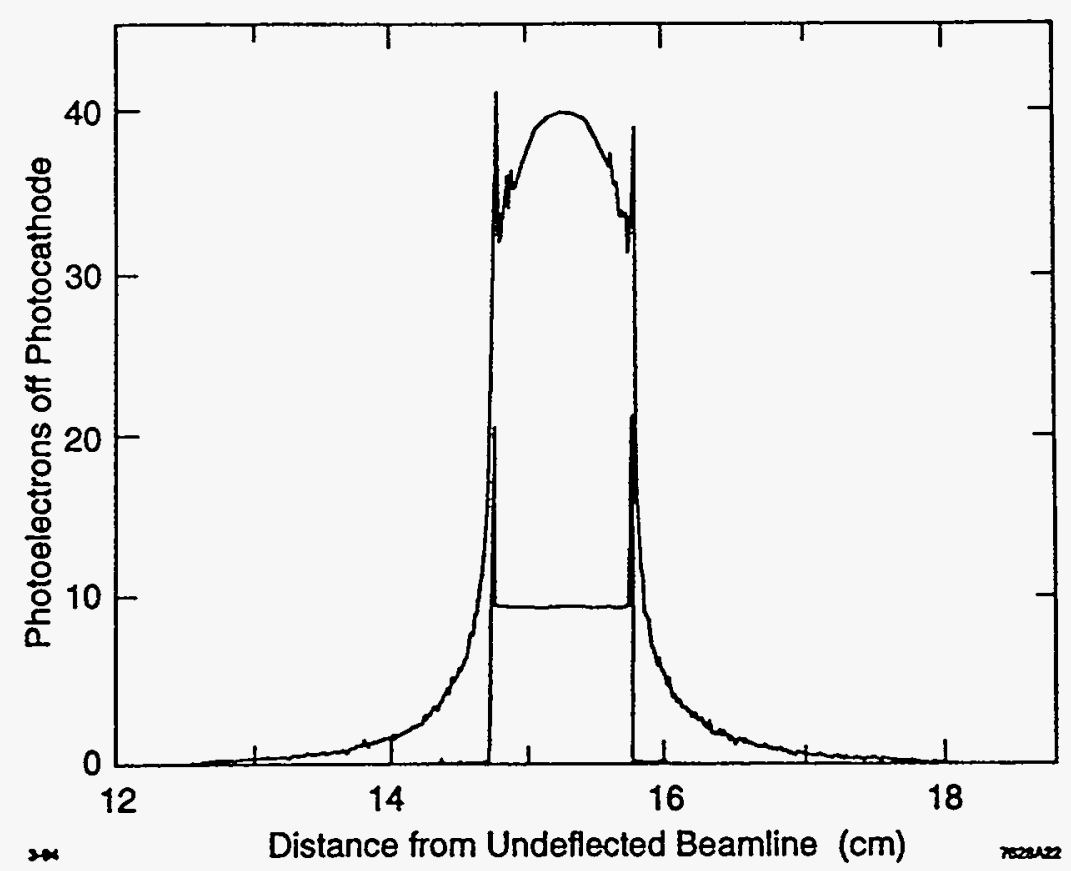

Figure 3.8: Response function for channel 6 with $7.6 \mathrm{~mm} \mathrm{~Pb}$ shielding. The upper plot is inlaid. 


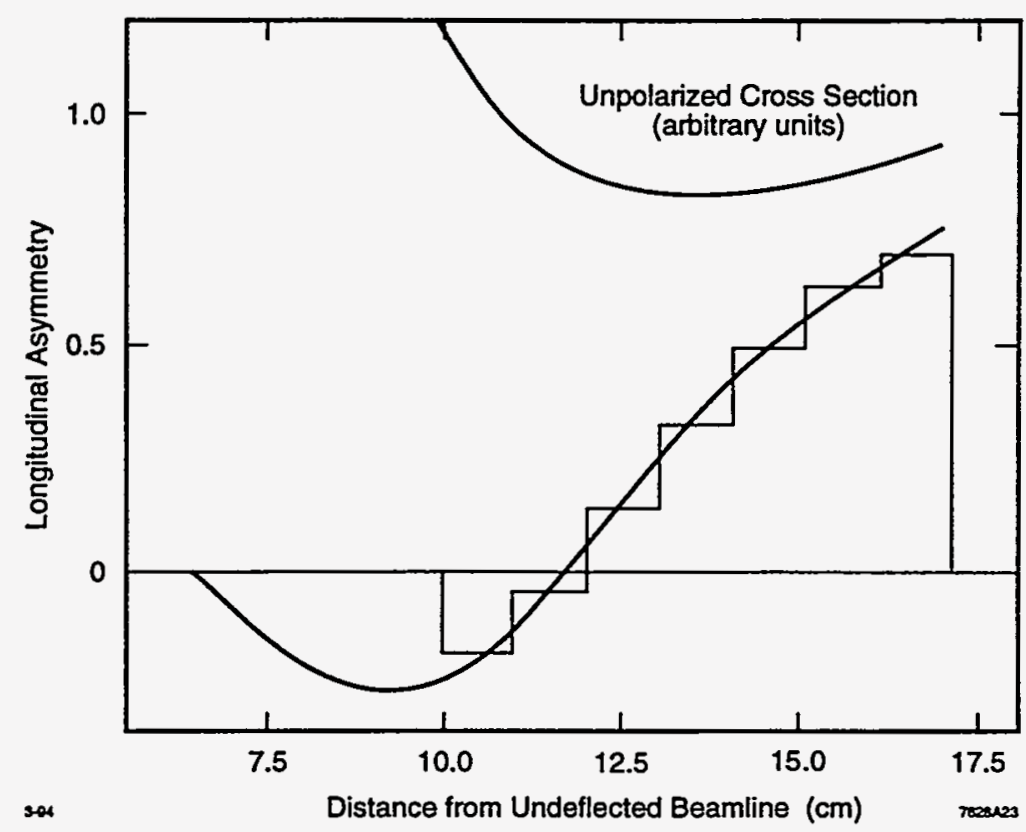

Figure 3.9: The Compton asymmetry and unpolarized cross section plotted vs. transverse distance from the undeflected beamline. The Cerenkov detector position and analyzing powers are shown in the histogram. All positions are final analysis results.

be small, as the analyzing powers closely track the asymmetry function.

\subsection{Polarization Determination}

As discussed in Chapter 1, the luminosity-weighted average electron beam polarization over the run is used to extract $A_{L R}$ from the measured $Z$ asymmetry. Determination of the beam polarization requires understanding the Čerenkov detector calibration, linearity, and electronic corrections, as well as the Compton light circular polarization [54].

\subsubsection{Detector calibration}

In order to interpret the asymmetry measured in each Cerenkov channel, we must know the position of the Compton electron spatial spectrum relative to the Cerenkov detector. The theoretical form of the asymmetry function, kinematics of the interaction, and geometry of the Cerenkov detector are all well understood. The remaining degrees of freedom are the product of the Compton dipole bend strength and $z$ distance of the detector from the bend point, fixing the width of the distribution 
at the detector, and the transverse position of the detector. These two quantities can be found by measurement of any two relative positions between the electron spectrum and the Čerenkov detector. We choose to measure: 1) the transverse distance at the $z$ position of the front of channel 5 between the Compton edge and the outer edge of the outer wall of channel 6; and 2) the transverse distance at the same $z$ position between the zero-asymmetry point and the center of channel 2. We find the first distance using an edge scan, measuring the cross section in channel 6 , while the second distance is found using the measured asymmetries. Use of these different techniques avoids common systematics to either technique in both measurements.

\section{Compton edge determination}

In this measurement, we take advantage of the sharp Compton edge to provide a high-resolution comparison between data and Monte Carlo. We have seen that the Compton edge lies inside channel 7. Hence, by translating the Čerenkov detector transversely outward from the beamline on its movable table, we can measure the decrease in total signal in channel 6 as less and less of the spectrum falls inside the channel, and compare this curve with the simulated prediction.

The simulation is performed by choosing a reasonable starting position for the detector and using the nominal dipole bend strength (as the edge scan is only very weakly affected by small bend-strength deviations for $\sim 2 \mathrm{~cm}$ motion), translating the detector $1 \mathrm{~mm}$ at a time away from the beamline, and finding the total simulated signal in channel 6 by integrating the resulting response function convoluted with the Compton cross section over the channel limits. The detector table is assumed to move transversely to the detector axis (center of channel 5). Since the table cannot rotate the projective cells during translation, the scan induces an angular misalignment of the Cerenkov detector of $2.81 \mathrm{mr} / \mathrm{cm}$, which is included in the Monte Carlo.

The edge scan was performed twice, both with and without $8.5 \mathrm{~mm}$ lead shielding in front of the detector. The channel 6 edge scan with lead provides not only a determination of the edge position, but also a test of the shape of the lead-in response functions. The channel 6 data with lead, after removal of residual beam backgrounds and normalization to the cross section-normalized channel 3 signal (to remove Compton luminosity fluctuations), is plotted in Fig. 3.10. The curve is the result of a cubic spline fit to the data. The fit of Monte Carlo to data is performed by assigning statistical errors to the Monte Carlo scan points and minimizing the $\chi^{2}$ deviation of the simulated points from the cubic spline fit. The Monte Carlo point error determination is made by calculating the integrated error in the response functions as the scan progresses. The spline fit uncertainty is nearly 
as large as the experimental uncertainty in each data point, as the $\chi^{2} /$ dof for the spline fit is 0.12 , implying that the spline curve follows the data without interpolation; the experimental point errors are comparable in size to the Monte Carlo errors for all but the last few points. The fit of simulated points with spline curve is shown in Fig. 3.11. The horizontal scale is obtained from simultaneous readback of the calibrated linear potentiometer on the detector table. The horizontal position of the simulated points has been allowed to float to provide the best fit (using the potentiometer calibration of $0.359 \mathrm{~V} / \mathrm{cm}$ ). The simulation signal scale and offset are also fit parameters. There is good agreement in shape between the data and simulation, most importantly in the tails in both directions. The fit result is that the Compton edge lies $8.70 \pm 0.03 \mathrm{~mm}$ outside the outer edge of the outer channel 6 wall at a $z$ position of $355.8 \mathrm{~cm}$, or $3.70 \pm 0.03 \mathrm{~mm}$ outside the center of channel 7 . However, the $\chi^{2} /$ dof for this fit is 1.9 ; hence, the systematic error in the simulation, which is not addressed in the fitting procedure, is significant. Allowing the Monte Carlo point errors to increase so that $\chi^{2} /$ dof approaches unity does not change the fit results but increases the errors, giving an edge position of $3.70 \pm 0.05 \mathrm{~mm}$ outside the center of channel 7 .

The no-lead edge scan provides a second measurement of the edge position. The data, corrected as in the previous case and fit with a cubic spline, is plotted in Fig. 3.12. The fit of Monte Carlo points with spline curve is shown in Fig. 3.13. Again, the simulation transverse position and vertical scale and offset are allowed to float to provide the best fit. Some systematic disagreement between simulation and data is seen in the slope at the left of the scan and in the fall-off shape in the center. The result of this scan is that the Compton edge lies $3.54 \pm 0.01 \mathrm{~mm}$ outside the center of channel 7 at $355.8 \mathrm{~cm}$ past the bend point. The $\chi^{2} /$ dof for this fit is 9.4 , due primarily to the presence of systematic Monte Carlo error. Again, increasing the simulation errors to give a $\chi^{2} /$ dof of one gives the same fit results but increases the edge position error, yielding $3.54 \pm 0.04 \mathrm{~mm}$. Thus the nolead and lead edge scans differ by $0.16 \mathrm{~mm}$, a $2.5-\sigma$ discrepancy, pointing most likely to systematic Monte Carlo error. The effect of small intrinsic rotational detector misalignment has been studied preliminarily and does not bring the simulated edge scans into agreement. In any case, the $0.16 \mathrm{~mm}$ discrepancy leads to a negligible difference in terms of calibration, to be discussed below. Preferring to err on the side of conservatism, we choose an error twice as big as the discrepancy and quote the transverse distance from the center of channel 7 to the Compton edge as $3.6 \pm 0.3 \mathrm{~mm}$. 


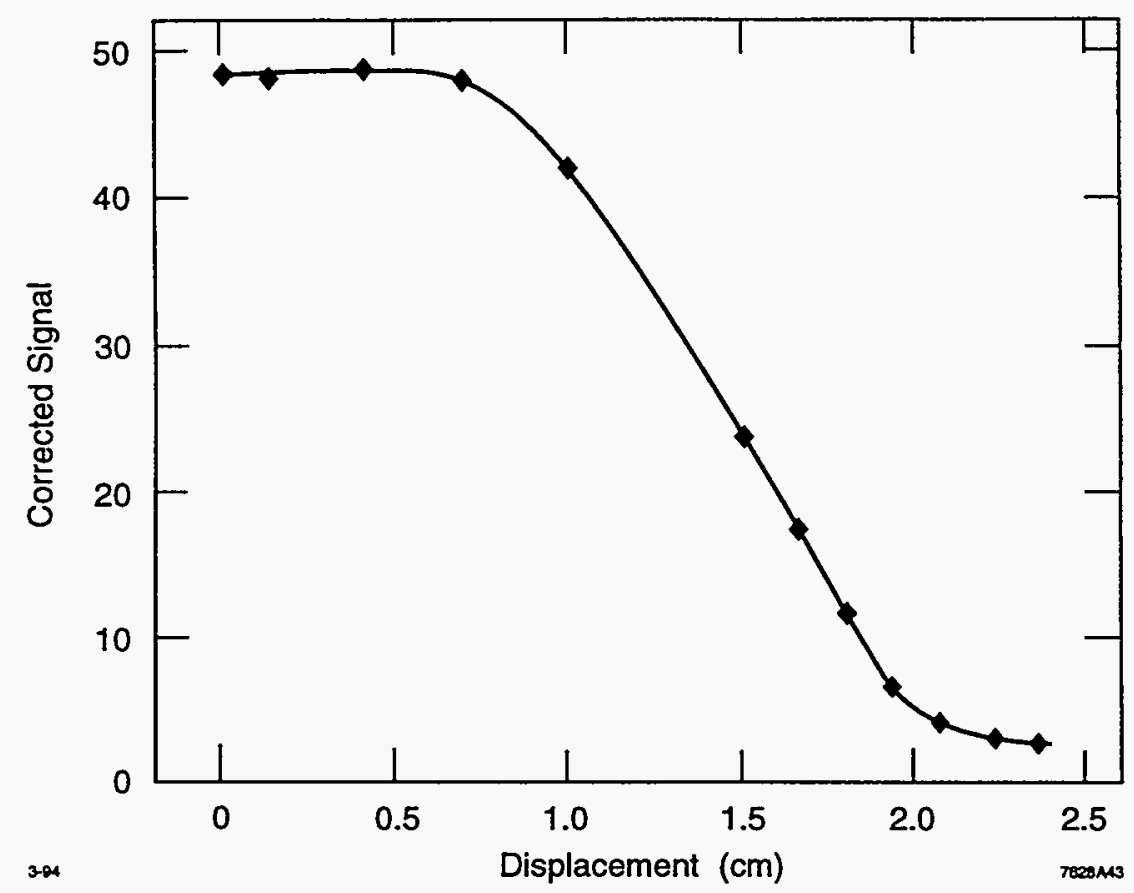

Figure 3.10: Cubic spline fit to channel 6 edge scan data with lead shielding in front of detector.

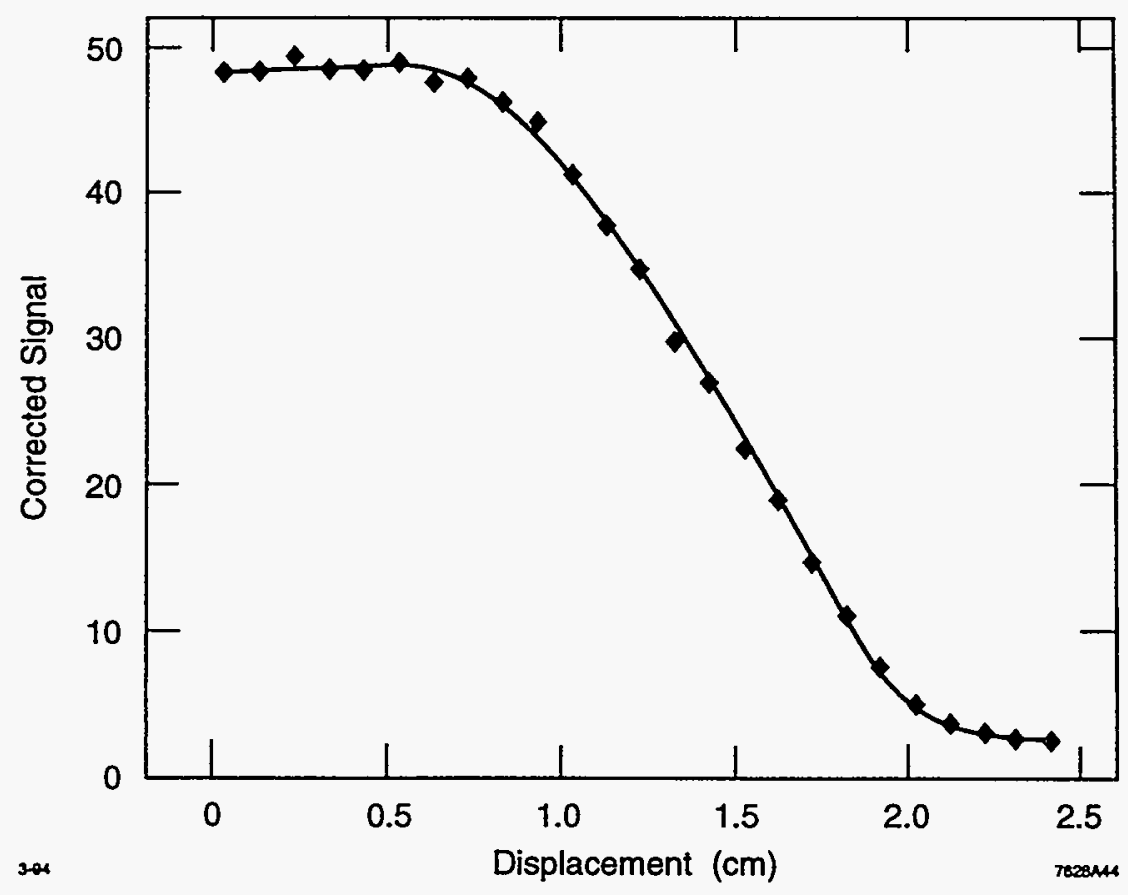

Figure 3.11: Fit of Monte Carlo to spline fit of data. 


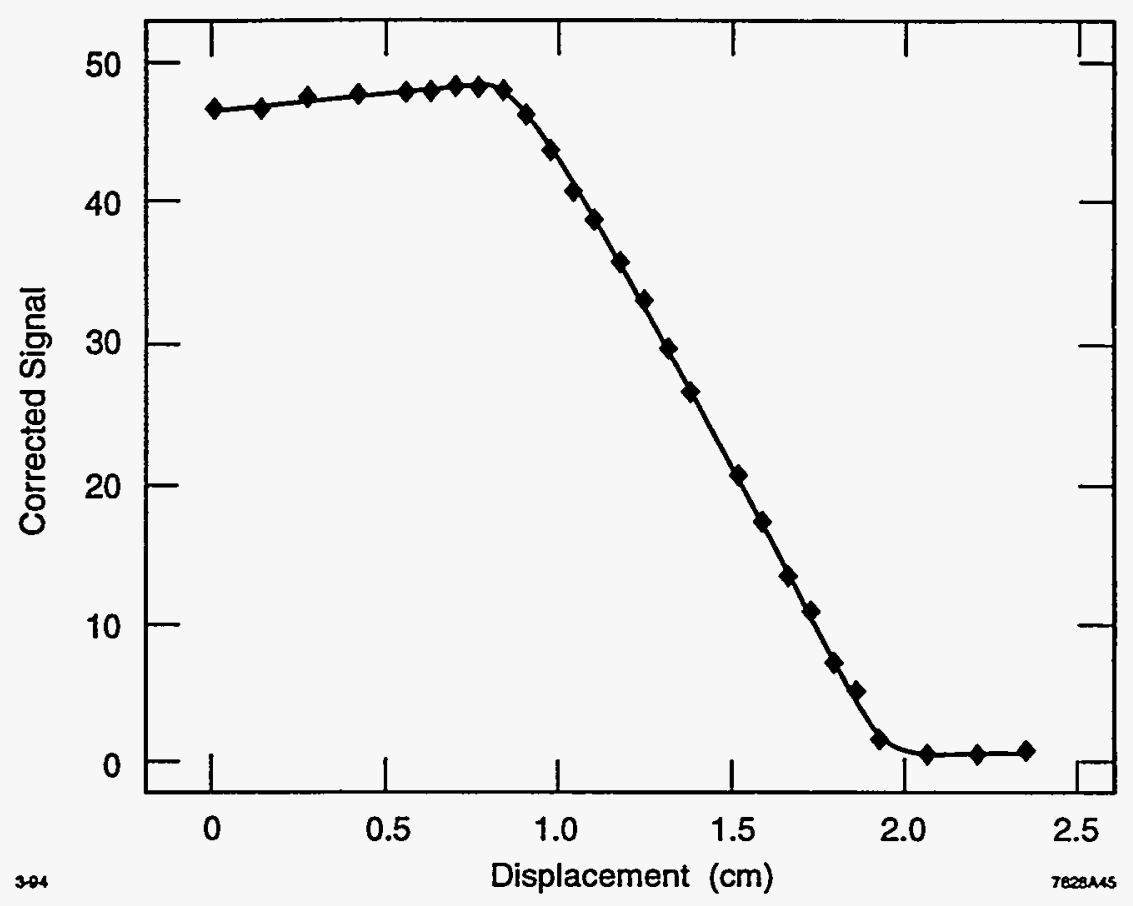

Figure 3.12: Cubic spline fit to channel 6 edge scan data with no lead shielding in front of detector.

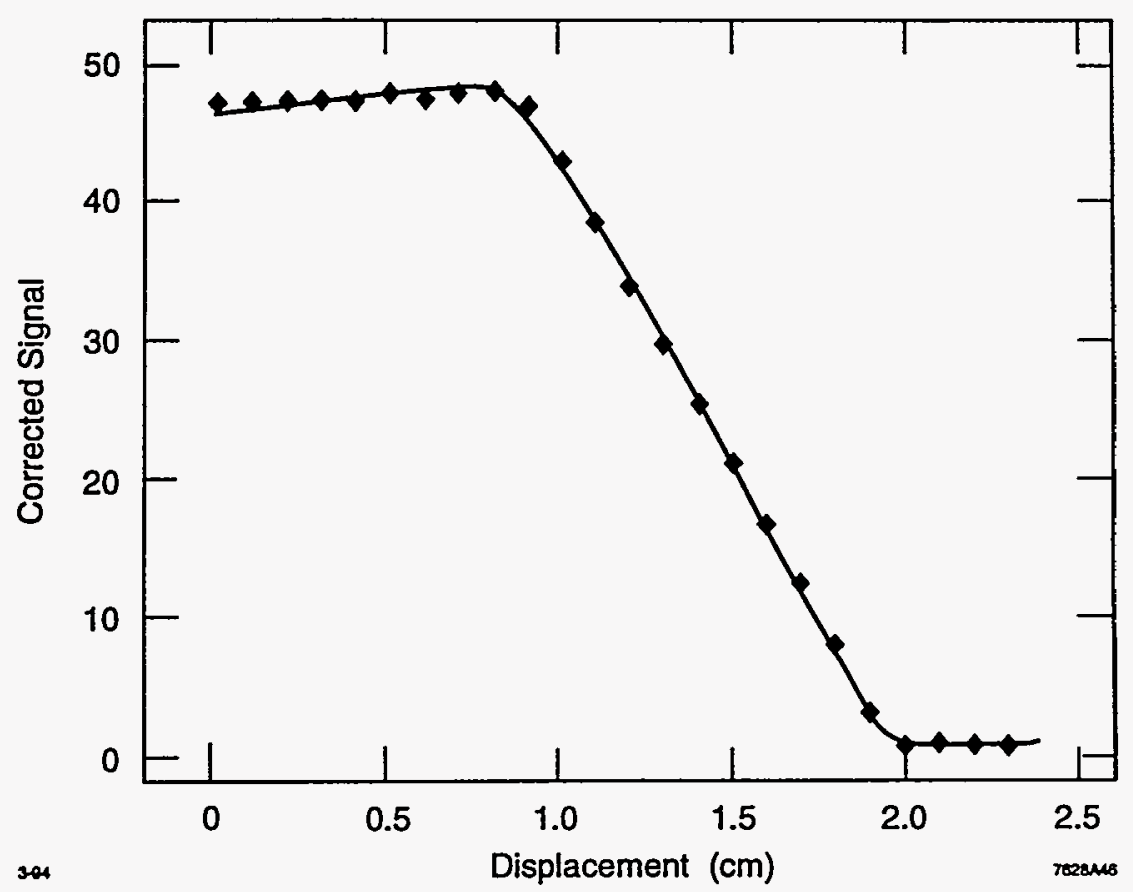

Figure 3.13: Fit of Monte Carlo to spline fit of data. 


\section{Zero-asymmetry-point determination}

The method of finding the zero-asymmetry point of the Compton spectrum is more straightforward. This point is observed to fall between the centers of channels 2 and 3; thus, taking advantage of the large accumulation of data on tape, we form the full-run average of the quantity

$$
A_{0} \equiv\left|\frac{A_{2}}{A_{3}-A_{2}}\right|
$$

where $A_{i}$ is the measured asymmetry in channel $i$. In the limit of ideal channel response, straight asymmetry function, and flat cross section, the value of $A_{0}$ defines the distance of the zero-crossing point from the center of channel 2 as a fraction of a channel width. We obtain $\left\langle A_{0}\right\rangle=0.22 \pm 0.01$. We then use the EGS simulation to find what zero-crossing position leads to this value for $A_{0}$, i.e., what zero-crossing position gives lead-shielded analyzing powers $a_{2}$ and $a_{3}$ for channels 2 and 3 such that

$$
\left|\frac{a_{2}}{a_{3}-a_{2}}\right|=0.22 \text {. }
$$

This procedure corrects for the nonidealities noted above. The result is that these effects shift the perceived zero-asymmetry point by $0.01 \mathrm{~cm}$ toward the beamline, so the zero-asymmetry point occurs $0.23 \pm 0.01 \mathrm{~cm}$ outside of the center of channel 2 .

\section{Calibration results and additional errors}

We can now use our Čerenkov detector calibration to obtain the channel analyzing powers and also to calculate the analyzing dipole bend strength and compare with the nominal value.

The nominal combined bend angle of the SB1 soft bend and the $\mathrm{B} 1$ hard bend magnets is $18.28 \mathrm{mr}$ for the on-energy beam of $M_{Z} / 2=45.58 \mathrm{GeV}$, giving a transverse momentum kick of $833.2 \mathrm{MeV}$ to electrons of all energies [55] From our measurements of the Compton edge and zero-crossing positions and our knowledge of the interaction kinematics and detector geometry, we can measure this combined bend strength. The position of the effective bend point has been calculated [55], and the distance from this point to the entrance of Cerenkov channel 5 has been measured to be $355.8 \pm 1.0 \mathrm{~cm}$. From the results in the last section, the transverse distance between the Compton edge and the zero-asymmetry point is

$$
5 \times 1.025+0.36-0.23 \pm 0.03 \mathrm{~cm}=5.26 \pm 0.03 \mathrm{~cm},
$$


giving an angle $\Theta$ of $14.78 \pm 0.08 \mathrm{mr}$ between the two rays. Thus the bend strength $B$ is given by

$$
\begin{aligned}
B & =\frac{\Theta}{\left[\frac{1}{E_{e d g e}}-\frac{1}{E_{0}}\right]} \\
& =\frac{(14.78 \pm 0.08) \times 10^{-3}}{\left[\frac{1}{17360 \mathrm{MeV}}-\frac{1}{25159 \mathrm{MeV}}\right]} \\
& =828 \pm 5 \mathrm{MeV}
\end{aligned}
$$

This bend strength is $0.6 \%$ from the nominal value and agrees within error. This independent measurement of $B$ using both measured asymmetries and cross sections provides a strong check on the Čerenkov detector calibration.

The calibration allows us to compute the analyzing powers $a_{i}$ of all the Cerenkov channels. The results were shown in Fig. 3.9 and are listed in Table 3.1, for the case of an ideal step response function and for $7.6 \mathrm{~mm}$ lead shielding. The lead shielding affects the analyzing powers of the high-asymmetry channels by less than $1.5 \%$.

\begin{tabular}{|c|c|c|}
\hline Cerenkov channel & $\boldsymbol{a}_{\boldsymbol{i}}$ ideal & $\boldsymbol{a}_{\boldsymbol{i}} \mathbf{7 . 6} \mathbf{~ m m}$ lead \\
\hline 1 & -0.1828 & -0.1794 \\
\hline 2 & -0.0387 & -0.0402 \\
\hline 3 & 0.1456 & 0.1422 \\
\hline 4 & 0.3306 & 0.3262 \\
\hline 5 & 0.4917 & 0.4877 \\
\hline 6 & 0.6205 & 0.6154 \\
\hline 7 & 0.7125 & 0.7027 \\
\hline
\end{tabular}

Table 3.1: Calculated analyzing powers for the Čerenkov detector. Column 2 uses a step-function response; column 3 uses EGS-derived response functions.

The Compton luminosity in the 1992 SLD run had large pulse-to-pulse fluctuations, due mainly to shifting local intensities in the laser spot at the Compton IP. These fluctuations cause large statistical uncertainties in the measured channel asymmetries, but these uncertainties are correlated among all channels. As explained in the previous chapter, the 1992 data acquisition system did not store pulse-by-pulse raw data, and hence the channel-to-channel correlations could not be measured. For this reason, fits to all channels simultaneously cannot be performed, as statistical errors cannot be properly evaluated, and we must use a single channel to calculate the electron polarization. We choose the highest-asymmetry channel, channel 7, except for the short period ( 1000 Zs ) at the beginning of the run when only channels 2 and 6 were active, in which case we use channel 6 . 
The uncertainties in the channel 6 and 7 analyzing powers depend on the errors in the measurements of $B$ and of the edge position. The uncertainty due to $\delta B$ is found by preserving the edge position relative to the center of the channel and calculating the analyzing powers using response functions generated with varying values of $B$. We find the $a_{i}$ errors corresponding to a $B$ uncertainty of $5 \mathrm{MeV}$ to be $\pm 0.16 \%$ in channel 6 and $\pm 0.04 \%$ in channel 7 . The uncertainty in $a_{i}$ due to the edge scan error is found by holding $B$ constant and varying the transverse channel position. The errors in $a_{i}$ corresponding to the $\pm 0.03 \mathrm{~cm}$ edge position uncertainty are $\pm 0.52 \%$ in channel 6 and $\pm 0.19 \%$ in channel 7. The analyzing powers of the outer channels are relatively insensitive to bend strength, assuming the edge position is well measured, as the distortion in the asymmetry function and cross section due to an incorrect $B$ will be small close to the edge. We see also that channel 7 is much less sensitive to either source of uncertainty than is channel 6 . The reason for the relative $B$ insensitivity is as stated above, since channel 7 is closer to the edge calibration point. The edge scan error has less effect on channel 7 than on channel 6 because the slope of the asymmetry function flattens out at the edge, implying less position sensitivity for channel 7 , and channel 7 is only partially filled with Compton signal, so the mean asymmetry in the channel changes only half as fast with channel translation as does that of channel 6 .

The statistical Monte Carlo uncertainty in the analyzing power determination is found by varying independently the random number seeds controlling the electron beam selection and showering in the detector. This error is found to be $0.07 \%$ per EGS run in the analyzing powers; each $a_{i}$ quoted in the table is the result of two EGS runs with different random number seeds, so this error becomes $0.05 \%$.

Cerenkov channel angular misalignment is a potential cause of error in the determination of analyzing powers. Simulation indicates that an intrinsic channel misalignment results in an edge scan with a shape that is indistinguishable from that of an aligned-channel edge scan; however, the transverse position is displaced according to the degree of misalignment. Such an edge scan is shown in Figs. 3.14 and 3.15. The simulated scan is performed in Monte Carlo channel 6 with an intrinsic $+5 \mathrm{mr}$ misalignment (positive misalignment angles correspond to counterclockwise channel rotations about the front of the channel as viewed from above), and with $8.5 \mathrm{~mm}$ lead shielding, as in the experimental edge scan. The top plot is a repeat of Fig. 3.11, the best fit of aligned simulated edge scan (crosses) to data (solid line), with the $+5 \mathrm{mr}$ simulated edge scan in circles, using the same position calibration as in the aligned simulation. The lower plot shows the $+5 \mathrm{mr}$ edge scan shifted to best agree with the data. The observed shift of $0.0596 \pm 0.0068 \mathrm{~cm}$ arises from the transverse 
motion of the channel centroid under rotation about the front of the channel; one expects $\sim 0.05 \mathrm{~cm}$ for $5 \mathrm{mr}$ of rotation given the $10 \mathrm{~cm}$ half-length of the channel. No information can be obtained regarding misalignment from the shape of the experimental edge scan; in order to estimate the effect of misalignment on the analyzing powers, we must misalign and shift the simulated channel by the above $0.0596 \pm 0.0068 \mathrm{~cm} / 5 \mathrm{mr}$ and compare the resulting analyzing power with that of an unshifted, aligned channel. The results of this study are shown in Figs. 3.16 and 3.17. The Monte Carlo statistical uncertainty is seen in the error bars on the central $0 \mathrm{mr}$ points, while the increasing error bars toward the ends manifest the angle-dependent channel shift uncertainty. Conservatively taking the maximum slope allowed by the error bars, we find an uncertainty in the analyzing powers of $0.08 \%$ per milliradian of misalignment in channel 6 and $0.03 \% / \mathrm{mr}$ in channel 7 . As mentioned earlier, the detector was surveyed before and after the run and found to be within a few milliradians of proper alignment; therefore, the misalignment error is negligible.

\section{Time dependence of calibration}

The position calibration of the Čerenkov detector can change over time due to electron beam position or energy shifts or to potential fluctuations in the dipole bend strength. We must monitor the calibration and include variations in our error.

Changes in the endpoint position are most sensitively observed in the total signal in channel 7 , as the Compton edge falls within the channel. To eliminate Compton luminosity fluctuations, we normalize the background-subtracted channel 7 signal to that of channel 2, whose total signal is much less sensitive to experimental variations affecting the calibration, and plot the result in Fig. 3.18 vs. polarimeter run number. Each bin represents an average over 600 polarimeter runs. In addition, a requirement is made on each polarimeter run that the number of signal pulses plus background pulses be over 200 , and that all four helicity-combination bins have at least one entry. The large excursions are all understood: the spike at run $\sim 30,000$ is due to a phototube linearity sweep, to be described below; no data is available at run 22,000; and the broad peak between runs 15,000 and 25,000 is due to changing phototube gains during periods of increasing Compton luminosity. The largest remaining excursion is $\sim 5 \%$ from the value of the ratio when the edge scan was performed at run $\sim 12,500$, and the average deviation is half this big, corresponding to a channel 7 position shift of $0.03 \mathrm{~cm}$, equal to the edge position uncertainty. We make no correction for this shift but include it as an additional systematic error in our analyzing power determination.

The quantity $A_{0}$, defined in Eq. 3.13, is plotted as a function of polarimeter run, again averaged 


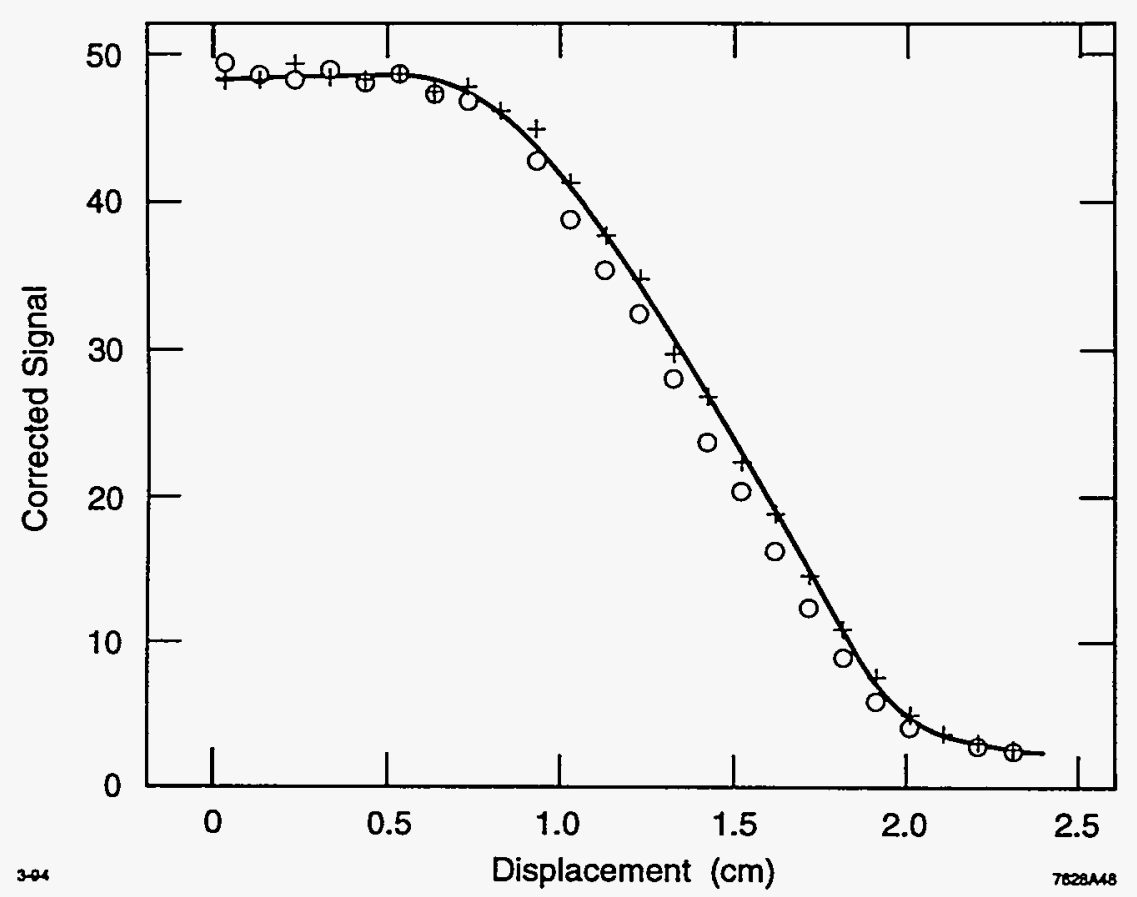

Figure 3.14: The $+5 \mathrm{mr}$ simulated $8.5 \mathrm{~mm}$ lead channel 6 edge scan (circles) is placed on the same horizontal scale with the best fit of aligned simulated edge scan (crosses) to data fit (solid line). The uncorrected relative transverse position of the simulated scans is shown.

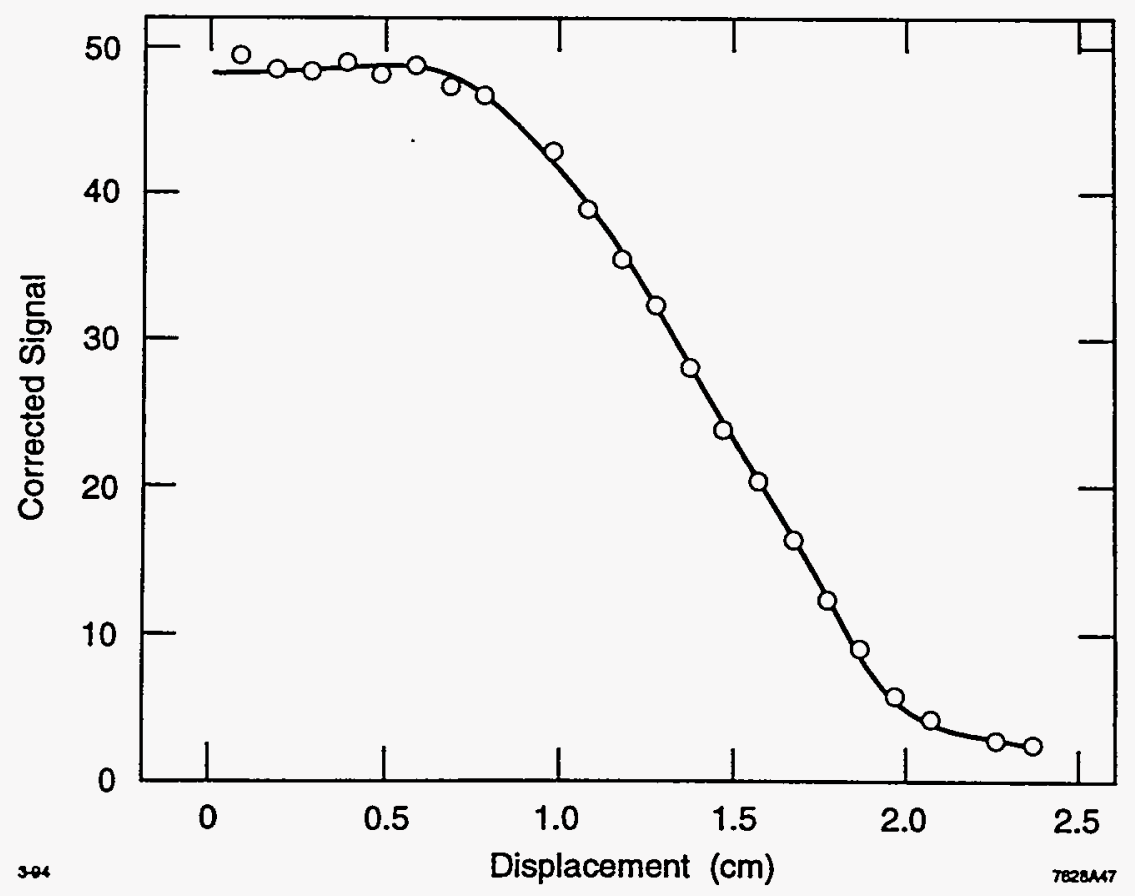

Figure 3.15: The $+5 \mathrm{mr}$ edge scan (circles) shifted to provide the best fit with data fit (solid line). 


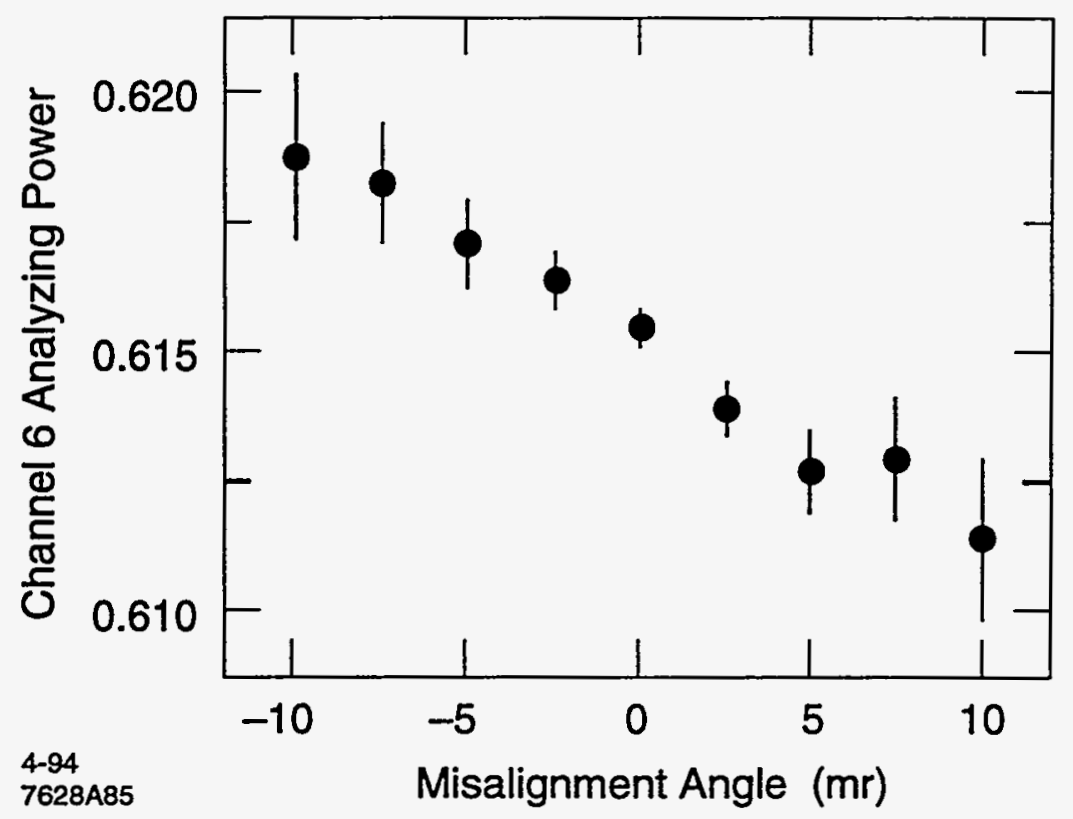

Figure 3.16: Plot of channel 6 analyzing power as a function of channel misalignment angle. Error bars include Monte Carlo statistical uncertainty in analyzing power determination as well as uncertainty in transverse shift with misalignment angle.

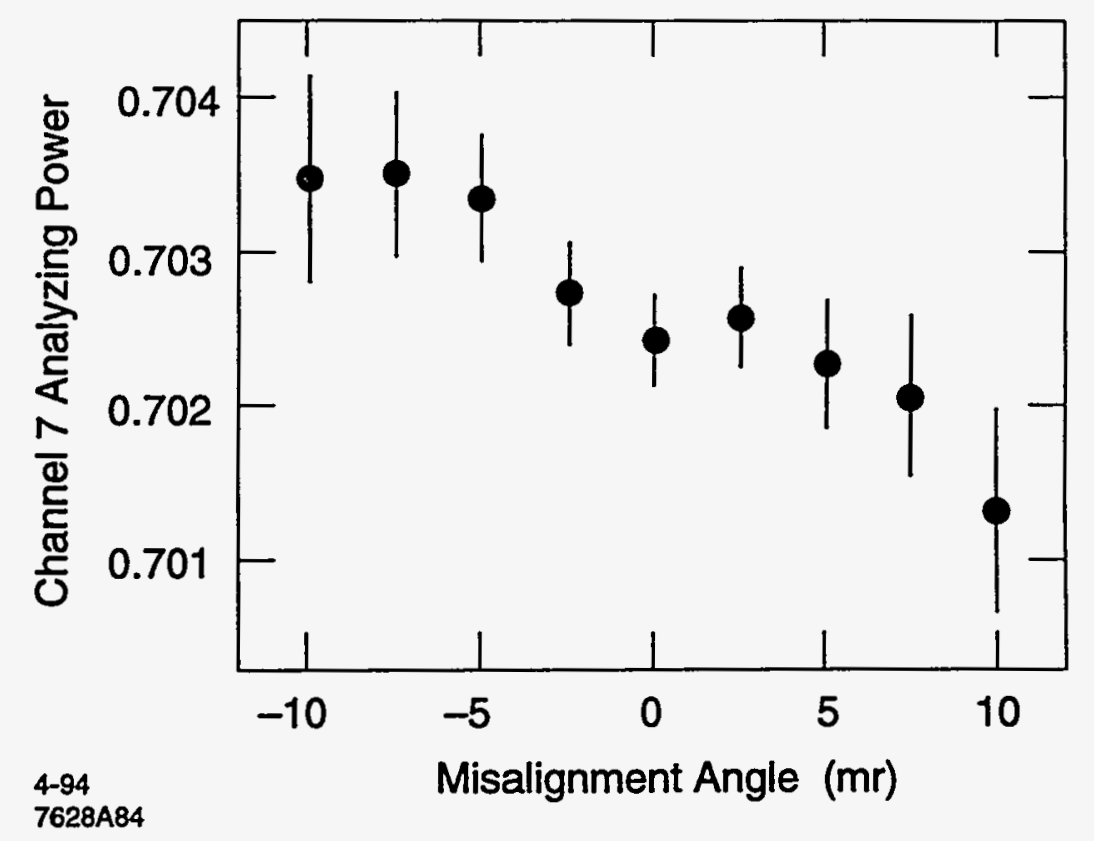

Figure 3.17: Channel 7 analyzing power vs. misalignment angle. 


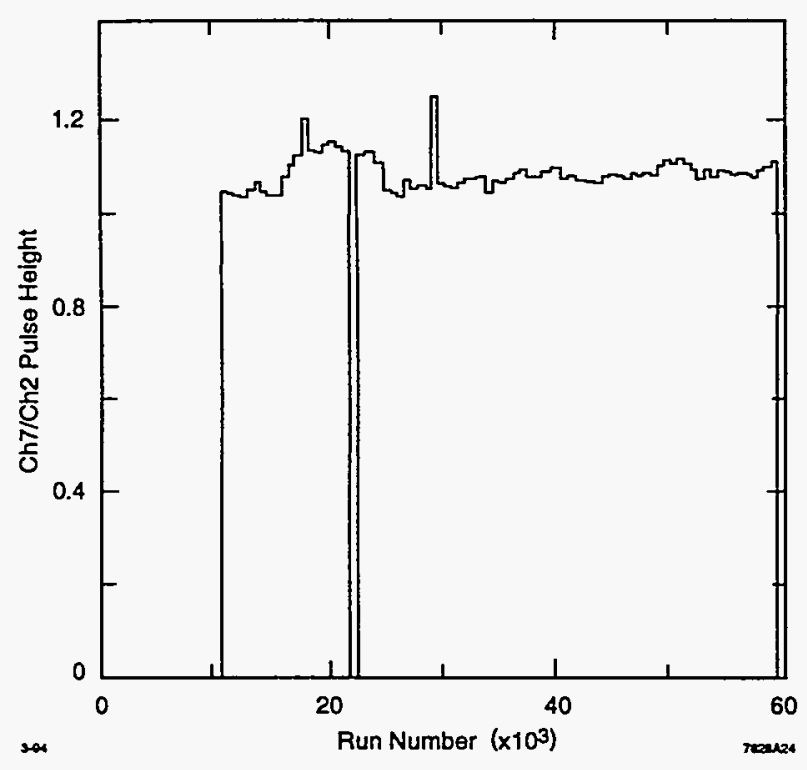

Figure 3.18: Time history of the ratio of background-subtracted pulse heights in channels 7 and 2 plotted vs. polarimeter run number.

in groups of 600 and using the same cuts on runs, in Fig. 3.19. This quantity is a monitor of the zeroasymmetry point and provides another check on experimental fluctuations. As $A_{0}$ was defined and presented as an average over the entire run, its value and error need not be amended. In addition, the absence of any statistically significant deviations obviates a time-dependent value of $A_{0}$ for use in determining the run-averaged beam polarization. In any case, as noted earlier, the channel 6 and 7 analyzing powers are only weakly dependent on the zero-asymmetry position.

\subsection{2 Čerenkov detector linearity}

As detailed in the previous chapter, phototube linearity in the Cerenkov detector was an early concern in the measurement of the beam polarization. Nonlinearity can occur through space-charge saturation above some supply-voltage-dependent signal level, which was the major motivation for the phototube/base development effort, or through a distortion in the response curve at signal levels below the space-charge limit. Either of these effects will cause a mismeasurement of the Compton asymmetry if the nonlinearity occurs at the experimental signal height. In addition, other detector effects, such as electronics nonlinearities and background biases, can contribute to shifts in the measured asymmetry for different phototube pulse heights. All these concerns can be addressed with the detector in situ, using polarized beam data. 


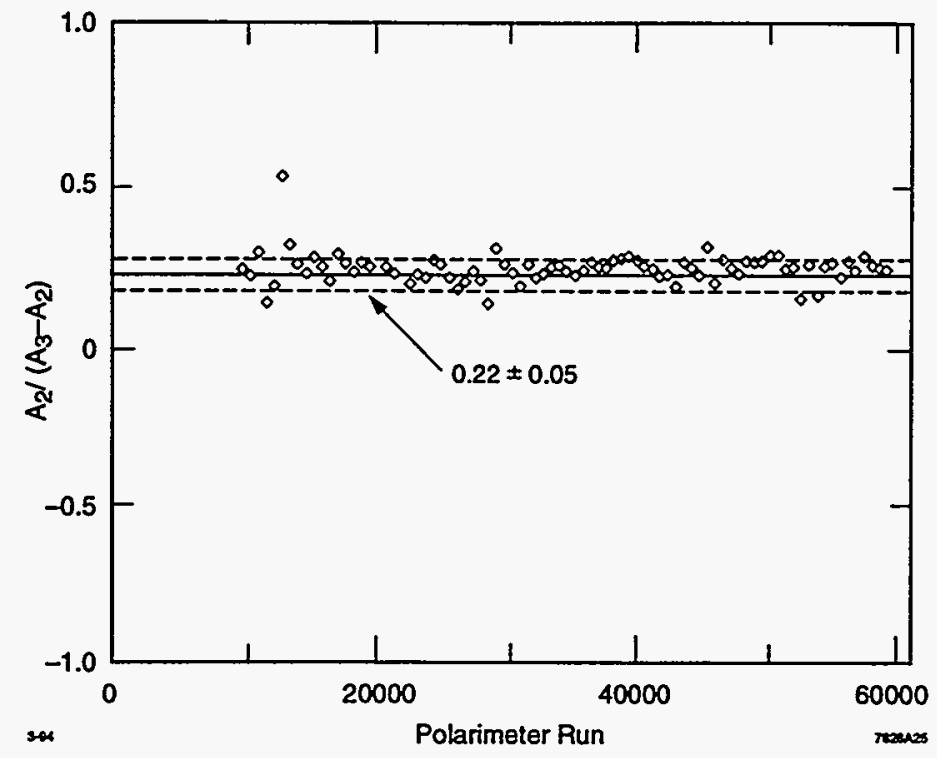

Figure 3.19: $A_{0}$ plotted as a function of polarimeter run number.

\section{Saturation check}

The space-charge saturation point of the phototubes was checked twice in short tests by holding the back-end tube voltage $\epsilon_{2}$ fixed at the normal operating voltage of $600 \mathrm{~V}$, fixing the spacecharge current limit, while increasing the front-end voltage $\epsilon_{1}$ to observe the effect of saturation in the channel 6 and 7 measured asymmetries. In order to reduce scatter and measure true detector effects, the beam polarization must be monitored and included. To do this, we use simultaneous data from the PTD. In the 1992 run, the PTD had a known nonlinearity in response, causing a mismeasurement of the asymmetries. Fig. 3.20 shows the ratio of the polarization measured in the PTD to that in the Čerenkov detector, for Cerenkov signals well below saturation levels, plotted vs. PTD signal-plus-background pulse height averaged over seven PTD channels. Here we are not concerned with details of the Čerenkov nonlinearity, only with gross saturation effects; hence, we assume that the Cerenkov response is approximately linear in the data used for this graph. The results of channel 6 and 7 voltage saturation scans, normalizing the measured channel asymmetry by the corrected measured PTD polarization, are shown in Figs. 3.21 and 3.22. The data was acquired with the nominal value of $\epsilon_{2}=600 \mathrm{~V}$. We see the onset of saturation occur in both channels at $\sim 300$ ADC counts, corresponding to a pulse height of $\sim 1.0 \mathrm{~V}$, consistent with the behavior observed in the LBL bench tests (see Fig. 2.16). Typical pulse heights during the run were 80-150 counts. 


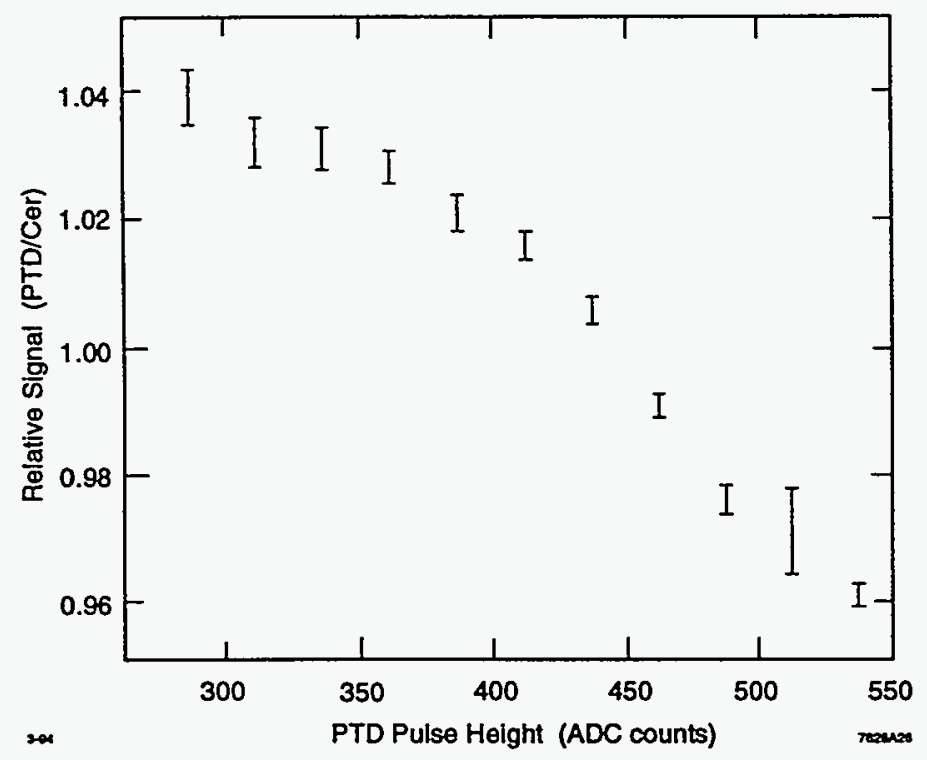

Figure 3.20: The ratio of polarization measured in PTD to that in Čerenkov detector plotted vs. PTD signal-plus-background pulse height averaged over seven PTD channels.

\section{Linearity measurement}

To address the question of detailed detector/readout linearity in the pulse-height range of experimental interest, we turn to the data on tape from the polarized-beam running after tube voltages were held constant. We now divide the Čerenkov channel 6 and 7 data into twelve bins according to average pulse height during the polarimeter run and histogram the observed channel asymmetry in each bin. Again, we must normalize by the true beam polarization for each run, which we measure using the calibrated PTD. In this case, however, we cannot use the Čerenkov detector data to calibrate the PTD, as this procedure would remove the details of the Čerenkov nonlinearities under study. Here we choose to divide the PTD data into four bins based on pulse height, adjusting the Cerenkov response separately for each PTD bin, and rely on the general lack of correlation between the pulse heights of the two detectors to wash out the PTD nonlinearities within each bin. Due to the uncorrelated pulse heights, each PTD bin contains polarimeter runs in which the Cerenkov response covers nearly the entire pulse-height range. Thus, the Cerenkov linearity study could be done with any of the PTD bins; however, we wish to combine the bins to take advantage of the increased statistics. The relative normalization of the Cerenkov response curves in the four PTD bins must be done at the same Cerenkov pulse height, which is allowed due to the overlapping of the Čerenkov curves. In addition, Čerenkov linearity information at low pulse heights depends 


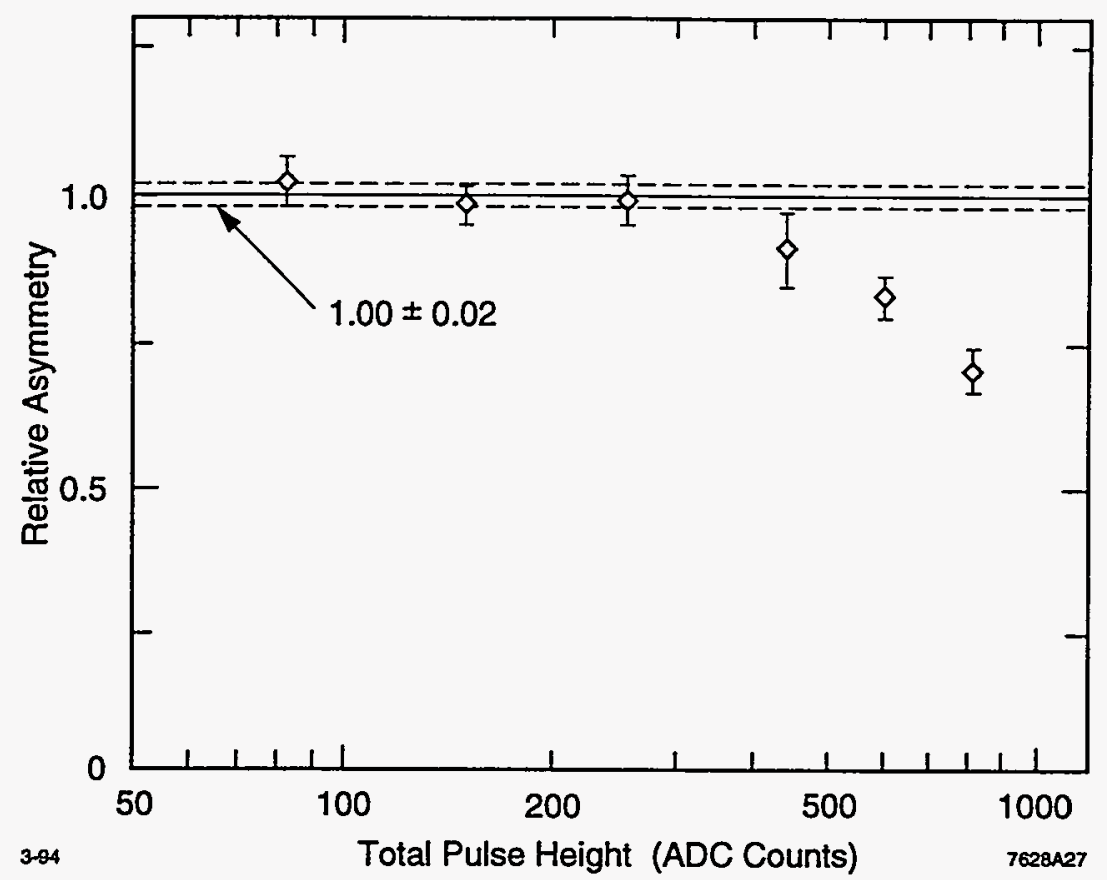

Figure 3.21: Measured Compton asymmetry in Čerenkov channel 6 relative to corrected measured PTD polarization, plotted vs. channel 6 pulse height.

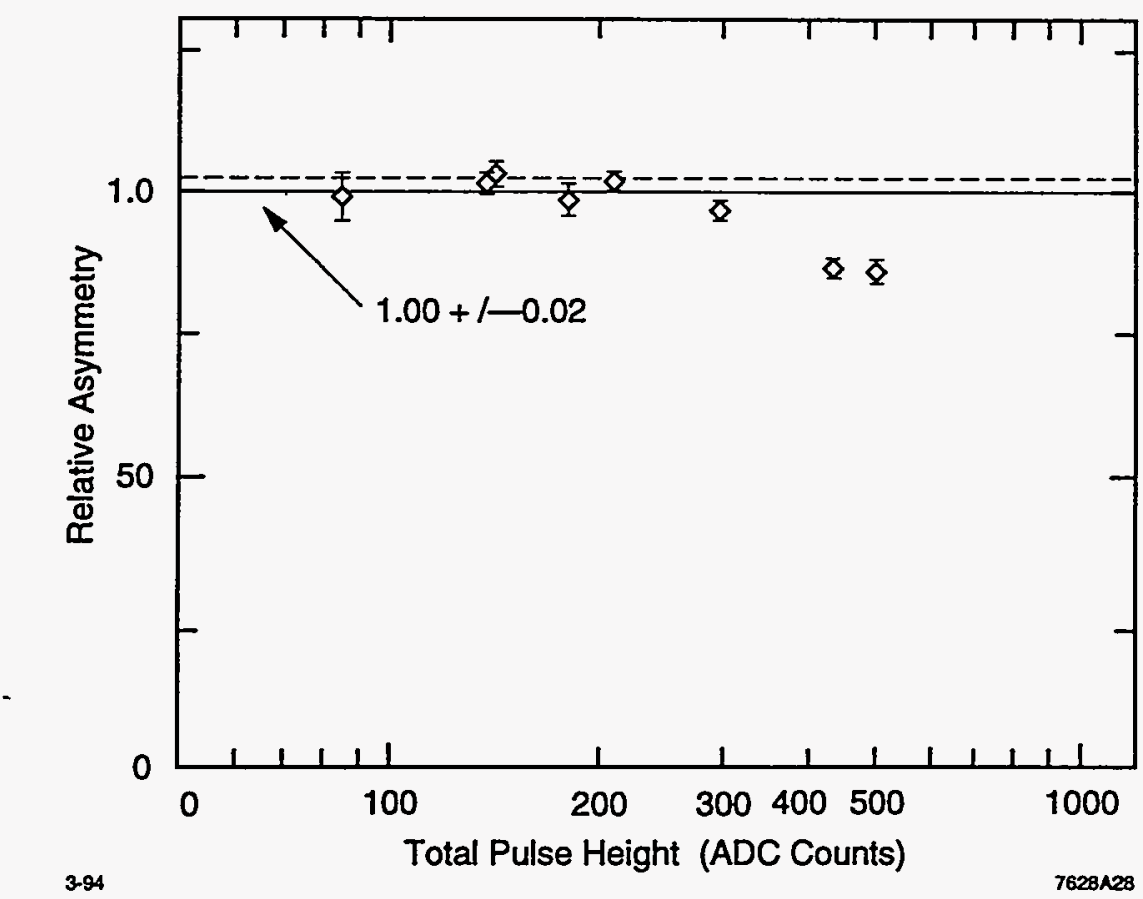

Figure 3.22: Measured Compton asymmetry in Čerenkov channel 7 relative to corrected measured PTD polarization, plotted vs. channel 7 pulse height. 
on proper accounting of electronic noise and cross-talk in the ADCs, to be discussed below. The results of these studies for Črenkov channels 6 and 7 are shown in Figs. 3.23 and 3.24. It can be shown that the fractional asymmetry mismeasurement at a particular pulse height is the difference between the value of the linearity curve at that pulse height and the $y$-intercept of the tangent to the linearity curve at that pulse height. Using the information in Figs. 3.23 and 3.24, we find that the total relative asymmetry error for the data sample is less than $1.5 \%$. The flat central pulse height region of 50 to 150 counts includes approximately $93 \%$ of the data. Outside this region, we have parametrized the observed nonlinearities and evaluated the size of the asymmetry measurement error, assigning systematic error on the order of the size of the effects. We also cut any polarization run with an average of less than 35 counts of total pulse height (signal plus background), or an average of less than 5 counts of signal. Since very little data was taken outside the linear region, the effect of these cuts and corrections is almost unobservable. Our error estimate of $1.5 \%$ is consistent with predictions of linearity curves obtained in LBL bench tests (cf. Figs. 2.16 and 2.17) and from independent tests performed at Hamamatsu on the phototubes used in the Čerenkov detector.

We note that for most of the run ( $80 \%$ of the integrated luminosity), beam crossings were vetoed from the data acquisition if the laser-off background level measured in Čerenkov channel 9 exceeded 200 counts. Channel 9 lies over $1 \mathrm{~cm}$ beyond the Compton edge and receives almost no signal; hence it is not used to measure the beam polarization. This cut ensured that backgrounds in channels 6 and 7 remained below 100 counts and protected against large variations in the backgrounds due to beam scans and bad pulses. An examination of the limited $120 \mathrm{~Hz}$ data available on tape in the ring buffers indicates that such background fluctuations had a small effect on the measured asymmetries ( $<0.5 \%$ relative) for the running before the channel 9 veto was installed.

\subsubsection{Electronic noise and cross-talk}

Electronic noise pickup in the Čerenkov detector ADCs introduces a bias in the asymmetry measurement. Noise of 0.5-1.0 ADC counts from the laser Q-switch signal is observed when the laser fires and hence is not seen as background and subtracted from the laser-on Čerenkov pulses. We have measured this pickup as a function of time using the available $120 \mathrm{~Hz}$ data on tape for which no electron beam was present. We ascribe an uncertainty of 0.3 counts to the measurement, leading to an extra systematic uncertainty of $0.3 / S_{i}$, where $S_{i}$ is the signal size in channel $i$. For typical signals of $\sim 100$ counts, this is a $0.3 \%$ effect. 


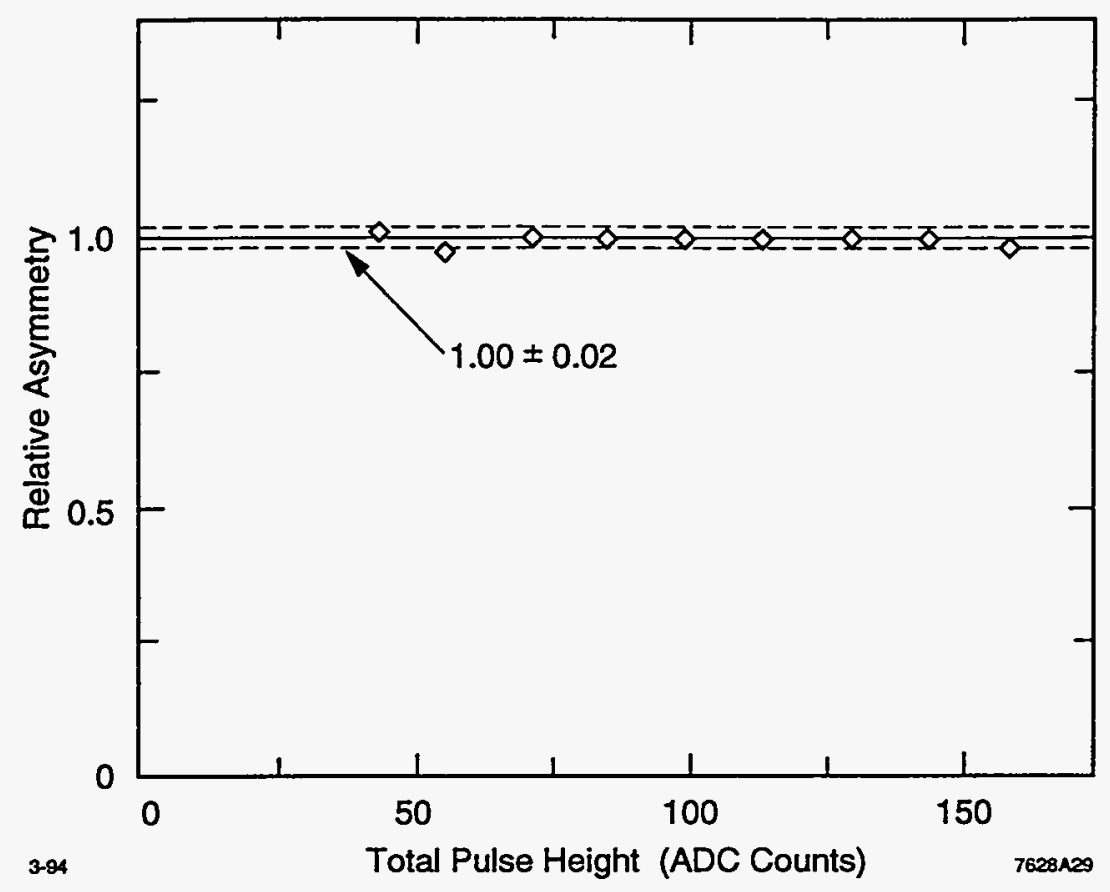

Figure 3.23: Relative asymmetry measured in Cerenkov channel 6 in the nominal pulse-height range.

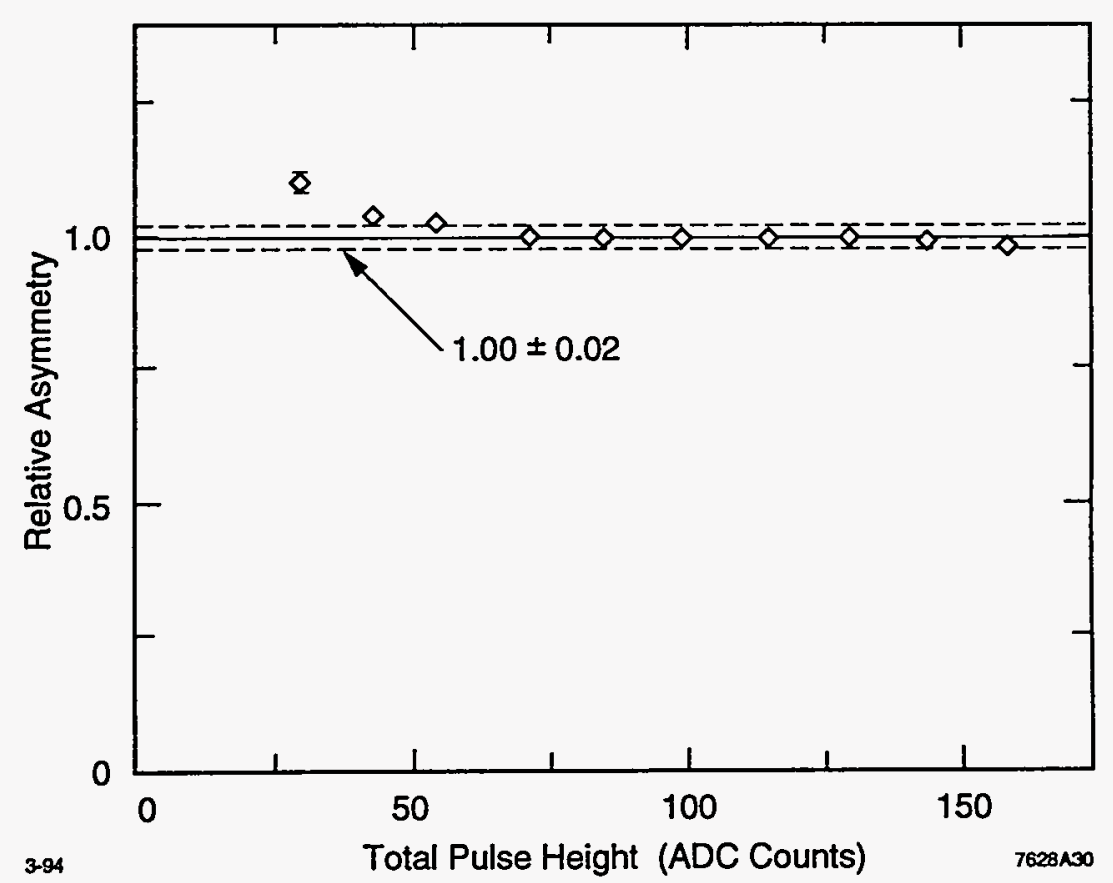

Figure 3.24: Relative asymmetry measured in Cerenkov channel 7 in the nominal pulse-height range. 
Using the LED pulsers mounted in front of the phototubes, we have measured the channel-tochannel cross-talk, including phototube bases, cables, and the ADCs themselves, to be $\sim 0.3$ counts per volt of signal, or about $0.1 \%$. The level of cross-talk scales with pulse height and hence is negligible for all running conditions.

\subsubsection{Light polarization measurement}

The Compton photon circular polarization measurement is of crucial importance to the electron polarization determination, as the measured Compton asymmetry is a product of $\mathcal{P}^{e}, \mathcal{P}^{\gamma}$, and $a_{i}$ (Eq. 3.10). We discuss here the method and results of this measurement [56].

\section{Experimental procedure}

Fig. 3.25 shows the experimental setup in measuring the laser circular polarization. A rotatable

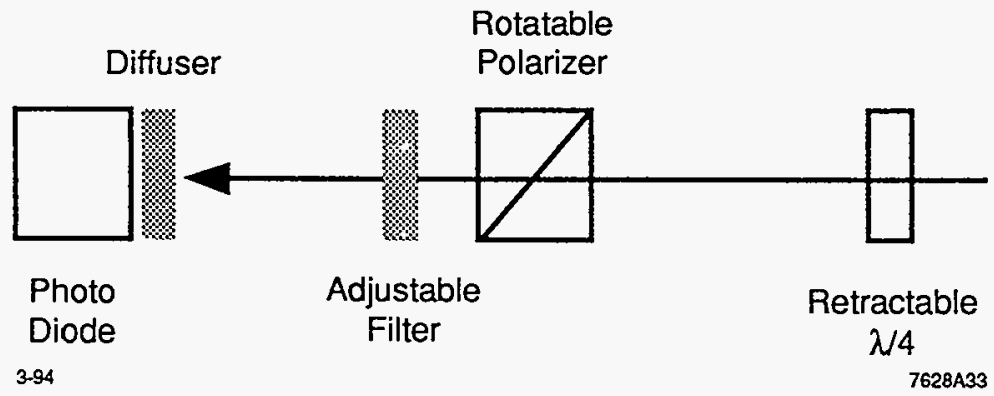

Figure 3.25: Schematic of experimental setup for measuring light circular polarization and handedness.

linear polarizer is inserted into the circularly polarized beam to pick out the length of the electric field vector along the linear polarizer axis. As the polarizer is rotated, the positions corresponding to the maximum and minimum intensities measured in the photodiode indicate the major and minor axes of the light polarization ellipse, and the degree of circular polarization is, in the absence of unpolarized light,

$$
\mathcal{P}^{\gamma}=\frac{2 \sqrt{R}}{1+R}
$$

where $R$ is the ratio of intensities measured in these two positions. The retractable quarter-wave plate is used to determine the absolute beam helicity, a topic to be discussed in the final chapter. As discussed in Chapter 2, the circular polarization is continuously monitored on the laser bench and in 
the analysis box; however, we require knowledge of $\mathcal{P}^{\gamma}$ at the Compton IP. The direct measurement of $\mathcal{P}^{\gamma}$ at the IP was made twice, once before the SLD run and once after, by breaking the SLC vacuum and using the above apparatus. In both cases, $\mathcal{P}^{\gamma}$ was also measured just after the Pockels cell on the laser bench, and before the run it was measured in two other places along the optical transport line: on the laser bench just before the vacuum transport line (VTL) window, and in the tunnel just after the VTL exit window. The pre-run measurements were made using a joulemeter read out directly on an oscilloscope, while the post-run measurements used a photodiode read with a LeCroy 2249 ADC. We note that different Pockels cell samples of the same type were used for the two sets of measurements. For cell \#5, positive high voltage corresponds to negative-helicity light, while for cell \#20, positive high voltage corresponds to positive-helicity light [57]. All measurements are listed in Table 3.2. Each entry represents a number of independent measurements at the specified location.

\begin{tabular}{|l|c|c|}
\hline \multicolumn{1}{|c|}{ Measurement location } & Date & $\mathcal{P}^{\gamma}$ \\
\hline After PC \#5, +1850 V (4 msmts.) & $1 / 4-5 / 92$ & $0.991 \pm 0.004$ \\
\hline Before VTL entrance window (2 msmts.) & $1 / 5 / 92$ & $0.986 \pm 0.006$ \\
\hline After VTL exit window (2 msmts.) & $1 / 5 / 92$ & $0.964 \pm 0.008$ \\
\hline At CIP, steering lens removed (10 msmts.) & $1 / 7 / 92$ & $0.933 \pm 0.004$ \\
\hline At CIP, steering lens installed (4 msmts.) & $1 / 7 / 92$ & $0.931 \pm 0.006$ \\
\hline \hline After PC \#20, +1800 V, -1700 V (4 msmts.) & $9 / 4 / 92$ & $0.994 \pm 0.002$ \\
\hline At CIP, +1800 V, -1700 V (4 msmts.) & $9 / 3 / 92$ & $0.930 \pm 0.010$ \\
\hline
\end{tabular}

Table 3.2: A summary of the invasive measurements of $\mathcal{P}^{\gamma}$ made along the laser beam line.

\section{Results}

We first summarize the general results of the measurements. The circular polarization on the laser bench, both before and after the back-reflecting window, is seen to be $\sim 99 \%$. Following exit from the VTL, the polarization has dropped to $\sim 96 \%$, while at the Compton IP, it falls to $\sim 93 \%$. The optical transport line for the laser light is shown in Fig. 3.26. We see that the first $\sim 3 \%$ loss is due to the two windows and six mirrors traversed in the VTL, from both birefringence in the optics and misalignment of the compensating mirrors. The second drop of $\sim 3 \%$ at the Compton IP must be attributed to misalignment or birefringence in the two mirrors in mirror mount \#4, or birefringence in the SLC entrance window. As mentioned in Chapter 2, the birefringence of this window was measured with the SLC both at atmospheric pressure and evacuated, and was found to be negligible. 


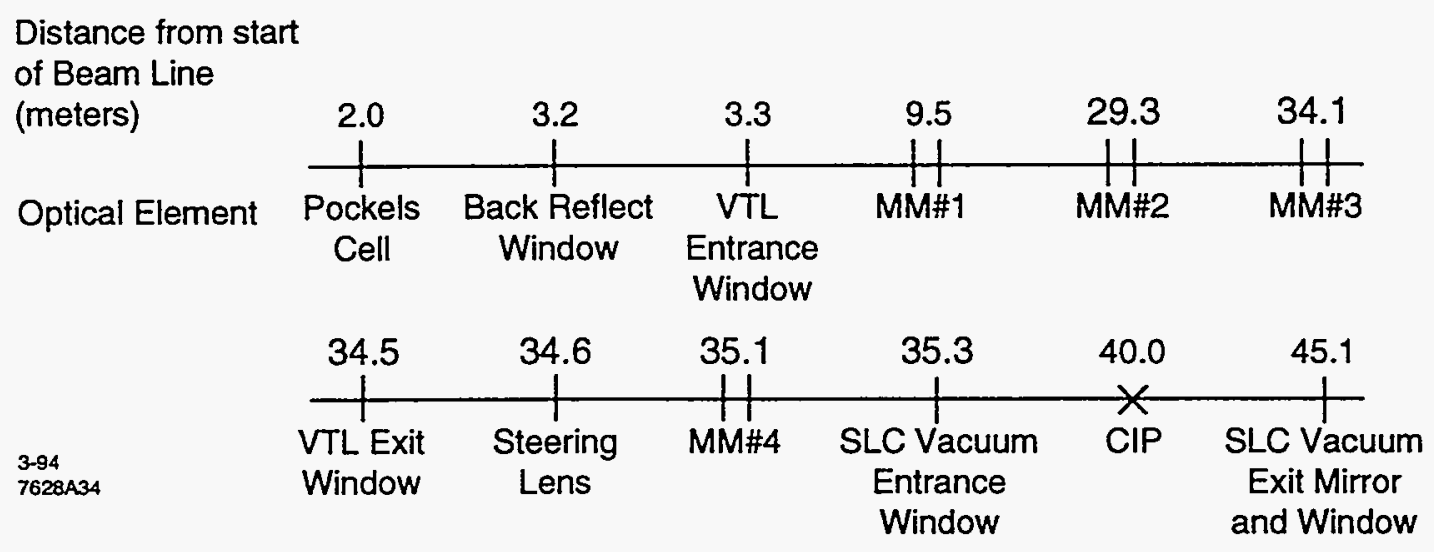

Figure 3.26: Schematic of the laser optical transport line.

The quoted measurement errors at each location are determined simply by considering the spread in values within each set of independent measurements. Thus, the uncertainties include only the effects of random experimental error, due mostly to the arbitrariness in determining the linear polarizer orientation giving maximum and minimum transmission. Other systematic errors could cause a deviation of the measured $\mathcal{P}^{\gamma}$ from its true value. Unpolarized light in the laser beam would mimic circular light, as its transmission through the linear polarizer would be independent of polarizer orientation. The unpolarized light component in the beam was not measured at the Compton IP, so we must allow for its possible presence. Preliminary results from 1993 running, in which the fraction of unpolarized light was measured and monitored, suggest its presence at the $<0.5 \%$ level [58]. An imperfect or systematically misaligned linear polarizer would also contribute to systematic error, but the magnitude of these effects was not or could not be measured.

In addition to the statistical uncertainty in the $\mathcal{P}^{\gamma}$ measurements, the time dependence of the circular polarization must be considered. Fluctuations in $\mathcal{P}^{\gamma}$ are monitored both on the laser bench and in the analysis box; however, for the reasons discussed in Chapter 2, the laser bench system is considered a more sensitive and accurate relative measure of $\mathcal{P}^{\gamma}$. The time history of the circular polarization measured on the laser bench is shown in Fig. 3.27. We choose not to correct the measured values of $\mathcal{P}^{\gamma}$ at the Compton IP using this data; rather, we include this $1 \%$ spread in the overall uncertainty in $\mathcal{P}^{\gamma}$.

Based on these arguments, we estimate the circular polarization of the laser light at the Compton IP to be

$$
\mathcal{P}^{\gamma}=93 \% \pm 2 \%
$$




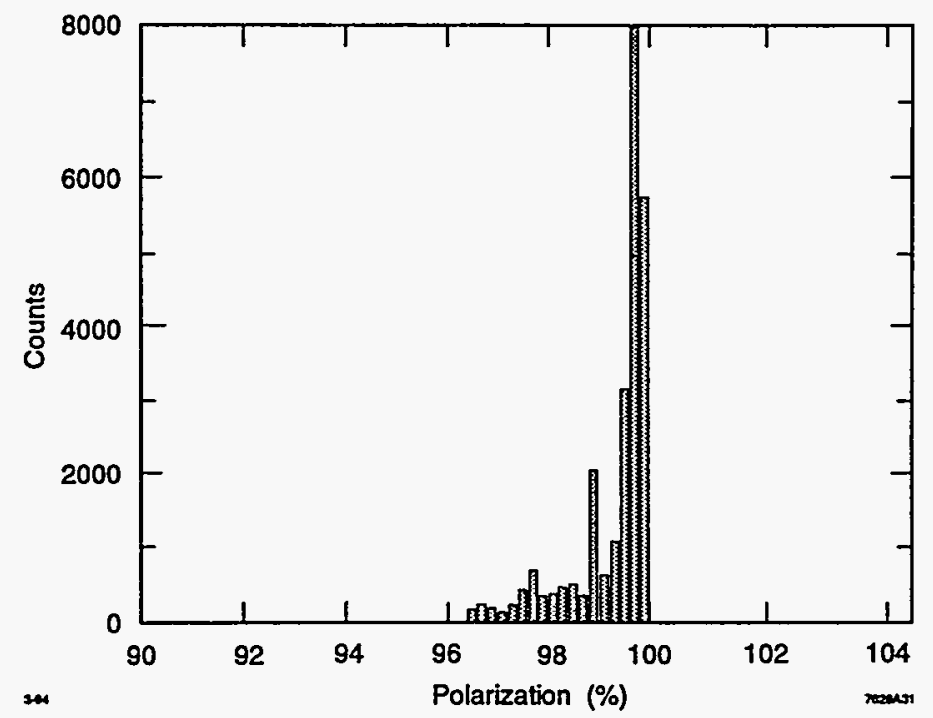

Figure 3.27: Circular light polarization measured on the laser bench throughout the 1992 run.

This error estimate is based on a conservative combination of the pre- and post-run measurement errors, time dependence, and healthy paranoia.

\subsubsection{Compton asymmetry spectrum}

As mentioned earlier in the chapter, the electron polarization measurement can be made with any of the Cerenkov channels, provided the detector position is calibrated. This channel-by-channel calculation provides a strong consistency check on our $\mathcal{P}^{e}$ measurement technique and reveals hidden systematic effects.

We assess discrepancies among channels by comparing a global average of $\left\langle A_{i}\right\rangle /\left\langle A_{7}\right\rangle$ for each channel $i$ with the expected ratios from the analyzing powers. To determine this experimental ratio in each Cerenkov channel, we make the following requirements on polarimeter runs:

- At least one beam crossing in each laser-on and background bin;

- At least 200 beam crossings measured, including background pulses;

- At least $2.54 \mathrm{~mm}$ lead inserted;

- Detector table within $300 \mu \mathrm{m}$ of nominal position;

- Statistical error in channel asymmetry measurement less than 0.015 ; 
- Total pulse height, including background, between 90 and 150 counts for channel $i$ and channel 7.

Table 3.3 shows in column 1 the $7.6 \mathrm{~mm}$-lead analyzing powers from Table 3.1 and in column 2 the measured global channel asymmetries. Column 3 lists the analyzing powers relative to the channel 7 value, and column 4 contains the measured asymmetries relative to that in channel 7 . The

\begin{tabular}{|c|c|c|c|c|}
\hline Cerenkov channel & $\boldsymbol{a}_{\boldsymbol{i}}$ & $\boldsymbol{A}_{\boldsymbol{i}}$ & $\boldsymbol{a}_{\boldsymbol{i}} / \boldsymbol{a}_{\boldsymbol{7}}$ & $\boldsymbol{A}_{\boldsymbol{i}} / \boldsymbol{A}_{\boldsymbol{7}}$ \\
\hline 1 & -0.1794 & $-0.0375 \pm 2 \mathrm{e}-4$ & -0.2552 & $-0.2577 \pm 0.0014$ \\
\hline 2 & -0.0402 & $-0.0082 \pm 7 \mathrm{e}-5$ & -0.0572 & $-0.0566 \pm 0.0005$ \\
\hline 3 & 0.1422 & $0.0275 \pm 3 \mathrm{e}-5$ & 0.2023 & $0.1889 \pm 0.0004$ \\
\hline 4 & 0.3262 & $0.0681 \pm 3 \mathrm{e}-5$ & 0.4642 & $0.4677 \pm 0.0003$ \\
\hline 5 & 0.4877 & $0.1020 \pm 3 \mathrm{e}-5$ & 0.6940 & $0.7011 \pm 0.0004$ \\
\hline 6 & 0.6154 & $0.1285 \pm 3 \mathrm{e}-5$ & 0.8758 & $0.8833 \pm 0.0003$ \\
\hline 7 & 0.7027 & $0.1455 \pm 3 \mathrm{e}-5$ & 1.0000 & 1.0000 \\
\hline
\end{tabular}

Table 3.3: Calculated analyzing powers $\left(a_{i}\right)$ and average measured raw asymmetries $\left(A_{i}\right)$ for each Čerenkov channel with $7.6 \mathrm{~mm}$ lead.

difference between columns 3 and 4 is a measure of the channel-to-channel polarization consistency. The largest difference, in channel 3 , is 0.013 . The difference of 0.0075 in channel 6 corresponds to a difference of $\Delta \mathcal{P}^{e} / \mathcal{P}^{e}=0.0086$ in measured polarization between channels 6 and 7 . This discrepancy is not explained by nonlinearity, electronic noise and cross-talk, or any other understood effect. Therefore, we include this difference as an additional systematic error. Fig. 3.28 shows the average raw asymmetry from column 2 plotted along with the predicted Compton asymmetry function vs. transverse position. The transverse position and horizontal scale of the asymmetry function is fixed by the Čerenkov detector calibration, while the vertical scale of the function has been adjusted to provide the best fit with the data, yielding $\mathcal{P}^{e}=22.4 \%$ at $\mathcal{P}^{\gamma}=93 \%$. The horizontal coordinates of the data are chosen so that the point for channel $i$ plotted at that position and with height equal to $a_{i}$ would fall on the asymmetry curve. Therefore, deviations of data from the curve reflect true channel deviations from the mean polarization measured by the detector. These deviations are shown in the inset to the figure.

The existence of some potential systematic effects can be ruled out by examination of the plot and residuals. An error in the detector calibration, in either position or bend strength, would show itself as a bowed vertical shift in the data with a maximum shift where the asymmetry function is steepest, at the zero-asymmetry point. This shift could not be removed by the fit, as the vertical scale is the only free parameter. A left-right luminosity asymmetry also would result in a vertical offset in the data. Finally, negative-asymmetry shower products, presumably due to small-angle 


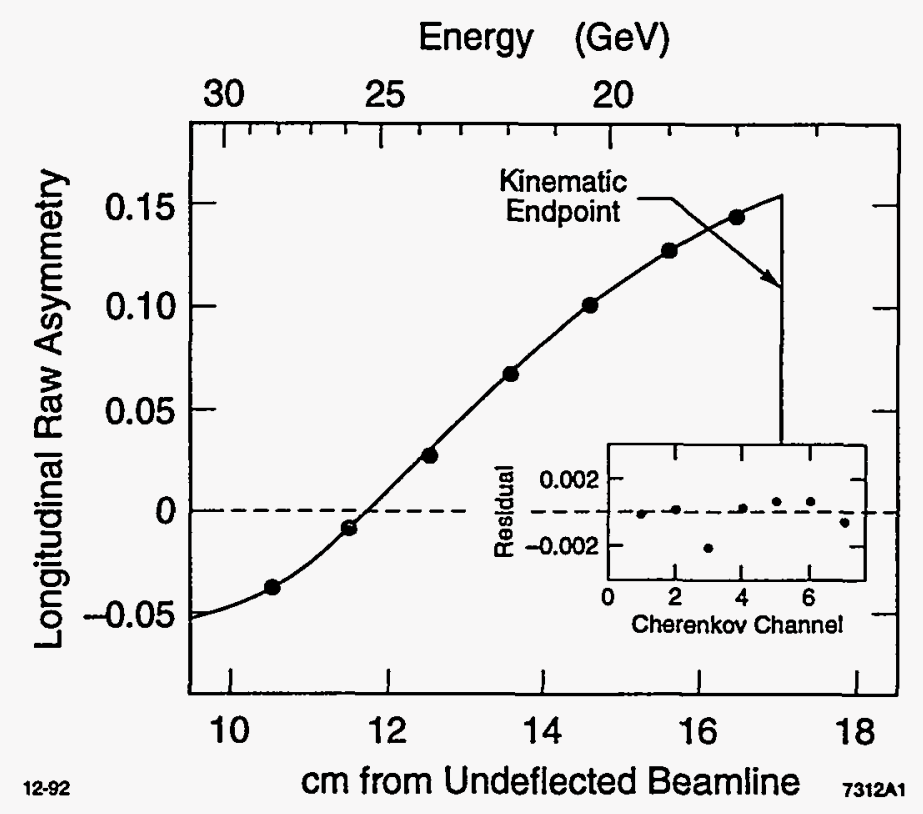

Figure 3.28: Comparison of observed Čerenkov channel asymmetries, averaged over all polarizedbeam running, with the theoretical Compton asymmetry function. Transverse scale and position of the asymmetry function is fixed by calibration, while vertical scale is chosen to give best fit with data, yielding $\mathcal{P}^{e}=22.4 \%$ at $\mathcal{P}^{\gamma}=93 \%$. Data residuals are shown in inset.

Compton electrons showering in the beampipe and SLC vacuum exit window, would lower the measured asymmetry by an increasing amount toward the inner channels. This phenomenon was in fact observed and studied during running without lead. None of these systematic effects is evident in the measured Compton spectrum at an experimentally significant level.

\subsubsection{Luminosity-weighted polarization results}

To determine the global luminosity-weighted longitudinal electron polarization for the 1992 run, we associate with each recorded $Z$ the beam polarization measured closest in time to the $Z$. For this measurement, we use channel 7 , except for the early period ( 1000 $Z$ s ) when channel 7 was not working, in which case we use channel 6 . We require the following of polarimeter runs:

- Total pulse height, including background, of the channel used must be greater than 35 counts;

- At least five counts above background in the relevant channel;

- At least five beam crossings in each laser-on and background bin;

- Uncertainty of less than 0.05 in the asymmetry measurement of the channel; 
- Phototube back-end voltage $\epsilon_{2}$ read out;

- Known lead configuration;

- Laser bench joulemeter asymmetry less than 0.5;

- Polarization of run less than $32 \%$.

In addition, the following corrections are applied to polarimeter runs:

- Nonlinearities are corrected by pulse height and channel as described earlier this chapter;

- Runs with nonstandard lead thicknesses (i.e., differing from $7.6 \mathrm{~mm}$ ) are corrected according to the simulated change in analyzing power with the corresponding lead thickness.

These corrections result in a $0.03 \%$ shift in the mean polarization.

Using the polarized $Z$ event sample described in the next chapter, we plot in Fig. 3.29 the polarization measured closest in time to each $Z$ event $v s$. event number. We see evidence of longterm structure in the polarization, in particular near the end of the run, when $\mathcal{P}^{e}$ remained above $25 \%$ for extended periods. These trends appear to be uncorrelated with any known machine or source effects that change the magnitude of the polarization, and are attributed to only partially understood accelerator parameters that cause a rotation of the polarization vector in the north arc. Maintaining the highest recorded polarization of $\sim 26 \%$ over the run would have resulted in the equivalent of a $35 \%$ luminosity improvement for the measurement of $A_{L R}$. Also shown in Fig. 3.29 is the same data in histogram form. Using Eq. 1.38, we find that the luminosity-weighted average longitudinal electron beam polarization for the run is

$$
\overline{\mathcal{P}^{e}}=22.37 \% \pm 0.02 \% \text { (stat.) } \pm 0.60 \% \text { (syst.) }
$$

The systematic error contributions discussed above are summarized in Table 3.4. The contribution shown is the average of the individual contributions from channels 6 and 7 , weighted according to the number of $Z \mathrm{~s}$ for which each channel was used to measure the beam polarization. We note 

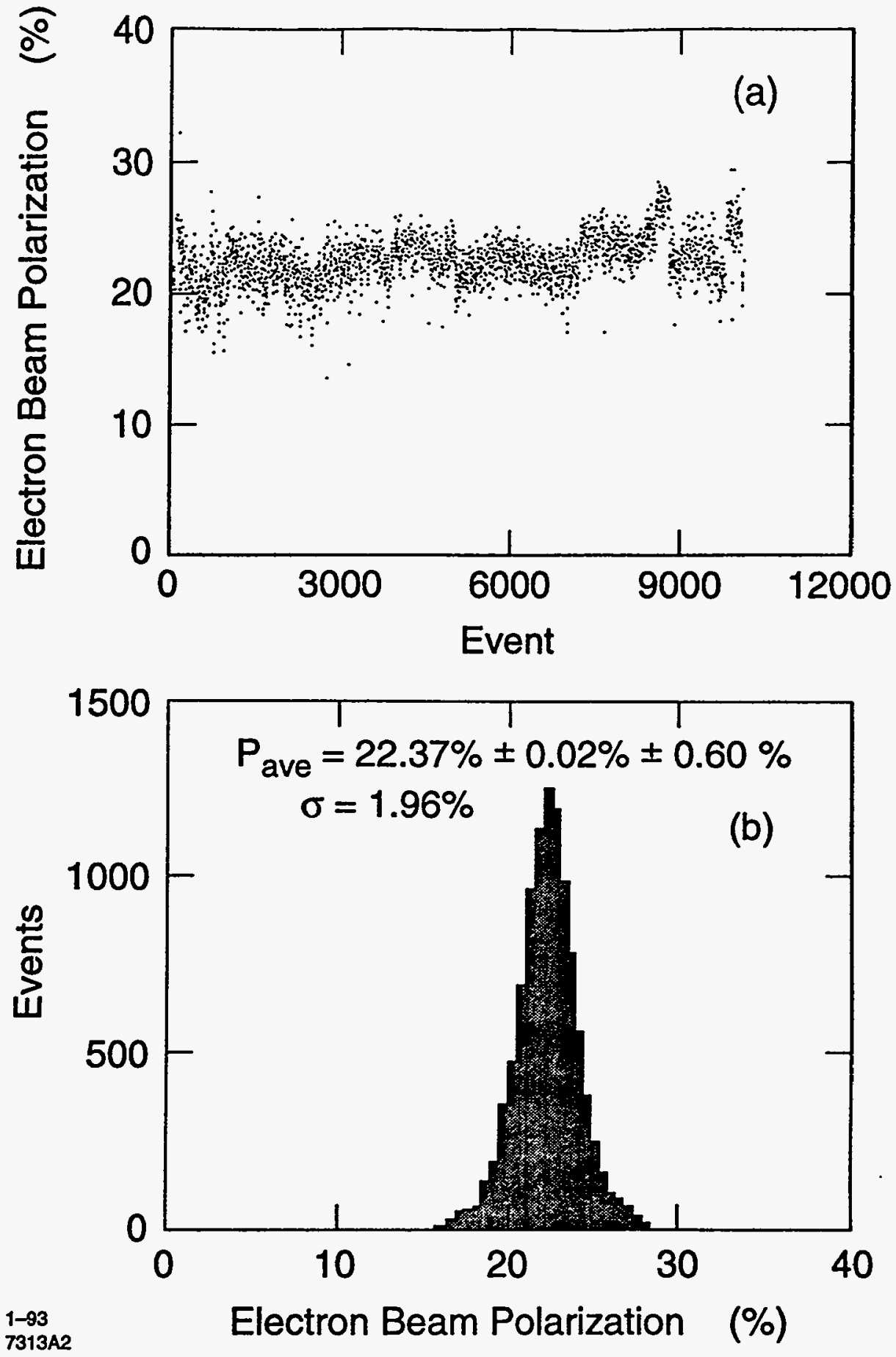

Figure 3.29: (a) Time history of electron polarization associated in time with each $Z$ event. (b) Distribution of electron polarization associated in time with each $Z$ event. 


\begin{tabular}{|c|c|}
\hline Systematic uncertainty & $\delta \mathcal{P} / \mathcal{P}$ \\
\hline Laser polarization & $2.0 \%$ \\
Detector linearity & $1.5 \%$ \\
Interchannel consistency & $0.9 \%$ \\
Spectrometer calibration & $0.4 \%$ \\
Electronic noise correction & $0.4 \%$ \\
\hline Total polarization uncertainty & $\mathbf{2 . 7 \%}$ \\
\hline
\end{tabular}

Table 3.4: Contributions to systematic error in measurement of luminosity-weighted average beam polarization.

that the $1.96 \%$ (absolute) width of the distribution is not explained by the average statistical error in each run of $\delta \mathcal{P}^{s t a t} \sim 0.8 \%$; thus, the width is due to real fluctuations in the longitudinal beam polarization during the run. 


\section{Chapter 4}

\section{Event Selection and Background}

\section{Estimation}

The measurement of $A_{L R}$ requires a $Z$ event sample as large as possible and undistinguished in any way except by its purity and by the absence of $e^{+} e^{-}$final states. Further, $A_{L R}$ is insensitive to any bias in the background except a left/right systematic difference, which is unlikely to occur; therefore, we are required only to estimate accurately the size of the contamination, contributing to an overall dilution of $A_{L R}$. Due to the simplicity of the required event selection, it is possible to make the selection using only calorimetry, a desirable option due to the immature status of the tracking analysis for the 1992 run and the relative quality of the SLD calorimeter. This chapter describes the "official" event selection procedure [59] using the LAC and the endcap WIC pads, as well as the method used to estimate the background component [60].

\subsection{Event selection}

The $Z$ sample is chosen in three stages from the triggered events on tape. The first stage loosely selects $Z$ candidates, including hadronic decays, tau pairs, and wide-angle $e^{+} e^{-} \rightarrow e^{+} e^{-}$, or Bhabha, events. As Bhabha events can proceed via left-right-symmetric photon exchange in the $t$-channel, we remove them with the second filter stage. Finally, beam backgrounds are tackled in stage 3 . The combined trigger and selection efficiency for this filter to find hadronic and tau-pair $Z$ events is estimated to be $92 \pm 2 \%$ [61]. 


\subsubsection{Trigger}

In the 1992 run, three different triggers were used for hadronic $Z$ decays:

- ENERGY: total liquid argon calorimeter (LAC) energy greater than $8 \mathrm{GeV}$;

- TRACK: at least two central drift chamber tracks separated by more than $20^{\circ}$;

- HADRON: total LAC energy greater than $8 \mathrm{GeV}$ and at least one central drift chamber track. Of the selected hadronic events, $99.9 \%$ satisfied the ENERGY trigger, of which $89 \%$ set the HADRON trigger, while only $34 \%$ satisfied the TRACK trigger. The total hadronic triggering efficiency has been estimated to be $95 \%$ [61]. In addition to the hadronic triggers, three other triggers were used in the run:

- BHABHA: at least $10 \mathrm{GeV}$ deposited in both north and south luminosity monitors;

- WAB (Wide-Angle Bhabha): total LAC energy greater than $30 \mathrm{GeV}$;

- RANDOM: fires continually at $0.05 \mathrm{~Hz}$.

The BHABHA and ENERGY triggers cause only the calorimetry system (LAC, warm-iron calorimeter, and luminosity monitor) to be read out, enabling a calorimetry-based analysis of all hadronic decays, while the remaining four triggers log the entire event.

\subsubsection{Event topologies}

Before discussing the details of $Z$ event selection, it is helpful to review the features of different types of $Z$ decays and their backgrounds.

\section{$\mathbf{Z}$ decays}

The $Z$ decay types of interest for the measurement of $A_{L R}$ are hadronic decays and $\tau^{+} \tau^{-}$events. Muon events deposit little energy in the LAC and are not included in the $A_{L R} Z$ sample, and Bhabha events are treated below as backgrounds.

Hadronic decays Hadronic decays of the $Z$ are distinguished by a large number (average $\sim 20$ ) of charged tracks and energy deposition of several tens of $\mathrm{GeV}$ in the detector. In addition, the energy of the event is well balanced about the interaction point. 
Tau decays Tau decays occur as single-track or multi-track events. Single-track events (one track for each $\tau$ ) in general deposit less energy than Bhabha events and can be unbalanced in energy. Multi-track events create a broader energy deposition pattern than Bhabha events, and, if tracking is used, are distinguishable by their greater multiplicity.

\section{Z backgrounds}

Several types of backgrounds are possible in SLC running. Some of these are due to real $e^{+} e^{-}$ interactions, while others are single-beam or cosmic-ray events. For negligibly rare processes, we estimate the size of the contamination below.

Wide-angle Bhabha events Wide-angle Bhabha events make up the largest source of background to the $1992 Z$ sample. Wide-angle Bhabha electrons typically deposit all of their energy in just one or two electromagnetic calorimeter towers. The events consist of two energy-balanced tracks each with the beam energy. Radiative Bhabhas, defined by $e^{+} e^{-} \rightarrow e^{+} e^{-} \gamma$, have a different topology and can be misidentified as $Z \mathrm{~s}$.

Two-photon events We define two-photon events as those mediated by two photons with $e^{+} e^{-} f \bar{f}$ in the final state. The $e^{+} e^{-}$in the final state generally travel unobserved down the beampipe, while the detected $f \bar{f}$ are characterized by low total energy deposition, balanced transverse momentum, and imbalanced longitudinal momentum. We use a two-photon Monte Carlo generator based on the equivalent-photon approximation in conjunction with the SLD detector simulation to find that at 95\% confidence level, an upper limit of three two-photon events are expected to exist in our sample. All three events are $e^{+} e^{-} \rightarrow e^{+} e^{-} e^{+} e^{-}$processes resembling wide-angle Bhabha events.

Two-gamma events With apologies for the weak notational distinction, we define two-gamma events as QED $t$-channel photon-photon final-state production. Using data from LEP to constrain deviations from QED in this process [62], we integrate the cross section over the LAC to find

$$
\sigma\left(e^{+} e^{-} \rightarrow \gamma \gamma\right)=61_{-9}^{+4} \mathrm{pb}
$$

approximately $0.2 \%$ of the total $Z$-peak cross section. The signature for the two-gamma process is two back-to-back $45 \mathrm{GeV}$ electromagnetic hits, just as for wide-angle Bhabhas. 
Photon-mediated and interference events At the $Z$ pole, the photon-mediated $s$-channel cross section is finite but dominated by the $Z$ resonance. In addition, $\gamma-Z$ interference has a small contribution off-pole. Including only hadronic and tau decays, the contribution of the $\gamma$ and $\gamma$ $Z$ events is $0.12 \%$ of the cross section at the $Z$ peak and $0.17 \%$ at the 1992 SLD run energy of $91.55 \mathrm{GeV}$. The latter fraction corresponds to 18 events in our sample, of which 5 are due to $\gamma-$ $Z$ interference. Our definition of $A_{L R}$ from Eq. 1.33 uses the cross section for $e^{+} e^{-} \rightarrow Z$; hence, we must treat the photon-mediated events as background in the $A_{L R}$ determination. The expected 5 interference events cannot strictly be considered background, as they have an intrinsic left-right asymmetry; due to the small number of events, however, the distinction is unimportant, and we include these events also as part of the background. For the determination of $\sin ^{2} \theta_{W}^{\text {eff }}$ from $A_{L R}$ using Eq. 1.35, the left-right asymmetry must include the photon-mediated and interference events, as the effects of these diagrams are included in the renormalization and definition of $\sin ^{2} \theta_{W}^{\text {eff }}$. The effect on $\sin ^{2} \theta_{W}^{\text {eff }}$ of including these events is a shift of $\delta \sin ^{2} \theta_{W}^{\text {eff }} \sim 2 \times 10^{-5}$, again entirely negligible.

SLC muons Off-energy particles in the tails of either beam can scrape the beampipe and produce high-energy muons, which then traverse the SLD longitudinally at large radius, parallel to the beamline. A single SLC muon depositing energy through ionization leaves $3-6 \mathrm{GeV}$ in the LAC. This small energy loss and the distinctive nonprojective tracks simplify recognition in the filter. A radiative muon interaction in the LAC, through bremsstrahlung, pair production, or photonuclear interactions, deposits $\sim 10 \mathrm{GeV}$ in 2-10 towers; thus, two such clusters can mimic a $Z$ decay.

Beam-gas and beam-wall interactions Beam-gas or beam-beampipe interactions occur when a particle from either beam strikes stray matter in the SLC vacuum or the beampipe itself. These events are exceedingly rare given the average beam current and high SLC vacuum. Beam-interaction events are distinguished by low total energy, large energy imbalance, and a displaced vertex in the $z$ direction, and hence are easy to identify. The simultaneous occurrence of one such event from each beam, however, could resemble a valid $Z$ decay. To study this possibility, we examine 35016 random- or luminosity-triggered events throughout the run, none of which is identified as a $Z$, and look for total deposited energy and tower clustering in the events. We find that an upper limit of $0.8 \pm 0.7$ events in the 1992 sample could result from independent simultaneous back-to-back beam-gas events, where the uncertainty is statistical. 
Cosmic rays Cosmic rays can deposit substantial energy in the LAC. The dominant cosmic-ray process leaving significant energy $(20 \mathrm{GeV})$ in the LAC is radiation by a muon of a hard photon. The total number of muons of energy $\geq 20 \mathrm{GeV}$ traversing the interaction point region during SLD triggering in the 1992 run is estimated to be 4800 . Of these, the total number radiating more than $20 \mathrm{GeV}$ in the $\mathrm{LAC}$ is expected to be $\leq 1.4$ during the run.

\subsubsection{Stage 1 filter}

In order to suppress random noise and nonprojective beam-related tracks, we exploit the LAC tower geometry described in Chapter 2 in our event selection. We define an energy $E_{T O T}$ which includes LAC electromagnetic towers only if both sections (EM1 and EM2) are hit, and includes LAC hadronic towers only if one of the four corresponding EM towers is hit. Such coincident hits are called "non-isolated" hits. We number each ring of LAC towers according to its polar angle, from $\Theta_{b i n}=0$ at $90^{\circ}$ in the center of the LAC barrel to $\Theta_{b i n}=48$ at $0^{\circ}$. The $e / \mu$ ratio of 0.69 , defined as the ratio of energy deposited per $\mathrm{cm}$ in the calorimeters by an electron to that of an equal-energy muon, is used to relate the LAC and WIC energies to the "muon" minimum-ionizing scale.

The energy-related quantities used in the selection cuts below include only towers above a threshold of $0,60,120$, and $120 \mathrm{ADC}$ counts for EM1, EM2, HAD1, and HAD2, respectively, where 1 ADC count in the EM (HAD) section corresponds to a 2.0 (5.4) $\mathrm{MeV}$ energy loss for a minimumionizing particle ("muon" scale). These thresholds are chosen to provide the best discriminating power against noise while preserving $Z$ event energy [63]. The outermost LAC rings at $\Theta_{b i n}=48$ are excluded from the energy sums due to high beam noise.

The stage 1 filter employs the following selection criteria:

1. $0<N_{L A C}<3000$, where $N_{L A C}$ is the number of LAC towers above readout threshold, set at 2, 3, 6, and $6 \mathrm{ADC}$ counts for EM1, EM2, HAD1, and HAD2. This requirement excludes high-noise background events caused by poor beam conditions. The tower readout threshold is an ENERGY trigger requirement.

2. $E_{L A C}>20 \mathrm{GeV}$, where $E_{L A C}$ is the sum of towers above threshold. This requirement selects $Z$ events over backgrounds, which typically have smaller energy deposition.

3. $E_{W I C}^{e n d}<12 \mathrm{GeV}$ and $E_{\text {ToT }}-E_{W I C}^{\text {end }}>9 \mathrm{GeV}$, where $E_{W I C}^{\text {end }}$ is the sum of the endcap WIC tower hits with less than $6000 \mathrm{ADC}$ counts $(12 \mathrm{GeV})$ and non-isolated hits. This requirement excludes the SLC muon events described above; these events deposit much of their energy in 
the WIC endcaps.

4. $E_{I M B}<0.9$, where $E_{I M B}$ is the energy imbalance in the LAC, defined as

$$
E_{I M B}=\frac{1}{E_{T O T}} \sqrt{\left(\sum E_{x}\right)^{2}+\left(\sum E_{y}\right)^{2}+\left(\sum E_{z}\right)^{2}}
$$

where $E_{x}, E_{y}$, and $E_{z}$ are the signed $x, y$, and $z$ components of $E_{T O T}$. This requirement also excludes SLC muons by identifying their large energy imbalance compared with that of $Z$ events.

5. $E_{I M B}+S P H E<1.0$, where $S P H E$ is the sphericity of the event, defined by

$$
S P H E \equiv \frac{3}{2} \frac{\sum_{i}\left|\vec{p}_{i} \times \hat{S}\right|^{2}}{\sum_{i}\left|\vec{p}_{i}\right|^{2}} ;
$$

the sum is over the individual track momenta $\vec{p}_{i}$ and the direction of the unit vector $\hat{S}$ is chosen to minimize SPHE. This criterion was developed for the 1991 engineering run to reject SLC muon events during severe beam backgrounds, and has a negligible effect on the 1992 data.

Figs. 4.1 and 4.2 show the progression of data reduction as the cuts are applied to four sample SLD runs.

These cuts were applied to the entire sample of triggered events in the 1992 data set. 13478 events passed this first-stage filter. Figs. 4.3 and 4.4 show the distributions of the events passing these cuts. In Fig. 4.4 we see a population of wide-angle Bhabha events at high $E_{T O T}$ and low $E_{I M B}$, while one-sided SLC backgrounds can be seen at low $E_{T O T}$ and high $E_{I M B}$.

\subsubsection{Stage 2 filter}

The second filter stage attempts to identify and remove wide-angle Bhabha events [64]. To accomplish this goal, we exploit the large localized electromagnetic energy deposition of Bhabha electrons. On average, a wide-angle Bhabha electron deposits $95 \%$ of its energy in only two towers in the LAC EM sections. We define the quantity $S_{4}$ as the sum of the two maximum-signal towers in each EM section with a $2 \mathrm{GeV}$ tower threshold. In addition, we define $\Theta_{\max }$ to be the $\Theta_{\text {bin }}$ of the tower with the maximum EM1 energy. Using the conversion $4.09 \mathrm{MeV} / \mathrm{ADC}$ count ("min i" $\times 2$ ), we define a wide-angle Bhabha as an event passing the following criteria:

1. $S_{4}>30$ for $\Theta_{\max } \leq 44$ 

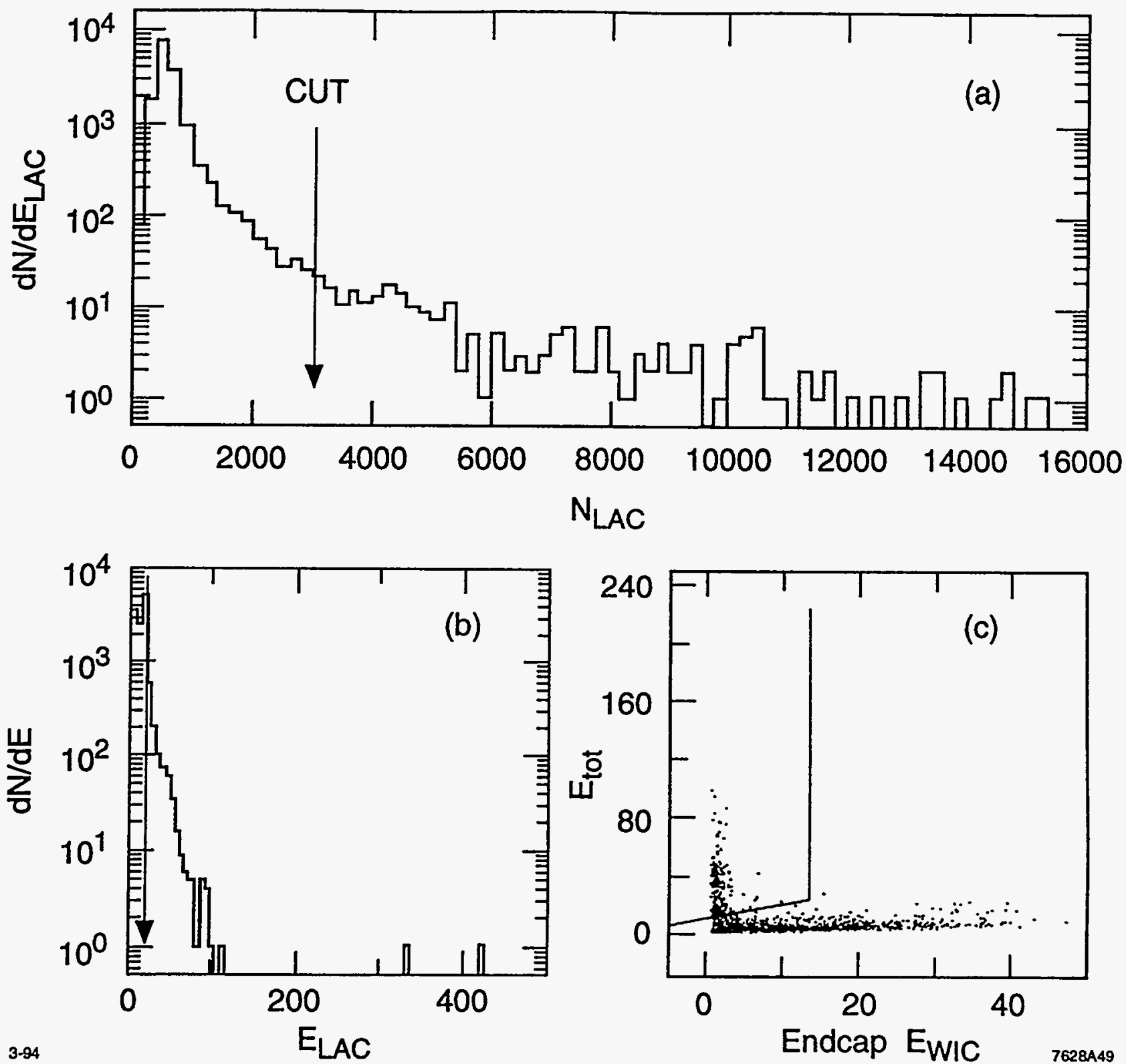

Figure 4.1: (a) Distribution of $N_{L A C}$ for all triggered events of four sample runs. (b) Distribution of $E_{L A C}$ for the runs. (c) $E_{T O T}$ vs. $E_{W I C}^{\text {end }}$ after cuts 1 and 2. 

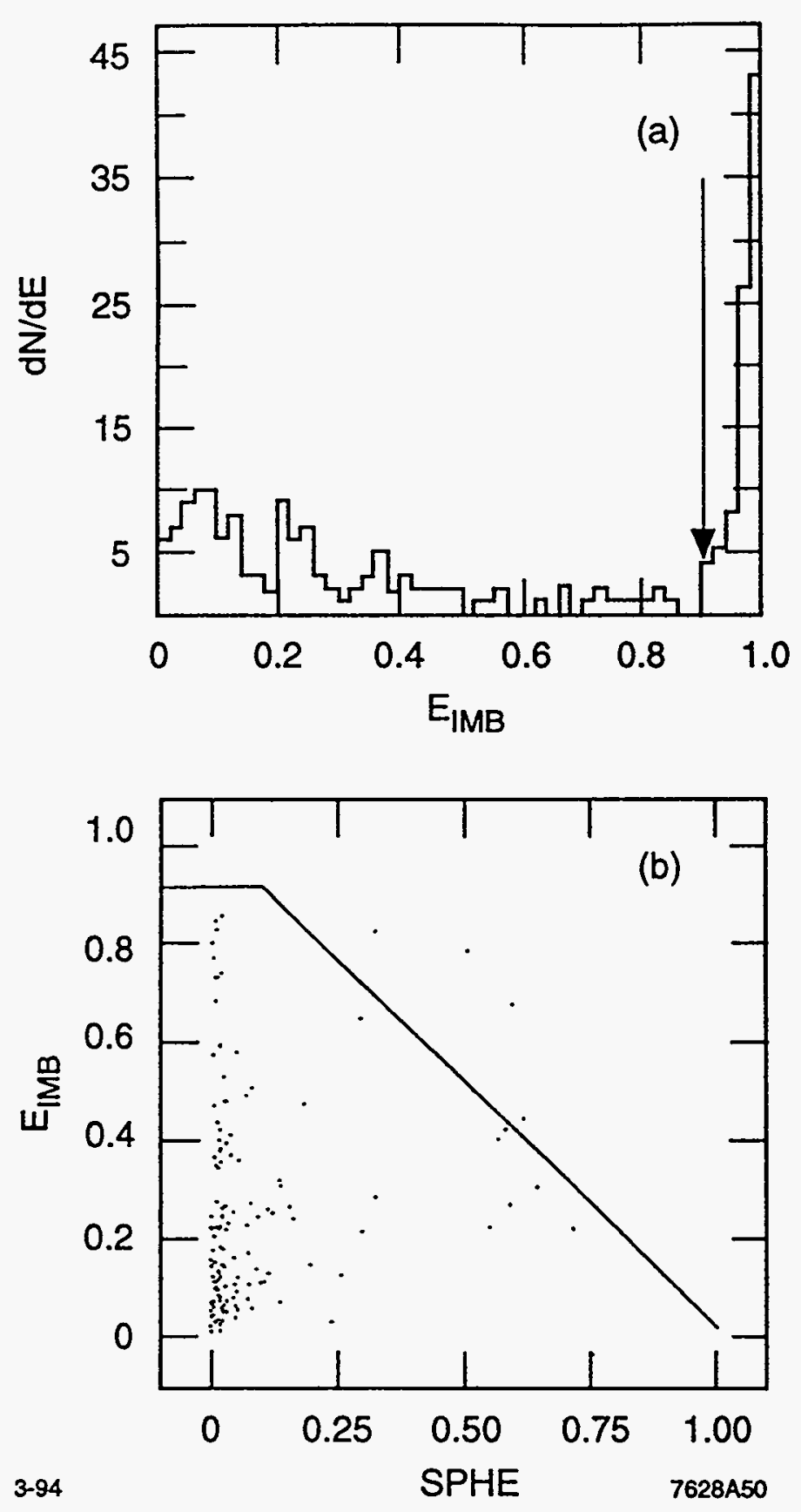

Figure 4.2: (a) Distribution of $E_{I M B}$ after cuts 1,2, and 3. (b) $E_{I M B}$ vs. $S P H E$ after cuts 1,2,3, and 4. 

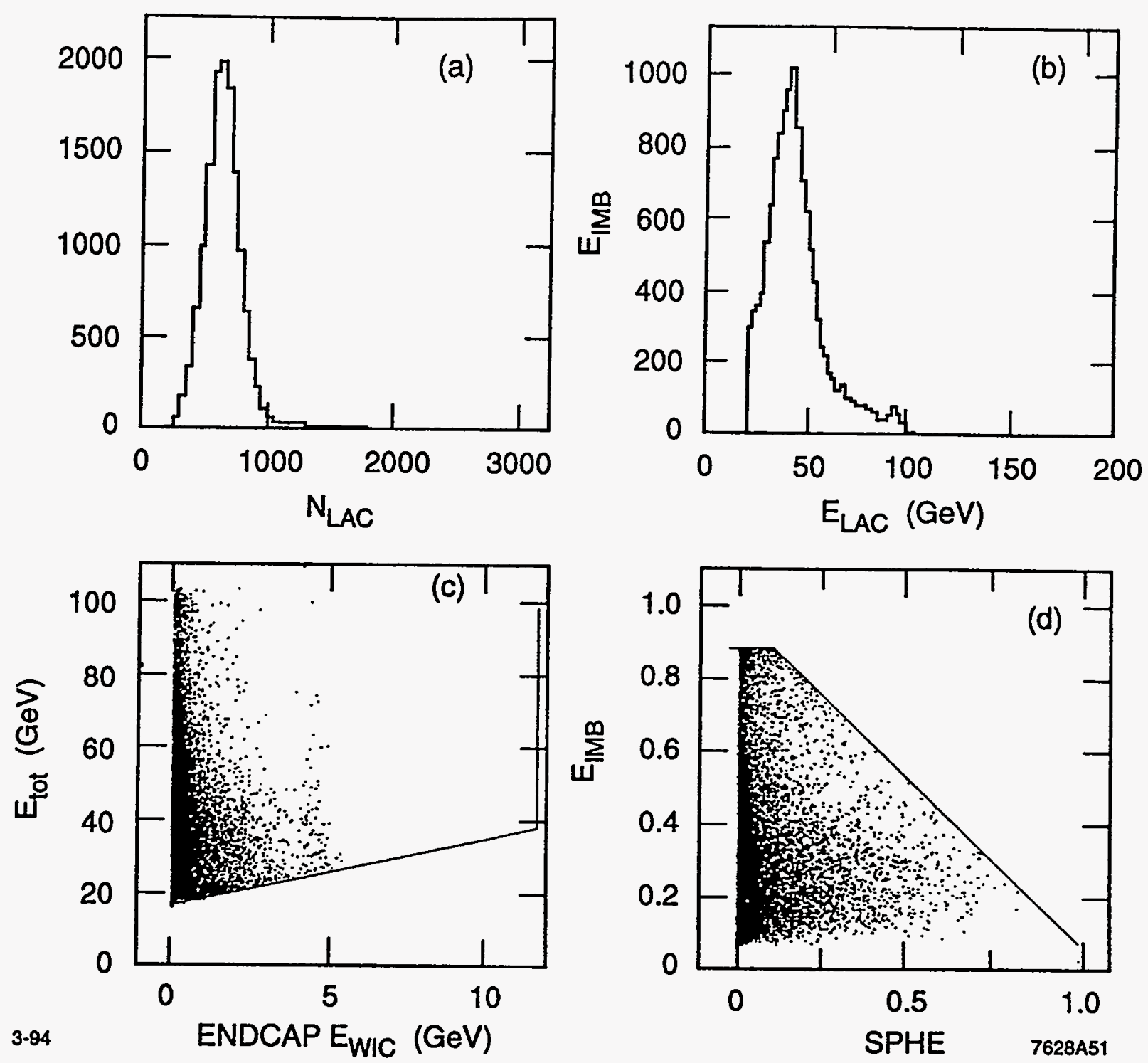

Figure 4.3: (a) Distribution of $N_{L A C}$ for events passing stage 1 filter. (b) Distribution of $E_{L A C}$ for these events. (c) $E_{T O T}$ vs. $E_{W I C}^{\text {end }}$ for the same events. (d) $E_{I M B}$ vs. SPHE for the events. 


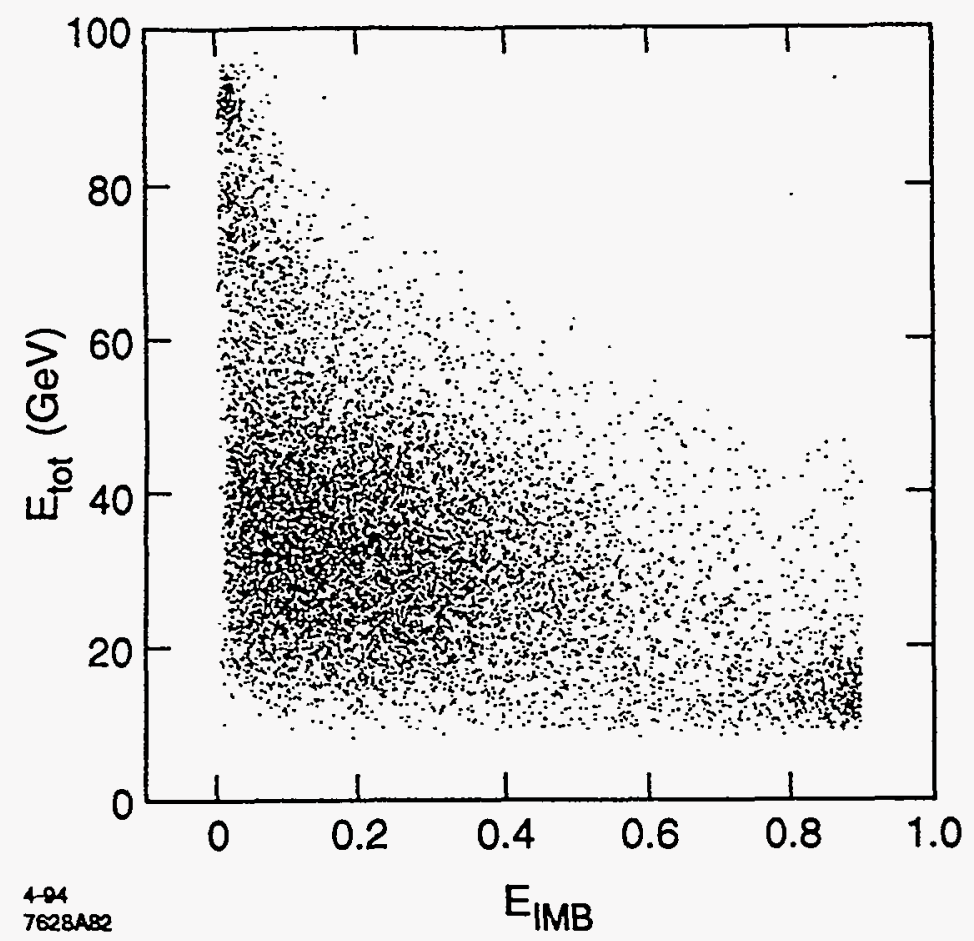

Figure 4.4: $E_{T O T}$ vs. $E_{I M B}$ for events passing stage 1 filter. Note wide-angle Bhabha-event population at upper left and beam-related backgrounds at lower right.

2. $S_{4}>12$ for $\theta_{\max }>44$.

Fig. 4.5 plots $S_{4}$ vs. $\Theta_{\max }$ for all events passing the stage 1 filter. The Bhabha energy degradation at small scattering angles seen the figure is likely due to increased preshowering in the endcap region, as well as increased energy leakage into the HAD section.

A total of 1865 events were identified by the stage 2 filter as wide-angle Bhabhas and were removed from the data sample. Fig. 4.6 shows the $E_{T O T}$ distribution of events passing the $\Theta_{\max } \leq 44$ cut. Barrel wide-angle Bhabhas populate a peak at $90 \mathrm{GeV}$, while endcap events create a lower-energy tail. Also shown is the distribution of events passing the $\Theta_{\max }>44$ cut, with the expected lower mean energy.

\subsubsection{Stage 3 filter}

The third-stage filter is intended to remove beam-related backgrounds, especially SLC-induced muons. Fig. 4.7 is the remainder of Fig. 4.4 after the second-stage filter has removed the wideangle Bhabhas at the top left. The cuts on $E_{T O T}$ and $E_{I M B}$ in the stage 3 filter are shown. We also define the quantities $M 1$ and $M 2$, the maximum tower energies in the EM layers for the forward 


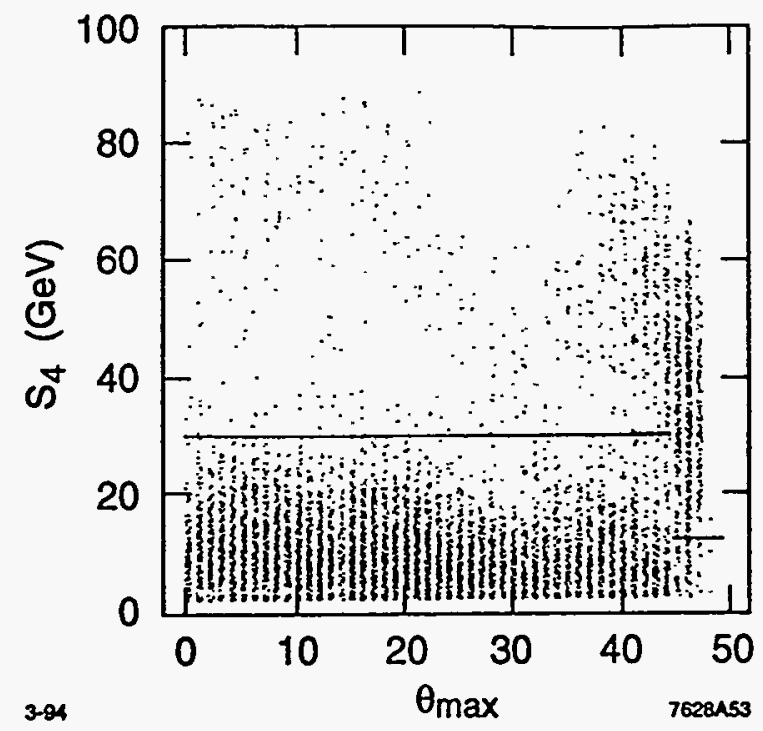

Figure 4.5: $S_{4}$ vs. $\Theta_{\max }$ for all events passing stage 1 filter. The cuts of filter stage 2 are shown.

and backward hemispheres, respectively. Fig. 4.8 shows $\operatorname{Min}(M 1, M 2)$ plotted vs. $E_{I M B}$ for the same event sample and also shows the cuts on $\operatorname{Min}(M 1, M 2)$ and $E_{I M B}$ in this stage. The criteria composing the third-stage filter are:

1. $14.5<E_{T O T}<101 \mathrm{GeV}$ (the $e / \mu$ factor is used to correct the "min i" scale), as beam backgrounds are lower in energy than $Z$ events;

2. $E_{I M B}<0.8$, as explained in Stage 1;

3. $\operatorname{Min}(M 1, M 2)>0.5 \mathrm{GeV}$, to ensure that both sides of the event have at least one energetic track, eliminating soft one-sided beam noise. We use here the conversion $4.09 \mathrm{MeV} / \mathrm{ADC}$ count ( $\min i " \times 2)$.

A total of 1176 events were identified as background in the third-stage filter and were rejected. The remaining 10437 events compose the final $Z$ data sample. Fig. 4.9 shows the distributions of $E_{T O T}, E_{I M B}$, and $M i n(M 1, M 2)$ for the event sample.

An alternative hadronic event selection procedure [65] finds 10002 hadronic events in the 1992 run. This selection makes use of calorimetry reconstruction to form LAC hit clusters. 340 of these events fail to pass the "official" filter. A hand scan of these events, using the same criteria as for the background estimation discussed below [60], indicates that 320 of them are good hadronic or tau events. Thus, the official selection procedure is $97 \%$ efficient for finding hadronic and tau events in the alternative sample. When the official filter is applied to the alternative event sample, 400 of the 

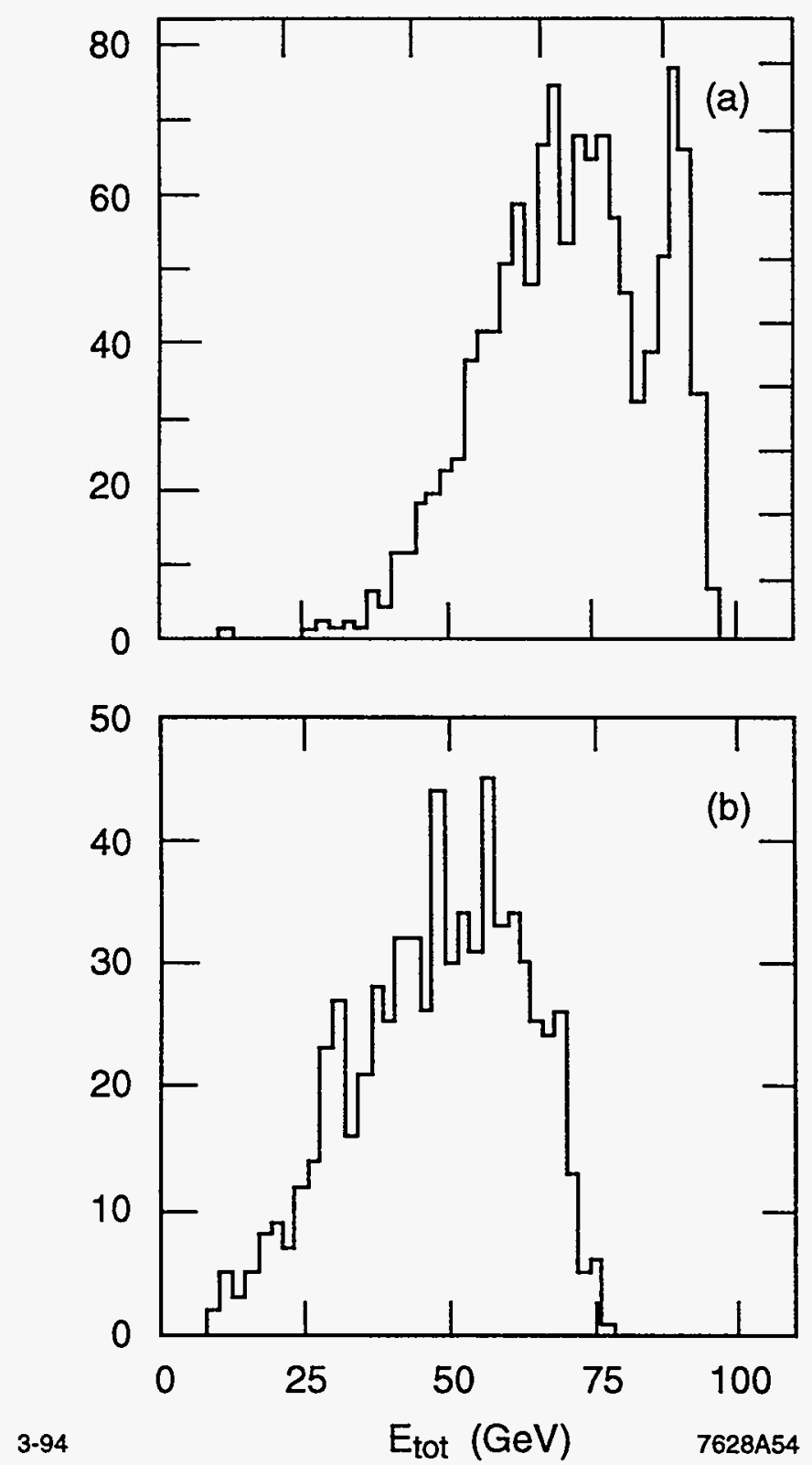

Figure 4.6: (a) Distribution of $E_{T O T}$ for events identified by second-stage filter as wide-angle Bhabhas in the region $\Theta_{\max } \leq 44$. (b) Distribution for those wide-angle Bhabhas with $\Theta_{\max }>44$. 


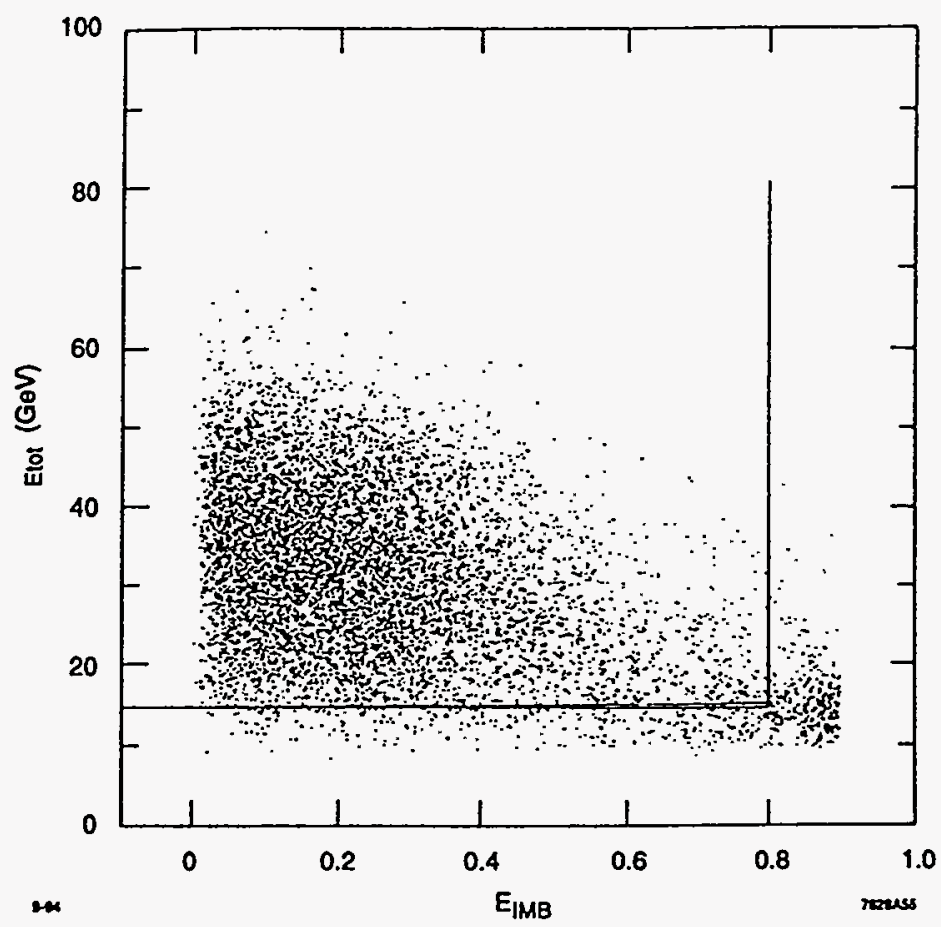

Figure 4.7: $E_{T O T}$ vs. $E_{I M B}$ for all events passing stage 2 filter. The cuts in stage 3 on $E_{T O T}$ and $E_{I M B}$ are shown.

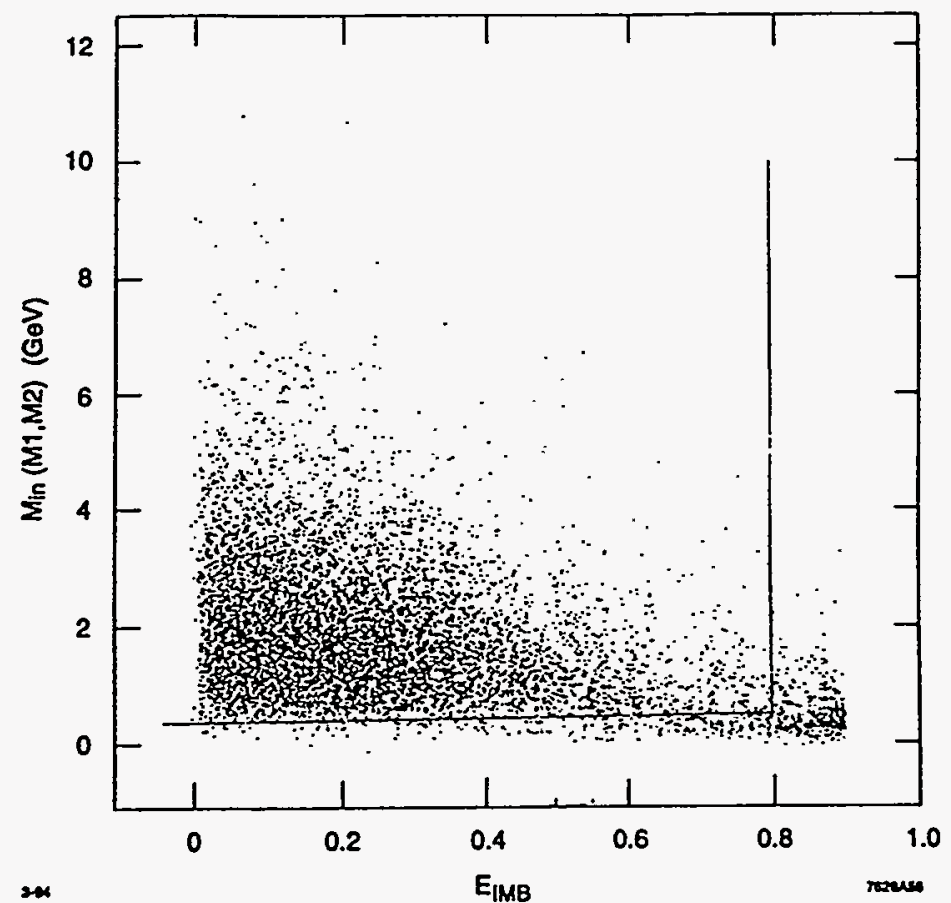

Figure 4.8: $\operatorname{Min}(M 1, M 2)$ vs. $E_{I M B}$ for the same sample. The stage 3 cuts on $\operatorname{Min}(M 1, M 2)$ and $E_{I M B}$ are shown. 

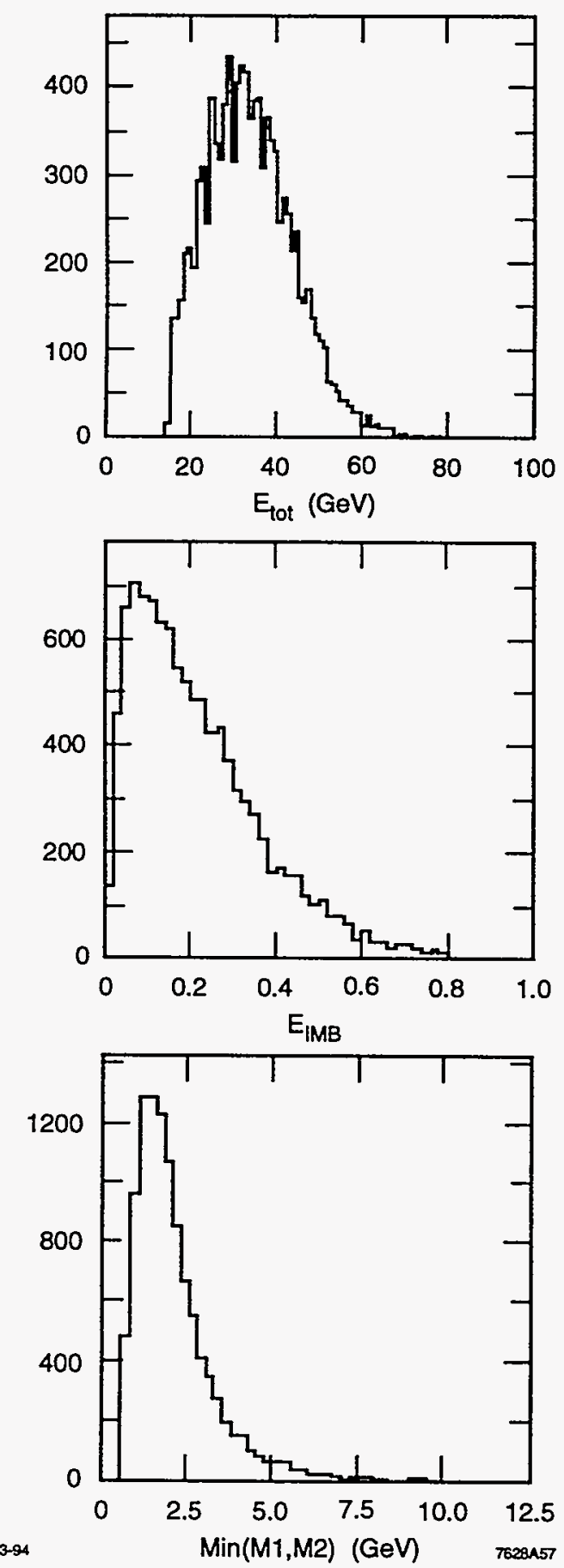

Figure 4.9: Top: Distribution of $E_{T O T}$ in the final event sample. Center: Distribution of $E_{I M B}$. Bottom: Distribution of $\operatorname{Min}(M 1, M 2)$. 
10437 events fail to pass. A hand scan estimates that 280 of these events are valid hadronic or tau events.

\subsubsection{Polarization-related cuts}

Having selected a sample of $Z$ events, we now make additional requirements on the understanding of the beam polarization at the time of each $Z$. The remaining cuts belong to two categories: dataquality cuts and polarization-time cuts. The data-quality criteria are:

1. Klystron Veto Module data is uncorrupted (i.e., transmitted data-integrity bit is good);

2. Independent offline data banks agree on beam-crossing identification number for each $Z$;

3. Source Pockels cell is within tolerance according to Klystron Veto Module information.

This set of cuts removes 120 events, due to KVM data-integrity failures, reducing the $Z$ sample to 10317 events. The polarization time cut ensures that the polarization associated with $Z \mathrm{~s}$ is representative of the true beam polarization at the time of creation of the $Z$. This criterion is:

1. Measurement of the beam polarization has been performed within one hour of each event.

This requirement removes 93 more $Z \mathrm{~s}$, generally due to operator error in failing to restart polarimeter running after an interruption.

\subsubsection{Measured asymmetry}

The final $A_{L R}$ data sample comprises 10224 events. Examination of the electron-bunch helicity for each $Z$ results in a total of 5226 events created with left-handed beam and 4998 events created with right-handed bearn. The understanding of the relationship between helicity at the source and that at the IP will be discussed in the final chapter. The result for the measured $Z$ asymmetry $A_{m}$ is then

$$
A_{m} \equiv \frac{N_{L}-N_{R}}{N_{L}+N_{R}}=(2.23 \pm 0.99) \times 10^{-2}
$$

where the uncertainty is statistical.

\subsection{Background estimation}

The selected $Z$ sample inevitably contains some non- $Z$ events. If the background has no significant asymmetry, the only effect of these events is a proportional dilution of $A_{L R}$; however, the size of 
the background must be accurately estimated to know the magnitude of the correction and error to apply to $A_{L R}$. We have discussed the possible sources of background earlier in the chapter; here we assess the size of the contamination. Details of this analysis are found in [60].

\subsubsection{Background estimation procedure and results}

In order to exploit the only advantage offered by poor luminosity, the decision was made to hand scan the entire $Z$ event sample. The hand scan was carried out by two physicists operating independently but using the same set of criteria for all possible types of signal and background events. The fraction of the sample considered to be background was separated into two groups: nonresonant $e^{+} e^{-}$events and beam and cosmic events. The results of the scan are shown in Table 4.1. The first two columns contain the total number of background events, separated by type, seen by each scanner $\left(N_{1}\right.$ and $\mathrm{N}_{2}$ ), while the third column is the number of these events identified by both scanners as background $\left(n_{12}\right) \cdot{ }^{1}$

\begin{tabular}{|c|c|c|c|}
\hline Background type & Scanner \#1 $\left(\boldsymbol{N}_{\mathbf{1}}\right)$ & Scanner \#2 $\left(\boldsymbol{N}_{\mathbf{2}}\right)$ & Overlap $\left(\boldsymbol{n}_{\mathbf{1 2}}\right)$ \\
\hline Nonresonant & 75 & 88 & 49 \\
\hline Beam-related & 71 & 72 & 64 \\
\hline All backgrounds & 146 & 160 & 115 \\
\hline
\end{tabular}

Table 4.1: Results of the two independent hand scans. The first two columns represent numbers of background events identified by each scanner, while the third column contains the intersection of the first two columns.

Using the results of the scan, we employ two different analysis methods to extract an estimate of the true background fraction. The first assumes that the scans are performed independently and that all backgrounds have the same probability to be detected. It is straightforward to show in this case that the number of background events $N$ is given by

$$
N=\frac{N_{1} N_{2}}{n_{12}}
$$

with $N_{1}, N_{2}$, and $n_{12}$ as defined earlier, and with statistical error $\delta N$ given by

$$
\delta N=N\left(\frac{1}{N_{1}}+\frac{1}{N_{2}}+\frac{1}{n_{12}}\right)^{\frac{1}{2}} .
$$

\footnotetext{
${ }^{1}$ The slight difference between the sum of the first two rows and the final row of the last column is due to two events that Scanner \#1 identified as beam-related and Scanner \#2 as nonresonant.
} 
The second method develops a likelihood function for the number of background events. The assumptions of this method are that the scans are not $100 \%$ efficient, implying that the systematic error in the background count is greater than the statistical error, and that the efficiency of both scanners to find a class of background events is constant for all types of background. We can then obtain the binomial probability for a scanner to find $N_{1}$ background events in $N$ total background events, the probability for the second scanner to find $n_{12}$ background events in these $N_{1}$ events, and the probability for the second scanner to find his remaining $N_{2}-n_{12}$ events. Combining these probabilities and solving the resulting likelihood function, we apply the data in Table 4.1 to obtain the graph in Fig. 4.10.

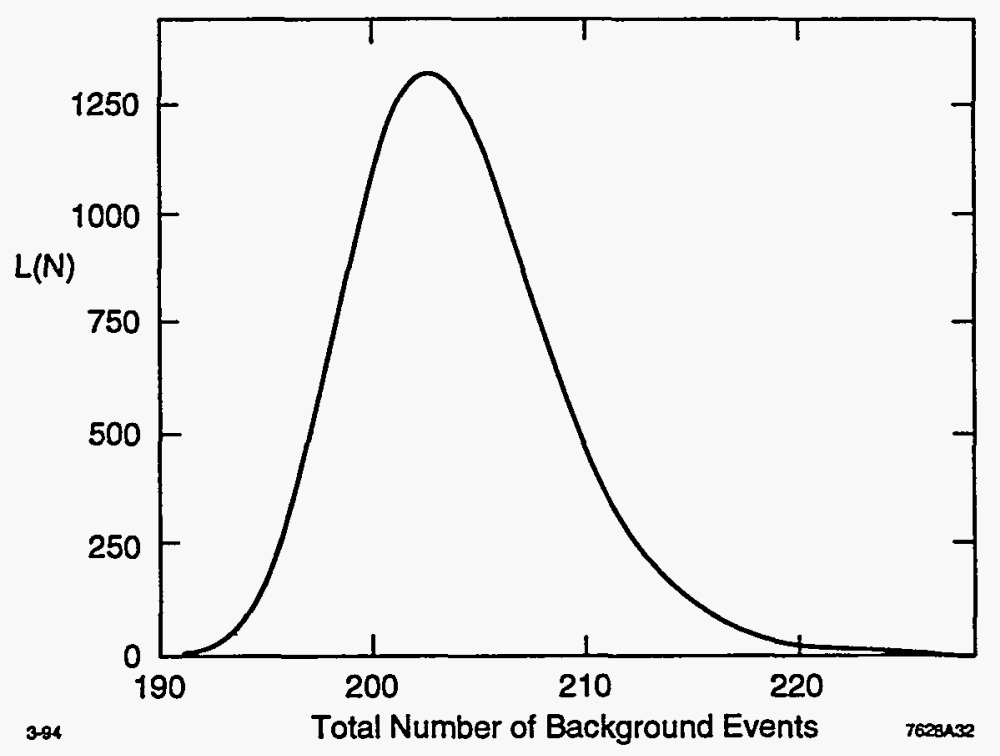

Figure 4.10: Likelihood function for number of background events. Vertical scale is arbitrary.

The results of the two background estimation techniques are listed in Table 4.2. The uncertainties in the maximum-likelihood method numbers are at $95 \%$ confidence level. We also include the estimates of the background contribution due to back-to-back beam-interaction events and to cosmic rays, as well as the expected component due to $\gamma$-mediated and $\gamma-Z$ interference events. The total background estimates from the two methods are in agreement, with the maximum-likelihood technique giving smaller errors. In assessing an error to the background estimate, we recall that both estimation methods rely on untested assumptions regarding scan probabilities and efficiencies for different background types. Wishing to err on the side of conservatism, we take as the uncertainty all background events found by only one scanner, or $N_{1}+N_{2}-2 n_{12}=76$ events. The total $Z$ sample 
background for the 1992 run is determined to be $223.2 \pm 76$ events, or $(2.1 \pm 0.7) \%$ of the sample. The systematic error in $A_{L R}$ resulting from this background will be discussed with the other uncertainties in the next chapter.

\begin{tabular}{|c|c|c|c|}
\hline Analysis technique & Background type & $\boldsymbol{N}$ & \% of sample \\
\hline \multirow{3}{*}{ Efficiency } & Nonresonant & $135 \pm 29$ & 1.3 \\
\cline { 2 - 4 } & Beam-related & $80 \pm 17$ & 0.8 \\
\cline { 2 - 4 } & All backgrounds & $203 \pm 30$ & 1.9 \\
\hline \multirow{2}{*}{ Maximum-likelihood } & Nonresonant & $133_{-12}^{+19}$ & 1.3 \\
\cline { 2 - 4 } & Beam-related & $80_{-0}^{+3}$ & 0.8 \\
\cline { 2 - 4 } & All backgrounds & $202_{-7}^{+12}$ & 1.9 \\
\hline \hline Double beam-gas estimate & Beam-related & $0.8 \pm 0.7$ & 0.008 \\
\hline Cosmic ray estimate & Cosmic & $<1.4$ & $<0.013$ \\
\hline$\gamma$ and $\gamma-Z$ & Nonresonant & 18 & 0.17 \\
\hline Total estimated background & & $223.2 \pm 76$ & $2.1 \pm 0.7$ \\
\hline
\end{tabular}

Table 4.2: Results of background estimation techniques and expected numbers of misidentified $Z \mathrm{~s}$.

The $Z$ sample selected with the alternative procedure of Reference [65] was subjected to the same scanning method and criteria. The background in the alternative sample is found to be $63 \pm 31$ events.

\subsubsection{Background asymmetry}

As a consistency check, we examine the events determined in the hand scan to be background for a statistically significant left-right asymmetry. It is conceivable, though improbable, that SLC backgrounds could differ measurably for left- and right-handed electron bunches. In addition, tau decays misidentified as backgrounds or wide-angle Bhabhas mediated by $Z$ bosons contribute to a background asymmetry.

Table 4.3 lists the observed background events by electron bunch helicity. Twelve of the 192 events had no associated polarization measurement within one hour of the event. The remaining 180 events have an asymmetry $A_{m}^{b}=0.078 \pm 0.074$, where the error is statistical. This large error in $A_{m}^{b}$ can be avoided by examining the background events obtained during random SLD triggers. This study reveals a negligible asymmetry in the background. For this reason, and because the above 
background asymmetry is consistent with zero, we do not correct the extracted value of $A_{L R}$, nor do we include this statistical error in our measurement.

\begin{tabular}{|c|c|c|c|c|}
\hline Background type & Total number & $N_{L}$ & $N_{R}$ & $A_{m}^{b}$ \\
\hline Nonresonant & 106 & 57 & 49 & $0.075 \pm 0.097$ \\
\hline Beam-related & 74 & 40 & 34 & $0.081 \pm 0.12$ \\
\hline All backgrounds & 180 & 97 & 83 & $0.078 \pm 0.074$ \\
\hline
\end{tabular}

Table 4.3: Left-right asymmetry in identified background events. Errors are statistical. 


\section{Chapter 5}

\section{Systematic Error Evaluation and}

\section{Results}

A number of systematic effects must be considered in calculating the value of $A_{L R}$ and its uncertainty. The dominant systematic error, in the measurement of the beam polarization, has been discussed in Chapter 3. In this chapter we evaluate the secondary systematic uncertainties in the measurement of $A_{L R}$, as well as several checks on the experimental procedure, including verification of the overall sign of the asymmetry. Finally, we present the results of the measurement and expectations for the future.

\subsection{Secondary Systematic Errors}

The expression 1.36 relating $A_{L R}$ to the measured asymmetry $A_{m}$ represents the "ideal" situation in the absence of $Z$-sample backgrounds and left-right asymmetries in the polarization, center-ofmass energy, detector acceptancexefficiency, and luminosity. All of these experimental imperfections skew the value of the measured asymmetry and require evaluation. The complete equation for $A_{L R}$ including these effects is given by

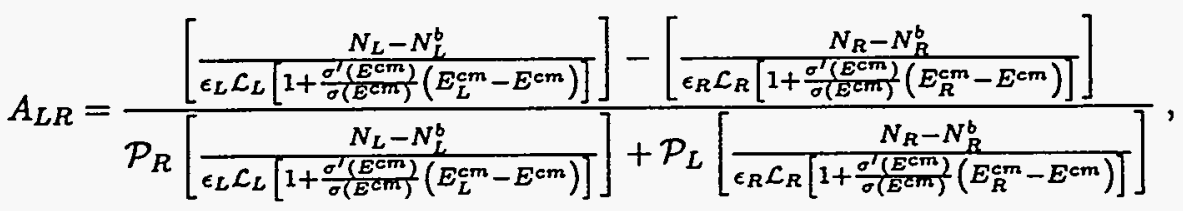


where $E^{\mathrm{cm}}$ is the mean center-of-mass energy averaged over left- and right-handed electron beams, $\sigma\left(E^{\mathrm{cm}}\right)$ is the total $Z$ cross section at energy $E^{\mathrm{cm}}, \sigma^{\prime}\left(E^{\mathrm{cm}}\right)$ is the energy derivative of the $Z$ cross section evaluated at $E^{c m}$, and $N_{L, R}, N_{L, R}^{b}, \epsilon_{L, R}, \mathcal{L}_{L, R}, E_{L, R}^{c m}$, and $\mathcal{P}_{L, R}$ are the total number of events, total background, detector acceptance-efficiency product, luminosity, mean center-of-mass energy, and luminosity-weighted mean polarization with left- and right-handed beam. Keeping terms only to first order in these small effects and rewriting in terms of left-right asymmetries, we find

$$
A_{L R}=\frac{1}{\mathcal{P}}\left[A_{m}+A_{m} f_{b}+A_{m}^{2} A_{\mathcal{P}}-E^{c m} \frac{\sigma^{\prime}\left(E^{c m}\right)}{\sigma\left(E^{c m}\right)} A_{E}-A_{\epsilon}-A_{\mathcal{L}}\right]
$$

where $f_{b}$ is the background fraction in the $Z$ event sample and $A_{\mathcal{P}}, A_{E}, A_{\epsilon}$, and $A_{\mathcal{L}}$ are the left-right asymmetries in the polarization, center-of-mass energy, detector acceptance-efficiency product, and luminosity.

\subsubsection{Background fraction}

The $Z$ sample background has already been discussed in Chapter 4, with the result $f_{b}=(2.1 \pm 0.7) \%$. The fractional correction to $A_{L R}$ due to the background is thus

$$
\left.\frac{\Delta A_{L R}}{A_{L R}}\right|_{f_{b}}=(2.1 \pm 0.7) \%
$$

\subsubsection{Polarization asymmetry}

The average beam polarization of a particular electron helicity can be analyzed by examining beam crossings with electron pulses of only that handedness; since these crossings are divided equally by photon helicity state, we can find the electron polarization by forming an asymmetry with the photon state. The run-averaged left- and right-handed polarizations found in this way are $\mathcal{P}_{L}=$ $0.2230 \pm 0.0002$ and $\mathcal{P}_{R}=0.2244 \pm 0.0002$, giving a polarization asymmetry of $A_{\mathcal{P}}=-2.95 \times 10^{-3}$. The fractional correction to $A_{L R}$ arising from this asymmetry is

$$
\left.\frac{\Delta A_{L R}}{A_{L R}}\right|_{A_{\mathcal{P}}}=-0.0066 \%
$$

with negligible uncertainty. 


\subsubsection{Energy asymmetry}

A large energy difference between left- and right-handed beams, combined with an off-peak average center-of-mass energy, can induce a cross section asymmetry between the two beam states due to the sharp falloff of the $Z$ resonance. The energy asymmetry in the 1992 run could not be measured directly with the energy spectrometers, as spectrometer data was not binned by beam helicity. Instead, in the expectation that the largest effect is due to beam loading in the linac, we examine the electron bunch-size asymmetry using information from the SLC current toroids. This asymmetry, averaged over the run, is found to be $(1.29 \pm 0.55) \times 10^{-4}$. With the conversion $400 \mathrm{MeV}$ lost in the linac per $10^{10}$ bunch electrons [66], we obtain an energy asymmetry of $(-7.1 \pm 2.5) \times 10^{-6}$. Using the run-averaged center-of-mass energy of $91.55 \mathrm{GeV}$ and evaluating the ratio $\sigma^{\prime}\left(E^{\mathrm{cm}}\right) / \sigma\left(E^{\mathrm{cm}}\right)$ at this energy, we find a fractional $A_{L R}$ correction of

$$
\left.\frac{\Delta A_{L R}}{A_{L R}}\right|_{A_{E}}=(0.076 \pm 0.027) \%
$$

\subsubsection{Acceptance-efficiency product asymmetry}

The SLD detector can introduce an artificial left-right asymmetry by preferentially accepting $Z$ s made with a particular beam handedness. The only conceivable source of such an effect is through a difference in the detector's response to fermions and antifermions. Because $Z s$ decay into back-to-back fermion-antifermion pairs, the fermionic angular distribution of, e.g., a "left-handed $Z$ " is the polar opposite of its antifermionic angular distribution. Since the fermionic and antifermionic angular distributions of a right-handed $Z$ are polar opposite to those of a left-handed $Z$, the detector will view the difference between the handednesses as a difference in local response to matter and antimatter. It can be shown that $A_{\epsilon}$ vanishes if either of the following conditions holds for the SLD detector:

1. Acceptancexefficiency for fermions must equal that for antifermions at every point on the detector, but may vary over the detector;

2. Acceptancexefficiency for fermions and antifermions must both be polar symmetric, averaging over azimuth, but may be different polar-symmetric functions.

These hypotheses have not been tested or accurately evaluated; however, the azimuthal symmetry of the SLD solenoidal field implies equal detection efficiencies for matter and antimatter independent of local acceptance nonuniformities. In addition, the calorimeter is highly uniform and polar-symmetric. For these reasons, the $A_{\epsilon}$ correction term is expected to be negligible. 


\subsubsection{Luminosity asymmetry}

A difference in SLC luminosity with left- and right-handed electron beams affects the measured value of $A_{m}$ in the same way as a detector acceptancexefficiency asymmetry. The luminosity asymmetry $A_{\mathcal{L}}$ is directly measured with small-angle Bhabhas, as these proceed dominantly through the $t$ channel and hence have no intrinsic asymmetry. The asymmetry is found in this way to be $A_{\mathcal{L}}=$ $(1.9 \pm 6.2) \times 10^{-3}$, with the large statistical uncertainty due to the low integrated luminosity in the 1992 run. We can avoid this statistical limitation by expressing $A_{\mathcal{L}}$ in terms of other beam observables that can be measured with higher precision. The luminosity of a round-beam collider such as the SLC is given by

$$
\mathcal{L}=\frac{N^{+} N^{-} f}{2 \pi\left(\sigma_{+}^{2}+\sigma_{-}^{2}\right)} e^{-\Delta^{2} / 2\left(\sigma_{+}^{2}+\sigma_{-}^{2}\right)},
$$

where $N^{ \pm}$are the $e^{ \pm}$bunch populations, $f$ is the collision frequency, $\sigma_{ \pm}$are the transverse spot sizes, and $\Delta$ is the $e^{+} e^{-}$targeting offset. Assuming that $N^{-}, \sigma_{-}$, and $\Delta$ are only weakly correlated, we can write

$$
A_{\mathcal{L}}=A_{N^{-}}+A_{\sigma_{-}}+A_{\Delta}
$$

where

$$
\begin{aligned}
A_{N^{-}} & \equiv \frac{N_{L}^{-}-N_{R}^{-}}{N_{L}^{-}+N_{R}^{-}} \\
A_{\sigma^{-}} & \equiv-\frac{\sigma_{-}^{L}-\sigma_{-}^{R}}{\sigma_{-}^{L}-\sigma_{-}^{R}} \\
A_{\Delta} & \equiv-\frac{1}{2} \frac{\bar{\Delta}\left(\Delta_{L}-\Delta_{R}\right)}{\sigma_{+}^{2}+\sigma_{-}^{2}}
\end{aligned}
$$

The bunch population asymmetry, given earlier, is found to be $A_{N^{-}}=(1.29 \pm 0.55) \times 10^{-4}$, while the offset asymmetry is determined from beam-beam deflection scans to be $A_{\Delta}=(0.2 \pm 1.0) \times 10^{-4}$, averaged over the run. The spot size asymmetry could not be measured directly and continually throughout the run, as the measurement is made with wire scans; thus, we again write the asymmetry in terms of other measured quantities. The beamstrahlung energy emitted per $e^{+} e^{-}$collision along the electron direction, for small $\Delta$, is given by

$$
U_{B S M}^{-}=\frac{4}{3 \sqrt{\pi}} \frac{N^{-} N^{+2} r_{e}^{3} \gamma^{2} m_{e}}{\sigma_{-}^{2} z^{+}} \ln \left[\frac{\left(1+\sigma_{-}^{2} / \sigma_{+}^{2}\right)^{2}}{1+2 \sigma_{-}^{2} / \sigma_{+}^{2}}\right],
$$


where $r_{e}$ is the classical electron radius, $\gamma$ is the electron Lorentz factor, $m_{e}$ is the electron mass, and $z^{+}$is the positron bunch length [67]. Then for $\sigma_{-}=\sigma_{+}$, we can write the left-right beamstrahlung asymmetry as

$$
A_{B S M}=A_{N^{-}}-0.3 A_{\sigma_{-}}
$$

This run-averaged asymmetry has been measured with the south beamstrahlung monitor to be $A_{B S M}=(1.2 \pm 1.0) \times 10^{-4}$. We can now evaluate $A_{\mathcal{L}}$, obtaining

$$
A_{\mathcal{L}}=(1.8 \pm 4.2) \times 10^{-4}
$$

and thus we find for the fractional $A_{L R}$ correction

$$
\left.\frac{\Delta A_{L R}}{A_{L R}}\right|_{A_{L}}=(0.81 \pm 1.88) \%
$$

\subsection{Further Systematic Error Checks}

In addition to the evaluation of measurable systematic uncertainties, we must verify that several potential effects remain negligible. We also find the absolute sign of the asymmetry using several different techniques.

\subsubsection{SLD-Compton spin precession}

The measurement of $A_{L R}$ requires precise understanding of the beam polarization at the beam interaction point. Use of the Compton polarimeter to measure $\mathcal{P}_{z}^{e}$ is valid only if the longitudinal component of the beam polarization remains unchanged through the final focus region. Beam-beam interaction depolarization is expected to be small [33]; however, spin precession must be investigated. This test is performed by measuring the effect of quadrupole magnets and correctors on electron beam trajectories through the final focus, and converting the net bend angle into spin precession using Eq. 2.2. Comparison of beam deflection under nominal running conditions to that with the quadrupoles and correctors turned off reveals a net steering angle of $<100 \mu \mathrm{r}$, yielding a net precession angle of $<10 \mathrm{mr}$. The effect on longitudinal polarization is a change of $\delta \mathcal{P} / \mathcal{P}<5.4 \times 10^{-5}$. 


\subsubsection{Helicity bit transmission integrity}

Crucial to the measurement of $A_{L R}$ is the transmission of the electron helicity information from the source to the SLD. As the helicity is chosen pseudo-randomly, incorrect or off-cycle information will dilute or erase the measured asymmetry. As mentioned earlier, this helicity information travels from the PLS to the SLD data acquisition system along two independent paths: the Klystron Veto Module line and a dedicated cable. Comparison of the bits from the two lines indicates that disagreement is negligibly rare $(<0.01 \%$ of tested pulses $)$.

\subsubsection{Data acquisition synchronization}

A subtler issue is the synchronization of helicity information with $Z$ events in the SLD data acquisition. Again, off-cycle association of the helicity data with SLD triggering will result in zero measured asymmetry, due to the random electron helicity. Synchronization tests fall into two categories: beam-dumper/extinction tests and polarization calculation tests.

\section{Beam-dumper/extinction tests}

In this type of test, electrons of only one helicity are transported to the SLD and the triggered data is examined for wrong-helicity events. These tests were performed several times under different conditions [68].

In the beam-dumper tests, the electron beam was produced alone at $10 \mathrm{~Hz}$ with the fixed $12-$ pulse pattern of one right-handed pulse followed by 11 left-handed pulses. The left-handed pulses were prevented by a beam dumper from reaching the SLD, and a $4 \mathrm{GeV}$-threshold SLD trigger was used to detect beam noise in the transmitted bunches. The test was performed on three separate occasions. The left-right trigger results for the three runs are

Run \#1: 4584 R $64 \mathrm{~L}$

Run \#2 : 2014 R $25 \mathrm{~L}$

Run \#3: $1123 \mathrm{R} 10 \mathrm{~L}$

The left-handed pulses are due to LAC noise and cosmic rays; imposing an $8 \mathrm{GeV}$ minimum-energy 
cut yields

$$
\begin{array}{lcc}
\text { Run \#1: } & 4584 \mathrm{R} & 0 \mathrm{~L} \\
\text { Run \#2: } & 1008 \mathrm{R} & 0 \mathrm{~L} \\
\text { Run \#3: } & 554 \mathrm{R} & 0 \mathrm{~L}
\end{array}
$$

Therefore, the beam-dumper tests indicate that the arrival of the helicity bits at the SLD is synchronized with the beam correctly. However, since almost all triggers were with right-handed beam, the possibility remains that the SLD data acquisition is mixing LAC and helicity data from different events. The beam-extinction test was devised to address this concern.

In the beam-extinction tests, the electrons are again produced alone, but at $120 \mathrm{~Hz}$ and using the pseudo-random helicity generator. In the first such test, bunches of a particular helicity were preferentially extinguished by a factor of $\sim 500$ by producing linear light with the source Pockels cell for that handedness and passing the light through a crossed linear polarizer. In this way, either helicity could be produced separately. The same trigger was used as for the dumper tests. The raw results from two runs with, respectively, left and right beam extinguished are

$$
\begin{array}{lcc}
\text { Run \#1 : } & 2484 \mathrm{R} & 14 \mathrm{~L} \\
\text { Run \#2: } & 8 \mathrm{R} & 2209 \mathrm{~L}
\end{array}
$$

Using the $8 \mathrm{GeV}$ cut from the dumper tests gives the result

$$
\begin{array}{lcl}
\text { Run \#1 : } & 1880 \mathrm{R} & 0 \mathrm{~L} \\
\text { Run \#2 : } & 2 \mathrm{R} & 1479 \mathrm{~L}
\end{array}
$$

To answer the question of event mixing, the test was repeated with changes to the triggering and electron production. The SLD trigger was set to fire as fast as possible in synch with the SLC $120 \mathrm{~Hz}$ beam crossing rate, in practice attaining $\sim 10 \mathrm{~Hz}$ due to the $100 \mathrm{~ms}$ dead time of the detector, with no LAC energy requirement. In addition, the Pockels cell was allowed to operate normally, but a 
quarter-wave plate and linear polarizer were installed after the Pockels cell to extinguish all lefthanded pulses. In this way, both left- and right-handed triggers would be accepted equally, and the triggered events could be examined to ensure that no beam-induced LAC energy was present for lefthanded pulses and hence that LAC and helicity information was not being mixed for different events. Low beam current during the test produced little beam noise, and hence blurred the distinction between beam-on and extinguished-beam triggers. The raw results of the run were

\section{Run \#1: $2544 \mathrm{R} \quad 2467 \mathrm{~L}$}

The innermost ring of EM towers in the north endcap LAC was summed to provide detection of beam noise. A cut of $>100 \mathrm{ADC}$ counts on this quantity yields

\section{Run \#1: $733 \mathrm{R} 9 \mathrm{~L}$}

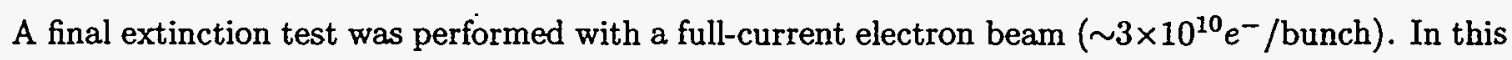
test, three overlayed random triggers were used to simulate nominal data-acquisition conditions. The usual $0.05 \mathrm{~Hz}$ random trigger was supplemented by a $0.1 \mathrm{~Hz}$ trigger reading the entire detector and a $0.5 \mathrm{~Hz}$ trigger reading the LAC only. The raw results from this test were

\section{Run \#1 : $1136 \mathrm{R} \quad 1188 \mathrm{~L}$}

Using a cut of 50 counts on the same LAC EM ring energy as above yields

Run \#1: $987 \mathrm{R} \quad 0 \mathrm{~L}$

These tests indicate that the expected failure rate of the data synchronization is less than $0.5 \%$ in all tests, and any measurable deviation from zero is almost certainly due to experimental shortcomings of the tests rather than true synchronization failure.

In addition to the dumper and extinction tests, a check was made of synchronization within the 
data acquisition by processing the direct-line helicity bits through a FASTBUS ADC in addition to the CAMAC path normally used. For all 2293 triggers written to tape in this mode, the klystron veto module bits, direct-line bits, and FASTBUS ADC bits were in $100 \%$ agreement.

\section{Polarization calculation test}

As a final check on SLD helicity-bit synchronization, the online beam polarization calculation was made using SLD helicity information along with Compton data [69]. The signal from channel 13 of the PTD detector was split and sent to an SLD CAMAC crate along with the Compton laser helicity signal. This data was then written to tape as part of the SLD data stream during triggered SLD events, which occurred at $\sim 0.5 \mathrm{~Hz}$, and was used to calculate the beam polarization for comparison with the standard Compton calculation. Due to the slow SLD trigger rate, the time required for a polarization measurement of $1 \%$ relative statistical precision was $\sim 11$ hours, over which period the true beam polarization typically varied by $2-3 \%$. The polarizations as calculated by the two methods agree within the $\delta \mathcal{P} \sim 1-2 \%$ statistical errors for all but two runs during the test. Examination of the PTD channel 13 signal for these runs reveals that the signal fluctuated and dropped sharply at these times due to beam and polarimeter conditions, and the decreased signal may have been further degraded in traversing the long cable to the SLD. Therefore, we believe that this check agrees with the previous tests in confirming correct SLD helicity-bit synchronization.

\subsubsection{Random number generator bias}

The pseudo-random number generator used to determine the helicity of the polarized electron bunches has been studied in detail [23] to evaluate its left-right bias. The generator is a 33-bit feedback shift register, producing a sequence of length $2^{33}-1$ before repeating, corresponding to 2.3 years of running at $120 \mathrm{~Hz}$. In practice, the sequence is reseeded approximately weekly. The sequence includes all possible 33-bit subsequences interleaved within it, each appearing once, with the exception of 33 consecutive zeroes. As the entire sequence can be calculated, the output can be predicted for all time beyond any known 33-bit subsequence. In addition, the generator is unbiased, producing equal numbers of ones and zeroes over the entire sequence. Shorter subsequences corresponding to those occurring at SLC have been investigated and no detectable bias has been found. Also, the sequence is polarized, so that a reversal of zero and one bits creates a different sequence that is detectable after 34 beam crossings. Such a reversal could result, for instance, from a cable swap at the source or at SLD. 
These properties allow a check of the integrity of the random number generator by determining the sequence phase during running and requiring agreement of the following bits with the predicted behavior. Since the Compton polarimeter ring buffer stores polarization data from 100 consecutive beam crossings at the end of each run, examination of the buffer reveals the sequence phase at that time. SLD triggers during each polarimeter run can then be checked for agreement of their helicity bits with the predicted values. As previously mentioned, the generator is sometimes reseeded, due to polarized source work. In addition, the SLD beam crossing number counter, used in this study to associate triggered events with the random sequence, was reset often. A set of long SLD runs was investigated to determine the frequency of unpredicted bit transmission. Wherever such a discrepancy occurs, the sequence was resynchronized to match the following data. Disregarding the SLD counter resets, which are independently observable, a total of three triggers of the $\sim 10^{5}$ in the set correspond to resynchronizations of the sequence. This frequency corresponds to a failure rate of $<0.003 \%$ at $95 \%$ confidence level and is consistent with expectations of intentional resets at the PLS. In addition, no bit reversals indicative of cable swaps are observed.

\subsubsection{Sign of asymmetry}

The Standard Model predicts that the cross section for $e^{+} e^{-} \rightarrow Z$ is greater for left-handed than for right-handed electrons, and hence that $A_{L R}$ is positive. We wish to find the sign of our measured asymmetry independently to confirm agreement with the prediction and ensure that we are not inadvertently missing a Nobel prize. The objective of this study is to determine the true helicity of the electron bunches that created the 5226 "left" $Z$ s and the 4998 "right" $Z$ s. This understanding is equivalent to knowledge of the sign of the theoretical Compton asymmetry and the relationship of the true laser light helicity and measured Compton asymmetry sign to the left-right beam labeling [57].

\section{Theoretical sign of Compton scattering}

While the cross section for polarized Compton scattering is well known and easily found in the literature, the absolute sign of the asymmetry is generally avoided and occasionally incorrect. A complete, simple argument has been provided us [70] for the purpose of checking the $A_{L R}$ measurement.

As we are concerned only with the sign of the asymmetry, we need consider only the completely backscattered case. In this case, the $t$-channel diagram of Fig. 3.1 dominates the $s$-channel diagram by a factor of $\sim 7$, due to the reduced energy in the Feynman denominator. Since the spin- $\frac{1}{2}$ electron enters and exits the interaction with $j_{z}= \pm \frac{1}{2}$ and the spin- 1 photon with $j_{z}= \pm 1$, and the total angular 
momentum of the system must remain constant, the only consistent possibility is that $j_{z}$ for both remain unchanged. Therefore, in the center-of-mass frame, where each particle reverses direction after the interaction, the electron helicity must flip in the process. The intermediate state of the $t$-channel process in Fig. 3.1 contains both photons as well as the intermediate (virtual) electron; thus, the total angular momentum in the intermediate state must equal $\pm 2 \pm \frac{1}{2}$. The initial and final total angular momentum, however, must equal $\pm 1 \pm \frac{1}{2}$, due to the real Compton photon and electron. Since spin must be conserved at each vertex, the initial and final angular momenta must equal the intermediate momentum, allowing only the $j_{z}= \pm \frac{3}{2}$ case in the process. Thus, the dominant helicity combination in Compton scattering is the parallel-spin $j_{z}= \pm \frac{3}{2}$ case.

\section{Photon helicity determination}

We must now determine the relationship between the true laser light helicity at the Compton IP and the helicity label in the data acquisition. Throughout this discussion, the phrases "right-handed" circularly polarized light and "positive-helicity" light refer to the photon state with spin along the particle direction; "left-handed" and "negative-helicity" light refer to the case with spin opposite the momentum. ${ }^{1}$ The laser pulses are assigned a bit pattern according to the state of the Compton Pockels cell. This relationship can be expressed in terms of a "Pockels cell sign" $S_{P C}$ as in Table 5.1. The helicity bit pattern 01 corresponds to positive high voltage on the Pockels cell, while the pattern

\begin{tabular}{|c|c|}
\hline Compton Laser Bit Pattern & True Helicity at Compton IP \\
\hline 00 & Unpolarized or no data \\
\hline 01 & $(+1) S_{P C}$ \\
\hline 10 & $(-1) S_{P C}$ \\
\hline 11 & Laser off \\
\hline
\end{tabular}

Table 5.1: Relationship between Compton laser bit pattern in data acquisition system and photon helicity at Compton IP.

10 denotes negative high voltage. Thus, the Pockels cell sign indicates the light helicity produced at the Compton IP using positive Pockels cell high voltage. Determination of $S_{P C}$ can be made in a number of ways; the chosen approach uses a quarter-wave plate in the arrangement shown in Figs. 5.1 and 5.2 [57]. With no phase shift in the Pockels cell (high voltage off), we orient the quarter-wave plate as in Fig. 5.2, so that the horizontal linear light produced by the initial linear polarizer emerges left-handed from the quarter-wave plate. We then apply positive quarter-wave

\footnotetext{
'This handedness convention is opposite to that in optics, where "right circularly polarized" light has negative helicity, i.e., has a clockwise-rotating electric field vector as viewed looking into the oncoming ray.
} 
voltage to the Pockels cell, creating circular light of positive or negative helicity incident on the quarter-wave plate. Left-handed incident light, corresponding to $S_{P C}=-1$, will exit the quarterwave plate linearly polarized vertically and will be extinguished by the final horizontal polarizer. Right-handed incident light, corresponding to $S_{P C}=+1$, will revert to horizontal linear light in the quarter-wave plate and will be passed to the screen by the final polarizer. Therefore, once the slow axis of the quarter-wave plate has been determined, the Pockels cell sign can be found.

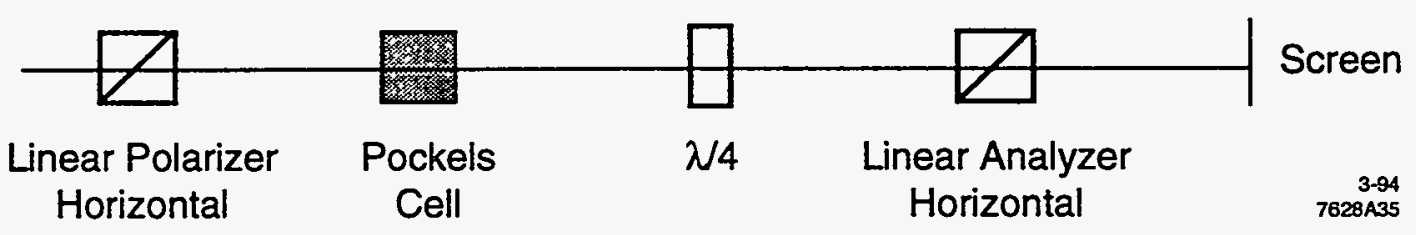

Figure 5.1: Experimental setup for test of Pockels cell sign. Laser light enters from the left.

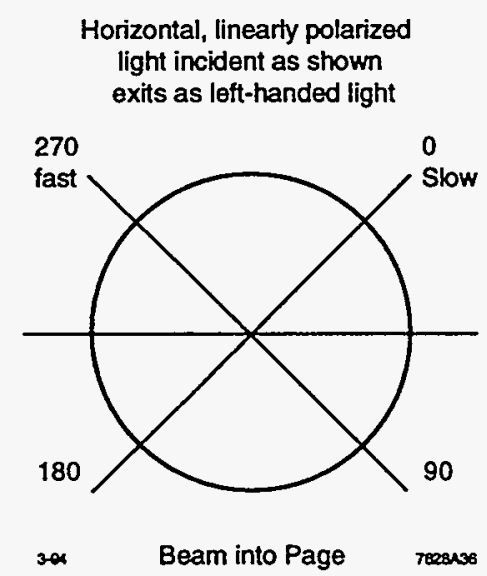

Figure 5.2: Orientation required of quarter-wave plate to convert horizontal linear light (into page) to left-handed light.

Determination of the quarter-wave-plate slow axis was made using several independent techniques [37]. The quarter-wave plates used at the Compton are marked by the manufacturer along the slow axis; it was decided to consider these markings as verification of our findings, if they agreed, rather than to accept their validity uncritically.

Fresnel technique The primary method of slow-axis determination exploits the possibility of generation of light of a particular known helicity, using a technique developed by Fresnel. Upon total 
internal reflection of polarized light, the component of linear polarization parallel to the plane of reflection undergoes a greater phase shift than the component perpendicular to the reflection plane [71]. Thus, the reflected light will become elliptically polarized with a known helicity. The relative phase shift $\delta$, where

$$
\delta \equiv \delta_{\perp}-\delta_{\|}
$$

is given by

$$
\tan \left(\frac{\delta}{2}\right)=-\frac{\cos \theta}{\sin ^{2} \theta} \sqrt{\sin ^{2} \theta-\frac{1}{n^{2}}}
$$

where $n$ is the index of refraction of the reflecting material and $\theta$ is the angle of incidence. For glass of $n=1.5$ and an incident angle of $45^{\circ}, \delta=37^{\circ}$; two successive reflections yield $\delta=74^{\circ}$, which approximates the $90^{\circ}$ phase shift of a quarter-wave plate. The experimental setup is shown in Fig. 5.3. The Compton laser shines through a linear polarizer, producing horizontal linear light, which then traverses a half-wave plate oriented to rotate the polarization plane by $45^{\circ}$ as shown in Fig. 5.4. Two successive total internal reflections produce nearly circular left-handed light. The quarter-wave plate is then rotated to produce maximal extinction of the beam. The slow-axis orientation producing extinction of left-handed light is given in Fig. 5.4. In this manner, the manufacturer's mark was confirmed on the slow axis of both Compton quarter-wave plates, and the slow axis was found on the tested PLS quarter-wave plate.

Liquid crystal polarizer technique Certain liquid crystals convert linearly polarized light into circular light of a particular helicity [72]. Commercially available optical elements employing this phenomenon exist; we used two such liquid crystal polarizers (LCPs), one frequency-optimized for the Compton laser and the other for the source laser, as a check on the Fresnel method described above. The LCPs used transmit right-handed light preferentially to left-handed light, reflecting the latter, with a measured extinction ratio of several hundred. One can simply check the slow-axis positions of the quarter-wave plates by making left- and right-handed circularly polarized light with the quarter-wave plates and observing reflection and transmission, respectively, with the LCP. These tests yield agreement with the Fresnel-method determination of the slow axis of all Compton and PLS quarter-wave plates.

Circular dichroism technique Some transparent materials preferentially transmit circularly polarized light of a particular helicity. This property, known as circular dichroism, is well known and exploited extensively by chemists. The circular dichroism (CD) of a material is parametrized by the 


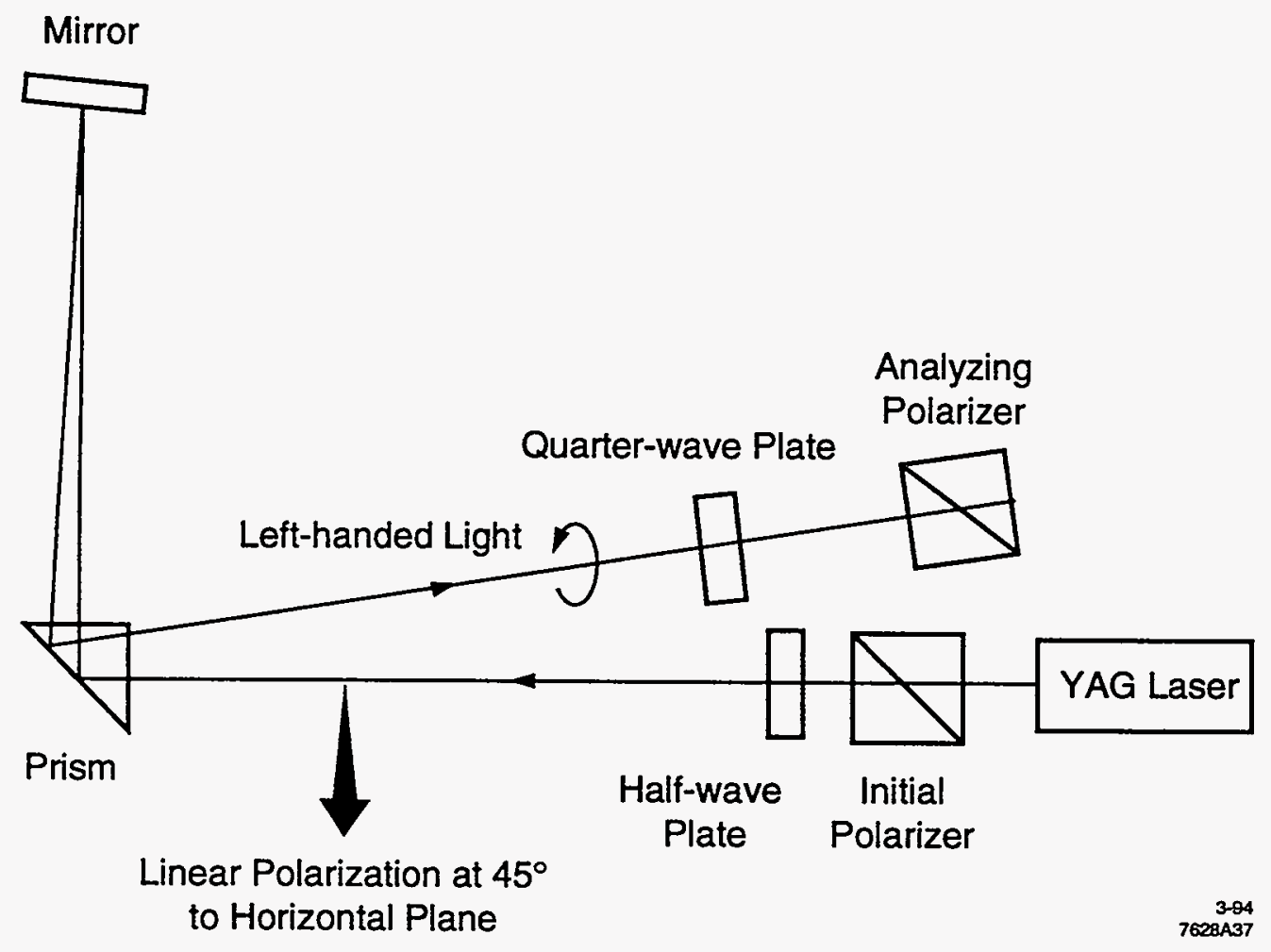

Figure 5.3: Experimental setup for Fresnel-method slow-axis determination.
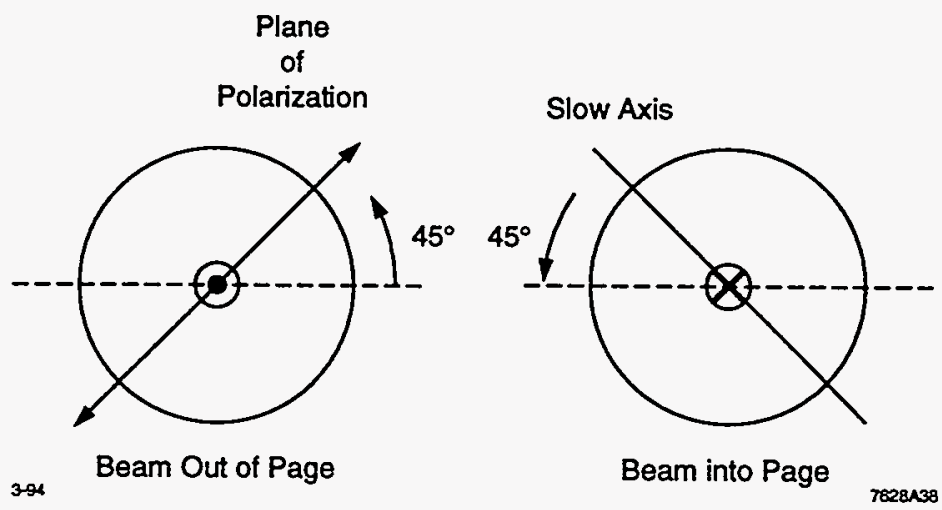

Figure 5.4: Left: Linear polarization orientation before total internal reflection. Right: Quarterwave-plate orientation for extinction. 
difference in absorption coefficients for the two helicities:

$$
\Delta A=A_{R}-A_{L}
$$

This definition gives $\Delta A>0$ for preferential attenuation of right-handed light. Access to a commercial CD spectropolarimeter was obtained to provide independent confirmation of the Fresnel method. The spectropolarimeter compares transmission through an inserted sample for left- and right-handed light for a range of wavelengths. The device was first calibrated by measuring the CD spectrum for aqueous cyano-cobalamin (vitamin B-12) and confirming agreement with published results. The sample was removed and replaced with the arrangement of quarter-wave plate and horizontal linear polarizer shown in Fig. 5.5. This orientation of the quarter-wave plate converts incident left-handed

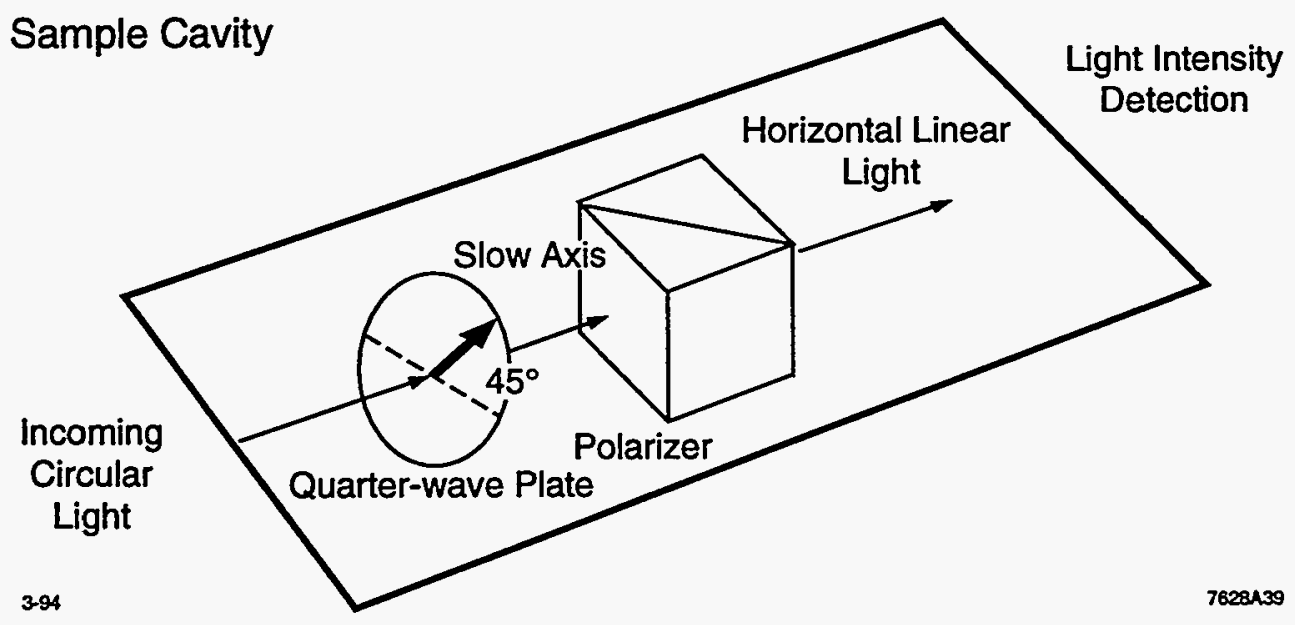

Figure 5.5: Experimental setup for CD-method slow-axis determination.

light into vertical linear light, which is then blocked by the horizontal linear polarizer; thus, this arrangement should yield a measured $\Delta A<0$ in the spectropolarimeter. Since typical CD effects are much smaller than that for the simulated sample in this test, the spectropolarimeter cannot measure the large generated CD; however, the measured sign is correct and unambiguous. These tests again show agreement with the Fresnel method in locating the slow axis of all Compton and PLS quarter-wave plates.

All tests agree in confirming the manufacturer's slow-axis marking of the Compton quarter-wave plates; therefore, the Pockels cell sign determination described earlier can be made. The results of 
these measurements are summarized in Table 5.2 for the two Compton Pockels cells used in the 1992 run. We have found the relationship between the Compton laser bit pattern and the helicity of the

\begin{tabular}{|c|c|}
\hline Compton Pockels Cell ID & Pockels Cell Sign \\
\hline$\# 5$ & -1 \\
\hline$\# 18$ & +1 \\
\hline
\end{tabular}

Table 5.2: Pockels cell sign for Compton Pockels cells used in 1992 run.

light emerging from the Pockels cell. We must also understand the helicity changes in the transport of the photons to the Compton IP. Each mirror reflection in the transport line reverses the photon helicity. The transport line uses only compensated mirror pairs to preserve the magnitude of the light polarization in each $90^{\circ}$ bend; this design ensures also that helicity is unchanged at each bend. It has already been mentioned that the transport line windows have been found free of birefringence in all pressure conditions. To confirm the resulting expectation of helicity preservation at the Compton IP, we use the setup of Fig. 5.1, placing the final quarter-wave plate and horizontal analyzer both immediately after the Compton Pockels cell and at the Compton IP [56]. With positive Pockels cell high voltage, we find that minimum and maximum transmission occur in both locations at the same quarter-wave rotation settings. The test was repeated using negative high voltage with the same result; in this case, however, the intensity extrema were the reverse of those at positive high voltage, indicating the expected opposite-helicity light. Therefore, the photon helicity at the Compton IP is equal to the helicity after the Pockels cell.

As a check on the Fresnel slow-axis technique, a test was made with the PLS and the linac Møller polarimeter [73]. Using the techniques described above, a PLS quarter-wave plate was calibrated and used to find the sign of the PLS Pockels cell. The GaAs source photocathode was then illuminated with positive-helicity light, ejecting positive-helicity electrons. The magnetization field direction in the linac Møller was measured in three independent ways to point along the beam direction, resulting in target electron spins in the opposite direction, due to the negative electron magnetic moment. Since the Møller cross section is larger for the antiparallel spin combination, we expect positive-helicity beam to dominate. This expectation was confirmed in the measured asymmetry, providing independent support for the Pockels cell calibration procedure.

\section{Electron helicity determination}

Knowledge of the theoretical sign of Compton scattering and the pulse-by-pulse light helicity at the Compton IP allows us to determine the electron helicity beam-code assignment for each run by 
simply observing the Compton asymmetry sign for that run. When this is done, we find that the electron helicity beam-code assignment reversed several times during the 1992 run, as described in Table 5.3. All sign-convention reversals are understood in terms of changing spin-rotator conditions.

\begin{tabular}{|c|cc|c|}
\hline SLD runs & \multicolumn{2}{|c|}{ Electron helicity } & Cause of reversal \\
& $\mathbf{1 0}$ & $\mathbf{0 1}$ & \\
\hline $11169-11764$ & - & + & \\
\hline $11764-11874$ & + & - & Accidental RTL reversal \\
\hline $11874-12589$ & - & + & RTL reversal corrected \\
\hline $12589-13962$ & + & - & Intentional LTR reversal \\
\hline
\end{tabular}

Table 5.3: Evolution of electron helicity beam-code assignment throughout 1992 SLD run.

At SLD run 11764, the current in the RTL solenoid was accidentally reversed, rotating the spin in the linac by $180^{\circ}$ relative to the previous running for a given helicity code. This reversal was corrected at SLD run 11874. At run 12589 , the LTR current was intentionally reversed in order to cancel any possible systematic effects due to spin orientation in the damping ring. During the run, the original Compton Pockels cell \#5 became damaged and was replaced with the opposite-sign \#18. This switch in Compton helicity convention is taken into consideration in the assignments of Table 5.3 but of course induces no real electron helicity flip.

By observing Compton-scattering signals directly from the Cerenkov detector, it is possible to check the electron helicity beam-code assignment directly without relying on the integrity of the dataacquisition system. This test was performed late in the run with $S_{P C}=+1$, implying beam codes of 01 and 10 for right- and left-handed laser beam, respectively. The channel 7 Ćerenkov signal was sent directly into an averaging digital oscilloscope, and the electron beam code was maintained at 01 during the test. The observed signal for laser code 01 was clearly larger than that for code 10 , as shown in Fig. 5.6, implying that electron code 01 corresponds to negative-helicity electrons. This assignment agrees with the result for this run period from observation of the recorded measured Compton asymmetry and hence provides a one-time check of sign preservation in the Compton data-acquisition system.

\section{Independent electron helicity test}

A test of electron helicity beam-code assignment, completely independent of the Compton polarimeter, was performed at the end of the run using the extraction-line Møller polarimeter [36]. In order to reduce backgrounds to a tolerable level, the south energy spectrometer magnets were turned off to eliminate beam dispersion and scattering, and the polarimeter detector was brought closer to the 
Electron (01), Photon (01)

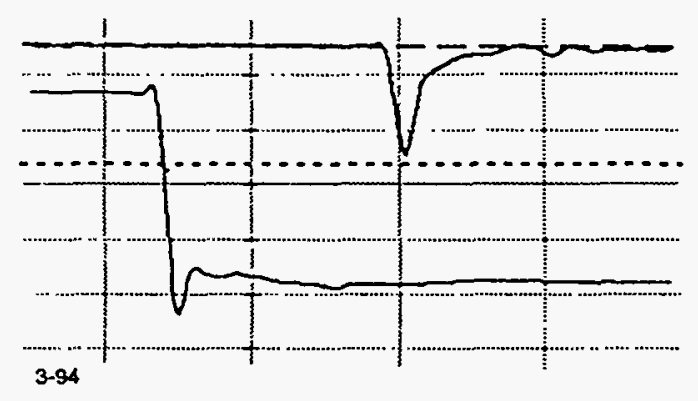

Electron (01), Photon (10)

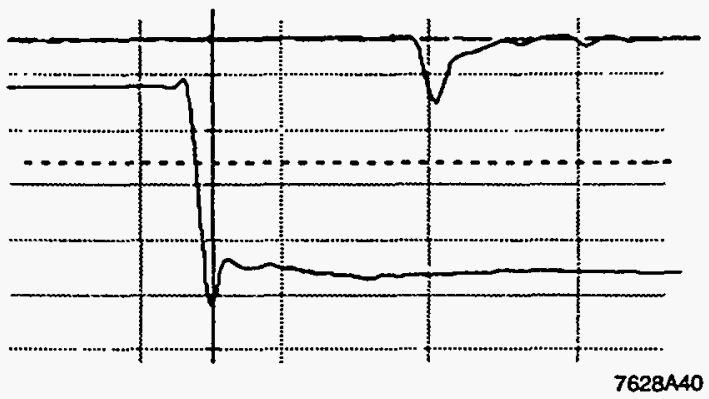

Figure 5.6: Čerenkov chamnel 7 raw signal for 01 electron beam code and 01 (left) and 10 (right) laser beam code.

vacuum exit window, requiring a minor reworking of the beamline geometry. A 2 mil foil, canted at $20^{\circ}$ with respect to the beamline, was selected for the target. ${ }^{2}$ Three independent measurements of the magnetic field at the target found that positive magnet current produced a field pointing along the beamline toward the beam dump, resulting in target electron spin pointing back toward SLD. The magnet current was reversed in each of 75 polarimeter runs taken over eight hours. An experimental asymmetry $A_{E}$ was defined as

$$
A_{E} \equiv \frac{\mathcal{L}_{01} \sigma_{01}-\mathcal{L}_{10} \sigma_{10}}{\mathcal{L}_{01} \sigma_{01}+\mathcal{L}_{10} \sigma_{10}}=\frac{A_{M}+A_{B}}{1+A_{M} A_{B}}
$$

where $\mathcal{L}$ is the beam luminosity, $\sigma$ is the Møller cross section, and the Møller asymmetry $A_{M}$ and luminosity asymmetry $A_{B}$ are defined as

$$
A_{M}=\frac{\sigma_{01}-\sigma_{10}}{\sigma_{01}+\sigma_{10}}
$$

and

$$
A_{B}=\frac{\mathcal{L}_{01}-\mathcal{L}_{10}}{\mathcal{L}_{01}+\mathcal{L}_{10}}
$$

The luminosity asymmetry $\mathcal{L}$ is measured with the beam current toroids; subscripts correspond to electron-pulse helicity codes.

The resulting experimental asymmetries are $A_{E}=(-5.9 \pm 3.0) \times 10^{-4}$ for negative target magnet current (target electron spin pointing toward beam dump), and $A_{E}=(+5.8 \pm 3.3) \times 10^{-4}$ for positive

\footnotetext{
2It is now believed that a miswired target control unit may have inserted a 6 mil transverse target magnetized in the longitudinal direction; this configuration nonetheless yields the same result for the electron helicity.
} 
target magnet current (target electron spin pointing toward SLD). Thus, with our definition of $A_{E}$, and since the antiparallel-spin Møller cross section dominates, beam helicity code 01 corresponds to positive beam helicity at the Møller polarimeter. The $90.5 \mathrm{mr}$ beam deflection angle between the SLD IP and the Møller polarimeter yields a spin rotation of $536.5^{\circ}$ at $45.6 \mathrm{GeV}$, or $3 \pi-3.5^{\circ}$; thus, the electron helicity at the Møller polarimeter is opposite that at the SLD. Therefore, this test finds that the beam helicity code 01 corresponds to negative electron beam helicity at the SLD IP, agreeing with our original results for the bit assignment at the end of the run.

\section{Results of sign check}

In conclusion, all sign checks agree in establishing the electron helicity beam-code assignments of Table 5.3. Using these beam codes, we confirm the original $A_{L R}$ sample breakdown of $5226 \mathrm{Zs}$ made with left-handed electron beam and $4998 Z \mathrm{~s}$ made with right-handed beam. Therefore, the measured value of $A_{L R}$ is positive, in agreement with the Standard Model prediction.

\subsubsection{Total systematic uncertainty}

Table 5.4 summarizes all non-negligible systematic uncertainties in the measurement of $A_{L R}$. The polarization information of Table 3.4 is included, as well as the secondary systematics discussed above.

\begin{tabular}{|c|c|c|}
\hline Systematic uncertainty & $\delta \mathcal{P} / \mathcal{P}$ & $\boldsymbol{6 A}_{\mathbf{L R}} / \boldsymbol{A}_{\mathbf{L R}}$ \\
\hline Laser polarization & $2.0 \%$ & \\
Detector linearity & $1.5 \%$ & \\
Interchannel consistency & $0.9 \%$ & \\
Spectrometer calibration & $0.4 \%$ & \\
Electronic noise correction & $0.4 \%$ & \\
\hline Total polarization uncertainty & $2.7 \%$ & $2.7 \%$ \\
Luminosity asymmetry & & $1.9 \%$ \\
Background fraction & & $0.7 \%$ \\
\hline Total systematic uncertainty & & $\mathbf{3 . 4 \%}$ \\
\hline
\end{tabular}

Table 5.4: Contributions to the systematic error in the measurement of $A_{L R}$.

\subsection{A $A_{L R}$ Result}

Having completed the event selection and evaluated all experimental systematic errors, we can now calculate the measured value of $A_{L R}$ and its associated uncertainty. In addition, we translate this 
value into a measurement of the weak mixing angle and compare our results with those of other experiments and the Standard Model.

\section{Calculation of $A_{L R}$ and $\sin ^{2} \theta \underset{W}{\mathrm{eff}}$}

We use Eq. 5.1 to find the experimental result for $A_{L R}$. With the values of $\overline{\mathcal{P}}$ from Eq. 3.14 and $A_{m}$ from Eq. 4.1, along with the secondary systematics discussed earlier this chapter, we find the result for $A_{L R}$ :

$$
A_{L R}=0.1009 \pm 0.0442 \text { (stat.) } \pm 0.0035 \text { (syst.) }
$$

We see that the measurement error is completely dominated by the statistical uncertainty. To convert $A_{L R}$ into a measurement of $\sin ^{2} \theta_{W}^{\text {eff }}\left(M_{Z}^{2}\right)$, we first correct the measurement for the effects of initial-state radiation and off-peak running, and then use Eq. 1.35 to extract $\sin ^{2} \theta_{W}^{\mathrm{eff}}\left(M_{Z}^{2}\right)$. The correction is made using the information in Fig. 1.9: we find that initial-state radiation shifts $A_{L R}$ by -0.0045 and running at the average center-of-mass energy of $91.55 \mathrm{GeV}$ shifts $A_{L R}$ by +0.0069 from the value at the $Z$-peak energy of $91.187 \mathrm{GeV}$, where $A_{L R}$ is defined. Therefore, we must correct our measurement of $A_{L R}$ down by 0.0024 in order to extract $\sin ^{2} \theta_{W}^{\text {eff }}\left(M_{Z}^{2}\right)$ [74]. Eq. 1.35 then yields

$$
\sin ^{2} \theta_{W}^{\mathrm{eff}}\left(M_{Z}^{2}\right)=0.2377 \pm 0.0056 \text { (stat.) } \pm 0.0005 \text { (syst.) }
$$

A comparison of this measurement of $A_{L R}$ with recent $\tau$-polarization results from LEP [75] is shown in Fig. 5.7. Within the Standard Model and given lepton universality, the average $\tau$-polarization equals $A_{L R}$ and can be directly compared. All results agree with each other and are consistent with Standard-Model predictions for allowed top and Higgs masses.

Comparing our $A_{L R}$ and $\sin ^{2} \theta_{W}^{\text {eff }}\left(M_{Z}^{2}\right)$ results with Figs. 1.10 and 1.7, we find that the large uncertainty allows 1- $\sigma$ Standard-Model agreement for top masses up to $150 \mathrm{GeV}$ at the lowest allowed Higgs mass of $\sim 50 \mathrm{GeV}$ and agreement for top masses up to $200 \mathrm{GeV}$ with the largest allowable Higgs mass of $\sim 2 \mathrm{TeV}$. At two standard deviations, all conceivable experimentally and theoretically allowed mass combinations of $50<m_{t}<250 \mathrm{GeV}$ and $50<m_{H}<2000 \mathrm{GeV}$ are allowed. Therefore, a more precise measurement of $A_{L R}$ is necessary to begin to constrain the unknown masses and to compare the allowed ranges with other experimental results. 


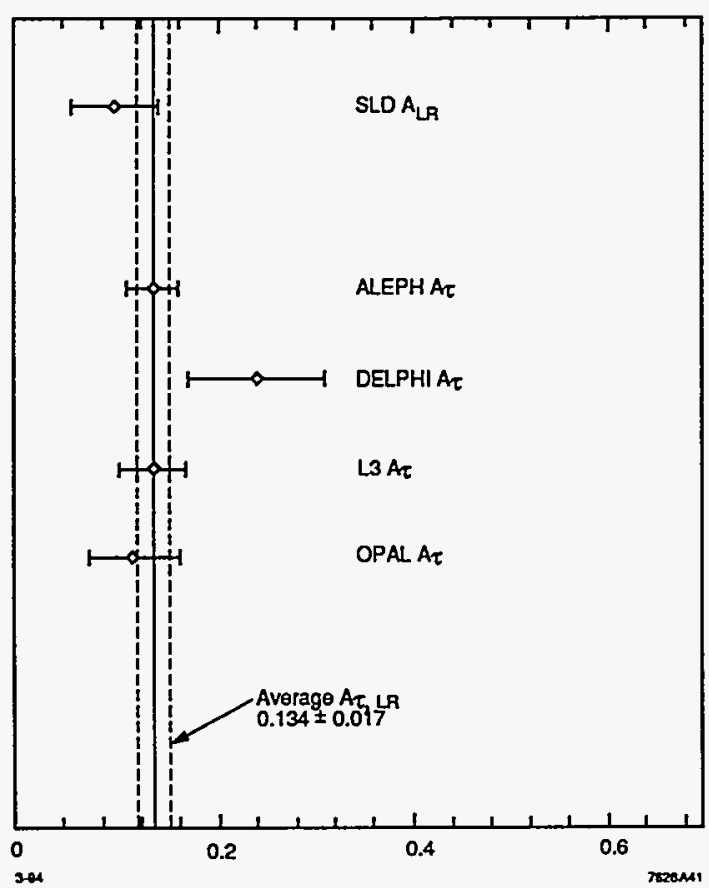

Figure 5.7: 1992 SLD $A_{L R}$ measurement compared with recent average $\tau$-polarization measurements from the four LEP experiments.

\subsection{Future Prospects}

The SLC/SLD program continues to measure the left-right asymmetry with increasing luminosity, polarization, and systematic precision. In the 1993 run, use of a strained lattice cathode resulted in an average beam polarization of $>60 \%$; coupled with the improved luminosity yielding $\sim 5 \times 10^{4}$ $Z$ events, the uncertainty in $\sin ^{2} \theta_{W}$ was reduced to $\sim 0.0009$. Future SLC/SLD running may provide a total of $5 \times 10^{5}$ to $10^{6} Z \mathrm{~s}$ at high polarization and $\sim 1 \%$ relative polarization uncertainty. The precision in the $\sin ^{2} \theta_{W}$ measurement as a function of $Z$ count, beam polarization, and polarization measurement error is shown in Fig. 5.8. Predictions for future LEP measurements are indicated in the figure. Using the LEP results from 1992 [76] and the projected doubling of the sample size, and assuming conservatively that the current $\tau$-polarization measurements are completely statistically limited, we arrive at an ultimate precision of $\sim 0.0021$ for the ALEPH $\tau$-polarization determination of $\sin ^{2} \theta_{W}$ and $\sim 0.0013$ for the combined LEP $\sin ^{2} \theta_{W}$ value. This latter precision is achieved at SLC/SLD with $\sim 3 \times 10^{4} Z$ s at $60 \%$ polarization. We can estimate the ultimate precision attainable at LEP using all asymmetry information with the final sample size. Using the current all-LEP, all-asymmetry precision of $\delta \sin ^{2} \theta_{W} \sim 0.0006$ [76] and assuming partial statistical limitation and a 


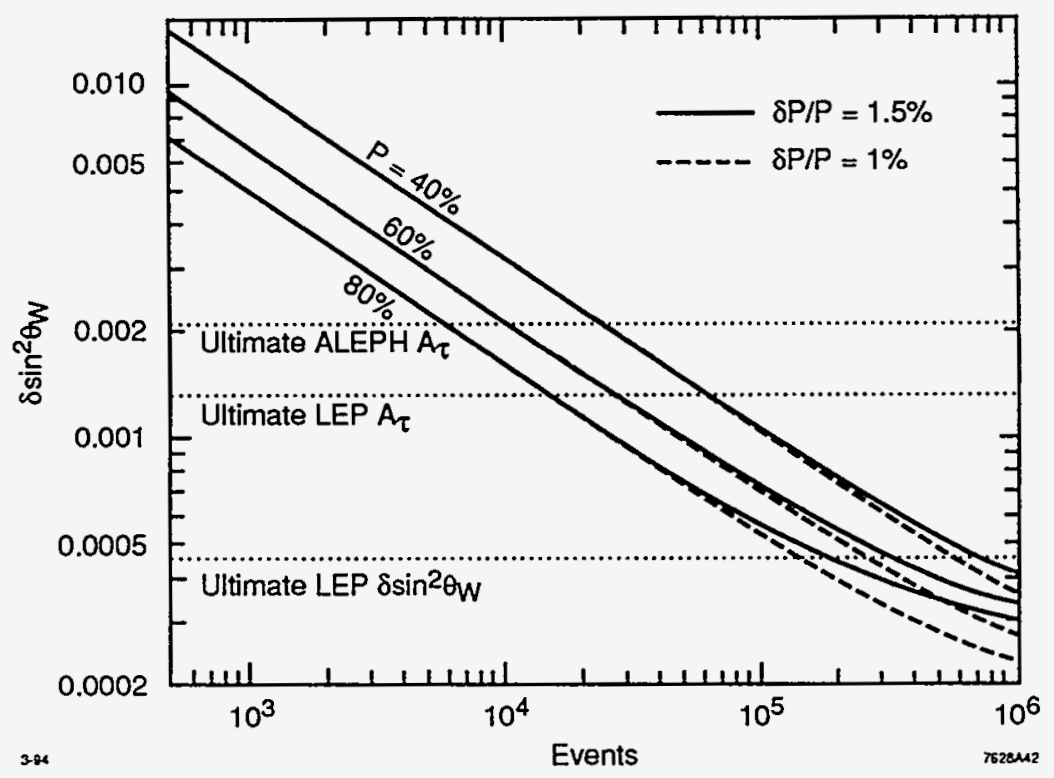

Figure 5.8: Precision in the measurement of $\sin ^{2} \theta_{W}$ as a function of $Z$ count, polarization, and polarization-measurement precision. Projected future LEP measurements are shown.

doubling of the current sample size, we arrive at a possible $\delta \sin ^{2} \theta_{W}$ of $\sim 0.00045$. This precision is attainable with $A_{L R}$ at a sample size of $<2 \times 10^{5} Z$ s and $70 \%$ polarization, assuming systematic precision of $\sim 1 \%$ can be achieved. These experimental goals are expected to be achieved in the next year or two of SLC/SLD running. This measurement of a single quantity by a single experiment of comparable precision to that of the convolution of many tens of competing measurements will be an outstanding and critically important contribution to the study of electroweak interactions. 


\section{Bibliography}

[1] The experimental results presented in this thesis have been published in K. Abe et al., Phys. Rev. Lett. 70, 2515 (1993).

[2] S. L. Glashow, Nucl. Phys. 22, 579 (1961); S. Weinberg, Phys. Rev. Lett. 19, 1264 (1967); A. Salam and J. C. Ward, Phys. Lett. 13, 168 (1964).

[3] We follow the notation and exposition of F. Halzen and A. D. Martin, Quarks and Leptons: An Introductory Couse in Modern Particle Physics, John Wiley \& Sons, New York, 1984.

[4] $\alpha$ : E. R. Cohen and B. N. Taylor, Rev. Mod. Phys. 59, 1121 (1987). $G_{F}$ : K. Hikasa et al., Phys. Rev. D45, Part II (1992). $M_{Z}$ : CERN-PPE/93-53, March 1993.

[5] A review of renormalization of weak interaction parameters is given in M. E. Peskin, Theory of Precision Electroweak Measurements, SLAC-PUB-5210, March 1990.

[6] R. Cahn, Phys. Rev. D36, 2666 (1987), and [5] above; the original expression for first-order radiative corrections to a narrow resonance is found in G. Bonneau and F. Martin, Nucl. Phys. B27, 381 (1971).

[7] E. A. Kuraev and V. S. Fadin, Sov. J. Nucl. Phys. 41, 466 (1985).

[8] A. Sirlin, Nucl. Phys. B71, 29 (1974); Nucl. Phys. B100, 291 (1975).

[9] M. Consoli and W. Hollik, Z Physics at LEP 1, Proceedings of the Workshop, Geneva, Switzerland, 1989, edited by G. Altarelli, R. Kleiss, and C. Verzegnassi (CERN Report No. CERN89-08, Geneva, 1989), Vol. 1.

[10] See, e.g., W. F. L. Hollik, Fort. Phys. 38, 165 (1990); D. Kennedy and B. W. Lynn, Nucl. Phys. B322, 1 (1989). 
[11] ZSHAPE: W. Beenakker and W. Hollik, Ref. [9], Vol. 3. EXPOSTAR: D. C. Kennedy et al., SLAC-PUB-4128 (1988). ZFITTER: D. Bardin et al., Comput. Phys. Commun. 59, 303 (1990); Z. Phys. C44, 493 (1989); Nucl. Phys. B351, 1 (1991); Phys. Lett. B229, 405 (1989).

[12] The program ZSHAPALR calculates cross sections, including initial-state radiation, separately for left- and right-handed electrons (J. Yamartino, Modifications to ZSHAPE, SLD Electroweak Physics Working Group note, December 1992).

[13] M. E. Levi et al., Phys. Rev. Lett. 51, 1941 (1985).

[14] W. Bartel et al., Phys. Lett. B99, 281 (1981).

[15] B. W. Lynn and C. Verzegnassi, Phys. Rev. D35, 3326 (1987).

[16] A discussion of precision electroweak tests and a parametrization scheme describing extrastandard-model effects is given in M. E. Peskin and T. Takeuchi, Phys. Rev. D46, 381 (1992).

[17] M. Swartz, Calculating $A_{L R}$ from Our Data (Updated), SLD Electroweak Physics Working Group note, June 1992.

[18] D. Blockus et al., Proposal for Polarization at the SLC, SLAC-PROPOSAL-SLC-UPGRADE01, April 1986.

[19] N. Phinney, SLAC-PUB-5864, August 1992.

[20] SLAC Linear Collider Conceptual Design Report, SLAC Report SLAC-229, June 1980.

[21] D. T. Pierce and F. Meier, Phys. Rev. B13, 5484 (1976).

[22] C. Y. Prescott et al., Phys. Lett. B77, 347 (1978).

[23] T. Junk and T. Johnson, SLD Note \#227, November 1992.

[24] T. Maruyama et al., SLAC-PUB-6033, January 1993.

[25] D. Schultz et al., SLAC-PUB-5768, March 1992.

[26] M. Woods et al., SLAC-PUB-5984, September 1992.

[27] T. Maruyama and R. Prepost, private communication.

[28] L. T. Thomas, Phil. Mag. 3, 1 (1927). 
[29] T. Fieguth and M. Petradza, private communication.

[30] A. A. Sokolov and I. M. Ternov, Soviet Physics-Doklady 8, 1203 (1964).

[31] V. N. Baier and V. M. Katkov, Phys. Lett. 27A, 327 (1967), and Soviet Physics-JETP 25, 944 (1967).

[32] R. H. Helm and W. P. Lysenko, SLAC-TN-72-1, March 1972 and M. J. Alguard et al., Nucl. Inst. and Meth. 163, 29 (1979).

[33] K. Yokoya and P. Chen, SLAC-PUB-4692, September 1988.

[34] J. Kent et al., SLAC-PUB-4922, March 1989.

[35] P. Rowson, private communication.

[36] R. King and M. Swartz, SLD Note \#225, September 1992.

[37] P. C. Rowson et al., SLD Note \#216 (revised), October 1992.

[38] M. Swartz, private communication.

[39] The SLD Design Report, SLAC Report 273, May 1984.

[40] C. J. S. Damerell et al., Nucl. Inst. and Meth. A253, 478 (1987).

[41] D. Axen et al., Nucl. Inst. and Meth. A328, 472 (1993).

[42] LAC energy resolution and calibration of the LAC energy scale is discussed in S. González, SLD Physics Note \#24, October 1993.

[43] A. C. Benvenuti et al., Nucl. Inst. and Meth. A289, 463 (1990).

[44] S. C. Berridge et al., IEEE Trans. Nucl. Sci. NS-36, 339 (1989); S. C. Berridge et al., IEEE Trans. Nucl. Sci. NS-37, 1191 (1990); S. C. Berridge et al., IEEE Trans. Nucl. Sci. NS-39, 1242 (1992).

[45] H. Frauenfelder and A. Rossi, Vol. 5, Part B, Methods of Experimental Physics, edited by I. C. L. Yuan and C. S. Wu, Academic Press, New York, 1963.

[46] C. Y. Prescott, SLAC-TN-73-1, January 1973.

[47] Morris L. Swartz, SLAC-PUB-4656, June 1988. 
[48] H. Veltman, LBL-27163, May 1989.

[49] W. R. Nelson, H. Hirayama, D. W. O. Rogers, SLAC-Report-265, December 1985.

[50] All beam parameters: N. Toge, private communication.

[51] H. E. Watson and K. L. Ramaswamy, Proc. of the Royal Soc. of London 156, 144 (1936).

[52] J. T. Gary and L. W. Pickett, J. Chem. Phys. 22, 599 (1954).

[53] W. F. Kieffer and J. P. Howe, J. Am. Chem. Soc. 64, 1 (1942).

[54] The polarization determination is summarized in B. Schumm and R. Elia, SLD Note \#222, November 1992.

[55] Calculated by M. Swartz from the south arc TRANSPORT deck, private communication.

[56] M. Fero et al., SLD Note \#221, October 1992.

[57] M. Fero et al., SLD Note \#212 (revised), August 1992.

[58] A. Lath, private communication.

[59] H. Park et al., SLD Physics Note \#17, March 1993.

[60] R. Ben-David and H. Park, SLD Physics Note \#18, July 1993.

[61] P. N. Burrows et al., SLD Note \#229, January 1993.

[62] DELPHI collaboration: P. Abreu et al., Phys. Lett. B268, 296 (1991); L3 collaboration: O. Adriani et al., Phys. Lett. B288, 404 (1992); OPAL collaboration: M. Akrawy et al., Phys. Lett. B257, 531 (1991).

[63] E. Vella and J. Yamartino, SLD Note \#213, February 1992.

[64] A. Bazarko, SLD internal memo.

[65] J. M. Yamartino, SLD Physics Note \#14, November 1992.

[66] T. Himel, private communication.

[67] G. Bonvicini et al., Phys. Rev. Lett. 62, 2381 (1989).

[68] P. C. Rowson et al., SLD Note \#219, September 1992. 
[69] A. Lath and M. Fero, SLD Note \#220, October 1992.

[70] S. D. Drell, SLD Note \#226, September 1992.

[71] A detailed derivation is given in M. Woods, SLD Note \#223, October 1992.

[72] S. Jacobs et al., J. Opt. Soc. Am. B5 (1988).

[73] H. Band, R. Prepost, M. Woods, SLD Note \#224, January 1993.

[74] This result of the ZSHAPE program has been verified with EXPOSTAR.

[75] ALEPH collaboration: D. Decamp et al., Z. Phys. C53, 1 (1991) and Phys. Lett. B265, 430 (1991); DELPHI collaboration: P. Aarnio et al., Nucl. Phys. B367, 511 (1992) and P. Abreu et al., Z. Phys. C55, 555 (1992); L3 collaboration: B. Adeva et al., Z. Phys. C51, 179 (1991) and O. Adriani et al., Phys. Lett. B294, 466 (1992); OPAL collaboration: G. Alexander et al., Z. Phys. C52, 175 (1991) and G. Alexander et al., Phys. Lett. B266, 201 (1991).

[76] Unpublished; results from M. Mannelli, lecture at $21^{\text {st }}$ SLAC Summer Institute, Spin Structure in High Energy Physics, Stanford, CA, August 1993. 